UNIVERSIDADE DE BRASÍLIA

FACULDADE DE TECNOLOGIA

DEPARTAMENTO DE ENGENHARIA MECÂNICA

NOVA FORMULAÇÃO DO MÉTODO DOS ELEMENTOS DE CONTORNO PARA ASSOCIAÇÃO DE PLACAS ESPESSAS

DALMO INÁCIO GALDEZ COSTA

ORIENTADOR: ÉDER LIMA DE ALBUQUERQUE

DOUTORADO EM CIÊNCIAS MECÂNICAS

PUBLICAÇÃO: ENM.TD - 27/14

BRASÍLIA/DF: NOVEMBRO-2014 



\author{
UNIVERSIDADE DE BRASÍLIA \\ FACULDADE DE TECNOLOGIA \\ DEPARTAMENTO DE ENGENHARIA MECÂNICA
}

\title{
NOVA FORMULAÇÃO DO MÉTODO DOS ELEMENTOS DE CONTORNO PARA ASSOCIAÇÃO DE PLACAS ESPESSAS
}

\section{DALMO INÁCIO GALDEZ COSTA}

Relatório submetido como requisito parcial para obtenção do grau de Doutor em Ciências Mecânicas pelo Departamento de Engenharia Mecânica da Universidade de Brasília.

Banca Examinadora:

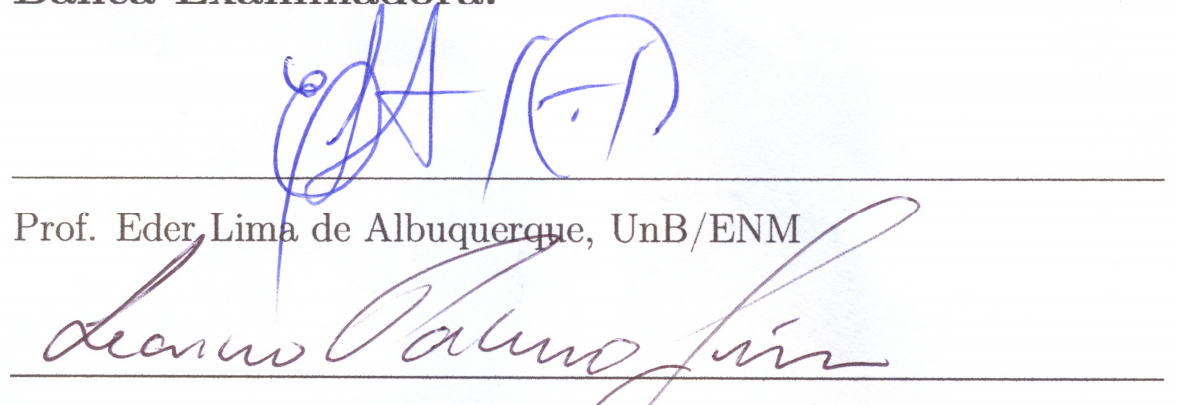

Prof. Leandro Palermo Júnior, Unicamp/FEC

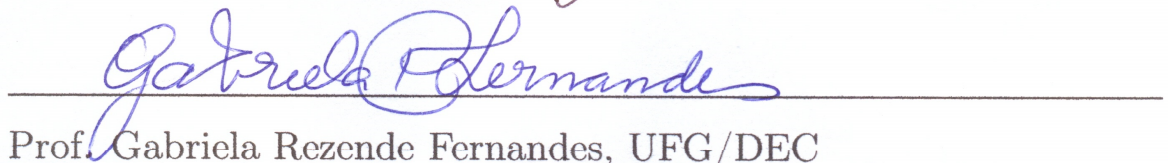

Prof Gabriela Rezende Fernandes, UFG/DEC

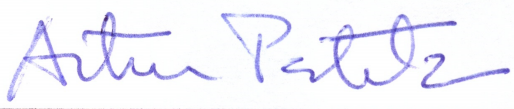

Prof. Artur António de Almeida Portela, UnB/ENC

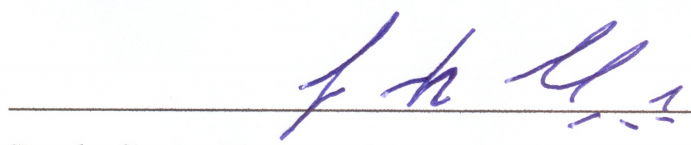

Prof. Carla Tatiana Mota Anflor, UnB/FGA 
Ficha catalográfica elaborada pela Biblioteca Central da Universidade de Brasília. Acervo 1019238.

Costa, Dalmo Inácio Galdez.

C837n Nova formulação do método dos elementos de contorno para associação de placas espessas / Dalmo Inácio Galdez Costa. - 2014.

xix, $113 \mathrm{f} .:$ il. ; $30 \mathrm{~cm}$.

Tese (doutorado) - Universidade de Brasília, Faculdade de Tecnologia, Departamento de Engenharia Mecânica,

Pós-Graduação em Ciências Mecânicas, 2014.

Orientação: Éder Lima de Albuquerque.

Inclui bibliografia.

1. Métodos de elementos de contorno. 2. Elasticidade. 3. Placas (Engenharia). 4. Cisalhamento. I. Albuquerque, Éder Lima de. II. Título. 


\section{Dedicatória}

Dedico este trabalho a todos aqueles que fizeram parte, ajudando a desenvolvê-lo ou mesmo na convivência durante esta longa jornada. 


\section{Agradecimentos}

- À minha mãe, Rosenilde, pelo amor e ajuda em todos os momentos.

- Ao meu pai e irmãos, Domingos, Diego e Anara, pelo apoio ao longo desta longa caminhada.

- Ao meu amigo Pablo Mesquita, que injustamente foi esquecido nos agradecimentos da dissertação, pelo companheirismo e amizade mesmo após tanto tempo distante.

- À minha namorada Thâmara, pelo amor, ajuda, apoio, incentivo e compreensão ao longo desta caminhada.

- Ao meu orientador, Eder Lima de Albuquerque, pela dedicação em ensinar, cuidado durante todos esses anos de trabalho e pela imensa quantidade de conhecimento transmitido.

- Ao Professor Pedro Miguel Baiz Villafranca pela proposição do tema, coorientação e frutíferas discussões em relação ao trabalho.

- Aos amigos que fiz durante o mestrado: Adilto Cunha, Adriana dos Reis, Audirene Amorim, Carlos Magno, Carolina Matsuo, Denize Oliveira, Doval, Eveline Soares, Fabiano, Fábio Dalmazzo, Gisele Gonçalves, Giselle Barata, Hairton, Karlos Braga, Kerlles Rafael, Lourival Matos e Patrícia, Louryval Paixão, Luís Jorge, Núbia, Raquel, René Quispe e Solange que tornaram o período em Campinas muito mais agradável.

- Aos amigos que fiz durante o doutorado: Túlio Humberto, Túlio Avelar, Patrícia Dantas, Marcus Sá, Volker Francestein, Miguel Garcia, Sílvio, Jesus Maurício González, Paula Duque, Vagner, Alexander, Braitner Lobato, Miélle Pestana, Guilherme Ferreira, Jhonny, Carolina Burbano, Nuno Dias, Bráulio Gutierrez, Adriano Possebon, Karen Fabara, Tatiane Leal, Thiago, Adrian Bentacur, Jonatas Cunha, Felipe Dias, Alessandra Nakazato, Taíse Ferreira, Rafaell Reboredo, Gustavo Gontijo, Lucas Campos, Cybelle, Frederico Vilela, Thamise Vilela, Luciana e Jefferson Cardoso. Pelos estudos em conjunto, companheirismo e pelos momentos de descontração.

- Ao amigo André pelo incentivo, ajuda e companheirismo durante essa jornada do doutorado.

- Aos amigos do IFMA, Professor Keyll Carlos, Luciano, Rita de Cássia, André, Gricirene, Izaque, Danúbia e muitos outros pela amizade e pelo apoio nos tempos da graduação.

- Aos professores que me ajudaram ou orientaram em algum momento, Flamínio Levy (UnB), Renato Pavanello (Unicamp), Rogério Markzac (UFRGS), 
Itamar Ferreira (Unicamp), Paulo Sollero (Unicamp), Carla Anflor (UnB), Edgar Mamiya (UnB), Lucival Malcher (UnB), Aline de Paula (UnB), Luciano Mendes (UnB), Artur Portela (UnB), Carlos Cimini (UFMG), Carlos Daros (Unicamp), Taygoara Felamingo (UnB), Marcus Girão (UnB), Denise Santos (Cultura Inglesa).

- À FAP-DF e à CNPq pelo apoio financeiro que permitiu a apresentação de trabalhos relacionados a esta tese em vários congressos internacionais.

- À Capes pelo apoio financeiro durante o desenvolvimento deste trabalho. 
1 Introdução 1

1.1 Revisão Bibliográfica . . . . . . . . . . . . . . . . . . . 3

1.2 Divisão do trabalho . . . . . . . . . . . . . . . . . . . . . . . . 7

2 Teoria da Elasticidade $\quad 8$

2.1 Introdução . . . . . . . . . . . . . . . . . . . . . . . . . 8

2.2 Elasticidade . . . . . . . . . . . . . . . . . . . . . 8

2.2.1 Relação entre deformações e deslocamentos . . . . . . . . . . . . 9

2.2.2 Compatibilidade de deformações . . . . . . . . . . . . . . . 11

2.2.3 Tensões e equilíbrio . . . . . . . . . . . . . . . . . . 12

2.2.4 Comportamento do material em regime elástico . . . . . . . . . 15

2.2.5 Condição de Tensão Plana . . . . . . . . . . . . . . . . . . . . 17

2.3 Teoria de placas deformáveis por cisalhamento . . . . . . . . . . . . 17

2.3.1 Equações de Equilíbrio . . . . . . . . . . . . . . . . . . . 18

2.3.2 Relações tensões resultantes - deformações . . . . . . . . . . . . 19

2.3.3 Tipos de condições de contorno . . . . . . . . . . . . . 23

3 Método dos Elementos de Contorno $\quad 24$

3.1 Formulação do MEC para Elasticidade . . . . . . . . . . . . . . . . . . 24

3.1 .1 Equação integral de contorno para elasticidade . . . . . . . . . 26

3.1 .2 Soluções Fundamentais . . . . . . . . . . . . . . . . . . 28

3.1.3 Equação integral de contorno de elasticidade especificada para pontos no contorno . . . . . . . . . . . . . 30

3.2 Formulação do MEC para Placas Espessas . . . . . . . . . . . . . . . . 31

3.2.1 Equação integral de contorno para placas espessas . . . . . . . . 32

3.2 .2 Soluções fundamentais . . . . . . . . . . . . . . . . . . . . 33

3.2.3 Equação integral de contorno de placas especificada para pontos no contorno . . . . . . . . . . . . . . . . 34

3.3 Discretização do problema e Método dos Elementos de Contorno . . . . 35

3.3 .1 Funções de forma . . . . . . . . . . . . . . . . 36

3.3.2 Integração dos elementos . . . . . . . . . . . . . . . . . . 41

3.3 .3 Integração Numérica . . . . . . . . . . . . . . . . . . . . . . . 43

3.4 Tratamento de singularidades . . . . . . . . . . . . . . . 45

3.4.1 Técnica de subdivisão de elementos . . . . . . . . . . . . . . . 45

3.4 .2 Técnica de transformação de variáveis . . . . . . . . . . . . . 46 
3.4.3 Considerações de corpo rígido generalizados . . . . . . . . . . 47

4 Formulações do MEC para drilling rotation $\quad 48$

4.1 Introdução . . . . . . . . . . . . . . . . . . . . 48

4.2 PUBEM e XBEM . . . . . . . . . . . . . . . . . . . . . . . . 48

4.2.1 Relacão entre as rotações e a rotação da teoria da elasticidade. . 54

4.2.2 Equações integrais com elementos de contorno . . . . . . . . . 56

4.3 Equação integral de contorno para rotação no plano . . . . . . . . . . . 56

4.3.1 Equação integral de contorno para rotação no plano com elementos de contorno . . . . . . . . . . . . . . . . 57

4.4 Tratamento de singularidades . . . . . . . . . . . . . 59

5 Formulação Acoplada para associação de Placas e aspectos computacionais $\quad 62$

5.1 Introdução . . . . . . . . . . . . . . . . . . . . . . 62

5.2 Associação de Placas Espessas . . . . . . . . . . . . . . . . . 62

5.3 MEC com sub regiões . . . . . . . . . . . . . . . . . . . . . . . . . . 64

5.4 Aspectos computacionais . . . . . . . . . . . . . 67

6 Resultados numéricos para Elasticidade Plana $\quad 69$

6.1 Introdução . . . . . . . . . . . . . . . . . . . . . . . 69

6.2 Solução analítica bidimensional para uma viga . . . . . . . . . . . . . . 69

6.3 Viga em balanço com baixa razão de aspecto . . . . . . . . . . . . . 70

6.3.1 Resultados para condições de contorno corrigidas . . . . . . . . 74

6.4 Membrana de Cook . . . . . . . . . . . . . . . . . . . . . 77

6.5 Viga em balanço com alta razão de aspecto . . . . . . . . . . . . . . 80

6.5.1 Viga com alta razão de aspecto com sub regiões . . . . . . . . . 84

6.5.2 Viga com alta razão de aspecto com aumento no número de pontos de integração . . . . . . . . . . . . . . 86

7 Resultados Numéricos para associação de placas $\quad 88$

7.1 Introdução . . . . . . . . . . . . . . . . . . . . 88

7.2 Associação em L . . . . . . . . . . . . . . . . . . . . . . . . . . . . . . . . . 88

7.3 Associação em L em ângulos não-retos . . . . . . . . . . . . . . . . . 90

7.4 Viga em formato de caixa . . . . . . . . . . . . . . . . . 95

7.4.1 Resultados usando elementos quadráticos . . . . . . . . . . . . 102

7.5 Associação em L com carregamento lateral . . . . . . . . . . . . . . . . 104 
8 Considerações Finais $\quad 107$

8.1 Conclusões . . . . . . . . . . . . . . . . . . . . . . 107

8.2 Trabalhos Futuros . . . . . . . . . . . . . . . . . . . . 108 


\section{RESUMO}

Nesta tese a formulação do método dos elementos de contorno é desenvolvida para a análise de estruturas formadas pela associação tridimensional de placas espessas. Esta abordagem apresenta seis graus de liberdade por nó, três deslocamentos e três rotações. É construída a partir da associação das formulações de elasticidade plana e placas deformáveis por cisalhamento. A partir destas, dois deslocamentos vem da formulação plana, um deslocamento e duas rotações da formulação de placas. Para que se obtenha a terceira rotação, uma equação da drilling rotation é incluída aplicando a equação integral de contorno na elasticidade plana na expressão analítica da rotação, oriunda da teoria da elasticidade. No arranjo tridimensional, cada placa é definida como uma sub região e os termos locais são calculados. Após a necessária transformação dessas equações para um plano de referência comum, as condições de compatibilidade de deslocamentos e rotações, e equilíbrio de momentos e forças de superfície são impostas. Vários testes numéricos são utilizados para validação de ambos, problemas de elasticidade plana e para a formulação de associação de placas. Finalmente, estes resultados são comparados com soluções analíticas e aqueles obtidos com o método dos elementos finitos.

Palavras Chave: Elasticidade Plana, Drilling rotation, Método dos elementos de contorno, Associação de placas deformáveis por cisalhamento. 


\begin{abstract}
In this thesis a boundary element formulation is developed for the analysis of structures formed by three-dimensional association of plates. A plate boundary element formulation is developed with six degrees of freedom per node given by three displacements and three rotations. It is built from the association of plane elasticity and shear deformable plate formulations. From these formulations, we have two displacements from plane elasticity, one displacement and two rotations from the shear deformable plate formulation. In order to obtain three rotations, an equation for the out of plane rotation (drilling rotation) is included applying plane elasticity boundary integral equations in the analytical expression for rotation. In the three-dimensional assembly, each plate element is defined as a sub-domain for the use of multi regions technique. After the necessary transformation of these equations to a common reference system, displacement-rotation compatibilities and traction-moment equilibrium conditions are taken into account. A number of numerical tests is presented for both, plane elasticity and shear deformable plate association problems. These results are compared to analytical solutions and finite element results.
\end{abstract}

Keywords: Plane Elasticity, Drilling rotation, Boundary Element Method, Folded shear deformable plates. 


\section{Lista de Figuras}

1.1 Carro de automobilismo da categoria Fórmula 1, exemplo de projeto com utilização das técnicas e materiais mais avançados. . . . . . . . . .

2.1 Exemplo de barra sofrendo uma deformação sobre a ação de um carregamento axial. . . . . . . . . . . . . . . . . .

2.2 Deslocamento de um ponto dentro de um corpo elástico sólido, inicialmente na configuração não deformada em linha cheia e na deformada em linha tracejada $[1] \ldots \ldots$. . . . . . . . . . . . . . . .

2.3 Representação de um sólido genérico sob a ação de carregamentos arbitrários. . . . . . . . . . . . . . . . . . . . . 13

2.4 Planos coordenados com respectivos valores de tensão. . . . . . . . . . 13

2.5 Plano oblíquo mostrando normal e uma resultante de força de superfície. 14

2.6 Situação de equilíbrio para um elemento de placa. Os carregamentos foram separados em duas imagens para facilitar a visualização. . . . . . 18

2.7 Ilustração mostrando as variáveis do problema de placa espessa em um ponto do contorno [2].

3.1 Ponto fonte e campo nos quais estão localizados uma carga pontual unitária e as deslocamento correspondente. . . . . . . . . . . . . . . . . 29

3.2 Discretização de um problema em elementos de contorno. . . . . . . . . 35

3.3 Representação de quatro tipos de elementos, a) lineares contínuos, b) lineares descontínuos, c) quadráticos contínuos, d) quadráticos descon-

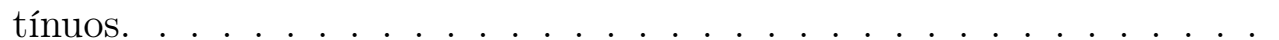

4.1 Representação esquemática da coordenada paramétrica $\xi$ no plano $x_{1}-$ $x_{2}$ e do sistema local $n-t$ para o caso de um elemento linear contínuo. $\quad 50$

4.2 Relação entre derivadas no sistema local e global. . . . . . . . . . . . . 52

5.1 Representação esquemática de duas placas coplanares. . . . . . . . . . . 64

5.2 Associação de placas mostrando os sistemas de coordenada global e local. 65

5.3 Exemplo de visualização de geometria e condições de contorno. . . . . . 68

6.1 Ilustração da viga em balanço. A carga cisalhante distribuída na ponta

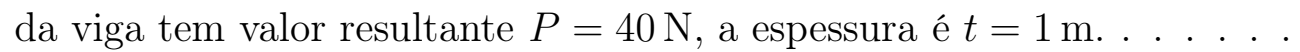

6.2 Exemplo de um modelo discretizado em elementos de contorno lineares descontínuos para a viga curta. Neste caso foram utilizados 20 elementos. 71

6.3 Análise de convergência do deslocamento $u_{2}$ no ponto A para a viga curta. 71 
6.4 Análise de convergência da rotação $\omega_{3}$ no ponto A para a viga curta. $\quad$. 72

6.5 Erro calculado para $u_{2}$ em função do número de nós para viga curta. 73

6.6 Erro calculado para $\omega_{3}$ em função do número de nós para viga curta. 73

6.7 Modelo da viga com condições de contorno corrigidas. A resultante de carga na extremidade da viga é igual $P=40 N$ e possui distribuição parabólica. Os deslocamentos $u_{1}$ (vermelho) e $u_{2}$ (azul) variam de acordo com a solução analítica. . . . . . . . . . . . . . . . . .

6.8 Resultados de $u_{2}$ no ponto A para a viga curta com condições de contorno corrigidas. . . . . . . . . . . . . . . . . . .

6.9 Resultados de $\omega_{3}$ no ponto A para a viga curta com condições de contorno corrigidas. . . . . . . . . . . . . . . . . . .

6.10 Erros relativos para $u_{2}$ em função do número de pontos de colocação em escala logarítmica com condições de contorno corrigidas. . . . . . . . .

6.11 Erros relativos para $\omega_{3}$ em função do número de pontos de colocação em escala logarítmica com condições de contorno corrigidas.

6.12 Modelo da membrana de Cook. As propriedades mecânicas são: $E=$ $1 \mathrm{~Pa}, \nu=1 / 3$. O carregamento distribuído tem resultante $P=1 \mathrm{~N}$ e espessura $t=1 \mathrm{~m} \ldots \ldots \ldots \ldots \ldots \ldots \ldots$

6.13 Exemplo de malha para a membrana de Cook com 40 elementos lineares descontínuos.

6.14 Distribuição de deslocamentos totais para a membrana de Cook. . . . . 78

6.15 Resultados de deslocamentos para a membrana de Cook no ponto $B\left(x_{1}=\right.$ $\left.48, x_{2}=52\right) \ldots \ldots \ldots \ldots \ldots \ldots \ldots$

6.16 Resultados de rotações para a membrana de Cook no ponto $B\left(x_{1}=\right.$ $\left.48, x_{2}=52\right) \ldots \ldots \ldots \ldots \ldots \ldots \ldots \ldots \ldots \ldots \ldots \ldots \ldots$

6.17 Modelo para a viga com alta razão de aspecto. As propriedades mecânicas são $E=1 \mathrm{MPa}, \nu=0.3$. A resultante de carregamento na ponta da viga tem valor $P=1 \mathrm{~N}$ e a espessura é $t=1 \mathrm{~m} . \ldots . . . .$.

6.18 Exemplo de malha para a viga com alta razão de aspecto contendo 202 elementos de contorno.

6.19 Resultados de deslocamentos $u_{2}$ para a viga com alta razão de aspecto na extremidade livre. . . . . . . . . . . . . . . . . .

6.20 Resultados de rotações $\omega_{3}$ para a viga com alta razão de aspecto na extremidade livre. . . . . . . . . . . . . . . . . .

6.21 Convergência para o deslocamento $u_{2}$ para o caso da viga com alta razão de aspecto e 2 sub regiões. 
6.22 Convergência para a rotação $\omega_{3}$ para o caso da viga com alta razão de aspecto e 2 sub regiões. . . . . . . . . . . . . . . . . . . . .

6.23 Convergência para o deslocamento $u_{2}$ para o caso da viga com alta razão de aspecto e 4 sub regiões. . . . . . . . . . . . . . . . . . . . .

6.24 Convergência para a rotação $\omega_{3}$ para o caso da viga com alta razão de aspecto e 4 sub regiões. . . . . . . . . . . . . . . . . . . . . .

6.25 Análise de convergência dos deslocamentos $u_{2}$ para a viga com alta razão de aspecto considerando diferentes números de pontos de integração. . .

6.26 Análise de convergência das rotações $\omega_{3}$ para a viga com alta razão de aspecto considerando diferentes números de pontos de integração. . . . .

7.1 Dimensões e condições de contorno para a estrutura em "L" [3]. . . . . .

7.2 Distribuição do deslocamento total para a estrutura em "L" obtidos com a formulação deste trabalho.

7.3 Distribuição do deslocamento total para a estrutura em "L" obtidos com a formulação convencional do MEC. . . . . . . . . . . . . . . . . . .

7.4 As dimensões e condições de contorno para a estrutura unida por um

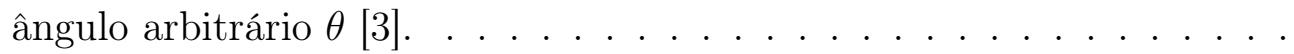

7.5 Deslocamento total para a estrutura unida por um ângulo $\theta=91^{\circ}$ obtido com o MEC convencional.

7.6 Deslocamento total para a estrutura unida por um ângulo $\theta=91^{\circ}$ obtido com a formulação deste trabalho.

7.7 Deslocamento total para a estrutura unida por um ângulo $\theta=95^{\circ}$ obtido com o MEC convencional. . . . . . . . . . . . . . . . . . . . .

7.8 Deslocamento total para a estrutura unida por um ângulo $\theta=95^{\circ}$ obtido com a formulação deste trabalho.

7.9 Deslocamento total para a estrutura unida por um ângulo $\theta=120^{\circ}$ obtido com o MEC convencional.

7.10 Deslocamento total para a estrutura unida por um ângulo $\theta=120^{\circ}$ obtido com a formulação deste trabalho.

7.11 Dimensões e condições de contorno para a viga em forma de caixa em balanço. . . . . . . . . . . . . . . . . .

7.12 Deslocamento total para a viga em formato de caixa em balanço para $L_{3}=0.1 \mathrm{~m}$.

7.13 Deslocamento na direção $x_{3}$ para a viga em formato de caixa em balanço para $L_{3}=0.05 \mathrm{~m}$. . . . . . . . . . . . . . .

7.14 Deslocamento na direção $x_{3}$ para a viga em formato de caixa em balanço para $L_{3}=0.1 \mathrm{~m} \ldots \ldots \ldots \ldots \ldots \ldots \ldots$ 
7.15 Deslocamento na direção $x_{3}$ para a viga em formato de caixa em balanço para $L_{3}=0.2 \mathrm{~m} \ldots \ldots \ldots \ldots$. . . . . . . . . . . 98

7.16 Dimensões e condições de contorno para a viga em forma de caixa em balanço sujeita a um momento em sua extremidade livre. . . . . . . . . 98

7.17 Deslocamento total para a viga em formato de caixa em balanço sujeita a um momento para $L_{3}=0.1 \mathrm{~m}$. . . . . . . . . . . . . . . . . .

7.18 Deslocamentos em $x_{2}=2 \mathrm{~m}$ para a viga em formato de caixa em balanço sujeita a um momento para $L_{3}=0.05 \mathrm{~m}$. . . . . . . . . . . . . . . . . . 100

7.19 Deslocamentos em $x_{2}=2 \mathrm{~m}$ para a viga em formato de caixa em balanço sujeita a um momento para $L_{3}=0.1 \mathrm{~m}$. . . . . . . . . . . . . . . . . 100

7.20 Deslocamentos em $x_{2}=2 \mathrm{~m}$ para a viga em formato de caixa em balanço sujeita a um momento para $L_{3}=0.2 \mathrm{~m}$. . . . . . . . . . . . . . . . . . 101

7.21 Deslocamentos $u_{2}$ para o lado alinhado com a direção $x_{2}$ localizados nas coordenadas $x_{1}=x_{3}=0 \mathrm{~m}$ para $L_{3}=0.05 \mathrm{~m}$. O número de elementos das simulações é indicado na legenda.

7.22 Deslocamentos $u_{2}$ para o lado alinhado com a direção $x_{2}$ localizados nas coordenadas $x_{1}=x_{3}=0 \mathrm{~m}$ para $L_{3}=0.1 \mathrm{~m}$. O número de elementos das simulações é indicado na legenda. . . . . . . . . . . . . . . . . . 103

7.23 Deslocamentos $u_{2}$ para o lado alinhado com a direção $x_{2}$ localizados nas coordenadas $x_{1}=x_{3}=0 \mathrm{~m}$ para $L_{3}=0.2 \mathrm{~m}$. O número de elementos das simulações é indicado na legenda. . . . . . . . . . . . . . 103

7.24 L-shaped structure, $L_{1}=1.0 \mathrm{~m}, L_{2}=2.0 \mathrm{~m}, L_{3}=1.0 \mathrm{~m}, t=0.1 \mathrm{~m}$ (espessura da placa). $E=100 \mathrm{kPa}, \nu=0$ e $q=10 \mathrm{~N}$. . . . . . . . 104

7.25 Resultados obtidos com a formulação do MEC apresentada nesta tese. . 105

7.26 Resultados obtidos com a formulação do MEC convencional. . . . . . . 105

7.27 Resultados obtidos usando o MEF através do ANSYS com o elemento Shell181. . . . . . . . . . . . . . . . . . 106

7.28 Resultados para a placa superior projetados no plano $x_{1}-x_{2}$. . . . . 106 


\section{Lista de Tabelas}

3.1 Quantidade de pontos de Gauss de acordo com o grau do polinômio que pode ser integrado exatamente. . . . . . . . . . . . . . . . 45

6.1 Comparação dos resultados para o ponto $A\left(x_{1}=48, x_{2}=0\right)$, usando 280 nós. . . . . . . . . . . . . . . . . . . . . . . . . 72

6.2 Resultados obtidos para a viga curta. . . . . . . . . . . . . . . . . 74

6.3 Resultados para a membrana de Cook usando 320 pontos de colocação. Deslocamentos verticais e drilling rotations no ponto $B\left(x_{1}=48, x_{2}=52\right) .80$

6.4 Resultados para a membrana de Cook. . . . . . . . . . . . . . . 80

6.5 Resultados para a viga com alta razão de aspecto em $x_{1}=100 \mathrm{~m}$. . . . 83

6.6 Resultados para a viga com alta razão de aspecto. Deslocamentos verticais e rotações na extremidade livre. . . . . . . . . . . . . . . . . . . 83

7.1 Deslocamento na direção $x_{3}$ calculado na ponta da placa comparado às soluções analíticas.

7.2 Deslocamentos calculados para um ponto na extremidade carregada da placa superior comparados com a solução analítica para os três diferentes ângulos. . . . . . . . . . . . . . . . . . . .

7.3 Erro calculado para cada uma das configurações em relação às teorias de viga de Bernouli-Euler e Timoshenko. . . . . . . . . . . . . . . . .

7.4 Deslocamentos $u_{2}$ máximos obtidos com o MEC comparados à solução analítica de Bernoulli-Euler. . . . . . . . . . . . . . . . . . . 102 


\section{Lista de Símbolos}

\section{Letras gregas}

$\alpha$ - Índice variando de 1 a 2.

$\beta$ - Índice variando de 1 a 2.

$\gamma$ - Índice variando de 1 a 2 , parâmetro de penalidade.

$\delta$ - Delta de dirac, delta de Kronecker ou operador variacional.

$\varepsilon$ - Tensor de deformações ou símbolo de permutação.

$\theta-\hat{A}$ ingulo

$\lambda$ - Constante de Lamé, fator de cisalhamento (placas).

$\mu$ - Módulo de cisalhamento.

$\nu$ - Coeficiente de Poisson.

$\xi$ - Coordenada paramétrica ou intrínseca.

$\sigma-$ Tensor de tensões.

$\chi$ - Deformação de flexão (placas).

$\psi$ - Deformação cisalhante (placas).

$\omega$ - Rotações.

$\Gamma$ - Contorno.

$\Xi$ - Matriz de funções de forma para vetores com três componentes.

$\Pi_{c}-$ Trabalho realizado no contorno (placas).

$\Pi_{d}$ - Energia de deformação (placas).

$\Phi$ - Matriz de funções de forma para vetores com duas componentes.

$\Psi$ Rotação da formulação do MEC com funções de forma enriquecidas.

$\Omega$ - Domínio.

$\nabla$ - Laplaciano.

\section{Letras arábicas}

b - Forças de corpo.

$e$-Versor unitário.

$h$ - Espessura de uma placa.

$i$ - Índice variando de 1 a 3.

$j$ - Índice variando de 1 a 3 .

$k$ - Índice variando de 1 a 3.

$n$ - Vetor normal.

$p$ - Forças de superfície generalizadas. 
$q$ - Carga de domínio da formulação de placas.

$t$ - Forças de superfície, vetor unitário tangente ao contorno ou espessura de uma placa.

$u$ - Deslocamentos.

$v$ - vetores unitários das coordenadas locais de placas associadas.

$x$ - Coordenadas.

$A$ - Área.

$C$ - Matriz de rigidez completa.

$C_{n}$ - Constante presente na formulação de placas.

$C_{s}$ - Constante presente na formulação de placas.

$C_{i j}$ - Termos livres das formulações de placa e elasticidade plana.

$C_{i j}$ - Matriz devido às condições de compatibilidade.

$D$ - Combinação linear de derivadas da solução fundamental para deslocamentos (Elasticidade plana) e Constante presente na formulação de placas.

$E$ - Módulo de elasticidade.

$E_{i j}$ - Matriz devido às condições de equilíbrio.

$F$ - Força genérica.

G, $G$ - Vetor de Galerkin, Módulo de cisalhamento.

$I$ - Momento de inércia.

$J$ - Jacobiano da transformação entre o espaço paramétrico e o espaço cartesiano.

$K_{0}, K_{1}$ - Funções de Bessel modificadas.

$L$ - Medida de comprimento, operador diferencial das equações de Navier generalizadas.

$M-$ Momentos fletores.

$N$ - Funções de forma.

$P$ - Ponto genérico, carregamentos ou Solução fundamental para forças de superfície generalizadas (Placas).

$Q$ - Força cisalhante.

$R$ - Respostas numéricas ou analíticas.

$S$ - Combinação linear de derivadas da solução fundamental para forças de superfície (Elasticidade plana).

$T$ - Solução fundamental para forças de superfície (Elasticidade plana) ou matriz de transformação.

$U$ - Solução fundamental para deslocamentos (Elasticidade plana).

$W$ - Solução fundamental para deslocamentos generalizados (Placas).

X - Ponto localizado no domínio. 


\section{Lista de Abreviações}

MEC - Método dos Elementos de Contorno

MEF - Método dos Elementos Finitos

MDF - Método das Diferenças Finitas

ACA - Aproximação Cruzada Adaptativa

GDL - Grau de Liberdade

PUBEM - Partition of Unity Boundary Element Method

XBEM - Extended Boundary Element Method XFEM - Extended Finite Element

Method BE - Bernoulli - Euler 


\section{INTRODUÇÃO}

A necessidade de projetar teve início com as primeiras tentativas do ser humano em alterar o seu ambiente de forma a torná-lo mais favorável. Inicialmente, estruturas já existentes na natureza eram aproveitadas, como cavernas para proteção, pedras e pedaços de madeira como armas etc. De maneira a torná-las mais eficientes, o homem inventou maneiras de alterá-las, por exemplo, atritando uma pedra de maneira a tornar sua borda mais afiada, facilitando o uso para o corte. Estruturas rudimentares desenvolvidas para agricultura, assim como o uso de animais, também foram determinantes para que fosse possível deixar o nomadismo e se fixar em um local. Até este ponto, casas e edificações eram feitas com materiais primitivos e eram totalmente dependentes das habilidades manuais daquele que as estivesse construindo. Mais tarde, com a evolução da técnica, ocorrida em diferentes momentos dependendo da civilização, os procedimentos usados na construção foram padronizados, tornando-os cada vez mais seguros e melhorando a durabilidade daquilo que se estivesse construindo. Destas necessidade básicas, surgiu a demanda pelo estabelecimento de uma ciência como a engenharia que definisse ferramentas de cálculo e padronizações. Atualmente demandas criadas a partir da evolução da humanidade levaram ao projeto de equipamentos, máquinas e estruturas cada vez mais audaciosas que exigem um nível extremamente alto de estudos e técnicas. Como exemplos temos os arranha-céus, ônibus espaciais, geradores hidrelétricos, carros, aviões e etc. Na Figura 1.1 vemos uma destas aplicações.

Grande parte da efetividade destes projetos vem da correta aplicação de técnicas para escolha de materiais e das dimensões. Para que seja possível dizer se uma estrutura é segura, devem ser identificados a que espécies de esforços ela está submetida e como ela suportará essa carga. Essas definições passam pelo entendimento de como esses esforços são distribuídos na estrutura e também do conhecimento da resistência do material do qual ela é construída.

Os primeiros estudos que relacionam a aplicação de forças às deformações foram feitos por volta de 1678 por Robert Hooke, cujo sobrenome é extremamente familiar à maioria dos estudantes das engenharias mecânica, civil e relacionadas. Apesar desse longínquo início, grandes avanços na teoria da elasticidade não foram obtidos até o ano de 1821 no qual Navier publicou seus estudos acerca das equações de equilíbrio. Este desenvolvimento foi seguido por Cauchy que estudou as equações básicas da elasticidade e desenvolveu o conceito de tensão em um ponto. Vários outros cientistas cujos nomes figuram nos livros de teoria da elasticidade continuaram o desenvolvimento desta disciplina, entre os principais podemos citar, Bernoulli, Lord Kelvin, Poisson, Lamé, 


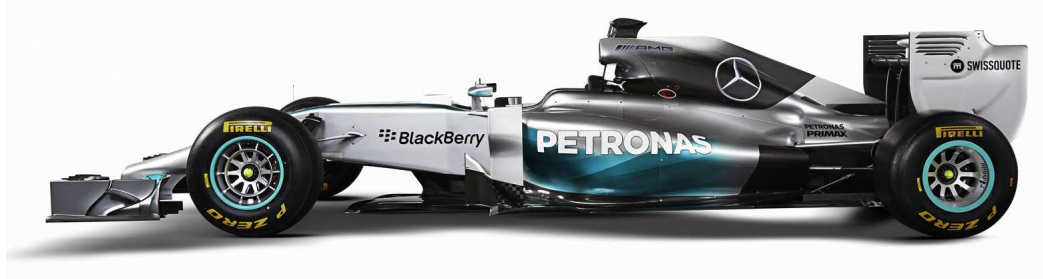

Figura 1.1: Carro de automobilismo da categoria Fórmula 1, exemplo de projeto com utilização das técnicas e materiais mais avançados.

Green, Saint-Venant, Betti, Airy, Kirchhoff, Rayleigh, Love, Timoshenko. A totalidade destes autores desenvolveu a forte base matemática presente nos livros e formulações usadas atualmente, sendo muito dos seus desenvolvimentos homenageados em denominações como a viga de Timoshenko, teoria de placas de Kirchhoff-Love, princípio de Saint-Venant, coeficiente de Poisson, solução fundamental de Kelvin para elasticidade [1].

A partir dos anos 70 uma poderosa ferramenta despontou no mundo da engenharia, o computador. A potencialidade de realizar repetidos cálculos em um tempo pequeno e de sistematizar certas atividades, levou os pesquisadores a aplicar alguns conceitos como análise matricial de estruturas a uma configuração mais abrangente. Surgiram assim os métodos numéricos como o Método dos Elementos Finitos (MEF), o Método das Diferenças Finitas (MDF) e o Método dos Elementos de Contorno (MEC). As equações das formulações analíticas levam a uma grande dificuldade de solução para geometrias e carregamentos que não sejam matematicamente bem comportados, assim a maioria das soluções anteriores para problemas práticos, partiu de generosas simplificações das situações reais. As técnicas numéricas expandiram a possibilidade de aplicações e não só facilitaram o dimensionamento de peças, como agilizaram imensamente o processo. O incremento do poder computacional dos computadores também foi decisivo para a sua aplicação em larga escala e está intimamente ligado a sua evolução.

O Método dos Elementos de Contorno (MEC), entre outras técnicas, tem sido fonte de pesquisas científicas ao longo das últimas décadas. Seu uso, em comparação ao Método dos Elementos Finitos (MEF), contudo é consideravelmente menor. Isso se deve à versatilidade e adaptabilidade do MEF e a extensa pesquisa realizada, que 
o tornou um método robusto e deu origem a vários programas comerciais, como o Ansys, Nastran, Abaqus etc. Apesar disso, o MEC oferece algumas vantagens, entre elas temos, a redução da dimensão do problema, uma vez que apenas o contorno é discretizado. Um esforço computacional menor quando da reconstrução de uma malha, vantagens em problemas onde o fenômeno de interesse é predominantemente localizado no contorno. Também podem ser citadas desvantagens entre as quais, dificuldades no tratamento de problemas não-lineares, um sistema de equações resultantes formado por uma matriz cheia e não simétrica, cujo tempo de solução cresce bastante com o tamanho do problema. Esta última característica faz com que, em seu estado puro, o MEC não seja uma técnica adequada a problemas de larga escala. O problema do tempo computacional do MEC pode ser atenuado atualmente, através da aplicação daqueles que ficaram conhecidos como métodos rápidos dos elementos de contorno. Entre eles, se destacam a expansão em multi pólos [4] e a Aproximação Cruzada Adaptativa (ACA) [5].

As estruturas são comumente classificadas de acordo com suas características físicas e às vezes no modo como são carregadas. Como exemplo temos as barras e vigas que podem possuir o mesmo formato mas se carregadas axialmente recebem a primeira classificação e se fletidas, a segunda. Este caso é análogo ao de chapas e placas. Neste caso a chapa seria um equivalente bidimensional da barra e as placas das vigas. Outros tipos são os arranjos conhecidos como treliças, pórticos e grelhas, cada um com suas particularidades quanto ao tipo de deslocamentos e carregamentos. A análise desse tipo de estrutura é importante uma vez que sua aplicação prática é muito comum e o desenvolvimento de peças e componentes mais resistentes e leves é uma demanda constante. Esses tipos de configuração são aplicadas nas mais diversas áreas, desde a engenharia civil, com coberturas e telhados sustentados por treliças, lages em prédios, vigas sustentadoras de edifícios e muitos outras. Nas telecomunicações e distribuição de energia com estruturas de antenas e torres de transmissão. Na engenharia mecânica como fuselagens de aviões, chassis automobilísticos e muitos outros.

\subsection{Revisão Bibliográfica}

Nesta tese o tema da associação de placas espessas através do MEC será abordado e a inclusão da rotação $\omega_{3}$ será o seu foco. Para tanto, os seguinte temas serão revisados:

- As teorias desenvolvidas para placas e elementos planos como chapas.

- A drilling rotation e seu desenvolvimento em outros métodos numéricos.

- A associação de placas no MEC e suas limitações. 
As teorias clássicas para placas não são suficientes para uma descrição precisa de seu comportamento quando a relação entre espessura e a maior aresta é relativamente grande. Essa ocorrência é devido ao negligenciamento de deformações cisalhantes presentes no fenômeno, por isso as teorias de placas deformáveis por cisalhamento, apesar de mais complexas, são preferíveis no caso de uma formulação com maior aplicabilidade.

A primeira teoria para descrição do comportamento de placas foi proposta por Kirchhoff [6] em 1850. Ela ficou conhecida como teoria de placas finas e é suficientemente precisa para a maioria das aplicações práticas. Em alguns casos, porém, ela é comprovadamente falha, mesmo que as placas tratadas não sejam classificadas como espessas. São exemplos de situações como estas, placas que possuem concentradores de tensão e aquelas anisotrópicas com grande diferenças em suas propriedades direcionais.

Uma teoria mais abrangente foi proposta por Reissner [7] em 1947. Essa teoria assume uma variação da tensão ao longo da espessura da placa, levando em consideração assim também uma variação das deformações cisalhantes. Por partir de uma suposição relacionada à tensão, é dita como sendo uma teoria de placas deformáveis por cisalhamento baseada na tensão. A teoria de Mindlin [8] foi proposta com base em uma suposição de um campo de deslocamentos variável através da espessura da placa. De maneira semelhante ao caso da placa de Reissner, como partiu da suposição de variação linear dos deslocamentos ao longo espessura, esta é dita como teoria de placas deformáveis por cisalhamento baseada nos deslocamentos. Teorias de mais alta ordem também foram obtidas, como a teoria de Reddy onde variações quadráticas das deformações cisalhantes são assumidas [9]. Essa é uma teoria de segunda ordem.

Formulações do MEC para placas estão bastante estabelecidas com soluções fundamentais para a teoria de Kirchhoff e para as teorias de Mindlin e Reissner [10]. Um dos primeiros trabalhos utilizando o MEC com estas formulações foi feito por Weeën [11]. Seu desenvolvimento completo, contendo o tratamento de singularidades, imposição de condições de contorno e cálculo de tensões é descrito em vários livros [12, 13]. Inúmeros trabalhos publicados tratam de aplicações específicas como formulações dinâmicas $[14,15]$ e anisotropia $[16,17,18]$.

As formulações de elasticidade plana foram desenvolvidas nos primórdios do MEC e são facilmente encontradas em vários livros textos comuns aos estudantes iniciantes no assunto. Podemos incluir aqui, além daquelas citadas acima, as referências [19, 20]. Um dos primeiros trabalhos a tratar de uma formulação do MEC para elasticidade tridimensional foi publicado no artigo [21].

Para que seja possível lidar com um problema de associação de placas, que é essencialmente tridimensional, com uma formulação bidimensional é necessário tratar cada placa como uma subregião do problema. O problema global é então montado após a 
imposição das condições de compatibilidade e equilíbrio em arestas comuns entre estas placas. Para o caso de duas regiões que compartilham o mesmo plano, o procedimento é bastante simplificado. Contudo no caso de placas associadas contidas em planos genéricos, uma dificuldade surge durante a imposição das condições de compatibilidade, por conta da ausência de uma rotação na direção $x_{3}$ local. O procedimento comumente adotado foi criar restrições que permitissem a solução do problema em casos específicos. A geometria deveria ter as arestas que uniam as placas alinhadas a um dos eixos do problema e os carregamentos não poderiam provocar um desalinhamento com essa direção após a deformação. Dessa maneira as condições de compatibilidade poderiam partir do princípio que a rotação em questão, tem um valor nulo, evitando assim os erros de não considerá-la na formulação. São exemplos de trabalhos que adotaram essas restrições $[22,23,3,24]$. Essa dificuldade pode ser superada com a inclusão da rotação $\omega_{3}$ que é diretamente relacionada aos deslocamentos da elasticidade plana $u_{1}$ e $u_{2}[25]$.

Uma das abordagens utilizadas por pesquisadores que trabalham com o MEF para tratar de estruturas formadas por placas ou cascas envolve a obtenção de um elemento finito através da combinação adequada de elementos finitos de membrana e placas. As Formulações do MEC criadas para esta situação se baseiam em um procedimento semelhante, no qual são combinadas as equações integrais das duas formulações, dando origem a uma formulação acoplada, que considera os efeitos fletores e de extensão da placa. Como mostrado no parágrafo anterior, a ausência de uma rotação em torno do eixo $x_{3}$ causa dificuldades numéricas no tratamento desse tipo de estrutura. No caso do MEF, essa ausência causa o aparecimento de singularidades na matriz de rigidez, o que foi solucionado usando elementos de membrana que continham esse grau de liberdade adicional [26]. No caso do MEC esta questão foi aparentemente ignorada até o momento.

Inicialmente, a rotação $\omega_{3}$, também conhecida como drilling rotation, foi incluída apenas com o intuito de melhorar a precisão do elemento finito e acelerar a sua convergência, sendo assim uma alternativa mais barata do ponto de vista computacional. Em 1984 Allman [27] desenvolveu um elemento finito triangular que representava o deslocamento com uma variação quadrática usando apenas conectores nos vértices, dois deslocamentos e uma rotação. Isto resultou em deformações e tensões com distribuição linear ao longo do elemento. Esta foi uma das primeiras tentativas bem sucedidas de inclusão desta rotação no MEF, na sequência outro elemento foi desenvolvido por [28] usando a formulação livre de Bergan e Nygård [29].

Outros autores, abordaram o problema usando uma variedade de metodologias como [30, 31, 32, 33]. Todos eles se basearam em uma alteração da função de forma, 
feita de modo a incluir o grau de liberdade ( $g d l)$ adicional de rotação, seguindo o trabalho inicial publicado por Allman [27]. Este procedimento foi realizado em conjunto com uma estratégia de impor que esse gdl incluído se igualasse à rotação da teoria da elasticidade. Em [34] os autores descobriram que o triângulo de Allman toma uma forma típica de aproximação por partição da unidade, o que permite uma extensão direta para métodos sem malha e de contorno. Neste trabalho foram apresentados resultados para o MEF e para Element-free Galerkin Method.

Um dos primeiros trabalhos a tratar do tema na comunidade do MEC utilizou o procedimento descrito em [34], tratado no parágrafo anterior. Esse trabalho foi publicado em [25]. O procedimento adotado, utilizou elementos de contorno lineares descontínuos, contínuos e semi descontínuos. A inclusão da rotação se deu através da função de forma o que levou o sistema de equações resultante a ter uma inconsistência entre número de variáveis e equações. Esse problema foi contornado com inclusão de um funcional que quando minimizado, igualava a rotação recém incluída àquela da teoria da elasticidade. Este procedimento resultou em bom acordo com resultados disponíveis na literatura e soluções analíticas.

A tática adotada nesta tese é independente da função de forma e usa as expressões analíticas do problema no ponto anterior a sua discretização. De tal maneira que para incluir o sexto grau de liberdade na formulação acoplada (placas e membranas), uma expressão foi obtida através de derivadas da equação integral de contorno para deslocamentos na elasticidade plana e da definição da rotação oriunda da teoria da elasticidade. Esta nova equação é então incluída nas matrizes de influência da formulação do MEC para elasticidade plana e é diretamente dependente dos deslocamentos e forças de superfície. Duas das principais vantagens da presente abordagem em relação a outras técnicas numéricas, como o MEF, são as expressões analíticas obtidas para a rotação $\omega_{3}$ que é aproximada apenas durante a discretização do problema, e sua facilidade em ser estendida para elementos de mais alta ordem [35]. Os resultados obtidos aqui, apresentam também uma melhor precisão quando comparados com aqueles obtidos em [25]. Como desvantagens, temos a necessidade da integração de equações hiper singulares, que devem ser tratadas adequadamente.

Utilizando o método descrito acima, neste trabalho, o MEC é aplicado na formulação para associação de placas que originalmente apresenta cinco graus de liberdade, dois oriundos da elasticidade plana e três das placas deformáveis por cisalhamento. Esse procedimento padrão, baseado na combinação dos comportamentos extensores e fletores, é acoplado em um único sistema de equações [3]. Tanaka et al. [22, 23] utilizou também este procedimento para simular a vibração livre de estruturas elásticas formadas por placas com o MEC. Os dois trabalhos utilizaram o método de sub regiões do 
MEC o que lhes permitiu gerar um sistema de equações em coordenadas locais e impor condições de compatibilidade e equilíbrio, transformando-as para o sistema de referência global. Com a inclusão do sexto grau de liberdade nesta formulação, através do procedimento anterior, foram obtidos resultados numéricos para várias configurações que foram comparados com respostas analíticas e obtidas com o Ansys.

\subsection{Divisão do trabalho}

Esta tese está dividida em 8 capítulos, que abordam os seguintes tópicos:

1. Introdução ao assunto, revisão bibliográfica e divisão do trabalho;

2. A teoria da elasticidade voltada aos problemas de elasticidade plana e triaxial, e equacionamento de placas deformáveis por cisalhamento;

3. A formulação do MEC para a elasticidade plana e placas deformáveis por cisalhamento;

4. Obtenção da formulação do MEC para a rotação $\omega_{3}$ através de duas abordagens distintas;

5. Formulação para associação de placas e aspectos relativos à implementação;

6. Resultados numéricos obtidos para a formulação plana com enfoque nas respostas para $\omega_{3} ;$

7. Resultados numéricos obtidos para a formulação de associação de placas;

8. Conclusões e propostas para trabalhos futuros. 


\section{TEORIA DA ELASTICIDADE}

\subsection{Introdução}

A Elasticidade é um ramo da ciência que trata, principalmente da determinação das tensões, deformações e distribuição de deslocamentos em um sólido elástico sob a influência de carregamentos. Para tal, modelos matemáticos foram desenvolvidos possibilitando a análise de problemas com aplicações práticas e teóricas na engenharia. Como exemplos destas, podem ser citados:

- Análise estrutural no âmbito da engenharia civil com a determinação de tensões e deformações em elementos, como placas, cascas, vigas e aplicações em geo mecânica envolvendo a análise de solo, rochas e concreto.

- Análise estrutural no âmbito da engenharia mecânica como o projeto de peças e partes de máquinas e equipamentos, para as quais são levadas em consideração a análise de tensões, problemas de contato, fratura e fadiga.

- Na ciência dos materiais, com a determinação de campos de tensão em sólidos cristalinos nas proximidades de uma discordância.

- Aplicações em engenharia aeronáutica e aeroespacial.

A teoria desta disciplina também constitui a base para estudos mais avançados como plasticidade e viscoelasticidade, além do emprego de métodos computacionais para a solução de problemas com geometrias e carregamentos mais complicados [1].

Nesta seção a teoria da elasticidade será abordada cobrindo os aspectos básicos das relações entre tensão e deformação, relações cinemáticas e condições de equilíbrio onde as definições serão particularizadas para o caso da Elasticidade Plana e de Placas Espessas. A partir deste capítulo e ao longo do restante desta tese a notação indicial será utilizada. Índices com letras gregas $(\alpha, \beta)$ terão variação de 1 a 2 , enquanto índices com letras romanas terão variação de 1 a 3.

\section{$2.2 \quad$ Elasticidade}

Nesta seção vários conceitos da teoria da Elasticidade serão tratados e ao final, considerações serão feitas para que sejam especificadas para o caso da Elasticidade Plana. 


\subsubsection{Relação entre deformações e deslocamentos}

Os sólidos elásticos mudam de forma, ou seja, deformam quando sujeitos a um carregamento. Essas deformações podem ser quantificadas uma vez que seja conhecido o campo de deslocamentos por todo o corpo. Deve ser levado em consideração que a hipótese de um meio contínuo estabelece que há um campo de deslocamentos abrangendo todos os pontos de um sólido elástico. Na Figura 2.1 é mostrado um exemplo de barra sofrendo uma deformação sobre a ação de um carregamento axial.

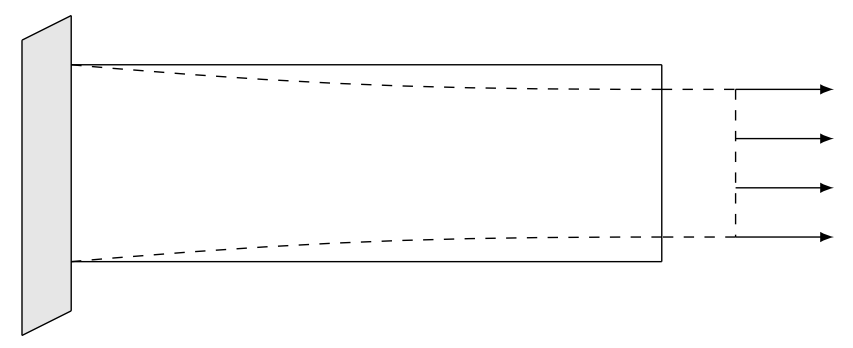

Figura 2.1: Exemplo de barra sofrendo uma deformação sobre a ação de um carregamento axial.

Considere agora a Figura 2.2. Nela têm-se dois pontos $P_{0}$ e $P$ ligados por um vetor de posição relativa $\mathbf{r}$ na configuração inicial. Após o corpo em questão sofrer uma deformação, estes pontos passam a ocupar as posições $P_{0}^{\prime}$ e $P^{\prime}$ e são ligados por um vetor $\mathbf{r}^{\prime}$. Para o caso elástico de pequenas deformações, a diferença entre as configurações pode ser ignorada [1]. Considerando essa análise, um corpo elástico sólido é dito deformado quando a distância relativa entre dois pontos quaisquer se altera, o que não acontece no chamado movimento de corpo rígido.
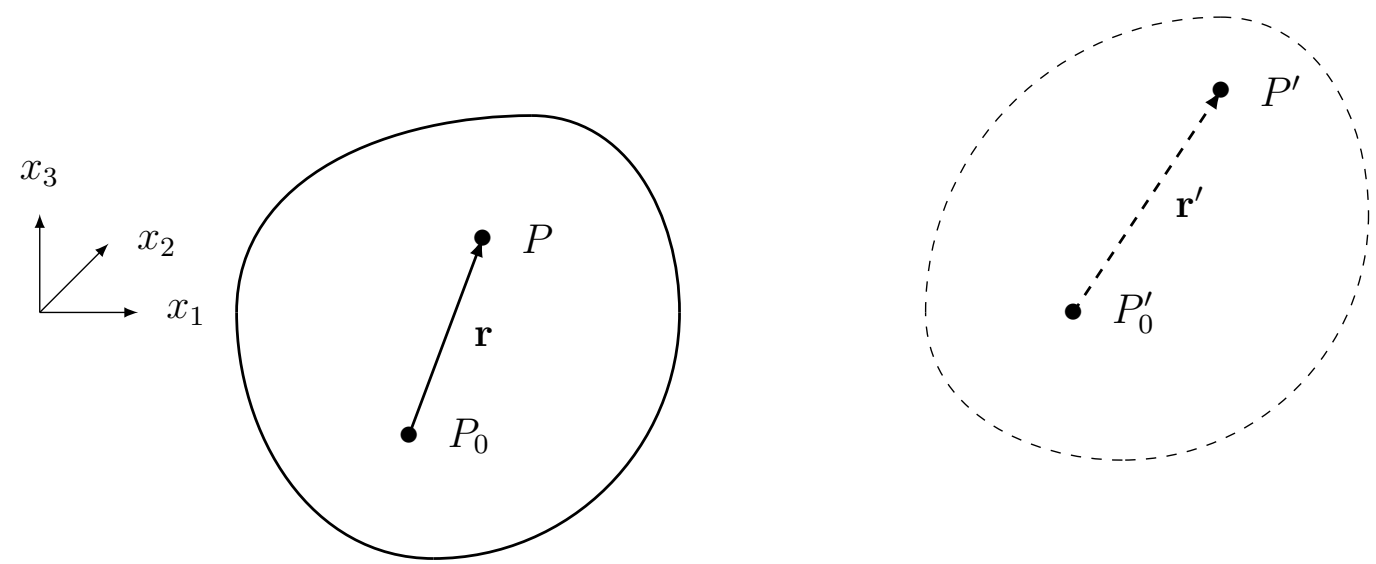

Figura 2.2: Deslocamento de um ponto dentro de um corpo elástico sólido, inicialmente na configuração não deformada em linha cheia e na deformada em linha tracejada [1].

Usando coordenadas cartesianas e definindo vetores de deslocamento $\mathbf{u}^{0}$ de $P_{0}$ para $P$ e u de $P_{0}^{\prime}$ para $P^{\prime}$. Considerando também que estes pontos são vizinhos, é pos- 
sível usar uma expansão em série de Taylor ao redor do ponto $P_{0}$ para expressar as componentes de $\mathbf{u}$, como mostrado na equação (2.1):

$$
\begin{aligned}
& u_{1}=u_{1}^{0}+\frac{\partial u_{1}}{\partial x_{1}} r_{1}+\frac{\partial u_{1}}{\partial x_{2}} r_{2}+\frac{\partial u_{1}}{\partial x_{3}} r_{3} \\
& u_{2}=u_{2}^{0}+\frac{\partial u_{2}}{\partial x_{1}} r_{1}+\frac{\partial u_{2}}{\partial x_{2}} r_{2}+\frac{\partial u_{2}}{\partial x_{3}} r_{3} \\
& u_{3}=u_{3}^{0}+\frac{\partial u_{3}}{\partial x_{1}} r_{1}+\frac{\partial u_{3}}{\partial x_{2}} r_{2}+\frac{\partial u_{3}}{\partial x_{3}} r_{3}
\end{aligned}
$$

onde $u_{i}$ são as componentes do vetor de deslocamentos. A mudança do vetor de posição relativa pode ser escrita como:

$$
\Delta \mathbf{r}=\mathbf{r}^{\prime}-\mathbf{r}=\mathbf{u}^{\prime}-\mathbf{u}^{0}
$$

o que, a partir de (2.1), resulta em:

$$
\begin{aligned}
\Delta r_{1} & =\frac{\partial u_{1}}{\partial x_{1}} r_{1}+\frac{\partial u_{1}}{\partial x_{2}} r_{2}+\frac{\partial u_{1}}{\partial x_{3}} r_{3} \\
\Delta r_{2} & =\frac{\partial u_{2}}{\partial x_{1}} r_{1}+\frac{\partial u_{2}}{\partial x_{2}} r_{2}+\frac{\partial u_{2}}{\partial x_{3}} r_{3} \\
\Delta r_{3} & =\frac{\partial u_{3}}{\partial x_{1}} r_{1}+\frac{\partial u_{3}}{\partial x_{2}} r_{2}+\frac{\partial u_{3}}{\partial x_{3}} r_{3}
\end{aligned}
$$

ou em notação indicial

$$
\Delta r_{i}=u_{i, j} r_{j} \quad i, j=1,2,3 .
$$

O termo $u_{i, j}$ é o tensor do gradiente dos deslocamentos e suas componentes são, de acordo com [1]:

$$
u_{i, j}=\left[\begin{array}{lll}
\frac{\partial u_{1}}{\partial x_{1}} & \frac{\partial u_{1}}{\partial x_{2}} & \frac{\partial u_{1}}{\partial x_{3}} \\
\frac{\partial u_{2}}{\partial x_{1}} & \frac{\partial u_{2}}{\partial x_{2}} & \frac{\partial u_{2}}{\partial x_{3}} \\
\frac{\partial u_{3}}{\partial x_{1}} & \frac{\partial u_{3}}{\partial x_{2}} & \frac{\partial u_{3}}{\partial x_{3}}
\end{array}\right]
$$

Este tensor pode ser divido em uma parte simétrica e em uma antissimétrica como mostrado nas equações (2.6) e (2.7):

$$
\begin{aligned}
\varepsilon_{i j} & =\frac{1}{2}\left(u_{i, j}+u_{j, i}\right) \\
\omega_{i j} & =\frac{1}{2}\left(u_{i, j}-u_{j, i}\right)
\end{aligned}
$$

que são conhecidos como os tensores de deformação e de rotação, respectivamente. Um vetor dual pode ser associado ao tensor de rotação usando $\omega_{i}=-1 / 2 \varepsilon_{i k j} \omega_{k j}$. Assim, tem-se: 


$$
\begin{aligned}
& \omega_{1}=\omega_{32}=\frac{1}{2}\left(u_{3,2}-u_{2,3}\right) \\
& \omega_{2}=\omega_{13}=\frac{1}{2}\left(u_{1,3}-u_{3,1}\right) \\
& \omega_{3}=\omega_{21}=\frac{1}{2}\left(u_{2,1}-u_{1,2}\right)
\end{aligned}
$$

Essas componentes representam rotações de corpo rígido em torno dos eixos coordenados e, como tal, não contribuem para o estado de tensão. O vetor $\omega_{3}$ é a rotação de corpo rígido em torno do eixo $x_{3}$, também conhecida como drilling rotation.

A relação entre deformações e deslocamentos ficou estabelecida pela equação (2.6).

\subsubsection{Compatibilidade de deformações}

Com o intuito de garantir que se obtenha uma solução única e contínua para o campo de deformações, é necessário obter algumas relações adicionais. Uma vez que a deformação é diretamente relacionada aos deslocamentos, basta que encontremos um campo de deslocamentos único e contínuo para que o campo de deformações tenha o mesmo comportamento. Porém o mesmo não funciona no sentido inverso, ou seja, um campo de deformações único e contínuo nem sempre produzirá um campo de deslocamentos com as mesmas características. Com o intuito de garantir isso, as deformações devem satisfazer relações conhecidas como condições de compatibilidade.

Para alcançar essa relação, [1] utiliza um processo de eliminação dos deslocamentos das expressões nas quais são estabelecidas sua relação com as deformações. Adotando a notação indicial e derivando a equação (2.6) duas vezes, primeiramente em relação a $x_{k}$ e depois em relação a $x_{l}$, tem-se:

$$
\varepsilon_{i j, k l}=\frac{1}{2}\left(u_{i, j k l}+u_{j, i k l}\right) \quad i, j, k, l=1,2,3 .
$$

e realizando algumas trocas de índices obtêm-se as seguintes relações:

$$
\begin{aligned}
\varepsilon_{k l, i j} & =\frac{1}{2}\left(u_{k, l i j}+u_{l, k i j}\right) \\
\varepsilon_{j l, i k} & =\frac{1}{2}\left(u_{j, l i k}+u_{l, j i k}\right) \\
\varepsilon_{i k, j l} & =\frac{1}{2}\left(u_{i, k j l}+u_{k, i j l}\right)
\end{aligned}
$$

Considerando a continuidade dos deslocamentos, a ordem das derivadas pode ser alterada e as expressões podem ser manipuladas de maneira a resultar em:

$$
\varepsilon_{i j, k l}+\varepsilon_{k l, i j}-\varepsilon_{i k, j l}-\varepsilon_{j l, i k}=0
$$


Estas expressões são conhecidas como equações de compatibilidade de Saint-Venant. Esse sistema leva a 81 equações mas é possível mostrar que muitas delas são idênticas ou repetições e apenas seis delas tem significado prático. Estas seis relações podem ser determinadas fazendo $k=l$, em notação escalar são dadas por:

$$
\begin{gathered}
\frac{\partial^{2} \varepsilon_{11}}{\partial x_{2}^{2}}+\frac{\partial^{2} \varepsilon_{22}}{\partial x_{1}^{2}}=2 \frac{\partial^{2} \varepsilon_{12}}{\partial x_{1} \partial x_{2}} \\
\frac{\partial^{2} \varepsilon_{22}}{\partial x_{3}^{2}}+\frac{\partial^{2} \varepsilon_{33}}{\partial x_{2}^{2}}=2 \frac{\partial^{2} \varepsilon_{23}}{\partial x_{2} \partial x_{3}} \\
\frac{\partial^{2} \varepsilon_{33}}{\partial x_{1}^{2}}+\frac{\partial^{2} \varepsilon_{11}}{\partial x_{3}^{2}}=2 \frac{\partial^{2} \varepsilon_{13}}{\partial x_{3} \partial x_{1}} \\
\frac{\partial^{2} \varepsilon_{11}}{\partial x_{2} \partial x_{3}}=\frac{\partial}{\partial x_{1}}\left(-\frac{\partial \varepsilon_{23}}{\partial x_{1}}+\frac{\partial \varepsilon_{31}}{\partial x_{2}}+\frac{\partial \varepsilon_{12}}{\partial x_{3}}\right) \\
\frac{\partial^{2} \varepsilon_{22}}{\partial x_{3} \partial x_{1}}=\frac{\partial}{\partial x_{2}}\left(-\frac{\partial \varepsilon_{31}}{\partial x_{2}}+\frac{\partial \varepsilon_{12}}{\partial x_{3}}+\frac{\partial \varepsilon_{23}}{\partial x_{1}}\right) \\
\frac{\partial^{2} \varepsilon_{33}}{\partial x_{1} \partial x_{2}}=\frac{\partial}{\partial x_{3}}\left(-\frac{\partial \varepsilon_{12}}{\partial x_{3}}+\frac{\partial \varepsilon_{23}}{\partial x_{1}}+\frac{\partial \varepsilon_{31}}{\partial x_{2}}\right)
\end{gathered}
$$

Pode-se ainda mostrar que as seis equações apresentadas acima não são totalmente independentes e que existe uma relação diferencial entre elas. Contudo, é mais conveniente utilizá-las nesta forma.

\subsubsection{Tensões e equilíbrio}

Nesta seção as definições e propriedades dos vetores de força de superfície e tensor de tensões serão estabelecidas, assim como as equações de equilíbrio de forças. Estas relações são outra parte das equações necessárias para a solução de um problema geral de elasticidade.

A ideia geral por trás da relação entre forças de superfície, é que para que seja mantido o equilíbrio, a aplicação de forças externas induz o aparecimento de forças internas. Elas são convenientemente categorizadas em duas classes:

- Forças de corpo

- Forças de superfície

As forças de corpo são proporcionais à massa do corpo e são resultado de um agente externo. São exemplos a força peso, que é gerada por campos gravitacionais, forças magneticamente induzidas e as inerciais. As forças de superfície, como o nome indica, sempre atuam na superfície do corpo.

Para quantificar a natureza das forças distribuídas dentro de um corpo contínuo sólido, deve-se analisar a ação de um conjunto de carregamentos arbitrários. Uma 

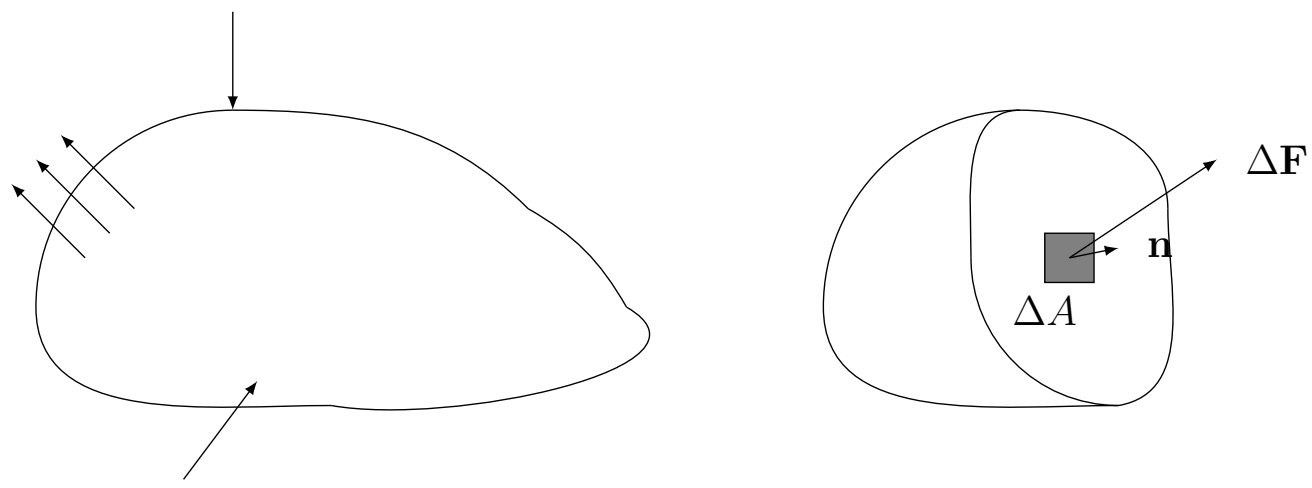

Figura 2.3: Representação de um sólido genérico sob a ação de carregamentos arbitrários.

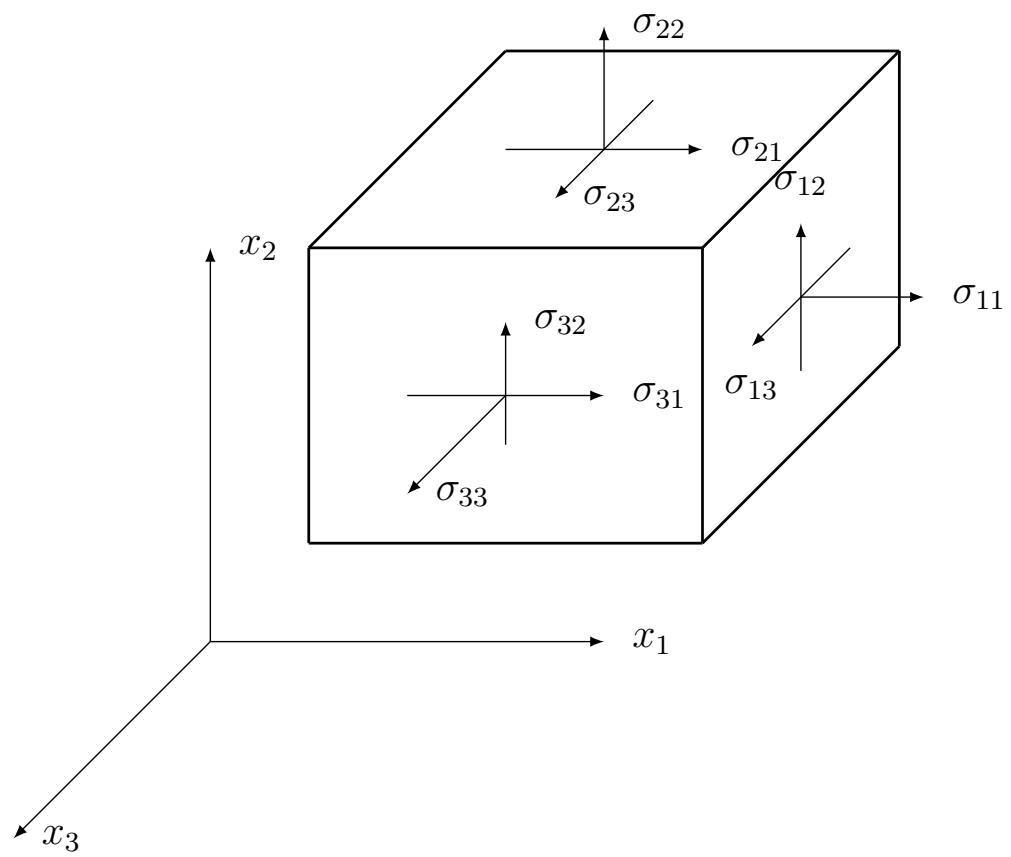

Figura 2.4: Planos coordenados com respectivos valores de tensão.

seção é feita nesse corpo e nela pode-se observar uma área $\Delta A$ com o vetor normal dado por $\mathbf{n}$, na qual atua uma força resultante $\Delta \mathbf{F}$. Dessa maneira o vetor de forças de superfície é definido como:

$$
\mathbf{t}^{n}(\mathbf{x}, \mathbf{n})=\lim _{\Delta A \rightarrow 0} \frac{\Delta \mathbf{F}}{\Delta A}
$$

que é um vetor que também depende da normal, que por sua vez define a orientação da área. Agora considerando a mesma situação, só que dessa vez com $\Delta A$ coincidindo com os três planos formados pelos eixos coordenadas $\left(x_{2}, x_{3}\right),\left(x_{3}, x_{1}\right)$ e $\left(x_{1}, x_{2}\right)$ e alinhados com a direção positiva dos eixos perpendiculares, como mostrado na Figura 2.4. Nesse caso o vetor de forças de superfície, para cada umas das faces, fica: 


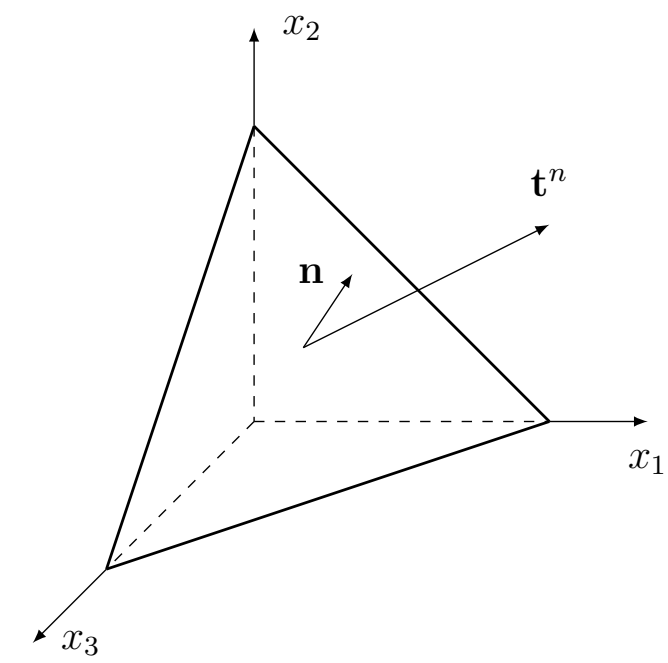

Figura 2.5: Plano oblíquo mostrando normal e uma resultante de força de superfície.

$$
\begin{aligned}
& \mathbf{t}^{n}\left(\mathbf{x}, \mathbf{n}=\mathbf{e}_{1}\right)=\sigma_{11} \mathbf{e}_{1}+\sigma_{12} \mathbf{e}_{2}+\sigma_{13} \mathbf{e}_{3} \\
& \mathbf{t}^{n}\left(\mathbf{x}, \mathbf{n}=\mathbf{e}_{2}\right)=\sigma_{21} \mathbf{e}_{1}+\sigma_{22} \mathbf{e}_{2}+\sigma_{23} \mathbf{e}_{3} \\
& \mathbf{t}^{n}\left(\mathbf{x}, \mathbf{n}=\mathbf{e}_{3}\right)=\sigma_{31} \mathbf{e}_{1}+\sigma_{32} \mathbf{e}_{2}+\sigma_{33} \mathbf{e}_{3}
\end{aligned}
$$

na qual $\mathbf{e}_{1}, \mathbf{e}_{2}$ e $\mathbf{e}_{3}$ são os vetores unitários nas direções dos eixos coordenados. Os nove termos que multiplicam esses vetores são as componentes daquele que é conhecido como o tensor de tensões. Escrito em forma matricial, fica:

$$
\sigma=\left[\begin{array}{lll}
\sigma_{11} & \sigma_{12} & \sigma_{13} \\
\sigma_{21} & \sigma_{22} & \sigma_{23} \\
\sigma_{31} & \sigma_{32} & \sigma_{33}
\end{array}\right]
$$

onde os termos da diagonal com sub índices iguais são as tensões normais, enquanto aqueles de fora da diagonal, com sub índices diferentes, são conhecidos como tensões cisalhantes. Este tensor é simétrico e portanto apresenta apenas 6 componentes distintas.

Analisando agora um vetor de forças de superfície em um plano oblíquo, como o mostrado na Figura 2.5, para o qual o vetor normal pode ser representado por:

$$
\mathbf{n}=n_{1} \mathbf{e}_{1}+n_{2} \mathbf{e}_{2}+n_{3} \mathbf{e}_{3}
$$

onde $n_{1}, n_{2}$ e $n_{3}$ são os cossenos diretores do vetor unitário $\mathbf{n}$. Considerando o equilíbrio deste elemento piramidal em relação as forças atuantes no plano oblíquo e nos planos coordenados, 


$$
\mathbf{t}^{n}=n_{1} \mathbf{t}^{n}\left(\mathbf{n}=\mathbf{e}_{1}\right)+n_{2} \mathbf{t}^{n}\left(\mathbf{n}=\mathbf{e}_{2}\right)+n_{3} \mathbf{t}^{n}\left(\mathbf{n}=\mathbf{e}_{3}\right)
$$

e usando as equações (2.18) a (2.21), pode-se escrevê-lo na seguinte forma:

$$
\begin{aligned}
\mathbf{t}^{n}= & \left(\sigma_{11} n_{1}+\sigma_{21} n_{2}+\sigma_{31} n_{3}\right) \mathbf{e}_{1}+\left(\sigma_{12} n_{1}+\sigma_{22} n_{2}+\sigma_{32} n_{3}\right) \mathbf{e}_{2}+ \\
& \left(\sigma_{13} n_{1}+\sigma_{23} n_{2}+\sigma_{33} n_{3}\right) \mathbf{e}_{3}
\end{aligned}
$$

que em notação indicial e considerando a simetria do tensor de tensões, toma a forma da equação (2.23):

$$
t_{i}^{n}=\sigma_{i j} n_{j}
$$

Esta expressão mostra a relação existente entre a força de superfície e o tensor de tensões.

Seguindo a discussão mostrada em [1], as equações de equilíbrio assumem a seguinte forma:

$$
\sigma_{i j, j}+b_{i}=0
$$

onde $b_{i}$ são as forças de corpo. A equação (2.24) representa as 3 equações envolvendo os campos de tensões que devem ser satisfeitas para que um corpo esteja em equilíbrio estático. Para o caso da Elasticidade Plana, em notação escalar, a equação (2.24) toma a seguinte forma:

$$
\begin{aligned}
& \frac{\partial \sigma_{11}}{\partial x_{1}}+\frac{\partial \sigma_{21}}{\partial x_{2}}+b_{1}=0 \\
& \frac{\partial \sigma_{12}}{\partial x_{1}}+\frac{\partial \sigma_{22}}{\partial x_{2}}+b_{2}=0
\end{aligned}
$$

\subsubsection{Comportamento do material em regime elástico}

Finalmente, para complementar as equações já apresentadas, é necessário que se leve em consideração as propriedades do material analisado. Isso é expressado matematicamente, no caso da elasticidade linear, pela Lei de Hooke que introduz uma relação entre tensões e deformações através das propriedades mecânicas do material em questão. Intuitivamente isso pode ser entendido como até que ponto um material irá se deformar quando sujeito a um determinado esforço e de que maneira esta deformação ocorre. 
A lei de Hooke se baseia em um modelo matemático que considera cada uma das componentes de tensão dependente linearmente de todas as componentes de deformação. A equação (2.27) apresenta essas relações já levando em consideração a simetria dos tensores.

$$
\left\{\begin{array}{l}
\sigma_{11} \\
\sigma_{22} \\
\sigma_{33} \\
\sigma_{12} \\
\sigma_{23} \\
\sigma_{13}
\end{array}\right\}=\left[\begin{array}{llllll}
C_{11} & C_{12} & C_{13} & C_{14} & C_{15} & C_{16} \\
C_{21} & C_{22} & C_{23} & C_{24} & C_{25} & C_{26} \\
C_{31} & C_{32} & C_{33} & C_{34} & C_{35} & C_{36} \\
C_{41} & C_{42} & C_{43} & C_{44} & C_{45} & C_{46} \\
C_{51} & C_{52} & C_{53} & C_{54} & C_{55} & C_{56} \\
C_{61} & C_{62} & C_{63} & C_{64} & C_{65} & C_{66}
\end{array}\right]\left\{\begin{array}{l}
\varepsilon_{11} \\
\varepsilon_{22} \\
\varepsilon_{33} \\
\varepsilon_{12} \\
\varepsilon_{23} \\
\varepsilon_{13}
\end{array}\right\}
$$

que em notação tensorial e indicial assume a forma compacta:

$$
\sigma_{i j}=C_{i j k l} \varepsilon_{k l} \quad i, j, k, l=1,2,3 .
$$

onde $C_{i j k l}$ é um tensor de quarta ordem que contém todos os parâmetros necessários para caracterizar qualquer tipo material, com um total de 81 componentes. Contudo, várias considerações podem ser aplicadas, tais como a simetria dos tensores de tensão e deformação e o conceito da energia de deformação. Desta maneira, considerando também que se trata de um material homogêneo e isotrópico, chega-se à seguinte relação:

$$
\sigma_{i j}=\lambda \varepsilon_{k k} \delta_{i j}+2 \mu \varepsilon_{i j}
$$

onde $\delta_{i j}$ é o delta de Kronecker, definido por:

$$
\delta_{i j}=\left\{\begin{array}{l}
\text { para } i=j, 1 \\
\text { para } i \neq j, 0
\end{array}\right.
$$

$\lambda$ e $\mu$ são conhecidas como as constantes de Lamé e são relacionadas aos módulos de Elasticidade $E$, coeficiente ou razão de Poisson $\nu$ e módulo de cisalhamento $G$ por:

$$
\begin{array}{r}
\lambda=\frac{E \nu}{(1+\nu)(1-2 \nu)} \\
\mu=G=\frac{E}{2(1+\nu)}
\end{array}
$$

A equação (2.29) é a lei de Hooke generalizada para sólidos elásticos que são formados de um material isotrópico e linear. A forma inversa desta relação, na qual as deformações são obtidas a partir das tensões, é dada por: 


$$
\varepsilon_{i j}=\frac{1}{2 \mu}\left(\sigma_{i j}-\frac{\lambda}{3 \lambda+2 \mu} \sigma_{k k} \delta_{i j}\right)
$$

As equações governantes de elasticidade podem ser obtidas substituindo as relações tensão deformação da equação (2.28) nas equações de equilíbrio (2.24). Esse resultado é usado nas relações entre deformações e deslocamentos (2.6), para resultar em:

$$
\mu u_{i, j j}+(\mu+\lambda) u_{j, j i}+b_{i}=0
$$

que são conhecidas como as equações de Navier para os deslocamentos.

\subsubsection{Condição de Tensão Plana}

A condição de tensão plana pode ser assumida quando está sendo analisada uma placa fina que não sofre carregamentos na direção de sua espessura. As tensões $\sigma_{11}$, $\sigma_{22}$ e $\sigma_{12}$ são então assumidas como independentes da coordenada $x_{3}$. A tensão $\sigma_{33}$ é nula e a deformação $\varepsilon_{33}$ é dada por:

$$
\varepsilon_{33}=\frac{-\nu}{1-\nu}\left(\varepsilon_{11}+\varepsilon_{22}\right)
$$

As relações tensão deformação ficam então:

$$
\begin{aligned}
\varepsilon_{11} & =\frac{1}{E}\left(\sigma_{11}-\nu \sigma_{22}\right) \\
\varepsilon_{22} & =\frac{1}{E}\left(\sigma_{22}-\nu \sigma_{11}\right) \\
\varepsilon_{12} & =\frac{1+\nu}{E} \sigma_{12}
\end{aligned}
$$

\subsection{Teoria de placas deformáveis por cisalhamento}

A placa é um elemento estrutural com formato plano cujas dimensões são grandes quando comparadas a sua espessura e sujeita a um carregamento transversal que causa deformações de flexão em conjunto com alongamentos. Na maioria dos casos, a espessura não é maior que um décimo da menor dimensão no plano. Por conta disso, é possível evitar o uso de formulações tridimensionais para a sua análise. Do ponto de vista computacional isso resulta em menores custos uma vez que a ordem dos elementos é reduzida em um. A dita teoria de placas espessas, é uma extensão da teoria de Kirchhoff-Love [6], ou teoria de placas finas, que leva em consideração as deformações cisalhantes através da espessura da placa. Por isso, também ficou conhecida como teoria de placas deformáveis por cisalhamento [2]. 


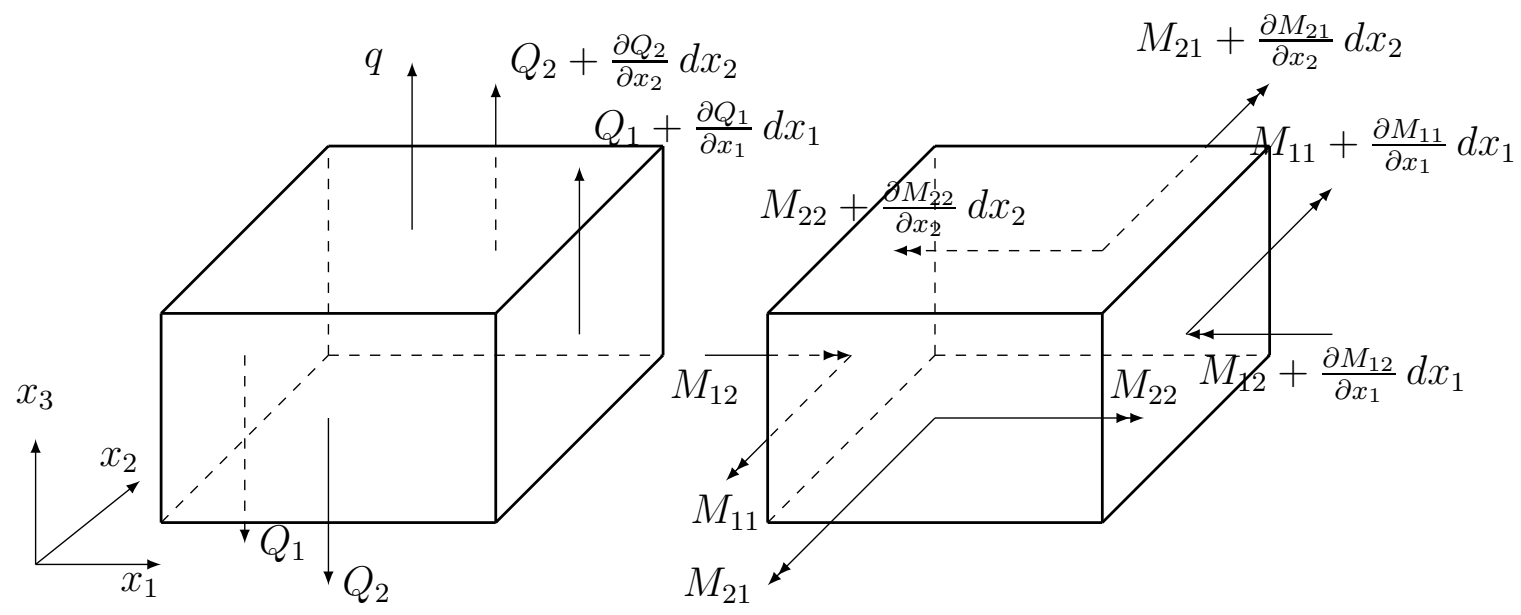

Figura 2.6: Situação de equilíbrio para um elemento de placa. Os carregamentos foram separados em duas imagens para facilitar a visualização.

As teorias de placas espessas foram desenvolvidas assumindo uma forma para o campo de deslocamentos ou para o campo de tensões como uma combinação linear de funções desconhecidas e a coordenada da espessura. As duas principais teorias de placas espessas que incorporam a deformação transversal cisalhante são: a teoria de Mindlin [8] e a de Reissner [7], que foram propostas de forma independente. É possível encontrar na literatura referências à teoria de Mindlin-Reissner categorizada como teoria de placas com deformação cisalhante de primeira ordem. As duas teorias não são iguais apesar de possuírem uma estreita relação. A principal distinção entre as duas está no modo como foram derivadas. A teoria de Reissner foi derivada do princípio variacional da energia complementar de deformação, com a suposição de variação linear de tensão flexural e distribuição parabólica de tensão cisalhante. A teoria de Mindlin, por sua vez, foi formulada assumindo uma variação linear de deslocamento através da espessura da placa, mantendo a deformação na direção transversal nula. As diferenças exatas e suas consequências foram apreciadas analiticamente e numericamente em [36]. Neste Trabalho a teoria de Reissner será utilizada.

\subsubsection{Equações de Equilíbrio}

As equações de equilíbrio podem ser obtidas de duas maneiras, utilizando o princípio de deslocamentos virtuais ou o equilíbrio de um elemento de placa típico como aquele mostrado na Figura 2.6.

Este elemento tem dimensões $d x_{1} \times d x_{2} \times h$ e está sujeito a um carregamento $q$ por unidade de área. De acordo com o descrito na Figura 2.6, o equilíbrio de momentos ao redor do eixo $x_{1}$ é dado por: 


$$
\begin{gathered}
M_{22} d x_{1}-\left(M_{22}+\frac{\partial M_{22}}{\partial x_{2}} d x_{2}\right) d x_{1}+M_{12} d x_{2}-\left(M_{12}+\frac{\partial M_{12}}{\partial x_{1}} d x_{1}\right) d x_{2}- \\
Q_{1} d x_{2} \frac{d x_{2}}{2}\left(Q_{1}+\frac{\partial Q_{1}}{\partial x_{1}} d x_{1}\right) d x_{2} \frac{d x_{2}}{2}+ \\
\left(Q_{2}+\frac{\partial Q_{2}}{\partial x_{2}} d x_{2}\right) d x_{1} d x_{2}+q d x_{1} d x_{2} \frac{d x_{2}}{2}=0
\end{gathered}
$$

Simplificando esta expressão e eliminando as multiplicações de termos infinitesimais, obtém-se:

$$
\frac{\partial M_{22}}{\partial x_{2}}+\frac{\partial M_{12}}{\partial x_{1}}-Q_{2}=0
$$

Procedendo da mesma maneira, considerando o equilíbrio de momentos ao redor do eixo $x_{2}$ e realizando as mesmas simplificações, chega-se a:

$$
\frac{\partial M_{11}}{\partial x_{1}}+\frac{\partial M_{21}}{\partial x_{2}}-Q_{1}=0
$$

Considerando agora o equilíbrio de forças na direção do eixo $x_{3}$, obtêm-se:

$$
-Q_{1} d x_{1} d x_{2}-Q_{2} d x_{1} d x_{2}+\left(Q_{1}+\frac{\partial Q_{1}}{\partial x_{1}}\right) d x_{1} d x_{2}+\left(Q_{2}+\frac{\partial Q_{2}}{\partial x_{2}}\right) d x_{2} d x_{1}+q d x_{1} d x_{2}=0
$$

que simplificada resulta em:

$$
\frac{\partial Q_{1}}{\partial x_{1}}+\frac{\partial Q_{2}}{\partial x_{2}}+q=0
$$

As igualdades (2.40), (2.41) e (2.43) representam as equações governantes em sua forma diferencial e quando escritas em notação indicial resultam em:

$$
\begin{aligned}
& M_{\alpha \beta, \beta}-Q_{\alpha}=0 \\
& Q_{\alpha, \alpha}+q=0 \quad \alpha, \beta=1,2 .
\end{aligned}
$$

\subsubsection{Relações tensões resultantes - deformações}

Antes de deduzir as relações entre tensões resultantes e deformação, algumas equações úteis serão apresentadas. Para um material isotrópico linear a relação tensão deformação é dado pela equação (2.45) [2]: 


$$
\left\{\begin{array}{l}
\sigma_{11} \\
\sigma_{22} \\
\sigma_{12} \\
\sigma_{13} \\
\sigma_{23}
\end{array}\right\}=\left[\begin{array}{ccccc}
\frac{E}{1-\nu^{2}} & \frac{\nu E}{1-\nu^{2}} & 0 & 0 & 0 \\
\frac{\nu E}{1-\nu^{2}} & \frac{E}{1-\nu^{2}} & 0 & 0 & 0 \\
0 & 0 & G & 0 & 0 \\
0 & 0 & 0 & G & 0 \\
0 & 0 & 0 & 0 & G
\end{array}\right]\left\{\begin{array}{l}
\varepsilon_{11} \\
\varepsilon_{22} \\
\varepsilon_{12} \\
\varepsilon_{13} \\
\varepsilon_{23}
\end{array}\right\}
$$

onde $E$ é o módulo de elasticidade e $\nu$ é a razão de poisson. O módulo de cisalhamento pode ser obtido pela expressão $G=E /(2(1+\nu))$. A relação entre tensões resultantes e os momentos fletores e forças cisalhantes são definidas como:

$$
\begin{aligned}
M_{\alpha \beta} & =\int_{-h / 2}^{h / 2} x_{3} \sigma_{\alpha \beta} d x_{3} \\
Q_{\alpha} & =\int_{-h / 2}^{h / 2} \sigma_{\alpha 3} d x_{3}
\end{aligned}
$$

A partir destas expressões as relações entre tensões resultantes e deformações são obtidas usando o teorema do trabalho mínimo de Castigliano, definindo a energia complementar dada pela equação (2.48) [13]:

$$
\Pi_{c}=\Pi_{d}-\Pi_{c}
$$

onde $\Pi_{d}$ é a energia de deformação e $\Pi_{c}$ caracteriza o trabalho realizado ao longo do contorno, estes termos são dados por (2.49) e (2.50):

$$
\begin{aligned}
\Pi_{d} & =\frac{1}{2} \int_{\Omega}\left\{\frac{M_{\alpha \beta} M_{\alpha \beta}(1+\nu)-\nu M_{\alpha \alpha} M_{\theta \theta}}{\left(1-\nu^{2}\right) D}-\frac{2}{C_{n}} q M_{\alpha \alpha}+\frac{1}{C_{s}} Q_{\alpha} Q_{\alpha}\right\} d \Omega \\
\Pi_{c} & =\int_{\Gamma}\left(M_{n} w_{n}+M_{t} w_{t}+Q_{n} w_{3}\right) d \Gamma
\end{aligned}
$$

nas quais

$$
\begin{aligned}
M_{n} & =M_{\alpha \beta} n_{\beta} n_{\alpha} \\
M_{t} & =M_{\alpha \beta} n_{\beta} t_{\alpha} \\
Q_{n} & =p_{3} \\
w_{n} & =w_{\gamma} n_{\gamma} \\
w_{t} & =w_{\gamma} t_{\gamma}
\end{aligned}
$$

onde os termos $M_{n}, M_{t}, Q_{n}, u_{n}, u_{t}$ são, respectivamente, o momento em torno da direção normal, o momento em torno da direção tangencial, o cisalhamento normal (Figura 


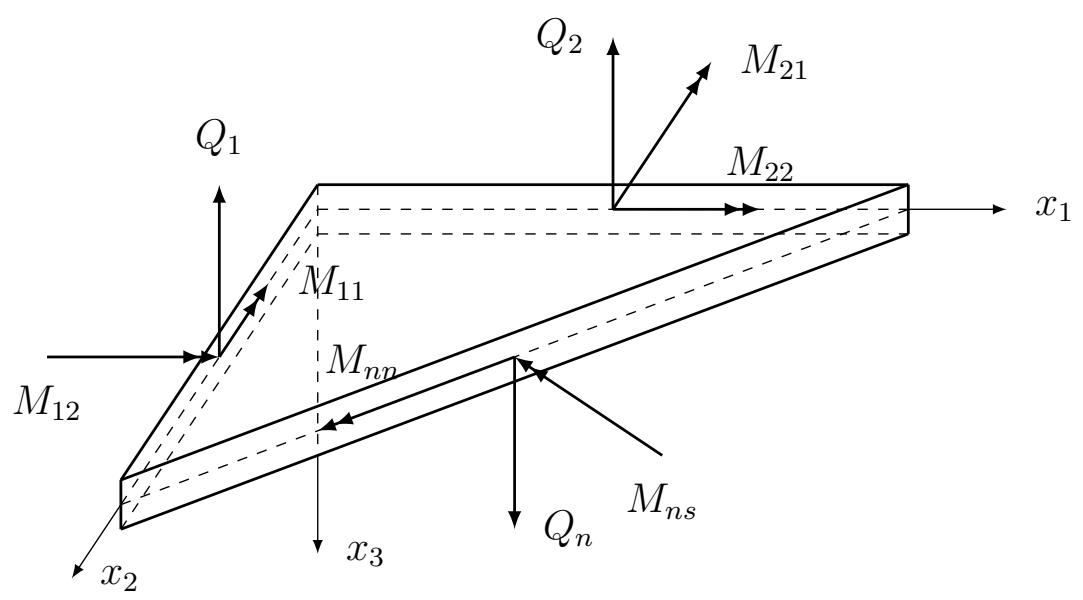

Figura 2.7: Ilustração mostrando as variáveis do problema de placa espessa em um ponto do contorno [2].

2.7), o deslocamento normal e tangencial em um ponto do contorno. Os vetores $n_{\alpha} \mathrm{e}$ $t_{\alpha}$ são, respectivamente, os vetores normais e tangenciais para um ponto no contorno. As constantes $D, C_{n}$ e $C_{s}$, na equação (2.52) dependem diretamente das propriedades do material.

Agora, de maneira a encontrar um mínimo da energia complementar para as equações governantes (2.44) devemos multiplicá-las por uma função ponderadora $w_{i} \mathrm{e}$ integrá-las ao longo do domínio. De modo a encontrar o mínimo, devemos então escrever o variacional dessa expressão e então igualá-la a zero, como mostrado em (2.52) [13]:

$$
\delta\left[\int_{\Omega}\left\{\left(M_{\alpha \beta, \beta}-Q_{\alpha}\right) w_{\alpha}+\left(Q_{\alpha, \alpha}+q\right) w_{3}\right\} d \Omega\right]=0
$$

onde $\delta$ é o operador variacional [37]. Utilizando a integração por partes para eliminar os variacionais de derivadas, resulta em:

$$
\begin{array}{r}
\int_{\Omega}\left\{\left[\frac{M_{\alpha \beta}(1+\nu)-\nu M_{\theta \theta} \delta_{\alpha \beta}}{\left(1-\nu^{2}\right) D}-\frac{q}{C_{n}} \delta_{\alpha \beta}-w_{\alpha, \beta}\right] \delta\left[M_{\alpha \beta}\right]\right. \\
\left.+\left[\frac{Q_{\alpha}}{C_{s}}-w_{3, \alpha}-w_{\alpha}\right] \delta\left[Q_{\alpha}\right]\right\} d \Omega=0
\end{array}
$$

implicando que para a igualdade se tornar verdadeira:

$$
\begin{aligned}
\frac{M_{\alpha \beta}(1+\nu)-\nu M_{\theta \theta} \delta_{\alpha \beta}}{\left(1-\nu^{2}\right) D}-\frac{q}{C_{n}} \delta_{\alpha \beta}-w_{\alpha, \beta} & =0 \\
\frac{Q_{\alpha}}{C_{s}}-w_{3, \alpha}-w_{\alpha} & =0
\end{aligned}
$$

onde rearranjando os termos e inserindo as seguintes substituições: 


$$
\begin{aligned}
\chi_{\alpha \beta} & =\frac{1}{2}\left(w_{\alpha, \beta}+w_{\beta, \alpha}\right) \\
\psi_{\alpha} & =w_{\alpha}+w_{3, \alpha}
\end{aligned}
$$

obtêm-se:

$$
\begin{aligned}
M_{\alpha \beta} & =D \frac{1-\nu}{2}\left(2 \chi_{\alpha \beta}+\frac{2 \nu}{1-\nu} \chi_{\gamma \gamma} \delta_{\alpha \beta}\right)+D \frac{1+\nu}{C_{n}} q \delta_{\alpha \beta} \\
Q_{\alpha} & =C_{s} \psi_{\alpha}
\end{aligned}
$$

na qual $\delta_{\alpha \beta}$ é o delta de kronecker, $\chi_{\alpha \beta}$ é a deformação de flexão e $\psi_{\alpha}$ é a deformação cisalhante. A equação (2.57) é a lei de Hooke generalizada e representa a relação entre tensões resultantes e deformações. As constantes apresentadas nestas equações possuem valores:

$$
\begin{aligned}
D & =\frac{E h^{3}}{12\left(1-\nu^{2}\right)} \\
C_{n} & =\frac{5 E h}{6 \nu} \\
C_{s} & =\frac{5 G h}{6}
\end{aligned}
$$

Substituindo os valores das constantes em (2.58) na equação (2.57), resulta em:

$$
\begin{aligned}
M_{\alpha \beta} & =D \frac{1-\nu}{2}\left(w_{\alpha, \beta}+w_{\beta, \alpha}+\frac{2 \nu}{1-\nu} w_{\gamma, \gamma} \delta_{\alpha \beta}\right)+\frac{q \nu}{(1-\nu) \lambda^{2}} \delta_{\alpha \beta} \\
Q_{\alpha} & =D \frac{1-\nu}{2} \lambda^{2}\left(w_{\alpha}+w_{3, \alpha}\right)
\end{aligned}
$$

que são as relações entre resultantes de tensão e deslocamentos generalizados, onde $\lambda=\sqrt{10} / h$ é o fator de cisalhamento.

As equações de Navier generalizadas podem ser obtidas aplicando a equação (2.59) nas equações governantes (2.44):

$$
L_{i j} w_{j}+b_{i}=0
$$

na qual: 


$$
\begin{aligned}
L_{\alpha \beta} & =D \frac{1-\nu}{2}\left[\left(\nabla^{2}-\lambda^{2}\right) \delta_{\alpha \beta}+\frac{1+\nu}{1-\nu} \partial_{\alpha} \partial_{\beta}\right] \\
L_{\alpha 3} & =-D\left(\frac{1-\nu}{2}\right) \lambda^{2} \partial_{\alpha} \\
L_{3 \beta} & =D\left(\frac{1-\nu}{2}\right) \lambda^{2} \partial_{\beta} \\
L_{33} & =D\left(\frac{1-\nu}{2}\right) \lambda^{2} \nabla^{2}
\end{aligned}
$$

onde $L_{i j}$ é o operador de Navier generalizado, $\nabla^{2}$ é o laplaciano bidimensional e $b_{i}$ são as forças de corpo generalizadas, dadas por:

$$
\begin{aligned}
b_{\alpha} & =\frac{\nu}{(1-\nu) \lambda^{2}} q_{, \alpha} \\
b_{3} & =q
\end{aligned}
$$

\subsubsection{Tipos de condições de contorno}

A formulação de placas possui variáveis relacionadas a duas rotações em torno dos eixos $x_{1}$ e $x_{2}$ e relacionadas a uma deflexão em $x_{3}$. Assim, quando da resolução do problema, é necessário definir condições de contorno adequadas e que possibilitem a sua resolução. Nesta seção, os termos $w_{\alpha}$ se referem às rotações e $w_{3}$ ao deslocamento na direção $x_{3}$. De maneira geral podemos resumir as possibilidades de restrições como:

- Contorno engastado, onde: $w_{t}=0, w_{n}=0$ e $u_{3}=0$.

- Contorno simplesmente apoiado, onde: $w_{t}=0, w_{3}=0$ e $M_{n}=0$.

- Contorno guiado, onde: $w_{n}=0, w_{t}=0$ e $p_{3}=0$.

- Contorno livre, onde: $M_{n}=0, M_{t}=0$ e $p_{3}=0$. 


\section{MÉTODO DOS ELEMENTOS DE CONTORNO}

\subsection{Formulação do MEC para Elasticidade}

Nesta seção a formulação do método dos elementos de contorno para elasticidade será desenvolvida. Os itens a serem abordados englobam a obtenção da equação integral de contorno (EIC), a obtenção da solução fundamental e a especialização da EIC para um ponto qualquer, dentro do domínio, exatamente no contorno ou externo a ele.

Entre as várias possibilidades de obtenção das equações integrais de contorno para elasticidade, podemos utilizar o teorema da reciprocidade de Betti. A formulação é obtida a partir deste teorema, considerando dois estados equilibrados, um no domínio do problema e o outro naquele conhecido como espaço fundamental, com variáveis $\left(u_{i}, t_{i}, b_{i}\right)$ e $\left(u_{i}^{*}, t_{i}^{*}, b_{i}^{*}\right)$, respectivamente, onde $u_{i}$ e $u_{i}^{*}$ são os deslocamentos, $t_{i}$ e $t_{i}^{*}$ são as forças de superfície, e $b_{i}$ e $b_{i}^{*}$ são as forças de corpo. Ao longo deste capítulo os índices serão alterados de letras romanas para gregas assim que a formulação for especificada para problemas bidimensionais. A derivação descrita nesta seção segue aquela desenvolvida em [12].

O processo se inicia integrando a equação de equilíbrio (2.24) ao longo do domínio, representado por $\Omega$, e ponderando-a por $u_{i}^{*}$. Dessa maneira, chega-se à seguinte expressão:

$$
\int_{\Omega}\left(\sigma_{i j, j}+b_{i}\right) u_{i}^{*} d \Omega=0 \quad i, j=1,2,3 .
$$

As tensões $\sigma_{i j}$, as forças de corpo $b_{i}$ e deslocamentos $u_{i}$ são funções da posição $\left(x_{1}, x_{2}, x_{3}\right)$ no domínio $\Omega$. A função ponderadora $u_{i}^{*}$ foi escolhida arbitrariamente e ainda não está definida. Considerando a integral do termo $\sigma_{i j, j} u_{i}^{*}$, usando a regra do produto da derivação,

$$
\begin{aligned}
h(x) & =g(x) f(x) \\
h^{\prime}(x) & =g^{\prime}(x) f(x)+g(x) f^{\prime}(x) \\
g^{\prime}(x) f(x) & =h^{\prime}(x)-g(x) f^{\prime}(x)
\end{aligned}
$$

e fazendo $g^{\prime}=\sigma_{i j, j}$ e $f=u_{i}^{*}$, é possível reescrevê-la como:

$$
\int_{\Omega} \sigma_{i j, j} u_{i}^{*} d \Omega=\int_{\Omega}\left(\sigma_{i j} u_{i}^{*}\right)_{, j} d \Omega-\int_{\Omega} \sigma_{i j} \varepsilon_{i j}^{*} d \Omega
$$

onde o segundo termo do lado direito da equação acima foi obtido considerando que: 


$$
\begin{aligned}
\sigma_{i j} u_{i, j}^{*} & =\sigma_{i j} \frac{1}{2}\left(u_{i, j}^{*}+u_{i, j}^{*}\right) \\
& =\sigma_{i j} \varepsilon_{i j}^{*}
\end{aligned}
$$

na qual a simetria de $\sigma_{i j}$ e a propriedade $(A B)^{T}=B^{T} A^{T}$ foram empregadas. Usando agora o teorema da divergência que estabelece:

$$
\int_{\Omega} \Phi_{i, i} d \Omega=\int_{\Gamma} \Phi_{i} n_{i} d \Gamma
$$

onde $\Gamma$ é o contorno de um domínio $\Omega$. Levando em consideração a relação entre forças de superfície e tensões mostrada na equação (2.23), o primeiro termo do lado direito da equação (3.2) fica:

$$
\int_{\Omega}\left(\sigma_{i j} u_{i}^{*}\right)_{, j} d \Omega=\int_{\Gamma} \sigma_{i j} n_{j} u_{i}^{*} d \Gamma=\int_{\Gamma} t_{i} u_{i}^{*} d \Gamma
$$

assim, a equação (3.2) pode ser reescrita como:

$$
\int_{\Omega} \sigma_{i j, j} u_{i}^{*} d \Omega=\int_{\Gamma} t_{i} u_{i}^{*} d \Gamma-\int_{\Omega} \sigma_{i j} \varepsilon_{i j}^{*} d \Omega
$$

Substituindo agora (3.6) em (3.1), obtém-se:

$$
\int_{\Omega} b_{i} u_{i}^{*} d \Omega+\int_{\Gamma} t_{i} u_{i}^{*} d \Gamma=\int_{\Omega} \sigma_{i j} \varepsilon_{i j}^{*} d \Omega
$$

Agora, deve-se mostrar que:

$$
\int_{\Omega} \sigma_{i j} \varepsilon_{i j}^{*} d \Omega=\int_{\Omega} \sigma_{i j}^{*} \varepsilon_{i j} d \Omega
$$

e para isso leva-se em consideração que, $a_{m m}=\delta_{i j} a_{i j}$ e a relação entre tensões e deformações para um corpo homogêneo e isotrópico dada pela equação (2.29), repetida aqui por conveniência.

$$
\sigma_{i j}=\lambda \varepsilon_{k k} \delta_{i j}+2 \mu \varepsilon_{i j}
$$

A sequência de manipulações partindo do termo no lado esquerdo da equação (3.8) fica: 


$$
\begin{aligned}
\int_{\Omega} \sigma_{i j} \varepsilon_{i j}^{*} d \Omega & =\int_{\Omega} \lambda \varepsilon_{k k} \delta_{i j} \varepsilon_{i j}^{*}+2 \mu \varepsilon_{i j} \varepsilon_{i j}^{*} d \Omega \\
& =\int_{\Omega} \lambda \delta_{i j} \varepsilon_{i j} \delta_{i j} \varepsilon_{i j}^{*}+2 \mu \varepsilon_{i j} \varepsilon_{i j}^{*} d \Omega \\
& =\int_{\Omega}\left(\lambda \delta_{i j} \delta_{i j} \varepsilon_{i j}^{*}+2 \mu \varepsilon_{i j}^{*}\right) \varepsilon_{i j} d \Omega \\
& =\int_{\Omega}\left(\lambda \delta_{i j} \varepsilon_{k k}^{*}+2 \mu \varepsilon_{i j}^{*}\right) \varepsilon_{i j} d \Omega \\
& =\int_{\Omega} \sigma_{i j}^{*} \varepsilon_{i j} d \Omega
\end{aligned}
$$

provando a igualdade (3.8). Usando as equações (3.7) e (3.8), pode-se afirmar que a seguinte relação é verdadeira:

$$
\int_{\Omega} b_{i} u_{i}^{*} d \Omega+\int_{\Gamma} t_{i} u_{i}^{*} d \Gamma=\int_{\Omega} b_{i}^{*} u_{i} d \Omega+\int_{\Gamma} t_{i}^{*} u_{i} d \Gamma
$$

conhecida como o teorema do trabalho recíproco de Betti.

\subsubsection{Equação integral de contorno para elasticidade}

A partir do desenvolvimento matemático desenvolvido na seção anterior, que resultou na expressão (3.10), deve-se fazer agora com que o termo $b_{i}^{*}$ corresponda a uma carga pontual de módulo unitário em um domínio de extensão infinita, que pode ser representada pela função delta de $\operatorname{Dirac} \delta\left(\mathbf{X}-\mathbf{X}^{\prime}\right)$ como:

$$
b_{i}^{*}=\delta\left(\mathbf{X}-\mathbf{X}^{\prime}\right) e_{i}
$$

onde a componente do vetor unitário $e_{i}$ corresponde a uma carga de módulo unitário na direção positiva $x_{i}$ aplicada em $\mathbf{X}^{\prime}$. A função delta de Dirac é utilizada em aplicações de engenharia em casos em que excitações são idealizadas como ocorrendo em um ponto. Forças concentradas em problemas elásticos e fontes geradoras pontuais em análises de transferência de calor são dois exemplos comuns de seu emprego. Apesar de sua utilidade matemática, a contra parte real de uma carga concentrada não existe, uma vez que uma imposição de carregamento desta maneira iria gerar tensões localizadas altíssimas que não seriam suportadas pelo material.

Uma propriedade chave do delta de Dirac para as formulações do método dos elementos de contorno, envolve a identidade:

$$
\int_{\Omega} f(\mathbf{X}) \delta\left(\mathbf{X}-\mathbf{X}^{\prime}\right) d \Omega=f\left(\mathbf{X}^{\prime}\right)
$$

conhecida como propriedade de seleção [38]. Utilizando-a é possível escrever que: 


$$
\int_{\Omega} b_{i}^{*} u_{i} d \Omega=\int_{\Omega} \delta\left(\mathbf{X}-\mathbf{X}^{\prime}\right) e_{i} u_{i} d \Omega=u_{i}\left(\mathbf{X}^{\prime}\right) e_{i}
$$

A aplicação desta carga unitária na equação de Navier (2.34), torna possível a definição dos campos correspondentes de deslocamentos e forças de superfície no espaço fundamental, escritos como:

$$
\begin{aligned}
u_{i}^{*} & =U_{i j}\left(\mathbf{X}^{\prime}, \mathbf{X}\right) e_{j} \\
t_{i}^{*} & =T_{i j}\left(\mathbf{X}^{\prime}, \mathbf{X}\right) e_{j}
\end{aligned}
$$

Substituindo as soluções obtidas acima e considerando (3.13), a equação (3.10) se torna:

$$
\begin{aligned}
u_{i}\left(\mathbf{X}^{\prime}\right)= & \int_{\Gamma} U_{i j}\left(\mathbf{X}^{\prime}, \mathbf{X}\right) t_{j}(\mathbf{X}) d \Gamma(\mathbf{x}) \\
& -\int_{\Gamma} T_{i j}\left(\mathbf{X}^{\prime}, \mathbf{X}\right) u_{j}(\mathbf{X}) d \Gamma(\mathbf{x})+\int_{\Omega} U_{i j}\left(\mathbf{X}^{\prime}, \mathbf{X}\right) b_{j}(\mathbf{X}) d \Omega(\mathbf{X})
\end{aligned}
$$

Essa expressão é conhecida como identidade de Somigliana ou equação integral de contorno para os deslocamentos. Na forma matricial e para duas dimensões a equação (3.16) fica:

$$
\begin{aligned}
\left\{\begin{array}{l}
u_{1} \\
u_{2}
\end{array}\right\}= & \int_{\Gamma}\left[\begin{array}{ll}
U_{11} & U_{12} \\
U_{21} & U_{22}
\end{array}\right]\left\{\begin{array}{l}
t_{1} \\
t_{2}
\end{array}\right\} d \Gamma-\int_{\Gamma}\left[\begin{array}{ll}
T_{11} & T_{12} \\
T_{21} & T_{22}
\end{array}\right]\left\{\begin{array}{l}
u_{1} \\
u_{2}
\end{array}\right\} d \Gamma+ \\
& \int_{\Omega}\left[\begin{array}{ll}
U_{11} & U_{12} \\
U_{21} & U_{22}
\end{array}\right]\left\{\begin{array}{l}
b_{1} \\
b_{2}
\end{array}\right\} d \Omega
\end{aligned}
$$

A identidade de Somigliana para tensões pode ser obtida a partir de (3.16) derivandoa em relação ao ponto fonte $\mathbf{X}^{\prime}$, o que resulta em:

$$
\begin{aligned}
u_{i, k}\left(\mathbf{X}^{\prime}\right) & =\int_{\Gamma} U_{i j, k}\left(\mathbf{X}^{\prime}, \mathbf{X}\right) t_{j}(\mathbf{X}) d \Gamma(\mathbf{x}) \\
& -\int_{\Gamma} T_{i j, k}\left(\mathbf{X}^{\prime}, \mathbf{X}\right) u_{j}(\mathbf{X}) d \Gamma(\mathbf{x})+\int_{\Omega} U_{i j, k}\left(\mathbf{X}^{\prime}, \mathbf{X}\right) b_{j}(\mathbf{X}) d \Omega(\mathbf{X})
\end{aligned}
$$

onde $U_{i j, k}$ e $T_{i j, k}$ são as derivadas das soluções fundamentais. Substituindo agora (3.18) na lei de Hooke para materiais isotrópicos e homogêneos (2.29) chega-se a:

$$
\begin{aligned}
\sigma_{i j}\left(\mathbf{X}^{\prime}\right) & =\int_{\Gamma} D_{k i j}\left(\mathbf{X}^{\prime}, \mathbf{x}\right) t_{j}(\mathbf{x}) d \Gamma(\mathbf{x}) \\
& -\int_{\Gamma} S_{k i j}\left(\mathbf{X}^{\prime}, \mathbf{x}\right) u_{j}(\mathbf{x}) d \Gamma(\mathbf{x})+\int_{\Omega} D_{k i j}\left(\mathbf{X}^{\prime}, \mathbf{X}\right) b_{j}(\mathbf{X}) d \Omega(\mathbf{X})
\end{aligned}
$$


na qual os termos $D_{k i j}$ e $S_{k i j}$ são obtidos a partir das derivadas das soluções fundamentais $U_{i j, k}$ e $T_{i j, k}$ e da aplicação da Lei de Hooke.

\subsubsection{Soluções Fundamentais}

A solução fundamental tem papel crucial para as formulações do MEC. Sua existência faz com que seja possível aplicar a técnica e a sua definição está relacionada com o desenvolvimento matemático que resulta na identidade de Somigliana. Na seção anterior considerou-se que o termo $b_{i}^{*}$ fosse igualado a uma carga pontual na direção de $\mathbf{e}_{i}$ aplicada em um domínio infinito. Substituindo essa definição, representada pela equação (3.11), na equação de Navier (2.34), tem-se que:

$$
\mu u_{i, j j}^{*}+(\mu+\lambda) u_{j, j i}^{*}+\delta\left(\mathbf{X}-\mathbf{X}^{\prime}\right) e_{i}=0
$$

onde os termos de deslocamentos são aqueles do espaço fundamental, correspondentes à carga unitária pontual aplicada. A solução desta expressão dará origem à equação conhecida como solução fundamental para a elasticidade. De maneira geral, um resultado obtido a partir da equação governante de um problema com carregamento devido a uma força pontual e unitária dará origem à uma solução fundamental aplicável às formulações do MEC.

Entre os vários métodos de solução deste problema, será aqui apresentado o método que utiliza o vetor de Galerkin $(\mathbf{G})$, uma estratégia que visa simplificar a equação do problema através da seguinte substituição:

$$
u_{i}^{*}=G_{i, k k}-\frac{1}{2(1-\nu)} G_{k, i k}
$$

Substituindo (3.21) em (3.20) resulta em:

$$
\mu G_{i, k k j j}-\frac{\mu}{2(1-\nu)} G_{k, i k j j}+\frac{\mu}{1-\nu}\left(G_{j, k k i j}-\frac{1}{2(1-\nu)} G_{k, j k i j}\right)+\delta\left(\mathbf{X}-\mathbf{X}^{\prime}\right) e_{i}=0
$$

que pode ser convenientemente simplificada para:

$$
\mu G_{i, k k j j}+\delta\left(\mathbf{X}-\mathbf{X}^{\prime}\right) e_{i}=0
$$

Essa equação também pode ser escrita na forma:

$$
\mu \nabla^{2}\left(\nabla^{2} G_{i}\right)+\delta\left(\mathbf{X}-\mathbf{X}^{\prime}\right) e_{i}=0
$$

Fazendo $F_{i}=\nabla^{2} G_{i}$, chega-se a:

$$
\mu \nabla^{2}\left(F_{i}\right)+\delta\left(\mathbf{X}-\mathbf{X}^{\prime}\right) e_{i}=0
$$




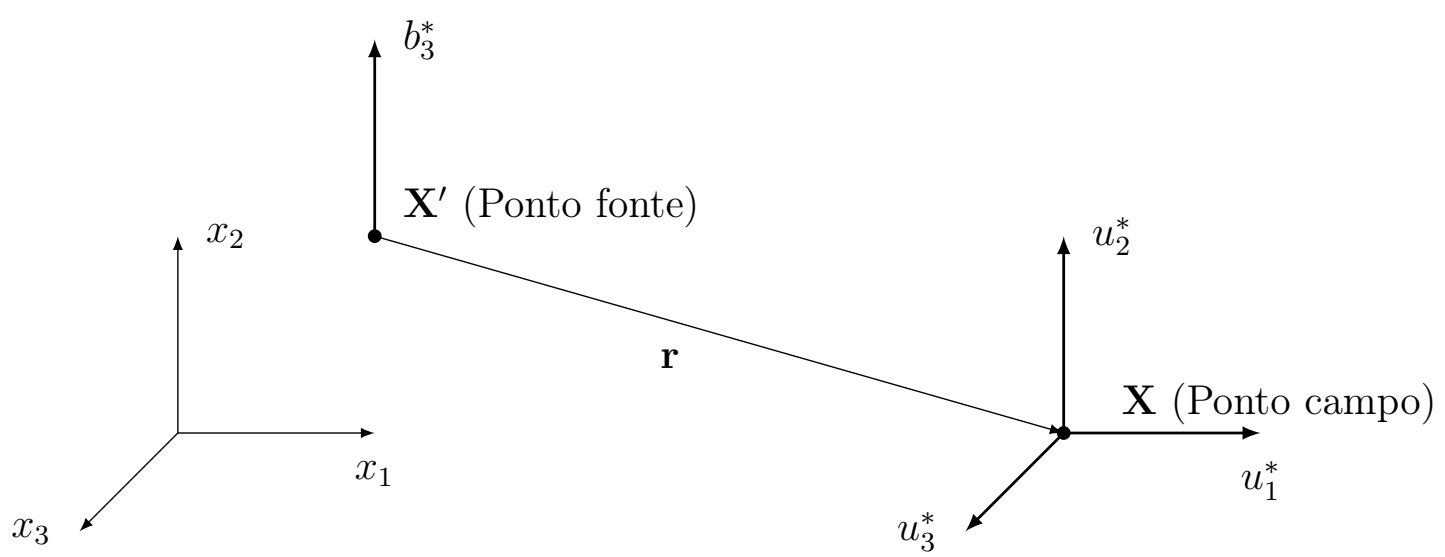

Figura 3.1: Ponto fonte e campo nos quais estão localizados uma carga pontual unitária e as deslocamento correspondente.

que possui resposta conhecida. Para o caso bidimensional a solução de (3.25) é:

$$
F_{\alpha}=-\frac{1}{2 \pi \mu} \ln (r) e_{\alpha} \quad \alpha=1,2 .
$$

com o correspondente vetor de Galerkin dado por:

$$
G_{\alpha}=-\frac{1}{8 \pi \mu} r^{2} \ln (r) e_{\alpha}
$$

Pode-se substituir este último resultado em (3.21) e, dessa maneira encontrar a solução fundamental para os deslocamentos:

$$
u_{\alpha}^{*}=\frac{1}{8 \pi \mu(1-\nu)}\left[(3-4 \nu) \ln \left(\frac{1}{r}\right) \delta_{\alpha \beta}+r_{, \alpha} r_{, \beta}\right] e_{\beta}
$$

e a partir de (3.14), chega-se finalmente à solução fundamental bidimensional para os deslocamentos:

$$
U_{\alpha \beta}^{*}\left(\mathbf{X}, \mathbf{X}^{\prime}\right)=\frac{1}{8 \pi \mu(1-\nu)}\left[(3-4 \nu) \ln \left(\frac{1}{r}\right) \delta_{\alpha \beta}+r_{, \alpha} r_{, \beta}\right]
$$

Esta solução representa o deslocamento na direção $\beta$ no ponto $\mathbf{X}$ causado pela aplicação de um carregamento pontual unitário na direção $\alpha$ no ponto $\mathbf{X}^{\prime}$. A relação entre estas grandezas varia com a distância $r$. Uma representação esquemática destas grandezas pode ser visualizado na Figura 3.1.

A expressão da solução fundamental de forças de superfície é obtida de (3.29), usando as relações entre deslocamentos e deformações, e na sequência entre tensões e deformações a partir da equação (2.23), o que resulta em: 


$$
\begin{aligned}
t_{i}^{*} & =\sigma_{i j}^{*} n_{j} \\
& =\left[\lambda \varepsilon_{k k}^{*} \delta_{i j}+2 \mu \varepsilon_{i j}^{*}\right] n_{j} \\
& =\left[\lambda \frac{1}{2}\left(u_{k, k}^{*}+u_{k, k}^{*}\right) \delta_{i j}+2 \mu \frac{1}{2}\left(u_{i, j}^{*}+u_{i, j}^{*}\right)\right] n_{j} \\
& =\left[\lambda u_{k, k}^{*} \delta_{i j}+\mu\left(u_{i, j}^{*}+u_{i, j}^{*}\right)\right] n_{j}
\end{aligned}
$$

Desta forma, a solução fundamental bidimensional para forças de superfície é:

$$
T_{\alpha \beta}^{*}=-\frac{1}{4 \pi(1-\nu) r}\left\{\frac{\partial r}{\partial n}\left[(1-2 \nu) \delta_{\alpha \beta}+2 r_{, \alpha} r_{, \beta}\right]+(1-2 \nu)\left(n_{\alpha} r_{, \beta}-n_{\beta} r_{, \alpha}\right)\right\}
$$

Os termos contidos nas equações integrais de contorno para deformação e tensões são obtidos diretamente das soluções fundamentais de deslocamento e forças de superfície e são dados por:

$$
\begin{aligned}
U_{\alpha \beta, \gamma}^{*}\left(\mathbf{X}^{\prime}, \mathbf{x}\right)= & \frac{1}{8 \pi \mu(1-\nu) r}\left[r_{, \alpha} \delta_{\beta \gamma}+r_{, \beta} \delta_{\alpha \gamma}-(3-4 \nu) r_{, \gamma} \delta_{\alpha \beta}+r_{, \alpha} r_{, \beta} r_{, \gamma}\right] \\
T_{\alpha \beta, \gamma}^{*}\left(\mathbf{X}^{\prime}, \mathbf{x}\right)= & -\frac{1}{4 \pi(1-\nu) r}\left\{\frac{\partial r}{\partial x_{\gamma} \partial n}\left[(1-2 \nu) \delta_{\alpha \beta}+2 r_{, \alpha} r_{, \beta}\right]+\right. \\
& \frac{\partial r}{\partial n}\left[(1-2 \nu) \delta_{\alpha \beta}+\frac{2}{r}\left(r_{, \alpha} \delta_{\beta \gamma}+r_{, \beta} \delta_{\alpha \gamma}-2 r_{, \alpha} r_{, \beta} r_{, \gamma}\right)\right] \\
& \left.+(1-2 \nu)\left[n_{\alpha}\left(\frac{\delta_{\beta \gamma}-r_{, \beta} r_{, \gamma}}{r}\right)-n_{\beta}\left(\frac{\delta_{\alpha \gamma}-r_{, \alpha} r_{, \gamma}}{r}\right)\right]\right\}
\end{aligned}
$$

onde

$$
\frac{\partial r}{\partial x_{\gamma} \partial n}=n_{\alpha}\left(\frac{\delta_{\alpha \gamma}-r_{, \alpha} r_{, \gamma}}{r}\right)
$$

e por:

$$
\begin{aligned}
D_{\gamma \alpha \beta}\left(\mathbf{X}^{\prime}, \mathbf{x}\right)= & \frac{1}{4 \pi(1-\nu) r}\left\{(1-2 \nu)\left(r_{\beta} \delta_{\gamma \alpha}-r_{\gamma} \delta_{\alpha \beta}+r_{\alpha} \delta_{\beta \gamma}\right)+2 r_{\alpha} r_{\beta} r_{\gamma}\right\} \\
S_{\gamma \alpha \beta}\left(\mathbf{X}^{\prime}, \mathbf{x}\right)= & \frac{\mu}{2 \pi r^{2}(1-\nu)}\left\{2 \frac{\partial r}{\partial n}\left[(1-2 \nu) \delta_{\alpha \beta} r_{, \gamma}+\left(r_{\beta} \delta_{\alpha \gamma}+r_{\alpha} \delta_{\beta \gamma}\right) \nu-4 r_{\alpha} r_{\beta} r_{\gamma}\right]\right. \\
& +2 \nu\left(n_{\alpha} r_{\alpha} r_{\gamma}+n_{\beta} r_{\alpha} r_{\gamma}\right)+(1-2 \nu)\left(2 n_{\gamma} r_{\alpha} r_{\beta}+n_{\beta} \delta_{\alpha \gamma}+n_{\alpha} \delta_{\beta \gamma}\right) \\
& \left.-(1-4 \nu) n_{\gamma} \delta_{\alpha \beta}\right\}
\end{aligned}
$$

\subsubsection{Equação integral de contorno de elasticidade especificada para pon- tos no contorno}

A equação integral de contorno dada pela expressão (3.16) é válida para o ponto fonte posicionado em qualquer localização do domínio. Considerações adicionais precisam ser feitas para que seja obtida uma equação válida para qualquer posição do ponto 
fonte. Os detalhes dessas considerações podem ser encontrados em várias referências $[38,12]$ mas consistem basicamente em posicionar um ponto no contorno do problema e envolvê-lo por uma extensão do domínio. Em seguida, emprega-se um processo limite no qual o tamanho dessa extensão diminui até que o novo contorno seja igual ao contorno original. Deste processo surgem termos livres que quando avaliados fazem com que a equação integral de contorno para problemas bidimensionais adquira a seguinte forma:

$$
\begin{aligned}
C_{\alpha \beta}\left(\mathbf{x}^{\prime}\right) u_{\alpha}\left(\mathbf{x}^{\prime}\right) & =\int_{\Gamma} U_{\alpha \beta}^{*}\left(\mathbf{x}^{\prime}, \mathbf{x}\right) t_{\beta}(\mathbf{x}) d \Gamma(\mathbf{x}) \\
& -f_{\Gamma} T_{\alpha \beta}^{*}\left(\mathbf{x}^{\prime}, \mathbf{x}\right) u_{\beta}(\mathbf{x}) d \Gamma(\mathbf{x})+\int_{\Omega} U_{\alpha \beta}^{*}\left(\mathbf{x}^{\prime}, \mathbf{X}\right) b_{\beta}(\mathbf{X}) d \Omega(\mathbf{X})
\end{aligned}
$$

na qual $\mathbf{x}$ representa um ponto no contorno e $f$ significa uma integração no sentido do valor principal de Cauchy. O termo livre para o caso bidimensional em um contorno suave é:

$$
C_{\alpha \beta}\left(\mathbf{x}^{\prime}\right)=\frac{1}{2} \delta_{\alpha \beta}
$$

\subsection{Formulação do MEC para Placas Espessas}

Como no caso da formulação do MEC para elasticidade, a formulação de placas é desenvolvida a partir de uma representação integral das equações diferenciais do problema. Assim, integrando as equações governantes (2.59) ao longo do domínio, representado por $\Omega$, e ponderando-a por $w_{i}^{*}$ chega-se a seguinte expressão:

$$
\int_{\Omega}\left[\left(M_{\alpha \beta, \beta}-Q_{\alpha}\right) w_{\alpha}^{*}+\left(Q_{\alpha, \alpha}+q\right) w_{3}^{*}\right] d \Omega=0
$$

na qual a função ponderadora $w_{i}^{*}$ foi escolhida arbitrariamente e ainda não está definida. Aplicando a segunda identidade de Green e utilizando as seguintes relações:

$$
\begin{aligned}
& p_{\alpha}=M_{\alpha \beta} n_{\beta} \\
& p_{3}=Q_{\alpha} n_{\alpha}
\end{aligned}
$$

resulta em:

$$
\int_{\Gamma} p_{j} w_{i}^{*} d \Gamma+\int_{\Omega} q w_{3}^{*} d \Omega+\int_{\Omega} Q_{\alpha}\left(w_{\alpha}^{*}+w_{3, \alpha}^{*}\right) d \Omega+\int_{\Omega} M_{\alpha \beta} w_{\alpha, \beta}^{*} d \Omega=0
$$

Substituindo os termos de resultantes de tensão, $M_{\alpha \beta}$ e $Q_{\alpha}$, por suas relações com os deslocamentos generalizados dadas pelas equações (2.59) e aplicando na sequência o 
segundo teorema de Green para o quarto termo do lado esquerdo da equação, resulta em:

$$
\begin{aligned}
& \int_{\Gamma} p_{j} w_{j}^{*} d \Gamma+\int_{\Omega} Q_{\alpha}^{*}\left(w_{\alpha}+w_{3, \alpha}\right) d \Omega \\
& -\int_{\Gamma} w_{\alpha, \beta}^{*}\left\{\frac{D(1-\nu)}{2}\left[w_{\alpha} n_{\beta}+w_{\beta} n_{\alpha}+\frac{2 \nu}{(1-\nu)} w_{\gamma} n_{\gamma} \delta_{\alpha \beta}\right]\right\} d \Gamma \\
& -\int_{\Gamma} \frac{D(1-\nu)}{2}\left[w_{\alpha} w_{\alpha, \beta \beta}^{*}+w_{\beta} w_{\alpha, \beta \alpha}^{*}+\frac{2 \nu}{(1-\nu)} w_{\gamma} w_{\alpha, \beta \gamma}^{*} \delta_{\alpha \beta}\right] d \Gamma \\
& +\int_{\Omega}\left[q w_{3}^{*}+\frac{\nu q}{(1-\nu) \lambda^{2}} \delta_{\alpha \beta} w_{\alpha, \beta}^{*}\right] d \Omega=0
\end{aligned}
$$

A segunda integral do lado esquerdo da equação (3.43) pode ser decomposta usando novamente a segunda identidade de Green e fazendo uso das relações em (3.41), de tal forma que se chega a:

$$
\begin{aligned}
\int_{\Omega} Q_{\alpha}^{*}\left(w_{\alpha}+w_{3, \alpha}\right) d \Omega & =\int_{\Omega} Q_{\alpha}^{*} w_{\alpha} d \Omega+\int_{\Omega} Q_{\alpha}^{*} w_{3, \alpha} d \Omega \\
& =\int_{\Omega} Q_{\alpha}^{*} w_{\alpha} d \Omega+\int_{\Gamma} Q_{\alpha}^{*} w_{3} n_{\alpha} d \Gamma-\int_{\Omega} Q_{\alpha, \alpha}^{*} w_{3} d \Omega \\
& =\int_{\Gamma} p_{3} w_{3} d \Gamma+\int_{\Omega}\left(Q_{\alpha}^{*} w_{\alpha}-Q_{\alpha, \alpha}^{*} w_{3}\right) d \Omega
\end{aligned}
$$

Substituindo agora (3.44) em (3.43) e rearranjando os termos, chega-se finalmente a:

$$
\begin{aligned}
& \int_{\Gamma}\left[w_{j}^{*} p_{j}-P^{*} w_{j}\right] d \Gamma+\int_{\Omega}\left[w_{3}^{*}+\frac{\nu}{(1-\nu) \lambda^{2}} w_{\theta, \theta}^{*}\right] q d \Omega \\
& +\int_{\Omega}\left[\left(M_{\alpha \beta, \beta}^{*}-Q_{\alpha}^{*}\right) w_{\alpha}+Q_{\alpha, \alpha}^{*} w_{3}\right] d \Omega=0
\end{aligned}
$$

\subsubsection{Equação integral de contorno para placas espessas}

Analogamente ao que foi feito para a EIC de elasticidade, o processo anterior resulta no teorema da reciprocidade de Betti para placas de Reissner. Uma vez mais, para que seja possível aplicar o MEC, devemos escolher o estado ()* como aquele para um espaço fundamental de extensão infinita, carregado em um ponto $\mathbf{X}^{\prime}$ por cargas generalizadas concentradas e pontuais. Assim a equação (3.45) pode ser reescrita como: 


$$
\begin{aligned}
& \int_{\Gamma}\left[W_{i j}^{*}\left(\mathbf{X}^{\prime}, \mathbf{x}\right) p_{j}(\mathbf{x})-P_{i j}^{*}\left(\mathbf{X}^{\prime}, \mathbf{x}\right) w_{j}(\mathbf{x})\right] d \Gamma(\mathbf{x})+ \\
& \int_{\Omega}\left[W_{i 3}^{*}\left(\mathbf{X}^{\prime}, \mathbf{X}\right)+\frac{\nu}{(1-\nu) \lambda^{2}} W_{i \theta, \theta}^{*}\left(\mathbf{X}^{\prime}, \mathbf{X}\right)\right] q d \Omega(\mathbf{X})+ \\
& \int_{\Omega}\left(M_{i \alpha \beta, \beta}^{*}\left(\mathbf{X}^{\prime}, \mathbf{X}\right)-Q_{i \alpha}^{*}\left(\mathbf{X}^{\prime}, \mathbf{X}\right)\right) w_{\alpha}(\mathbf{X}) d \Omega(\mathbf{X})+ \\
& \int_{\Omega} Q_{\alpha, \alpha}^{*}\left(\mathbf{X}^{\prime}, \mathbf{X}\right) w_{3}(\mathbf{X}) d \Omega(\mathbf{X})=0
\end{aligned}
$$

na qual x é um ponto localizado exatamente no contorno e $\mathbf{X}$ é um ponto localizado no domínio. Como explicado anteriormente, para encontrar as soluções fundamentais do problema, deve-se fazer com que as equações governantes sejam resolvidas para uma carga pontual unitária, portanto:

$$
\begin{aligned}
M_{i \alpha \beta, \beta}^{*}\left(\mathbf{X}^{\prime}, \mathbf{X}\right)-Q_{i \alpha}^{*}\left(\mathbf{X}^{\prime}, \mathbf{X}\right) & =-\delta\left(\mathbf{X}^{\prime}, \mathbf{X}\right) \delta_{i \alpha} \\
Q_{\alpha, \alpha}^{*}\left(\mathbf{X}^{\prime}, \mathbf{X}\right) & =-\delta\left(\mathbf{X}^{\prime}, \mathbf{X}\right) \delta_{i 3}
\end{aligned}
$$

onde uma vez mais o delta de Dirac foi empregado. Usando a sua propriedade de seleção, é possível encontrar a equação integral de contorno para um ponto fonte no domínio, como sendo:

$$
\begin{aligned}
w_{j}\left(\mathbf{X}^{\prime}\right) & +\int_{\Gamma} P_{i j}^{*}\left(\mathbf{X}^{\prime}, \mathbf{x}\right) w_{j}(\mathbf{x}) d \Gamma(\mathbf{x})=\int_{\Gamma} W_{i j}^{*}\left(\mathbf{X}^{\prime}, \mathbf{x}\right) p_{j}(\mathbf{x}) d \Gamma(\mathbf{x}) \\
& +\int_{\Omega}\left[W_{i 3}^{*}\left(\mathbf{X}^{\prime}, \mathbf{X}\right)+\frac{\nu}{(1-\nu) \lambda^{2}} W_{i \theta, \theta}^{*}\left(\mathbf{X}^{\prime}, \mathbf{X}\right)\right] q(\mathbf{X}) d \Omega(\mathbf{X})
\end{aligned}
$$

onde $W_{i j}^{*}\left(\mathbf{X}^{\prime}, \mathbf{x}\right)$ e $P_{i j}^{*}\left(\mathbf{X}^{\prime}, \mathbf{x}\right)$ são as soluções fundamentais para deslocamentos e forças de superfície generalizadas, respectivamente. Ela representa um deslocamento no ponto $\mathbf{X}$ na direção $j$ devido a uma carga unitária aplicada em $\mathbf{X}^{\prime}$ na direção $i$.

\subsubsection{Soluções fundamentais}

A expressões para estas soluções fundamentais foram obtidas por [11] e são, para deslocamentos generalizados, dadas por:

$$
\begin{aligned}
W_{\alpha \beta}^{*} & =\frac{1}{8 \pi D(1-\nu)}\left\{[8 B(z)-(1-\nu)(2 \ln (z)-1)] \delta_{\alpha \beta}-[8 A(z)+2(1-\nu)] r_{, \alpha} r_{, \beta}\right\} \\
W_{\alpha 3}^{*} & =-W_{3 \alpha}^{*}=\frac{1}{8 \pi D}(2 \ln (z)-1) r r_{, \alpha} \\
W_{33}^{*} & =\frac{1}{8 \pi D(1-\nu) \lambda^{2}}\left[(1-\nu) z^{2}(\ln (z)-1)-8 \ln (z)\right]
\end{aligned}
$$


Para forças de superfície generalizadas, as expressões são:

$$
\begin{aligned}
P_{\gamma \beta}^{*}= & \frac{-1}{4 \pi r}\left[\left(4 A(z)+2 z K_{1}(z)+1-\nu\right)\left(\delta_{\alpha \gamma} r_{, n}+r_{, \alpha} n_{\gamma}\right)\right. \\
& \left.+(4 A(z)+1+\nu) r_{\gamma} n_{\alpha}-2\left(8 A(z)+2 z K_{1}(z)+1-\nu\right) r_{, \alpha} r_{, \gamma} r_{, n}\right] \\
P_{\gamma 3}^{*}= & \frac{\lambda^{2}}{2 \pi}\left[B(z) n_{\gamma}-A(z) r_{, \gamma} r_{, n}\right] \\
P_{3 \alpha}^{*}= & -\frac{(1-\nu)}{8 \pi}\left[\left(2 \frac{(1+\nu)}{(1-\nu)} \ln (z)-1\right) n_{\alpha}+2 r_{, \alpha} r_{, n}\right] \\
P_{33}^{*}= & -\frac{1}{2 \pi r} r_{, n}
\end{aligned}
$$

onde

$$
\begin{aligned}
& A(z)=K_{0}(z)+\frac{2}{z}\left[K_{1}(z)-\frac{1}{z}\right] \\
& B(z)=K_{0}(z)+\frac{1}{z}\left[K_{1}(z)-\frac{1}{z}\right]
\end{aligned}
$$

nas quais $K_{0}(z)$ e $K_{1}(z)$ são funções de Bessel modificadas, $z=\lambda r, r$ é a distância absoluta entre o ponto fonte e campo.

A equação (3.49) representa a identidade de Somigliana generalizada para placas de Reissner.

\subsubsection{Equação integral de contorno de placas especificada para pontos no contorno}

Levando o ponto $\mathbf{X}^{\prime}$ para um ponto localizado exatamente no contorno, na posição $\mathbf{x}^{\prime}$, e assumindo que os deslocamentos generalizados $w_{j}$ satisfazem o critério de continuidade de Hölder, a equação (3.49) pode então ser reescrita como:

$$
\begin{aligned}
C_{i j}\left(\mathbf{x}^{\prime}\right) w_{j}\left(\mathbf{x}^{\prime}\right) & +f_{\Gamma} P_{i j}^{*}\left(\mathbf{x}^{\prime}, \mathbf{x}\right) w_{j}(\mathbf{x}) d \Gamma(\mathbf{x})=\int_{\Gamma} W_{i j}^{*}\left(\mathbf{x}^{\prime}, \mathbf{x}\right) p_{j}(\mathbf{x}) d \Gamma(\mathbf{x}) \\
& +\int_{\Omega}\left[W_{i 3}^{*}\left(\mathbf{x}^{\prime}, \mathbf{X}\right)+\frac{\nu}{(1-\nu) \lambda^{2}} W_{i \theta, \theta}^{*}\left(\mathbf{x}^{\prime}, \mathbf{X}\right)\right] q(\mathbf{X}) d \Omega(\mathbf{X})
\end{aligned}
$$

na qual $f$ representa o valor da integral no sentido do valor principal de Cauchy e $C_{i j}\left(\mathbf{x}^{\prime}\right)$ são os termos livres oriundos do processo limite do tratamento da integral do termo $P_{i j}^{*}$. O valor de $C_{i j}\left(\mathbf{x}^{\prime}\right)$ é $\frac{1}{2} \delta_{i j}$ para contornos suaves. Esse termo pode também ser calculado através de considerações a respeito dos deslocamentos de corpo rígido generalizados. A equação (3.54) é um conjunto de 3 equações, duas para rotações em torno dos eixos $x_{1}$ e $x_{2}$, e uma para o deslocamento na direção $x_{3}$. 


\subsection{Discretização do problema e Método dos Elementos de Contorno}

As equações integrais de contorno (3.37) e (3.54), possuem complexidade alta e é possível encontrar soluções analíticas somente para problemas com geometria e condições de contorno simplificadas. Diante disso, e de maneira a ter uma metodologia de aplicação generalizada, deve-se abandonar a ambição de obter uma resposta exata e resolvê-lo através de uma aproximação. O método dos elementos de contorno é a técnica numérica aplicada para a resolução aproximada de equações integrais de contorno. O procedimento comumente adotado consiste em dividir o contorno do problema em vários pedaços ou elementos e realizar a integração separadamente para cada um deles [12] como mostrado na equação (3.55) e (3.56) para elasticidade plana e placas espessas, respectivamente.

$$
\begin{aligned}
C_{\alpha \beta}\left(\mathbf{x}^{\prime}\right) u_{\alpha}\left(\mathbf{x}^{\prime}\right) & =\sum_{e=1}^{N_{e}} \int_{\Gamma_{e}} U_{\alpha \beta}^{*}\left(\mathbf{x}^{\prime}, \mathbf{x}\right) t_{\beta}(\mathbf{x}) d \Gamma_{e}(\mathbf{x}) \\
& -\sum_{e=1}^{N_{e}} \int_{\Gamma_{e}} T_{\alpha \beta}^{*}\left(\mathbf{x}^{\prime}, \mathbf{x}\right) u_{\beta}(\mathbf{x}) d \Gamma_{e}(\mathbf{x}) \\
C_{i j}\left(\mathbf{x}^{\prime}\right) w_{j}\left(\mathbf{x}^{\prime}\right) & =\sum_{e=1}^{N_{e}} \int_{\Gamma_{e}} W_{i j}^{*}\left(\mathbf{x}^{\prime}, \mathbf{x}\right) p_{j}(\mathbf{x}) d \Gamma_{e}(\mathbf{x}) \\
& -\sum_{e=1}^{N_{e}} f_{\Gamma_{e}} P_{i j}^{*}\left(\mathbf{x}^{\prime}, \mathbf{x}\right) w_{j}(\mathbf{x}) d \Gamma_{e}(\mathbf{x})
\end{aligned}
$$

na qual foram desconsideradas as cargas de domínio e $N_{e}$ é o número total de elementos.

A Figura 3.2 mostra uma geometria genérica discretizada grosseiramente em elementos de contorno.
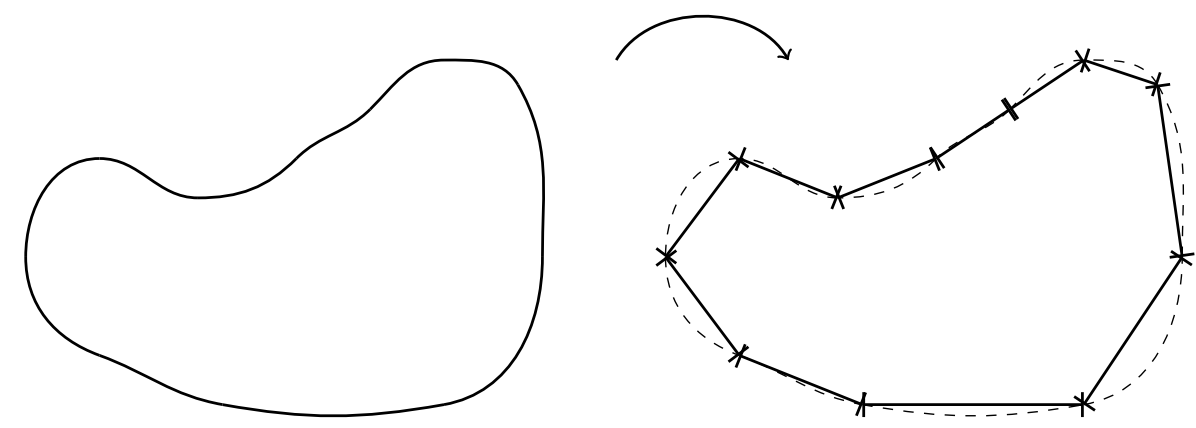

Figura 3.2: Discretização de um problema em elementos de contorno.

A variação das incógnitas do problema deve ser aproximada por valores tomados em um número de pontos chamados de nós e interpolados através de funções interpoladoras, 

a)
b)
c)
d)

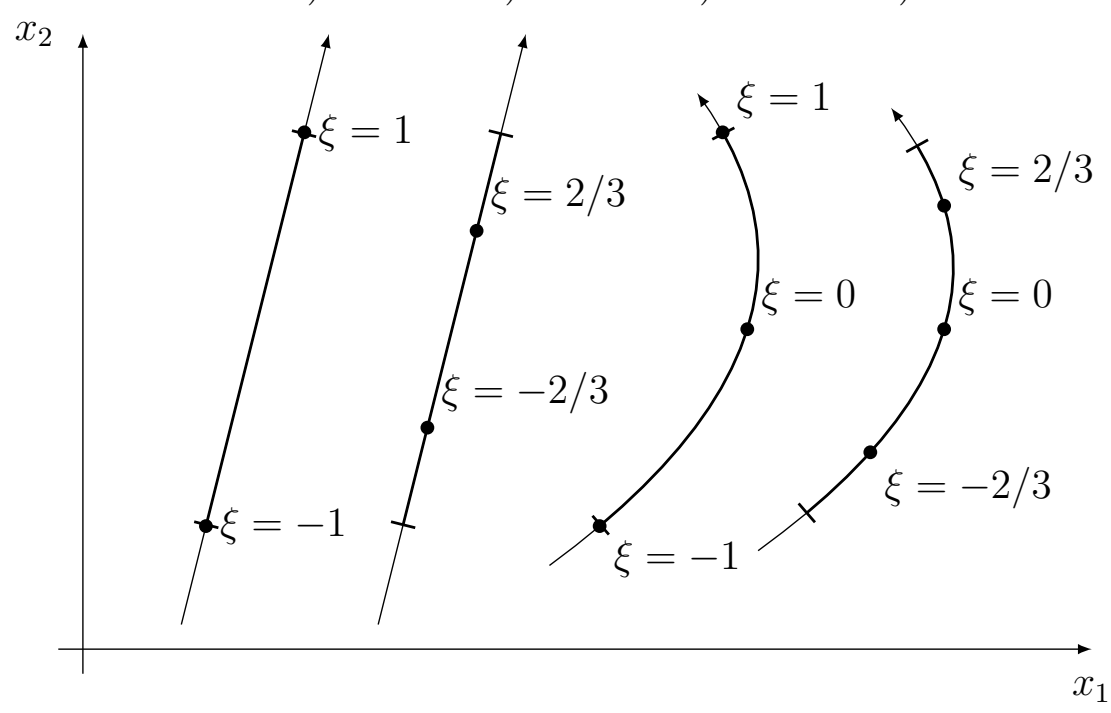

Figura 3.3: Representação de quatro tipos de elementos, a) lineares contínuos, b) lineares descontínuos, c) quadráticos contínuos, d) quadráticos descontínuos.

conhecidas como funções de forma. Uma vez que os valores nodais são parâmetros, as variáveis do problema podem ser tiradas da integral. Os termos restantes são então integrados, o que leva a uma equação puramente algébrica. Para que seja possível resolver o problema, deve-se escrever um número de equações correspondente ao de valores nodais, isso é feito alterando a posição do fonte. A técnica mais comum é utilizar os próprios nós para esse posicionamento mas outros métodos podem também ser usados. O passo seguinte é utilizar as condições de contorno do problema e resolver o sistema de equações resultante usando, por exemplo, eliminação gaussiana. Com posse dos resultados nodais, é possível calcular as variáveis do problema em qualquer ponto do domínio.

\subsubsection{Funções de forma}

Para descrever a geometria do problema e também para aproximar o campo de deslocamentos e forças de superfície, funções de forma devem ser utilizadas. Estas expressões são obtidas tipicamente através da seguinte lógica: a função $\Phi_{i}$ é deduzida de tal maneira que tenha valor 1 no nó $i$ e 0 nos demais. As funções de forma variam também de acordo com a posição de seus nós no espaço parametrizado $\xi=[-1,1]$ e quanto à ordem da função interpoladora. Considere a Figura 3.3, nela são mostrados exemplos de elementos de contorno lineares e quadráticos, contínuos e descontínuos:

Tomando o exemplo da função de forma linear descontínua com nós localizados em $\xi=[-2 / 3,2 / 3]$, partindo de uma equação linear genérica: 


$$
N(\xi)=a+b \xi
$$

para a primeira função de forma tem-se:

$$
\begin{gathered}
N_{1}(\xi=-2 / 3)=a+b(-2 / 3)=1 \\
N_{1}(\xi=2 / 3)=a+b(2 / 3)=0
\end{gathered}
$$

o que resulta em $a=1 / 2$ e $b=-3 / 4$, para a segunda função de forma:

$$
\begin{gathered}
N_{2}(\xi=-2 / 3)=a+b(-2 / 3)=0 \\
N_{2}(\xi=2 / 3)=a+b(2 / 3)=1
\end{gathered}
$$

o que resulta em $a=1 / 2$ e $b=3 / 4$. Assim as funções de forma lineares descontínuas com nós localizados em $\xi=[-2 / 3,2 / 3]$ do espaço paramétrico são:

$$
\begin{aligned}
& N_{1}^{d}(\xi)=\frac{3}{4}\left(\frac{2}{3}-\xi\right) \\
& N_{2}^{d}(\xi)=\frac{3}{4}\left(\frac{2}{3}+\xi\right)
\end{aligned}
$$

na qual o sobre escrito $d$ é usado para diferenciá-las das funções de forma lineares contínuas.

Quando é usada a mesma função interpoladora para aproximar a geometria e as variáveis nodais tem-se aquilo que é conhecido como elemento isoparamétrico. Neste trabalho contudo, serão utilizados elementos diferentes, contínuos para a geometria e descontínuos para as variáveis nodais. Isto é feito por conta da maior facilidade da implementação e da conveniência de funções interpoladoras descontínuas para o tratamento das hiper singularidades. Para elementos lineares então, além de (3.60), as funções de forma lineares contínuas são dadas por:

$$
\begin{aligned}
& N_{1}^{c}(\xi)=\frac{1}{2}(1-\xi) \\
& N_{2}^{c}(\xi)=\frac{1}{2}(1+\xi)
\end{aligned}
$$

Para elementos quadráticos descontínuos, que podem ser obtidos por procedimento análogo, as funções de forma são dadas por: 


$$
\begin{aligned}
N_{1}^{d}(\xi) & =\xi\left(\frac{9}{8} \xi-\frac{3}{4}\right) \\
N_{2}^{d}(\xi) & =1-\frac{9}{4} \xi^{2} \\
N_{3}^{d}(\xi) & =\xi\left(\frac{9}{8} \xi+\frac{3}{4}\right)
\end{aligned}
$$

e para elementos quadráticos contínuos:

$$
\begin{aligned}
N_{1}^{c}(\xi) & =\frac{1}{2} \xi(\xi-1) \\
N_{2}^{c}(\xi) & =1-\xi^{2} \\
N_{3}^{c}(\xi) & =\frac{1}{2} \xi(\xi+1)
\end{aligned}
$$

Uma vez de posse das funções interpoladoras, pode-se agora expressar a geometria e as variáveis nodais em função de $\xi$ e dos valores nodais (constantes) destas grandezas. Para o caso da geometria, temos:

$$
\begin{aligned}
& x_{i}(\xi)=N_{1}^{c}(\xi) x_{i}^{1}+N_{2}^{c}(\xi) x_{i}^{2} \\
& x_{i}(\xi)=N_{1}^{c}(\xi) x_{i}^{1}+N_{2}^{c}(\xi) x_{i}^{2}+N_{3}^{c}(\xi) x_{i}^{3}
\end{aligned}
$$

Para o caso das variáveis nodais a interpolação pode ser representada de uma forma diferente, mais conveniente para a posterior integração. Cada componente dos vetores nodais deve ser interpolada pelas funções de forma, em uma representação matricial para o caso da elasticidade plana, a variação dos deslocamentos em um elemento é dada por:

$$
\begin{aligned}
& \left\{\begin{array}{l}
u_{1}(\xi) \\
u_{2}(\xi)
\end{array}\right\}=\Phi_{d}^{1}(\xi)\left\{\begin{array}{l}
u_{1}^{1} \\
u_{2}^{1} \\
u_{1}^{2} \\
u_{2}^{2}
\end{array}\right\} \\
& \left\{\begin{array}{l}
u_{1}^{1} \\
u_{1}(\xi) \\
u_{2}(\xi)
\end{array}\right\}=\Phi_{d}^{2}(\xi)\left\{\begin{array}{l}
u_{2}^{1} \\
u_{1}^{2} \\
u_{2}^{2} \\
u_{1}^{3} \\
u_{2}^{3}
\end{array}\right\}
\end{aligned}
$$

e para as forças de superfície, 


$$
\begin{aligned}
& \left\{\begin{array}{l}
t_{1}(\xi) \\
t_{2}(\xi)
\end{array}\right\}=\Phi_{d}^{1}(\xi)\left\{\begin{array}{l}
t_{1}^{1} \\
t_{2}^{1} \\
t_{1}^{2} \\
t_{2}^{2}
\end{array}\right\} \\
& \left\{\begin{array}{c}
t_{1}^{1} \\
t_{2}^{1} \\
t_{2}(\xi)
\end{array}\right\}=\Phi_{d}^{2}(\xi)\left\{\begin{array}{l}
t_{1}^{2} \\
t_{2}^{2} \\
t_{1}^{3} \\
t_{2}^{3}
\end{array}\right\}
\end{aligned}
$$

nas quais:

$$
\begin{aligned}
\Phi_{d}^{1}(\xi) & =\left[\begin{array}{cccc}
N_{1}^{d}(\xi) & 0 & N_{2}^{d}(\xi) & 0 \\
0 & N_{1}^{d}(\xi) & 0 & N_{2}^{d}(\xi)
\end{array}\right] \\
\Phi_{d}^{2}(\xi) & =\left[\begin{array}{cccccc}
N_{1}^{d}(\xi) & 0 & N_{2}^{d}(\xi) & 0 & N_{3}^{d}(\xi) & \\
0 & N_{1}^{d}(\xi) & 0 & N_{2}^{d}(\xi) & 0 & N_{3}^{d}(\xi)
\end{array}\right]
\end{aligned}
$$

São as matrizes de funções de forma para vetores com 2 componentes. Neste caso o sobre escrito é o grau da função interpoladora e o sub escrito o tipo de função de forma, se contínua $c$ ou descontínua $d$.

Para o caso da formulação de placas espessas a aproximação fica da seguinte forma:

$$
\left\{\begin{array}{l}
w_{1}(\xi) \\
w_{2}(\xi) \\
w_{3}(\xi)
\end{array}\right\}=\Xi_{d}^{1}(\xi)\left\{\begin{array}{c}
w_{1}^{1} \\
w_{2}^{1} \\
w_{3}^{1} \\
w_{1}^{2} \\
w_{2}^{2} \\
w_{3}^{2}
\end{array}\right\}
$$

para os deslocamentos generalizados e 


$$
\left\{\begin{array}{c}
w_{1}(\xi) \\
w_{2}(\xi) \\
w_{3}(\xi)
\end{array}\right\}=\Xi_{d}^{2}(\xi)\left\{\begin{array}{c}
w_{1}^{1} \\
w_{2}^{1} \\
w_{3}^{1} \\
w_{1}^{2} \\
w_{2}^{2} \\
w_{3}^{2} \\
w_{1}^{3} \\
w_{2}^{3} \\
w_{3}^{3}
\end{array}\right\}
$$

para as forças de superfície generalizadas,

$$
\begin{aligned}
& \left\{\begin{array}{l}
p_{1}(\xi) \\
p_{2}(\xi) \\
p_{3}(\xi)
\end{array}\right\}=\Xi_{d}^{1}(\xi)\left\{\begin{array}{l}
p_{1}^{1} \\
p_{2}^{1} \\
p_{3}^{1} \\
p_{1}^{2} \\
p_{2}^{2} \\
p_{3}^{2}
\end{array}\right\} \\
& \left\{\begin{array}{c}
p_{1}^{1} \\
p_{1}^{1} \\
p_{2}(\xi) \\
p_{3}(\xi)
\end{array}\right\}=\Xi_{d}^{2}(\xi)\left\{\begin{array}{l}
p_{3}^{1} \\
p_{1}^{2} \\
p_{2}^{2} \\
p_{3}^{2} \\
p_{1}^{3} \\
p_{2}^{3} \\
p_{3}^{3}
\end{array}\right\}
\end{aligned}
$$

nas quais:

$$
\begin{aligned}
& \Xi_{d}^{1}(\xi)=\left[\begin{array}{cccccc}
N_{1}^{d}(\xi) & 0 & 0 & N_{2}^{d}(\xi) & 0 & 0 \\
0 & N_{1}^{d}(\xi) & 0 & 0 & N_{2}^{d}(\xi) & 0 \\
0 & 0 & N_{1}^{d}(\xi) & 0 & 0 & N_{2}^{d}(\xi)
\end{array}\right] \\
& \Xi_{d}^{2}(\xi)= \\
& {\left[\begin{array}{ccccccccc}
N_{1}^{d}(\xi) & 0 & 0 & N_{2}^{d}(\xi) & 0 & 0 & N_{3}^{d}(\xi) & 0 & 0 \\
0 & N_{1}^{d}(\xi) & 0 & 0 & N_{2}^{d}(\xi) & 0 & 0 & N_{3}^{d}(\xi) & 0 \\
0 & 0 & N_{1}^{d}(\xi) & 0 & 0 & N_{2}^{d}(\xi) & 0 & 0 & N_{3}^{d}(\xi)
\end{array}\right]}
\end{aligned}
$$


São as matrizes de funções de forma para vetores com 3 componentes.

\subsubsection{Integração dos elementos}

De posse das equações descritas na seção anterior, deve-se alterar o domínio de integração, para isso é necessário calcular o jacobiano, que é dado por:

$$
J(\xi)=\sqrt{\sum_{i=1}^{\operatorname{dim}}\left(\frac{d N_{j}}{d \xi} x_{i}^{j}\right)^{2}}
$$

na qual a convenção de somatório da notação indicial para o índice $j$ é utilizada e dim é a dimensão do problema.

Para as integrais contidas no lado direito da equação (3.55) para um caso bidimensional de elasticidade plana, a inclusão das funções de forma lineares e alteração do domínio de integração resulta em:

$$
\int_{\Gamma_{e}} U_{\alpha \beta}^{*}\left(\mathbf{x}^{\prime}, \mathbf{x}\right) t_{\beta}(\mathbf{x}) d \Gamma_{e}=\int_{-1}^{1} U_{\alpha \beta}^{*}\left(\mathbf{x}^{\prime}(\xi), \mathbf{x}(\xi)\right) \Phi_{d}^{1}(\xi) J(\xi) d \xi\left\{\begin{array}{c}
t_{1}^{1} \\
t_{2}^{1} \\
t_{1}^{2} \\
t_{2}^{2}
\end{array}\right\}
$$

e

$$
\int_{\Gamma_{e}} T_{\alpha \beta}^{*}\left(\mathbf{x}^{\prime}, \mathbf{x}\right) u_{\beta}(\mathbf{x}) d \Gamma_{e}=\int_{-1}^{1} T_{\alpha \beta}^{*}\left(\mathbf{x}^{\prime}(\xi), \mathbf{x}(\xi)\right) \Phi_{d}^{1}(\xi) J(\xi) d \xi\left\{\begin{array}{c}
u_{1}^{1} \\
u_{2}^{1} \\
u_{1}^{2} \\
u_{2}^{2}
\end{array}\right\}
$$

Para o caso da formulação com elementos quadráticos, as relações se tornam:

$$
\int_{\Gamma_{e}} U_{\alpha \beta}^{*}\left(\mathbf{x}^{\prime}, \mathbf{x}\right) t_{\beta}(\mathbf{x}) d \Gamma_{e}=\int_{-1}^{1} U_{\alpha \beta}^{*}\left(\mathbf{x}^{\prime}(\xi), \mathbf{x}(\xi)\right) \Phi_{d}^{2}(\xi) J(\xi) d \xi\left\{\begin{array}{c}
t_{1}^{1} \\
t_{2}^{1} \\
t_{1}^{2} \\
t_{2}^{2} \\
t_{1}^{3} \\
t_{2}^{3}
\end{array}\right\}
$$

e 


$$
\int_{\Gamma_{e}} T_{\alpha \beta}^{*}\left(\mathbf{x}^{\prime}, \mathbf{x}\right) u_{\beta}(\mathbf{x}) d \Gamma_{e}=\int_{-1}^{1} T_{\alpha \beta}^{*}\left(\mathbf{x}^{\prime}(\xi), \mathbf{x}(\xi)\right) \Phi_{d}^{2}(\xi) J(\xi) d \xi\left\{\begin{array}{c}
u_{1}^{1} \\
u_{2}^{1} \\
u_{1}^{2} \\
u_{2}^{2} \\
u_{3}^{1} \\
u_{3}^{2}
\end{array}\right\}
$$

Repetindo o mesmo procedimento, agora para as integrais contidas na equação (3.56) da formulação de placas espessas, a inclusão das funções de forma lineares e alteração do domínio de integração resulta em:

$$
\int_{\Gamma_{e}} W_{i j}^{*}\left(\mathbf{x}^{\prime}, \mathbf{x}\right) p_{j}(\mathbf{x}) d \Gamma_{e}=\int_{-1}^{1} W_{i j}^{*}\left(\mathbf{x}^{\prime}(\xi), \mathbf{x}(\xi)\right) \Xi_{d}^{1}(\xi) J(\xi) d \xi\left\{\begin{array}{c}
p_{1}^{1} \\
p_{2}^{1} \\
p_{3}^{1} \\
p_{1}^{2} \\
p_{2}^{2} \\
p_{3}^{2}
\end{array}\right\}
$$

e

$$
\int_{\Gamma_{e}} P_{i j}^{*}\left(\mathbf{x}^{\prime}, \mathbf{x}\right) w_{j}(\mathbf{x}) d \Gamma_{e}=\int_{-1}^{1} P_{i j}^{*}\left(\mathbf{x}^{\prime}(\xi), \mathbf{x}(\xi)\right) \Xi_{d}^{1}(\xi) J(\xi) d \xi\left\{\begin{array}{c}
w_{1}^{1} \\
w_{2}^{1} \\
w_{3}^{1} \\
w_{1}^{2} \\
w_{2}^{2} \\
w_{3}^{2}
\end{array}\right\}
$$

Para o caso da formulação com elementos quadráticos, as relações se tornam:

$$
\int_{\Gamma_{e}} W_{i j}^{*}\left(\mathbf{x}^{\prime}, \mathbf{x}\right) p_{j}(\mathbf{x}) d \Gamma_{e}=\int_{-1}^{1} W_{i j}^{*}\left(\mathbf{x}^{\prime}(\xi), \mathbf{x}(\xi)\right) \Xi_{d}^{2}(\xi) J(\xi) d \xi\left\{\begin{array}{c}
p_{1}^{1} \\
p_{2}^{1} \\
p_{3}^{1} \\
p_{1}^{2} \\
p_{2}^{2} \\
p_{3}^{2} \\
p_{1}^{3} \\
p_{2}^{3} \\
p_{3}^{3}
\end{array}\right\}
$$




$$
\int_{\Gamma_{e}} P_{i j}^{*}\left(\mathbf{x}^{\prime}, \mathbf{x}\right) w_{j}(\mathbf{x}) d \Gamma_{e}=\int_{-1}^{1} P_{i j}^{*}\left(\mathbf{x}^{\prime}(\xi), \mathbf{x}(\xi)\right) \Xi_{d}^{2}(\xi) J(\xi) d \xi\left\{\begin{array}{c}
w_{1}^{1} \\
w_{2}^{1} \\
w_{3}^{1} \\
w_{1}^{2} \\
w_{2}^{2} \\
w_{3}^{2} \\
w_{1}^{3} \\
w_{2}^{3} \\
w_{3}^{3}
\end{array}\right\}
$$

Essas integrais podem ser solucionadas utilizando métodos analíticos em alguns casos, porém a dificuldade de implementação faz com que métodos de integração numérica como a quadratura gaussiana sejam amplamente aplicados. Uma cuidadosa avaliação destas integrais deve também ser feita sempre que o elemento a ser integrado contenha o nó onde está posicionado o ponto fonte.

\subsubsection{Integração Numérica}

As integrais das formulações de elementos de contorno apresentadas neste trabalho possuem, em sua maioria, a seguinte forma (3.86):

$$
I=\int_{-1}^{1} F_{i j}\left(\xi^{\prime}, \xi\right) \Phi_{i}(\xi) J(\xi) d \xi
$$

na qual $F_{i j}$ é uma solução fundamental genérica, $\Phi_{i}$ é uma função de forma, $J(\xi)$ é o jacobiano da transformação para o espaço paramétrico e $\xi$ é o parâmetro desse espaço. De maneira geral essas integrais possuem uma solução analítica complicada, as vezes possível [39, 40], de tal maneira que para generalização da técnica convencionou-se utilizar métodos de integração numérica. O primeiro passo para isso, já foi mostrado na Equação (3.86), que é a mudança de variáveis para o espaço isoparamétrico. Após isso, técnicas de integração numéricas podem ser facilmente aplicadas.

Entre as técnicas de integração mais conhecidas, podemos citar a regra do trapézio, regra de Simpson e a quadratura de Gauss. Devido a sua alta precisão e seu relativo baixo custo computacional a maioria dos métodos utiliza a quadratura gaussiana. A aproximação numérica de uma integral, de maneira geral, é feita através de um somatório da função avaliada em certos pontos, multiplicadas por funções peso. Como exemplo, avalia-se a função genérica: 


$$
\int_{-1}^{1} f(\xi) d \xi
$$

aproximada por:

$$
I=\sum_{i=1}^{n} f\left(\xi_{i}\right) W_{i}
$$

onde $W_{i}$ são as funções peso, $\xi_{i}$ são as coordenadas paramétricas correspondentes aos pontos de integração, cujo total é $n$. Utilizando a regra do trapézio com $n=1$, a resolução seria:

$$
\int_{-1}^{1} f(\xi) d \xi \approx f(-1)+f(1)
$$

em que os pontos de integração são -1 e 1 e os pesos são iguais, de valor unitário. Como dito anteriormente, a quadratura gaussiana possui uma maior precisão, para um número semelhante de pontos de integração, quando comparada às regras de NewtonCotes. Neste método é assumido que a função a integrar pode ser escrita como um polinômio na forma:

$$
f(\xi)=a_{0}+a_{1} \xi+a_{2} \xi^{2}+\ldots+a_{p} \xi^{p}
$$

na qual os coeficientes podem ser ajustados de maneira a obter o melhor ajuste de $f(\xi)$.

Na quadratura Gaussiana a localização dos pontos de integração é escolhida de maneira que a equação (3.88) resulte no valor exato, se a função $f(x)$ for um polinômio de grau $2 n-1$ ou menos, de tal maneira que [41]:

$$
\int_{a}^{b} P_{m}(x) d x=\sum_{i=1}^{n} P_{m}\left(\xi_{i}\right) W_{i}, m \leq 2 n-1
$$

O cálculo dos pontos e pesos de Gauss pode ser feito substituindo $P_{1}(x)=1$, $P_{2}(x)=x \ldots, P_{2 n-1}(x)=x^{2 n-1}$ na equação (3.91) e resolvendo as $2 n$ equações resultantes para $W_{i}$ e $x_{i}, i=1,2, \ldots, n$.

A Tabela 3.1 mostra o grau do polinômio que pode ser integrado analiticamente com a quantidade de pontos de integração correspondente.

Desta maneira a integração da equação (3.86) será dada por:

$$
I=\int_{-1}^{1} F_{i j}\left(\xi^{\prime}, \xi\right) \Phi_{i}(\xi) J(\xi) d \xi \approx \sum_{k=1}^{n} F_{i j}\left(\xi^{\prime}, \xi_{k}\right) \Phi_{i}\left(\xi_{k}\right) J\left(\xi_{k}\right) W_{k}
$$

onde $\xi_{k}$ são os pontos e $W_{k}$ os pesos da quadratura Gaussiana. A equação (3.92) poderá ser aplicada para todas as integrais apresentadas neste capítulo, exceto quando o ponto 
Tabela 3.1: Quantidade de pontos de Gauss de acordo com o grau do polinômio que pode ser integrado exatamente.

\begin{tabular}{cc}
\hline $\mathrm{N}^{\mathrm{O}}$ de pontos de Gauss & Grau do polinômio \\
\hline 1 & 1 \\
2 & 3 \\
3 & 5 \\
\hline
\end{tabular}

fonte estiver contido no elemento integrado, o que nos leva aos procedimentos a serem descritos na seção seguinte.

\subsection{Tratamento de singularidades}

Quando o ponto fonte pertence ao elemento que está sendo integrado, surgem dois tipos de singularidades nas expressões (3.78), (3.79), (3.82) e (3.83). Aquelas envolvendo os termos $T_{\alpha \beta}^{*}$ e $P_{i j}^{*}$ são fortemente singulares de ordem $\mathrm{O}\left(r^{-1}\right)$ e aquelas envolvendo os termos $U_{\alpha \beta}^{*}$ e $W_{i j}^{*}$ são fracamente singulares de ordem $\mathrm{O}(\ln (r))$. A abordagem utilizada neste trabalho é a transformação de coordenadas desenvolvida por Telles [42]. Esse procedimento é suficiente para lidar com as integrais singulares de ordem $\mathrm{O}(\ln (r))$. Para o cálculo das integrais contendo os termos $T_{\alpha \beta}^{*}, P_{i j}^{*}$ foram feitas considerações de corpo rígido, uma maneira indireta de realização dos cálculos. Finalmente, para as derivadas de soluções fundamentais, ou combinações das mesmas, os procedimentos descritos acima não são efetivos e técnicas como a subtração de singularidades ou tratamento analítico, devem ser adotados. A seguir, serão mostradas as descrições de algumas das técnicas utilizadas para o tratamento de singularidades.

\subsubsection{Técnica de subdivisão de elementos}

Esta técnica consiste na subdivisão do elemento a ser integrado em sub intervalos. Ela foi desenvolvida por [21] pra problemas bidimensionais e tridimensionais, relacionando o número de divisões ao maior erro na quadratura gaussiana. Dividindo o domínio de integração em $M$ intervalos resulta em [43]:

$$
\int_{-1}^{1} f(\xi) d \xi=\frac{1}{M} \sum_{m=1}^{M} \sum_{i=1}^{n} f\left(\bar{\xi}_{i}\right) W_{i}
$$

na qual:

$$
\bar{\xi}_{i}=\frac{1}{M}\left(M-2 n+1+\xi_{i}\right)
$$




\subsubsection{Técnica de transformação de variáveis}

Esta técnica também conhecida como transformada de Telles, foi obtida em [42], para tratar de integrais quase e fracamente singulares. Ela envolve uma mudança de variáveis, feita de tal maneira, que o jacobiano é convenientemente igualado a zero, no caso de singularidades fracas ou alterado para um valor ótimo no caso de quase singularidades. O efeito desta transformação é que os pontos de Gauss se concentram nas proximidades da singularidade o que melhora a precisão nas regiões de rápida variação da função [43].

A transformação de Telles deve ser deduzida de tal maneira, que o jacobiano resultante cancele a singularidade ou torne seu efeito menos intenso, o suficiente para que a quadratura gaussiana padrão a calcule com precisão. Telles [42] propôs uma transformação baseada em um polinômio de grau 3 para lidar com as singularidades no intervalo de integração $[-1,1]$. Considerando o seguinte polinômio de grau 3 genérico:

$$
\eta(\xi)=a \xi^{3}+b \xi^{2}+c \xi+d
$$

Impondo as seguinte condições:

$$
\begin{aligned}
\left.\frac{d^{2} \eta}{d \xi^{2}}\right|_{\eta^{\prime}} & =0 \\
\left.\frac{d \eta}{d \xi}\right|_{\eta^{\prime}} & =0 \\
\eta(1) & =1 \\
\eta(-1) & =-1
\end{aligned}
$$

As constantes contidas na equação (3.95) podem ser encontradas e são iguais a:

$$
\begin{aligned}
a & =\frac{1}{1+\bar{\xi}^{2}} \\
b & =\frac{-3 \bar{\xi}}{1+\bar{\xi}^{2}} \\
c & =\frac{3 \bar{\xi}^{2}}{1+\bar{\xi}^{2}} \\
d & =-b
\end{aligned}
$$

onde $\bar{\xi}$ é o valor de $\xi$ que satisfaz:

$$
\eta(\bar{\xi})=\bar{\eta}
$$

dado por: 


$$
\bar{\xi}=\sqrt[3]{\bar{\eta} \eta^{*}+\left|\eta^{*}\right|}+\sqrt[3]{\bar{\eta} \eta^{*}-\left|\eta^{*}\right|}+\bar{\eta}
$$

na qual $\bar{\xi}$ é a posição do ponto fonte no domínio paramétrico e,

$$
\eta^{*}=\bar{\eta}^{2}-1
$$

O jacobiano neste caso é dado por:

$$
J=\frac{d \eta}{d \xi}=\frac{3(\xi-\bar{\xi})^{2}}{1+\bar{\xi}^{2}}
$$

\subsubsection{Considerações de corpo rígido generalizados}

As integrais contidas nos termos $T_{\alpha \beta}^{*}$ e $P_{i j}^{*}$ apresentam singularidades fortes quando o ponto fonte pertence ao elemento sendo integrado. As técnicas descritas acima não são suficientes para garantir a precisão necessária à integração. Felizmente, este cálculo pode ser feito indiretamente através das conhecidas considerações de corpo rígido.

Assim, os termos a serem subtraídos da diagonal da matriz $\mathbf{H}$ são dados para a elasticidade plana por [12]:

$$
C_{\alpha \beta}^{e}\left(\mathbf{x}^{\prime}\right)=-\int_{\Gamma} T_{\alpha \beta}\left(\mathbf{x}^{\prime}, \mathbf{x}\right) d \Gamma\left(\mathbf{x}^{\prime}\right) \quad \alpha, \beta=1,2 .
$$

e para a formulação de placas por:

$$
\begin{aligned}
C_{i \alpha}^{p}\left(\mathbf{x}^{\prime}\right) & =-\int_{\Gamma}\left[P_{i \alpha}\left(\mathbf{x}^{\prime}, \mathbf{x}\right)+\left(-r_{\alpha}\right) P_{i 3}\left(\mathbf{x}^{\prime}, \mathbf{x}\right)\right] d \Gamma\left(\mathbf{x}^{\prime}\right) \\
C_{i 3}^{p}\left(\mathbf{x}^{\prime}\right) & =-\int_{\Gamma} P_{i 3}\left(\mathbf{x}^{\prime}, \mathbf{x}\right) d \Gamma\left(\mathbf{x}^{\prime}\right) \quad i=1,2,3 .
\end{aligned}
$$

onde os termos $C_{\alpha \beta}^{e}$ e $C_{i j}^{p}$ incluem as respectivas sub matrizes diagonais e termos livres $C_{i j}$ [13]. Os sobrescritos e e $p$ indicam as constantes relativas respectivamente a elasticidade plana e placas deformáveis por cisalhamento. 


\section{FORMULAÇÕES DO MEC PARA DRILLING ROTATION}

\subsection{Introdução}

Neste capítulo, duas abordagens diferentes serão apresentadas para o cálculo da rotação em torno do eixo $x_{3}$ com o MEC. A primeira desenvolvida pelo coorientador desta tese [25], tem como base um enriquecimento da função de forma pela rotação e o uso de conceitos da teoria de partição da unidade (partition of unity). A segunda abordagem, desenvolvida ao longo desta tese de doutorado, se baseia na definição da rotação do ponto de vista da teoria da elasticidade, e seu desenvolvimento parte da equação integral de contorno em um procedimento semelhante àquele aplicado para se obter, por exemplo, a equação integral para tensões ou deformações.

\subsection{PUBEM e XBEM}

Uma das primeiras abordagens bem sucedidas ao problema da inclusão da rotação $\omega_{3}$ em uma formulação do MEC foi obtida por Leung \& Baiz (2013) [25]. A técnica utilizada se baseou em um procedimento análogo àquele aplicado no MEF, que consiste em alterar de maneira conveniente a função de forma com o intuito de incluir essa rotação. A primeira parte do título desta seção, PUBEM (Partition of Unity Boundary Element Method), está relacionado à partição da unidade empregada no desenvolvimento desta formulação. A segunda parte, XBEM (Extended Boundary Element Method), é devido ao enriquecimento da formulação com a rotação, em uma alusão ao XFEM.

Em [25], elementos de contorno de quatro tipos foram utilizados:

- Elementos lineares contínuos:

$$
\begin{aligned}
& N_{1}^{c}=\frac{1}{2}(1-\xi) \\
& N_{2}^{c}=\frac{1}{2}(1+\xi)
\end{aligned}
$$

- Elementos lineares descontínuos:

$$
\begin{aligned}
& N_{1}^{d}=\frac{3}{4}\left(\frac{2}{3}-\xi\right) \\
& N_{2}^{d}=\frac{3}{4}\left(\frac{2}{3}+\xi\right)
\end{aligned}
$$


- Elementos lineares semi descontínuos, contínuos à direita:

$$
\begin{aligned}
N_{1}^{s d d} & =\frac{3}{5}(1-\xi) \\
N_{2}^{s d d} & =\frac{3}{5}\left(\frac{2}{3}+\xi\right)
\end{aligned}
$$

- Elementos lineares semi descontínuos, contínuos à esquerda:

$$
\begin{aligned}
N_{1}^{\text {sde }} & =\frac{3}{5}\left(\frac{2}{3}-\xi\right) \\
N_{2}^{\text {sde }} & =\frac{3}{5}(1+\xi)
\end{aligned}
$$

A partir destas expressões, funções de forma enriquecidas foram desenvolvidas usando as estratégias apresentadas em [27], no qual foi mostrado que as componentes normal $u_{n}$ e tangencial $u_{t}$ dos deslocamentos podem ser representadas da seguinte maneira:

$$
\begin{aligned}
& u_{n}=a_{1}+a_{2} \xi+a_{3} \xi^{2} \\
& u_{t}=a_{4}+a_{5} \xi
\end{aligned}
$$

onde $\xi$ é a coordenada paramétrica, mostrada na Figura 4.1. Os cinco coeficientes devem ser avaliados a partir dos conectores nos extremos do elemento. Contudo, existem seis conectores, dois deslocamentos e uma rotação para cada um dos nós e o problema fica sobre determinado. Adotando a estratégia usada por [27], são consideradas as seguintes condições de contorno:

$$
\begin{aligned}
u_{n \mid \xi=-1} & =u_{n}^{1} \\
u_{t \mid \xi=-1} & =u_{t}^{1} \\
u_{n \mid \xi=1} & =u_{n}^{2} \\
u_{t \mid \xi=1} & =u_{t}^{2}
\end{aligned}
$$

onde o sobrescrito, neste caso, indica o número do nó. A quinta condição de contorno é escolhida como sendo a diferença entre as derivadas em relação a normal dos deslocamentos nos extremos do elemento:

$$
{\frac{\partial u_{n}}{\partial \xi}}_{\mid \xi=1}-{\frac{\partial u_{n}}{\partial \xi}}_{\mid \xi=-1}=\psi^{1}-\psi^{2}
$$

onde $\psi^{i}$ é uma grandeza relacionada à rotação. Esta aproximação leva ao desenvolvimento do elemento que ficou conhecido como triângulo de Allman. Usando as condições 


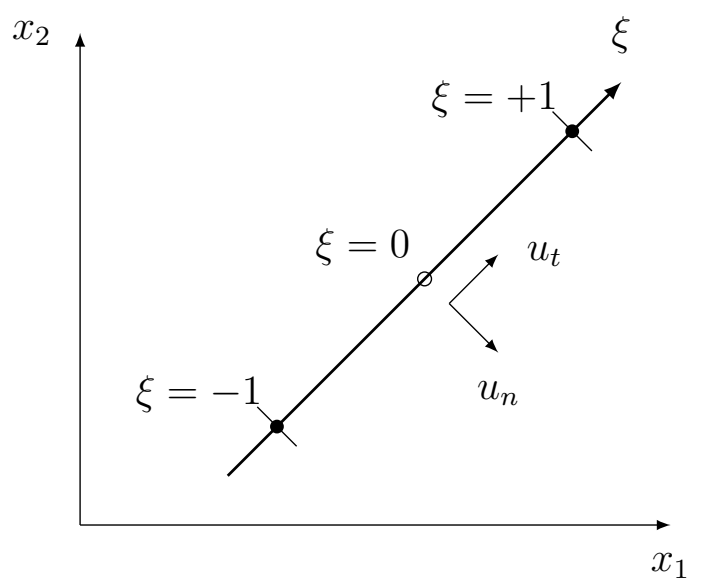

Figura 4.1: Representação esquemática da coordenada paramétrica $\xi$ no plano $x_{1}-x_{2}$ e do sistema local $n-t$ para o caso de um elemento linear contínuo.

de contorno (4.11) e (4.12) é possível escrever o campo de deslocamentos em função dos conectores da seguinte maneira:

$$
\begin{aligned}
\left\{\begin{array}{l}
u_{n} \\
u_{t}
\end{array}\right\}= & {\left[\begin{array}{ccc}
\frac{1}{2}-\frac{\xi}{2} & 0 & -\frac{1}{4}+\frac{\xi^{2}}{4} \\
0 & \frac{1}{2}-\frac{\xi}{2} & 0
\end{array}\right]\left\{\begin{array}{l}
u_{n}^{1} \\
u_{t}^{1} \\
\psi^{1}
\end{array}\right\}+} \\
& {\left[\begin{array}{ccc}
\frac{1}{2}+\frac{\xi}{2} & 0 & \frac{1}{4}-\frac{\xi^{2}}{4} \\
0 & \frac{1}{2}+\frac{\xi}{2} & 0
\end{array}\right]\left\{\begin{array}{l}
u_{n}^{2} \\
u_{t}^{2} \\
\psi^{2}
\end{array}\right\} }
\end{aligned}
$$

A equação (4.13) está escrita em coordenadas locais como mostrado na Figura 4.1 e deve ser transformada para o sistema global, dessa maneira as funções de forma definitivas podem ser encontradas. A transformação pode ser feita pela seguinte equação:

$$
\left\{\begin{array}{l}
u_{1} \\
u_{2} \\
\psi
\end{array}\right\}=\left[\begin{array}{ccc}
n_{1} & -n_{2} & 0 \\
n_{2} & n_{1} & 0 \\
0 & 0 & 1
\end{array}\right]\left\{\begin{array}{l}
u_{n} \\
u_{t} \\
\psi
\end{array}\right\}
$$

na qual $n_{1}$ e $n_{2}$ são as componentes do vetor normal. Seus valores podem ser obtidos a partir das coordenadas do elemento e das funções de forma através de:

$$
\begin{aligned}
& n_{1}(\xi)=\frac{1}{J(\xi)} \frac{\partial x_{2}(\xi)}{\partial \xi} \\
& n_{2}(\xi)=-\frac{1}{J(\xi)} \frac{\partial x_{1}(\xi)}{\partial \xi}
\end{aligned}
$$

onde $J(\xi)$ é o jacobiano dessa transformação, dado por: 


$$
J(\xi)=\sqrt{\left(\frac{\partial x_{1}(\xi)}{\partial \xi}\right)^{2}+\left(\frac{\partial x_{2}(\xi)}{\partial \xi}\right)^{2}}
$$

Aplicando a equação (4.14) em (4.13), fica:

$$
\begin{aligned}
\left\{\begin{array}{l}
u_{1_{0}} \\
u_{2_{0}}
\end{array}\right\} & =\left[\begin{array}{ccc}
\frac{1}{2}-\frac{\xi}{2} & 0 & n_{1}\left(\frac{\xi^{2}}{4}-\frac{1}{4}\right) \\
0 & \frac{1}{2}-\frac{\xi}{2} & n_{2}\left(\frac{\xi^{2}}{4}-\frac{1}{4}\right)
\end{array}\right]\left\{\begin{array}{l}
u_{1}^{1} \\
u_{2}^{1} \\
\psi^{1}
\end{array}\right\} \\
& +\left[\begin{array}{ccc}
\frac{1}{2}+\frac{\xi}{2} & 0 & -n_{1}\left(\frac{\xi^{2}}{4}-\frac{1}{4}\right) \\
0 & \frac{1}{2}+\frac{\xi}{2} & -n_{2}\left(\frac{\xi^{2}}{4}-\frac{1}{4}\right)
\end{array}\right]\left\{\begin{array}{c}
u_{n}^{2} \\
u_{t}^{2} \\
\psi^{2}
\end{array}\right\}
\end{aligned}
$$

O subscrito 0 foi acrescido para distinguir os deslocamentos obtidos desta forma das formulações convencionais. Definindo o seguinte termo:

$$
\hat{\xi}_{\alpha}=\frac{\xi-\xi_{\alpha}}{2}
$$

a equação (4.17) pode ser simplificada para:

$$
\begin{aligned}
\left\{\begin{array}{l}
u_{1_{0}} \\
u_{2_{0}}
\end{array}\right\} & =N_{1}^{c}(\xi)\left[\begin{array}{ccc}
1 & 0 & \hat{\xi}_{1}(\xi)\left(-n_{1}\right) \\
0 & 1 & \hat{\xi}_{1}(\xi)\left(-n_{2}\right)
\end{array}\right]\left\{\begin{array}{l}
u_{1}^{1} \\
u_{2}^{1} \\
\psi^{1}
\end{array}\right\} \\
& +N_{2}^{c}(\xi)\left[\begin{array}{ccc}
1 & 0 & \hat{\xi}_{2}(\xi)\left(-n_{1}\right) \\
0 & 1 & \hat{\xi}_{2}(\xi)\left(-n_{2}\right)
\end{array}\right]\left\{\begin{array}{l}
u_{n}^{2} \\
u_{t}^{2} \\
\psi^{2}
\end{array}\right\}
\end{aligned}
$$

onde os valores de $\xi_{i}$ são definidos de acordo com a posição dos nós de cada tipo de elemento, por exemplo para o elemento linear descontínuo temos que $\xi_{1}=-\frac{2}{3}$ e $\xi_{2}=\frac{2}{3}$. A nova função de forma (4.18) permite uma representação geral da equação (4.19) como:

$$
\left\{\begin{array}{l}
u_{1_{0}}^{e} \\
u_{2_{0}}^{e}
\end{array}\right\}=\sum_{\beta=1}^{2} N_{\beta}^{c}(\xi)\left[\begin{array}{ccc}
1 & 0 & \lambda \hat{\xi}_{\beta}(\xi)\left(-n_{1}\right) \\
0 & 1 & \lambda \hat{\xi}_{\beta}(\xi)\left(-n_{2}\right)
\end{array}\right]\left\{\begin{array}{l}
u_{1}^{\beta} \\
u_{2}^{\beta} \\
\psi^{\beta}
\end{array}\right\}
$$

onde o termo $\lambda$ é um valor arbitrário introduzido por [34] que pode ser utilizado para fazer com que as rotações se igualem àquelas da elasticidade. Cabe ressaltar que até o momento as rotações desenvolvidas nesta formulação não correspondem às teóricas. 


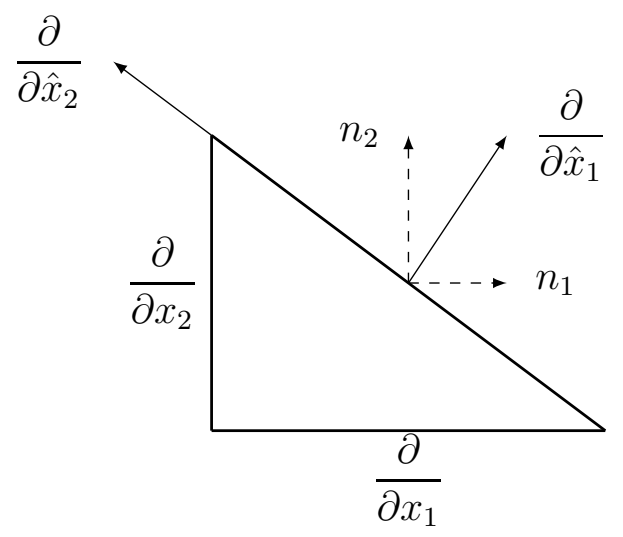

Figura 4.2: Relação entre derivadas no sistema local e global.

A equação (4.20) possui a forma de uma partição da unidade como indicado em [34]. Por conta disso, o processo descrito aqui, originalmente desenvolvido para um elemento finito, foi facilmente estendido ao método dos elementos de contorno e também para métodos sem malha.

O grau de liberdade rotacional dentro da formulação de elementos finitos foi originalmente incluído como uma maneira de incrementar a precisão do elemento, de tal maneira que não havia preocupação se a rotação calculada correspondia ou não à aquela da teoria clássica. No caso em que a ideia fosse modelar elementos para placas ou vigas, algumas considerações adicionais devem ser feitas para impor esta correspondência.

Primeiramente deve-se considerar o campo de rotações em torno do eixo $x_{3}$ dado pela teoria da elasticidade como apresentado na equação (2.10), repetida aqui por conveniência utilizando a notação apresentada em [25]:

$$
\omega_{3}=\Psi=\frac{1}{2}\left(u_{2,1}-u_{1,2}\right)
$$

A Figura 4.2 reproduzida de [25], ajuda a visualizar a transformação entre as derivadas locais e globais necessárias nos próximos passos. A relações são dadas por:

$$
\begin{aligned}
\frac{\partial}{\partial \hat{x}_{1}} & =n_{1} \frac{\partial}{\partial x_{1}}+n_{2} \frac{\partial}{\partial x_{2}} \\
\frac{\partial}{\partial \hat{x}_{2}} & =n_{1} \frac{\partial}{\partial x_{2}}-n_{2} \frac{\partial}{\partial x_{1}}
\end{aligned}
$$

e suas inversas são:

$$
\begin{aligned}
\frac{\partial}{\partial x_{1}} & =n_{1} \frac{\partial}{\partial \hat{x}_{1}}-n_{2} \frac{\partial}{\partial \hat{x}_{2}} \\
\frac{\partial}{\partial x_{2}} & =n_{1} \frac{\partial}{\partial \hat{x}_{2}}+n_{2} \frac{\partial}{\partial \hat{x}_{1}}
\end{aligned}
$$


Assim (2.10), pode ser expressa como:

$$
\begin{aligned}
\Psi & =\frac{1}{2}\left(\frac{\partial u_{2_{0}}}{\partial x_{1}}-\frac{\partial u_{1_{0}}}{\partial x_{2}}\right) \\
& =\frac{1}{2}\left[n_{1} \frac{\partial u_{2_{0}}}{\partial \hat{x}_{1}}-\frac{n_{2} \partial u_{2_{0}}}{\partial \hat{x}_{2}}-\left(n_{1} \frac{\partial u_{1_{0}}}{\partial \hat{x}_{1}}+n_{2} \frac{\partial u_{1_{0}}}{\partial \hat{x}_{2}}\right)\right]
\end{aligned}
$$

incluindo agora o campo de aproximação dos deslocamentos descritos com os conceitos de partição da unidade, encontra-se a forma discretizada da equação (4.23):

$$
\begin{aligned}
\Psi^{h} & =\frac{1}{2}\left[n_{1} \frac{\partial}{\partial \hat{x}_{1}}\left(N_{\beta} u_{2}^{\beta}-N_{\beta} \lambda \hat{\xi}_{\beta} n_{2} \psi^{\beta}\right)\right. \\
& -n_{2} \frac{\partial}{\partial \hat{x}_{2}}\left(N_{\beta} u_{2}^{\beta}-N_{\beta} \lambda \hat{\xi}_{\beta} n_{2} \psi^{\beta}\right) \\
& -n_{2} \frac{\partial}{\partial \hat{x}_{1}}\left(N_{\beta} u_{1}^{\beta}-N_{\beta} \lambda \hat{\xi}_{\beta} n_{1} \psi^{\beta}\right) \\
& \left.-n_{1} \frac{\partial}{\partial \hat{x}_{2}}\left(N_{\beta} u_{1}^{\beta}-N_{\beta} \lambda \hat{\xi}_{\beta} n_{1} \psi^{\beta}\right)\right]
\end{aligned}
$$

Levando em consideração agora que as normais são constantes para elementos lineares, a equação (4.24) pode ser escrita como:

$$
\begin{aligned}
\Psi^{h} & =\frac{1}{2}\left[\frac{\partial}{\partial \hat{x}_{1}}\left(n_{1} N_{\beta} u_{2}^{\beta}-n_{2} N_{\beta} u_{1}^{\beta}\right)\right. \\
& -\frac{\partial}{\partial \hat{x}_{2}}\left(n_{2} N_{\beta} u_{2}^{\beta}-n_{1} N_{\beta} u_{1}^{\beta}\right) \\
& +\frac{\partial}{\partial \hat{x}_{2}}\left(-n_{1} n_{2} N_{\beta} \lambda \hat{\xi}_{\beta} \psi^{\beta}+n_{1} n_{2} N_{\beta} \lambda \hat{\xi}_{\beta} \psi^{\beta}\right) \\
& \left.+\frac{\partial}{\partial \hat{x}_{2}}\left(n_{2}^{2} N_{\beta} \lambda \hat{\xi}_{\beta} \psi^{\beta}+n_{1}^{2} N_{\beta} \lambda \hat{\xi}_{\beta} \psi^{\beta}\right)\right]
\end{aligned}
$$

A expressão (4.25), considerando que a terceira linha se anula e que $n_{1}^{2}+n_{2}^{2}=1$, se reduz a:

$$
\Psi^{h}=\Psi^{\text {elem }}+\frac{\lambda}{2}\left(N_{1} \frac{\partial \hat{\xi}_{1}}{\partial \hat{x}_{2}}+\hat{\xi}_{1} \frac{\partial N_{1}}{\partial \hat{x}_{2}}\right) \psi^{1}+\frac{\lambda}{2}\left(N_{2} \frac{\partial \hat{\xi}_{2}}{\partial \hat{x}_{2}}+\hat{\xi}_{2} \frac{\partial N_{2}}{\partial \hat{x}_{2}}\right) \psi^{2}
$$

onde $\Psi^{\text {elem }}$ é a rotação do elemento, baseado nos deslocamentos no sistema local [33] dado por:

$$
\Psi^{e l e m}=\frac{1}{2}\left(\frac{\partial u_{t}}{\partial \hat{x}_{1}}-\frac{\partial u_{n}}{\partial \hat{x}_{2}}\right)
$$

A equação (4.26) pode então ser expressa como: 


$$
\begin{aligned}
\Psi^{h} & =\Psi^{e l e m}+\frac{\lambda}{2}\left(N_{1}(\xi) \frac{d \xi}{d \hat{x}_{2}} \frac{d \hat{\xi}_{1}(\xi)}{d \xi}+\hat{\xi}_{1}(\xi) \frac{d \xi}{d \hat{x}_{2}} \frac{d N_{1}(\xi)}{d \xi}\right) \psi^{1} \\
& +\frac{\lambda}{2}\left(N_{2}(\xi) \frac{d \xi}{d \hat{x}_{2}} \frac{d \hat{\xi}_{2}(\xi)}{d \xi}+\hat{\xi}_{2}(\xi) \frac{d \xi}{d \hat{x}_{2}} \frac{d N_{2}(\xi)}{d \xi}\right) \psi^{2}
\end{aligned}
$$

na qual a coordenada local $\hat{\xi}_{2}$ é relacionada à paramétrica por [12]:

$$
\frac{d \xi}{d \hat{x}_{2}}=\frac{1}{J(\xi)}
$$

Em [25], Leung \& Baiz (2013) comentam que os termos contendo coordenadas normais locais $\hat{x}_{1}$ que multiplicam $w^{\alpha}$ desaparecem, o que reforça as suposições apresentadas em [27], que relacionam as rotações às derivadas nas direções tangenciais.

Especificando a equação (4.28) para os nós, tem-se que:

$$
\Psi^{\alpha}=\Psi^{e l e m}+\frac{\lambda}{4 J(\xi)}\left(2 \psi^{\alpha}-\psi^{1}-\psi^{2}\right) \quad \alpha=1,2 .
$$

a qual é uma expressão similar àquela apresentada em [33]. Desta equação pode-se concluir que $\psi^{\alpha}$ não correspondem exatamente às rotações teóricas mas possuem uma estreita relação. Leung \& Baiz (2013) [25] observam que para haver essa correspondência, é necessário que $\lambda=2 J(\xi)$ e ao mesmo tempo,

$$
\begin{gathered}
\Psi^{\text {elem }}=\frac{\psi^{1}+\psi^{2}}{2} \\
\frac{1}{2}\left(\frac{\partial u_{t}}{\partial \hat{x}_{1}}-\frac{\partial u_{n}}{\partial \hat{x}_{2}}\right)-\frac{\psi^{1}+\psi^{2}}{2}=0
\end{gathered}
$$

\subsubsection{Relacão entre as rotações e a rotação da teoria da elasticidade.}

Por conta da inclusão da rotação em torno do eixo $x_{3}$ na formulação, é necessário incluir mais uma relação para cada nó de maneira que o sistema de equações formado possa ser resolvido. Essas relações podem ser obtidas criando um funcional que é, ao mesmo tempo, usado para impor que as rotações calculadas sejam aquelas da teoria da elasticidade. Assim, definindo o seguinte funcional, temos:

$$
\Pi\left(u_{i}, \bar{\psi}\right)=\frac{\gamma}{2} \int_{\Gamma}\left(\psi^{e l e m}-\bar{\psi}\right)^{2} d \Gamma
$$

onde o termo $\psi^{\text {elem }}$ é o campo de rotações verdadeiro e $\gamma$ é um parâmetro de penalidade que, neste caso, não tem influência sobre a solução do problema. Este parâmetro se torna importante apenas quando usado com o funcional da energia potencial total, 
para balancear sua contribuição ao problema bidimensional clássico. Para incluir esta equação no sistema, deve-se alterá-la para sua forma discretizada, que fica:

$$
\begin{aligned}
\Psi_{\text {elem }}^{h}= & \frac{1}{2}\left[\frac{\partial N_{1}(\xi)}{\partial \hat{x}_{2}}\left(-u_{1}^{1} n_{1}-u_{2}^{1} n_{2}\right)+\frac{\partial N_{2}(\xi)}{\partial \hat{x}_{2}}\left(-u_{1}^{2} n_{1}-u_{2}^{2} n_{2}\right)\right. \\
& \left.+\frac{\partial N_{1}(\xi)}{\partial \hat{x}_{2}}\left(-u_{1_{0}}^{1} n_{1}-u_{2_{0}}^{1} n_{2}\right)+\frac{\partial N_{2}(\xi)}{\partial \hat{x}_{2}}\left(-u_{1_{0}}^{2} n_{1}-u_{2_{0}}^{2} n_{2}\right)\right]
\end{aligned}
$$

que pode ser reduzido para:

$$
\begin{aligned}
\Psi_{\text {elem }}^{h} & =\frac{1}{2 J(\xi)}\left[\frac{d N_{1}(\xi)}{d \xi}\left(-u_{1}^{1} n_{1}-u_{2}^{1} n_{2}\right)+\frac{d N_{2}(\xi)}{d \xi}\left(-u_{1}^{2} n_{1}-u_{2}^{2} n_{2}\right)\right] \\
& =[\mathbf{B}]_{1 \times 4}\left[\mathbf{d}_{\mathbf{0}}\right]_{4 \times 1}
\end{aligned}
$$

onde os termos que continham derivadas em relação à direção normal foram ignorados, uma vez que não estão definidas para o elemento de contorno [25]. A explicação para isso é que a direção normal não é diretamente descrita em função da variável paramétrica $\xi$.

De maneira análoga o campo de rotações reais em sua forma discretizada é dado por:

$$
\bar{\psi}^{h}=\frac{1}{2}\left[N_{1}(\xi) \psi^{1}+N_{2}(\xi) \psi^{2}\right]=[\mathbf{N}]_{1 \times 2}\left[\mathbf{d}_{\psi}\right]_{2 \times 1}
$$

Tomando o primeiro variacional da equação (4.33) em relação ao campo de rotações e igualando-o a zero com o intuito de minimizar o funcional, obtém-se:

$$
\frac{\delta \Pi(u, \bar{\psi})}{\delta \bar{\psi}}=\int_{\Gamma} \delta \bar{\psi}^{h T}\left(\psi_{0}^{h}-\bar{\psi}^{h}\right) d \Gamma=0
$$

Após substituir a forma discretizada de $\bar{\psi}^{h}$ e $\psi_{0}^{h}$ e transformando também para o domínio paramétrico do elemento tem-se que:

$$
\left[\delta \mathbf{d}_{\psi}\right]_{2 \times 1}^{T} \int_{-1}^{+1}\left[\mathbf{K}_{\psi \times 0 \psi}\right]_{2 \times 6} J^{m}(\xi) d \xi\left[\delta \mathbf{d}_{0_{\psi}}^{m}\right]_{6 \times 1}=\mathbf{0}
$$

onde

e

$$
\begin{aligned}
& {\left[\mathbf{K}_{\mathbf{w} \times \mathbf{0 w}}\right]=\left[\begin{array}{lll}
N_{1}(\xi) \frac{n_{1}}{J(\xi)} \frac{d N_{1}(\xi)}{d \xi} & N_{1}(\xi) \frac{n_{2}}{J(\xi)} \frac{d N_{1}(\xi)}{d \xi} & N_{1}(\xi) N_{1}(\xi) \\
N_{2}(\xi) \frac{n_{1}}{J(\xi)} \frac{d N_{1}(\xi)}{d \xi} & N_{2}(\xi) \frac{n_{2}}{J(\xi)} \frac{d N_{1}(\xi)}{d \xi} & N_{2}(\xi) N_{1}(\xi)
\end{array}\right.} \\
& \left.\begin{array}{lll}
N_{1}(\xi) \frac{n_{1}}{J(\xi)} \frac{d N_{2}(\xi)}{d \xi} & N_{1}(\xi) \frac{n_{2}}{J(\xi)} \frac{d N_{2}(\xi)}{d \xi} & N_{1}(\xi) N_{2}(\xi) \\
N_{2}(\xi) \frac{n_{1}}{J(\xi)} \frac{d N_{2}(\xi)}{d \xi} & N_{2}(\xi) \frac{n_{2}}{J(\xi)} \frac{d N_{2}(\xi)}{d \xi} & N_{2}(\xi) N_{2}(\xi)
\end{array}\right]
\end{aligned}
$$

$$
\left[\delta \mathbf{d}_{\mathbf{0} \psi}{ }^{m}\right]_{6 \times 1}^{T}=\left[\begin{array}{llllll}
u_{1_{0}}^{1} & u_{2_{0}}^{1} & \psi^{1} & u_{1_{0}}^{2} & u_{2_{0}}^{2} & \psi^{2}
\end{array}\right]
$$

Estas equações são usadas apenas no elemento onde está o ponto fonte e cada uma das linhas é incluída na linha correspondente à rotação do nó no elemento. 


\subsubsection{Equações integrais com elementos de contorno}

A equação integral de contorno para elasticidade plana foi derivada no capítulo 3 e é dada pela expressão (3.37). Após o extenso desenvolvimento apresentado na seção anterior, o sistema formado por esta equação se altera. É feita a inclusão de rotações como variáveis nodais, da forma fraca do resíduo do campo de rotações para o elemento (4.38) e o acréscimo das funções de forma enriquecidas (4.18). Considerando todas estas alterações, a equação integral de contorno se torna:

$$
\begin{aligned}
& {\left[\begin{array}{ccc}
C_{11}^{c} & C_{12}^{c} & 0 \\
C_{21}^{c} & C_{22}^{c} & 0 \\
0 & 0 & 1
\end{array}\right]\left\{\begin{array}{l}
u_{1_{0}}^{c 1} \\
u_{2_{0}}^{c 1} \\
\omega^{c 1}
\end{array}\right\}+\left[\begin{array}{ccc}
H_{11}^{* m c 1} & H_{12}^{* m c 1} & H_{13}^{* m c 1} \\
H_{21}^{* m c 1} & H_{22}^{* m} c 1 & H_{23}^{* m c 1} \\
K_{\alpha 1} & K_{\alpha 2} & K_{\alpha 3}
\end{array}\right]\left\{\begin{array}{l}
u_{1_{0}}^{c 1} \\
u_{2_{0}}^{c 1} \\
\omega^{c 1}
\end{array}\right\}+} \\
& {\left[\begin{array}{ccc}
H_{11}^{* m c 2} & H_{12}^{* m c 2} & H_{13}^{* m c 2} \\
H_{21}^{* m c 2} & H_{22}^{* m c 2} & H_{23}^{* m c 2} \\
K_{\alpha 4} & K_{\alpha 5} & K_{\alpha 6}
\end{array}\right]\left\{\begin{array}{l}
u_{1_{0}}^{c 2} \\
u_{2_{0}}^{c 2} \\
\omega^{c 2}
\end{array}\right\}+\sum_{m=1, m \neq c}^{N e} \sum_{\beta=1}^{2}\left[\begin{array}{ccc}
H_{11}^{* m \beta} & H_{12}^{* m \beta} & H_{13}^{* m \beta} \\
H_{21}^{* m \beta} & H_{22}^{* m \beta} & H_{23}^{* m \beta} \\
0 & 0 & 0
\end{array}\right]\left\{\begin{array}{l}
u_{1_{0}}^{m \beta} \\
u_{2_{0}}^{m \beta} \\
\omega^{m \beta}
\end{array}\right\}} \\
& =\sum_{m=1}^{N e} \sum_{\beta=1}^{2}\left[\begin{array}{ccc}
G_{11}^{m \beta} & G_{12}^{m \beta} & 0 \\
G_{21}^{m \beta} & G_{22}^{m \beta} & 0 \\
0 & 0 & 1
\end{array}\right]\left\{\begin{array}{c}
t_{1}^{m \beta} \\
t_{2}^{m \beta} \\
0
\end{array}\right\}
\end{aligned}
$$

onde $c$ é ponto fonte contido no elemento $m$, o sobre escrito $\beta$ se refere à numeração do nó no elemento sendo integrado. Para um caso linear pode assumir valores 1 e 2 . O sub escrito $\alpha$ assume valores 1 ou 2 de acordo com a posição do ponto fonte no elemento. A matriz $G_{i j}^{m \beta}$ é inalterada nesta formulação em relação à convencional, os termos $H_{i j}^{* m \beta}$, contudo são obtidos a partir da seguinte integral:

$$
\begin{aligned}
{\left[\begin{array}{lll}
H_{11}^{* m \beta} & H_{12}^{* m \beta} & H_{13}^{* m \beta} \\
H_{21}^{* m \beta} & H_{22}^{* m \beta} & H_{23}^{* m \beta}
\end{array}\right]=} & \int_{-1}^{+1}\left[\begin{array}{cc}
T_{11}^{*}\left(\mathbf{x}^{\prime}, \mathbf{x}\right) & T_{12}^{*}\left(\mathbf{x}^{\prime}, \mathbf{x}\right) \\
T_{21}^{*}\left(\mathbf{x}^{\prime}, \mathbf{x}\right) & T_{22}^{*}\left(\mathbf{x}^{\prime}, \mathbf{x}\right)
\end{array}\right] \\
& \times\left\{N_{\beta}\left[\begin{array}{ccc}
1 & 0 & -n_{1} \lambda \hat{\xi}_{\beta} \\
0 & 1 & -n_{2} \lambda \hat{\xi}_{\beta}
\end{array}\right]\right\} J(\xi) d \xi
\end{aligned}
$$

\subsection{Equação integral de contorno para rotação no plano}

A principal contribuição deste trabalho se origina da derivação de uma expressão analítica para o cálculo da rotação em torno do eixo $x_{3}$. A equação (3.37) dá valores para deslocamento em pontos de colocação específicos, os pontos fonte, que podem ser posicionados em qualquer local. Como mostrado na Seção 2.2 as derivadas da expressão do deslocamento precisam ser calculadas para que seja possível encontrar um resultado para a equação (2.10). Neste caso, as derivadas são calculadas em relação ao ponto 
fonte e, levando em consideração que apenas as soluções fundamentais são função desta variável, a expressão para a derivada da equação (3.37) em relação a x' é dada pela equação (3.18) no qual o ponto fonte foi levado ao contorno:

$$
u_{\alpha, \gamma}\left(\mathbf{x}^{\prime}\right)=2\left[f_{\Gamma} U_{\alpha \beta, \gamma}^{*}\left(\mathbf{x}^{\prime}, \mathbf{x}\right) t_{\beta}(\mathbf{x}) d \Gamma(\mathbf{x})-f_{\Gamma} T_{\alpha \beta, \gamma}^{*}\left(\mathbf{x}^{\prime}, \mathbf{x}\right) u_{\beta}(\mathbf{x}) d \Gamma(\mathbf{x})\right]
$$

onde o símbolo f representa uma integração no sentido do valor principal de Hadamard [12]. As derivadas das soluções fundamentais de deslocamento e forças de superfície foram apresentadas na igualdade (4.43), e são dadas pelas equações (3.32) e (3.33). Substituindo (4.43) na expressão (2.10), chega-se a equação integral de contorno para a rotação em torno do eixo $x_{3}$ :

$$
\begin{gathered}
\omega_{3}\left(\mathbf{x}^{\prime}\right)=-f_{\Gamma}\left(T_{2 \beta, 1}^{*}\left(\mathbf{x}^{\prime}, \mathbf{x}\right)-T_{1 \beta, 2}^{*}\left(\mathbf{x}^{\prime}, \mathbf{x}\right)\right) u_{\beta}(\mathbf{x}) d \Gamma(\mathbf{x}) \\
+f_{\Gamma}\left(U_{2 \beta, 1}^{*}\left(\mathbf{x}^{\prime}, \mathbf{x}\right)-U_{1 \beta, 2}^{*}\left(\mathbf{x}^{\prime}, \mathbf{x}\right)\right) t_{\beta}(\mathbf{x}) d \Gamma(\mathbf{x})
\end{gathered}
$$

Analisando a equação (4.44) podem ser observadas algumas características. Primeiramente, trata-se de uma expressão analítica para a obtenção da rotação em torno do eixo $x_{3}$, aproximações serão feitas apenas quando o contorno do problema for discretizado. A rotação depende diretamente das variáveis $u_{\alpha}$ e $t_{\alpha}$ e não necessita de um $w_{3}$ nodal. É portanto uma resposta secundária, assim como as tensões mas que pode ser calculada paralelamente no sistema de equações original.

Em sessões posteriores será mostrado como a rotação calculada através da elasticidade plana influenciará nas rotações das formulações de placas. Basicamente esta rotação pode mudar de orientação quando da imposição de condições de compatibilidade entre placas localizadas em diferentes planos.

\subsubsection{Equação integral de contorno para rotação no plano com elementos de contorno}

Combinando as equações (3.37) e (4.44), obtém-se:

$$
\begin{gathered}
{\left[\begin{array}{ccc}
C_{11}^{s} & C_{12}^{s} & 0 \\
C_{21}^{s} & C_{22}^{s} & 0 \\
0 & 0 & 1
\end{array}\right]\left\{\begin{array}{c}
u_{1}^{s 1} \\
u_{2}^{s 1} \\
w_{3}^{s 1}
\end{array}\right\}+\sum_{m=1}^{N e} \sum_{\eta=1}^{n n o s}\left[\begin{array}{ccc}
H_{11}^{m \eta} & H_{12}^{m \eta} & 0 \\
H_{21}^{m \eta} & H_{22}^{m \eta} & 0 \\
H_{31}^{m \eta} & H_{32}^{m \eta} & 0
\end{array}\right]\left\{\begin{array}{c}
u_{1}^{m \eta} \\
u_{2}^{m \eta} \\
0
\end{array}\right\}} \\
=\sum_{m=1}^{N e} \sum_{\eta=1}^{n n o s}\left[\begin{array}{lll}
G_{11}^{m \eta} & G_{12}^{m \eta} & 0 \\
G_{21}^{m \eta} & G_{22}^{m \eta} & 0 \\
G_{31}^{m \eta} & G_{32}^{m \eta} & 0
\end{array}\right]\left\{\begin{array}{c}
t_{1}^{m \eta} \\
t_{2}^{m \eta} \\
0
\end{array}\right\}
\end{gathered}
$$


para a formulação de elasticidade plana modificada, onde $N e$ é o número de elementos no problema, nnos é o número de nós por elemento, $s$ é o ponto fonte. Os termos das novas matrizes de influência são dados por:

$$
\begin{aligned}
H_{\alpha \beta}^{m \eta} & =f_{-1}^{1} T_{\alpha \beta}^{*} \Phi_{\eta} J(\xi) d \xi \\
G_{\alpha \beta}^{m \eta} & =f_{-1}^{1} U_{\alpha \beta}^{*} \Phi_{\eta} J(\xi) d \xi \\
H_{3 \beta}^{m \eta} & =f_{-1}^{1} N_{\eta}^{d}\left(T_{2 \beta, 1}^{*}-T_{1 \beta, 2}^{*}\right) J(\xi) d \xi \\
G_{3 \beta}^{m \eta} & =f_{-1}^{1} N_{\eta}^{d}\left(U_{2 \beta, 1}^{*}-U_{1 \beta, 2}^{*}\right) J(\xi) d \xi
\end{aligned}
$$

A matriz de funções de forma para o caso de elementos de contorno lineares é dada por:

$$
\Phi_{\eta}=\left[\begin{array}{cc}
N_{\eta}^{d} & 0 \\
0 & N_{\eta}^{d}
\end{array}\right]
$$

e para o caso de elementos quadráticos:

$$
\Phi_{\eta}=\left[\begin{array}{ccc}
N_{\eta}^{d} & 0 & 0 \\
0 & N_{\eta}^{d} & 0 \\
0 & 0 & N_{\eta}^{d}
\end{array}\right]
$$

Neste ponto, a formulação aqui apresentada difere da convencional pela presença dos termos (4.48) e (4.49), relacionadas com a equação recém desenvolvida para a rotação em torno do eixo $x_{3}$. Portanto, as novas matrizes de influência elementares para elasticidade plana são:

e

$$
H^{s}=\left[\begin{array}{lll}
H_{11}^{m \eta} & H_{12}^{m \eta} & 0 \\
H_{21}^{m \eta} & H_{22}^{m \eta} & 0 \\
H_{31}^{m \eta} & H_{32}^{m \eta} & 0
\end{array}\right]
$$

$$
G^{s}=\left[\begin{array}{ccc}
G_{11}^{m \eta} & G_{12}^{m \eta} & 0 \\
G_{21}^{m \eta} & G_{22}^{m \eta} & 0 \\
G_{31}^{m \eta} & G_{32}^{m \eta} & 0
\end{array}\right]
$$

O desenvolvimento necessário para a implementação e resolução do sistema foi apresentado. Convém ressaltar que todo a manipulação matemática independe da função de forma utilizada no problema, ao contrário do que acontece na formulação apresentada por [25], cuja derivação foi bastante simplificada pelo grau da função de 
forma, se beneficiando também do fato da normal ser constante ao longo do elemento. Todas as integrais apresentadas podem ser resolvidas utilizando a quadratura gaussiana sempre que o ponto fonte não pertencer ao elemento sendo integrado. Nos casos em que isto ocorrer, considerações de corpo rígido serão usada para resolver as integrais da formulação original. Os termos que foram acrescentados possuem singularidades e serão tratados da maneira explicada a seguir.

\subsection{Tratamento de singularidades}

Quando o ponto fonte pertence ao elemento que está sendo integrado, surgem dois tipos de singularidades nas expressões (4.48) e (4.49). Aquela envolvendo o termo $T_{\alpha \beta, \gamma}^{*}$ é fortemente singular de ordem $\mathrm{O}\left(r^{-2}\right)$ e aquela envolvendo o termo $U_{\alpha \beta, \gamma}^{*}$ é fracamente singular de ordem $\mathrm{O}\left(r^{-1}\right)$. A abordagem utilizada neste trabalho é a mesma apresentada em [44]. Existem duas maneiras principais de tratamento para estas integrais, a subtração de singularidades [45] e sua interpretação no sentido dos valores de Cauchy e Hadamard, calculando diretamente a parte finita destas integrais. Especificando para o caso de elementos retilíneos, nos quais o Jacobiano e as normais são constantes ao longo do elemento, o tratamento destas integrais pode ser feito analiticamente. Considere novamente as expressões (4.48) e (4.49). Os termos subtraídos podem ser separados da seguinte maneira:

$$
\begin{aligned}
H_{3 \beta}^{m \eta} & =f_{-1}^{1} T_{2 \beta, 1}^{*} N_{\eta}^{d} J(\xi) d \xi-f_{-1}^{1} T_{1 \beta, 2}^{*} N_{\eta}^{d} J(\xi) d \xi \\
G_{3 \beta}^{m \eta} & =f_{-1}^{1} U_{2 \beta, 1}^{*} N_{\eta}^{d} J(\xi) d \xi-f_{-1}^{1} U_{1 \beta, 2}^{*} N_{\eta}^{d} J(\xi) d \xi
\end{aligned}
$$

É possível realizar então as seguintes operações para cada um dos dois tipos de integral:

$$
I_{1}=f_{-1}^{1} T_{\alpha \beta, \gamma}^{*} N_{\eta}^{d}(\xi) J(\xi) d \xi=F_{\alpha \beta \gamma}^{*} f_{-1}^{1} \frac{N_{\eta}^{d}(\xi)}{\left(\xi-\xi^{\prime}\right)^{2}} d \xi
$$

onde

$$
F_{\alpha \beta \gamma}^{*}=T_{\alpha \beta, \gamma}^{*} J(\xi)\left(\xi-\xi^{\prime}\right)^{2}
$$

e

$$
I_{2}=f_{-1}^{1} U_{\alpha \beta, \gamma}^{*} N_{\eta}^{d}(\xi) J(\xi) d \xi=S_{\alpha \beta \gamma}^{*} \int_{-1}^{1} \frac{N_{\eta}^{d}(\xi)}{\xi-\xi^{\prime}} d \xi
$$

onde 


$$
S_{\alpha \beta \gamma}^{*}=U_{\alpha \beta, \gamma}^{*} J(\xi)\left(\xi-\xi^{\prime}\right)
$$

Os termos $F_{\alpha \beta \gamma}^{*}$ e $S_{\alpha \beta \gamma}^{*}$ são constantes e, por isso, podem ser retirados das integrais. Os resultados para os termos remanescentes podem ser calculados analiticamente e são dados pelas equações (4.58) a (4.61) dependendo agora apenas do ponto fonte $\xi_{0}$ no sistema de coordenadas intrínsecos. As respostas analíticas são apresentadas abaixo.

$$
\begin{gathered}
\int_{-1}^{1} \frac{N_{1}^{d}}{\xi-\xi_{0}} d \xi=\frac{1}{4}\left(\left(2-3 \xi_{0}\right) \ln \left(4-4 \xi_{0}\right)+\left(-2+3 \xi_{0}\right) \ln \left(-4\left(1+\xi_{0}\right)\right)-6\right) \\
\int_{-1}^{1} \frac{N_{2}^{d}}{\xi-\xi_{0}} d \xi=\frac{1}{4}\left(\left(2+3 \xi_{0}\right) \ln \left(4-4 \xi_{0}\right)-\left(2+3 \xi_{0}\right) \ln \left(-4\left(1+\xi_{0}\right)\right)+6\right) \\
\int_{-1}^{1} \frac{N_{1}^{d}}{\left(\xi-\xi_{0}\right)^{2}} d \xi=\frac{4-6 \xi_{0}+3\left(-1+\xi_{0}^{2}\right) \ln \left(-1-\xi_{0}\right)-3\left(-1+\xi_{0}^{2}\right) \ln \left(1-\xi_{0}\right)}{4\left(-1+\xi_{0}^{2}\right)} \\
\int_{-1}^{1} \frac{N_{2}^{d}}{\left(\xi-\xi_{0}\right)^{2}} d \xi=\frac{4+6 \xi_{0}-3\left(-1+\xi_{0}^{2}\right) \ln \left(-1-\xi_{0}\right)+3\left(-1+\xi_{0}^{2}\right) \ln \left(1-\xi_{0}\right)}{4\left(-1+\xi_{0}^{2}\right)}
\end{gathered}
$$

Elementos lineares serão retilíneos independente do problema a ser tratado. Os elementos quadráticos contudo, podem descrever curvas parabólicas, o que inviabiliza a abordagem discutida acima, uma vez que as normais e o jacobiano deixam de ser constantes. Assim a formulação aqui apresentada é aplicável à classe de problemas cuja geometria a ser tratada, envolve apenas segmentos formados por linhas retas. Os resultados para elementos quadráticos, considerando estas restrições, são dados por:

$$
\begin{gathered}
\int_{-1}^{1} \frac{N_{1}^{d}}{\xi-\xi_{0}} d \xi=-\frac{3}{8}\left(3 \xi_{0}-2\right)\left(\xi_{0} \ln \left(-\xi_{0}-1\right)-\xi_{0} \ln \left(1-\xi_{0}\right)-2\right) \\
\int_{-1}^{1} \frac{N_{2}^{d}}{\xi-\xi_{0}} d \xi=\left(1-\frac{9 \xi_{0}^{2}}{4}\right) \ln \left(4-4 \xi_{0}\right)+\left(\frac{9 \xi_{0}^{2}}{4}-1\right) \ln \left(-4\left(\xi_{0}+1\right)\right)-\frac{9 \xi_{0}}{2} \\
\int_{-1}^{1} \frac{N_{3}^{d}}{\xi-\xi_{0}} d \xi=-\frac{3}{8}\left(3 \xi_{0}+2\right)\left(\xi_{0} \ln \left(-\xi_{0}-1\right)-\xi_{0} \ln \left(1-\xi_{0}\right)-2\right) \\
\int_{-1}^{1} \frac{N_{1}^{d}}{\left(\xi-\xi_{0}\right)^{2}} d \xi=\frac{3}{8}\left(\frac{1}{\xi_{0}-1}+\left(2-6 \xi_{0}\right) \ln \left(-\xi_{0}-1\right)\right. \\
\left.-\frac{5}{\xi_{0}+1}+\left(6 \xi_{0}-2\right) \ln \left(1-\xi_{0}\right)+12\right)
\end{gathered}
$$




$$
\begin{array}{r}
\int_{-1}^{1} \frac{N_{2}^{d}}{\left(\xi-\xi_{0}\right)^{2}} d \xi=\frac{-18 \xi_{0}^{2}+9\left(\xi_{0}^{2}-1\right) \xi_{0}\left(\ln \left(-\xi_{0}-1\right)-\ln \left(1-\xi_{0}\right)\right)+13}{2\left(\xi_{0}^{2}-1\right)} \\
\int_{-1}^{1} \frac{N_{3}^{d}}{\left(\xi-\xi_{0}\right)^{2}} d \xi=\frac{3}{8}\left(\frac{5}{\xi_{0}-1}-2\left(3 \xi_{0}+1\right) \ln \left(-\xi_{0}-1\right)\right. \\
\left.\quad-\frac{1}{\xi_{0}+1}+\left(6 \xi_{0}+2\right) \ln \left(1-\xi_{0}\right)+12\right)
\end{array}
$$




\section{FORMULAÇÃO ACOPLADA PARA ASSOCIAÇÃO DE PLACAS E ASPECTOS COMPUTACIONAIS}

\subsection{Introdução}

Neste capítulo, os aspectos finais da implementação serão abordados. Até o momento as duas formulações, de membrana e placas deformáveis por cisalhamento, foram apresentadas paralelamente ao longo do capítulo 3. A contribuição deste trabalho com a equação integral de contorno para a rotação $\omega_{3}$, assim como a formulação desenvolvida por [25] foram obtidas e apresentadas por todo o capítulo 4. As seções a seguir, tem a intenção de agrupar estas formulações em uma só e dar os detalhes finais a respeito da implementação da formulação para associação de placas no espaço.

\subsection{Associação de Placas Espessas}

Este trabalho trata da associação de placas utilizando o método dos elementos de contorno. Como explicado anteriormente, a abordagem considera a combinação das formulações capturando os comportamentos fletores e extensores de uma placa.

Para cada um dos pontos de colocação, tem-se agora 6 graus de liberdade, as equações (3.37), (4.44) e (3.54) podem ser combinadas, de tal maneira a resultar no seguinte conjunto de equações para um sistema local, escrita na forma matricial:

$$
\left[\begin{array}{cc}
\mathbf{H}^{\mathbf{p}} & \mathbf{0} \\
\mathbf{0} & \mathbf{H}^{\mathrm{ep}}
\end{array}\right]_{6 \times 6}\left\{\begin{array}{l}
\mathbf{w} \\
\mathbf{u}
\end{array}\right\}_{6 \times 1}=\left[\begin{array}{cc}
\mathbf{G}^{\mathbf{p}} & \mathbf{0} \\
\mathbf{0} & \mathbf{G}^{\mathbf{e p}}
\end{array}\right]_{6 \times 6}\left\{\begin{array}{l}
\mathbf{p} \\
\mathbf{t}
\end{array}\right\}_{6 \times 1}+\left\{\begin{array}{l}
\mathbf{b} \\
\mathbf{0}
\end{array}\right\}_{6 \times 1}
$$

onde

$$
\mathbf{u}=\left\{\begin{array}{l}
u_{1} \\
u_{2} \\
w_{3}
\end{array}\right\} \quad \mathbf{w}=\left\{\begin{array}{l}
w_{1} \\
w_{2} \\
u_{3}
\end{array}\right\}
$$

são os deslocamentos generalizados,

$$
\mathbf{t}=\left\{\begin{array}{c}
t_{1} \\
t_{2} \\
0
\end{array}\right\} \quad \mathbf{p}=\left\{\begin{array}{c}
m_{1} \\
m_{2} \\
t_{3}
\end{array}\right\}
$$


são as forças de superfície generalizadas e b é o vetor cargas de domínio oriundo das formulações de placa. Os termos $\mathbf{H}^{\mathbf{p}}, \mathbf{G}^{\mathbf{p}}, \mathbf{H}^{\mathrm{ep}}$ e $\mathbf{G}^{\mathbf{e p}}$ são as matrizes de influência das formulações de flexão de placas ()$^{p}$ e elasticidade plana modificada ()$^{e p}$, como abordado em [3].

As funções de forma descontínuas (3.60) e (3.62) são utilizadas para aproximar os deslocamentos generalizados e forças de superfície ao longo do elemento. Assim, para a formulação acoplada, expandindo a equação (5.1) tem-se:

$$
\begin{aligned}
& {\left[\begin{array}{cccccc}
C_{11}^{p} & C_{12}^{p} & C_{13}^{p} & 0 & 0 & 0 \\
C_{21}^{p} & C_{22}^{p} & C_{23}^{p} & 0 & 0 & 0 \\
C_{31}^{p} & C_{32}^{p} & C_{33}^{p} & 0 & 0 & 0 \\
0 & 0 & 0 & C_{11}^{e p} & C_{12}^{e p} & 0 \\
0 & 0 & 0 & C_{21}^{e p} & C_{22}^{e p} & 0 \\
0 & 0 & 0 & 0 & 0 & 1
\end{array}\right]\left\{\begin{array}{c}
\omega_{1}^{s 1} \\
\omega_{2}^{s 1} \\
u_{3}^{s 1} \\
u_{1}^{s 1} \\
u_{2}^{s 1} \\
\omega_{3}^{s 1}
\end{array}\right\}+} \\
& \sum_{m=1}^{N e} \sum_{\eta=1}^{n n o s} \int_{-1}^{1} \Phi\left[\begin{array}{cccccc}
P_{11}^{m \eta} & P_{12}^{m \eta} & P_{13}^{m \eta} & 0 & 0 & 0 \\
P_{21}^{m \eta} & P_{22}^{m \eta} & P_{23}^{m \eta} & 0 & 0 & 0 \\
P_{31}^{m \eta} & P_{32}^{m \eta} & P_{33}^{m \eta} & 0 & 0 & 0 \\
0 & 0 & 0 & U_{11}^{m \eta} & U_{12}^{m \eta} & 0 \\
0 & 0 & 0 & U_{21}^{m \eta} & U_{22}^{m \eta} & 0 \\
0 & 0 & 0 & U_{31}^{m \eta} & U_{32}^{m \eta} & 0
\end{array}\right] J(\xi) d \xi\left\{\begin{array}{c}
\omega_{1}^{m \eta} \\
\omega_{2}^{m \eta} \\
u_{3}^{m \eta} \\
u_{1}^{m \eta} \\
u_{2}^{m \eta} \\
0
\end{array}\right\} \\
& =\sum_{m=1}^{N e} \sum_{\eta=1}^{n n o s} \int_{-1}^{1} \Phi\left[\begin{array}{cccccc}
W_{11}^{m \eta} & W_{12}^{m \eta} & W_{13}^{m \eta} & 0 & 0 & 0 \\
W_{21}^{m \eta} & W_{22}^{m \eta} & W_{23}^{m \eta} & 0 & 0 & 0 \\
W_{31}^{m \eta} & W_{32}^{m \eta} & W_{33}^{m \eta} & 0 & 0 & 0 \\
0 & 0 & 0 & T_{11}^{m \eta} & T_{12}^{m \eta} & 0 \\
0 & 0 & 0 & T_{21}^{m \eta} & T_{22}^{m \eta} & 0 \\
0 & 0 & 0 & T_{31}^{m \eta} & T_{32}^{m \eta} & 0
\end{array}\right] J(\xi) d \xi\left\{\begin{array}{c}
p_{1}^{m \eta} \\
p_{2}^{m \eta} \\
t_{3}^{m \eta} \\
t_{1}^{m \eta} \\
t_{2}^{m \eta} \\
0
\end{array}\right\}
\end{aligned}
$$

Para a qual a composição das funções de forma tem a seguinte configuração:

$$
\boldsymbol{\Phi}=\left[\begin{array}{cccccc}
N_{\eta}^{d} & 0 & 0 & 0 & 0 & 0 \\
0 & N_{\eta}^{d} & 0 & 0 & 0 & 0 \\
0 & 0 & N_{\eta}^{d} & 0 & 0 & 0 \\
0 & 0 & 0 & N_{\eta}^{d} & 0 & 0 \\
0 & 0 & 0 & 0 & N_{\eta}^{d} & 0 \\
0 & 0 & 0 & N_{\eta}^{d} & N_{\eta}^{d} & 0
\end{array}\right]
$$

Nestas equações é possível notar que há um desacoplamento entre as formulações e para uma mesma placa, as respostas devido à extensão e flexão são independentes. 


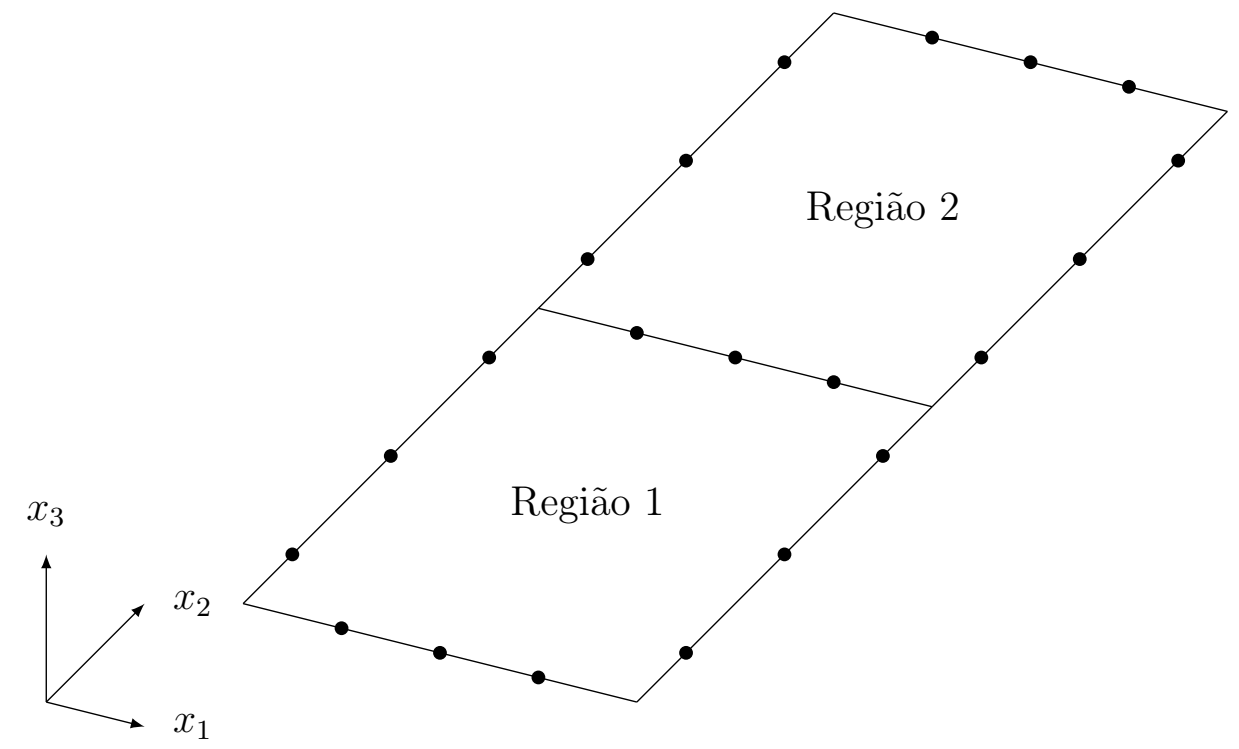

Figura 5.1: Representação esquemática de duas placas coplanares.

Contudo, quando duas placas acopladas por uma aresta estão contidas em diferentes planos, as respostas oriundas da flexão em uma placa podem afetar os graus de liberdade de extensão na outra e vice versa. Isso se deve à imposição de compatibilidades para deslocamentos e forças de superfície generalizados para sistemas de coordenadas diferentes. A próxima seção detalha melhor o motivo desta ocorrência.

\subsection{MEC com sub regiões}

Pelo fato da possibilidade de termos uma ou mais placas unidas por arestas, a abordagem aqui empregada é o método das sub regiões, o que torna necessário a imposição das condições de compatibilidade e equilíbrio ao longo de uma aresta comum.

Em se tratando de um caso simples de placas que pertencem ao mesmo plano, mostrado na Figura 5.1, condições de compatibilidade para deslocamentos $u_{i}$ e rotações $\omega_{i}$ são dadas por:

$$
\begin{aligned}
& u_{i}^{1}=u_{i}^{2} \\
& \omega_{i}^{1}=\omega_{i}^{2}
\end{aligned}
$$

onde o sobrescrito se refere ao número da região. Para o equilíbrio de forças de superfície $t_{i}$ e momentos $p_{i}[24,46]$ : 


$$
\begin{aligned}
& \sum_{m=1}^{M} t_{i}^{m}=0 \\
& \sum_{m=1}^{M} p_{i}^{m}=0
\end{aligned}
$$

onde $M$ é o número de regiões conectadas. Agora, considerando a estrutura representada na Figura 5.2, pode-se analisar um caso mais geral, onde as placas não compartilham o mesmo plano. São dados os sistemas de coordenadas globais $\left(O-x_{1}-x_{2}-x_{3}\right)$ e locais $\left(O-x_{1}^{\prime}-x_{2}^{\prime}-x_{3}^{\prime}\right)$. Com o intuito de impor as condições de compatibilidade e equilíbrio na aresta, agora é necessário transformar as variáveis para o sistema de referência global. Seguindo o apresentado em [3], é necessário utilizar a matriz de transformação, T, o que resulta em:

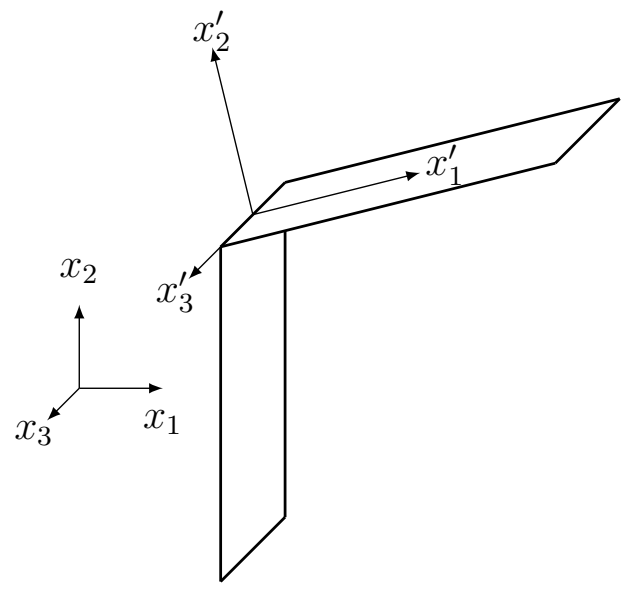

Figura 5.2: Associação de placas mostrando os sistemas de coordenada global e local.

$$
[\mathbf{T}]\left\{\begin{array}{l}
u_{1}^{\prime} \\
u_{2}^{\prime} \\
u_{3}^{\prime}
\end{array}\right\}=\left\{\begin{array}{l}
u_{1} \\
u_{2} \\
u_{3}
\end{array}\right\}, \quad[\mathbf{T}]\left\{\begin{array}{l}
w_{2}^{\prime} \\
w_{1}^{\prime} \\
w_{3}^{\prime}
\end{array}\right\}=\left\{\begin{array}{l}
\theta_{1} \\
\theta_{2} \\
\theta_{3}
\end{array}\right\}
$$

para os deslocamentos generalizados e

$$
[\mathbf{T}]\left\{\begin{array}{c}
t_{1}^{\prime} \\
t_{2}^{\prime} \\
p_{3}^{\prime}
\end{array}\right\}=\left\{\begin{array}{c}
t_{1} \\
t_{2} \\
t_{3}
\end{array}\right\}, \quad[\mathbf{T}]\left\{\begin{array}{c}
-p_{2}^{\prime} \\
p_{1}^{\prime} \\
0
\end{array}\right\}=\left\{\begin{array}{c}
m_{1} \\
m_{2} \\
m_{3}
\end{array}\right\}
$$

para as forças de superfície generalizadas. A matriz $T$ é formada pelos vetores unitários $\left(\mathbf{v}^{1}, \mathbf{v}^{2}, \mathbf{v}^{3}\right)$ nas direções dos eixos coordenados locais $\mathbf{x}_{1}, \mathbf{x}_{2}$ e $\mathbf{x}_{3}$, dada por: 


$$
T=\left[\begin{array}{rrr}
\mathbf{v}_{1}^{1} & \mathbf{v}_{2}^{1} & \mathbf{v}_{3}^{1} \\
\mathbf{v}_{1}^{2} & \mathbf{v}_{2}^{2} & \mathbf{v}_{3}^{2} \\
\mathbf{v}_{1}^{3} & \mathbf{v}_{2}^{3} & \mathbf{v}_{3}^{3}
\end{array}\right]^{-1}
$$

Utilizando procedimento análogo àquele descrito para o caso de duas placas coplanares, agora é possível impor as condições de compatibilidade e equilíbrio para o sistema de equações geral.

Arranjando as equações de diferentes regiões em um único sistema, a forma matricial para as placas associadas fica:

$$
\begin{aligned}
& {\left[\begin{array}{cccccc}
\mathbf{H}_{1}^{(1)} & \mathbf{H}_{12}^{(1)} & \mathbf{0} & \mathbf{0} & -\mathbf{G}_{12}^{(1)} & \mathbf{0} \\
\mathbf{0} & \mathbf{0} & \mathbf{H}_{2}^{(2)} & -\mathbf{H}_{21}^{(2)} & \mathbf{0} & -\mathbf{G}_{21}^{(2)} \\
\mathbf{0} & \mathbf{0} & \mathbf{0} & \mathbf{0} & \mathbf{E}_{12} & \mathbf{E}_{21} \\
\mathbf{0} & \mathbf{C}_{12} & \mathbf{0} & \mathbf{C}_{21} & \mathbf{0} & \mathbf{0}
\end{array}\right]\left\{\begin{array}{c}
u_{1}^{(1)} \\
u_{12}^{(1)} \\
u_{2}^{(2)} \\
u_{21}^{(2)} \\
t_{12}^{(1)} \\
t_{21}^{(2)}
\end{array}\right\}=\left[\begin{array}{cc}
\mathbf{G}_{1}^{(1)} & \mathbf{0} \\
\mathbf{0} & \mathbf{G}_{2}^{(2)}
\end{array}\right]\left\{\begin{array}{c}
t_{1}^{(1)} \\
t_{2}^{(2)}
\end{array}\right\} } \\
&+\left\{\begin{array}{c}
b_{1}^{(1)} \\
b_{12}^{(1)} \\
b_{2}^{(2)} \\
b_{21}^{(2)} \\
0 \\
0
\end{array}\right\}
\end{aligned}
$$

na qual o sobre escrito $(i)$ indica a sub região do problema, os sub escritos 1 e 2 indicam os elementos das matrizes e vetores pertencentes somente àquela região. Os sub escritos cruzados 12 e 21 indicam elementos das matrizes e vetores pertencentes à região 1 e na interface com a 2 e vice versa. As matrizes $\mathbf{E}_{12}$ e $\mathbf{E}_{21}$ são devido às condições de equilíbrio, $\mathbf{C}_{12}$ e $\mathbf{C}_{21}$ são aquelas devido às condições de compatibilidade, dadas por:

$$
E_{12}=\left[\begin{array}{cccccc}
0 & 0 & T_{13}^{(1)} & T_{11}^{(1)} & T_{12}^{(1)} & 0 \\
0 & 0 & T_{33}^{(1)} & T_{31}^{(1)} & T_{32}^{(1)} & 0 \\
0 & 0 & T_{23}^{(1)} & T_{21}^{(1)} & T_{22}^{(1)} & 0 \\
T_{12}^{(1)} & T_{11}^{(1)} & 0 & 0 & 0 & T_{13}^{(1)} \\
T_{22}^{(1)} & T_{21}^{(1)} & 0 & 0 & 0 & T_{23}^{(1)} \\
T_{32}^{(1)} & T_{31}^{(1)} & 0 & 0 & 0 & T_{33}^{(1)}
\end{array}\right]
$$




$$
E_{21}=\left[\begin{array}{cccccc}
0 & 0 & T_{13}^{(2)} & T_{11}^{(2)} & T_{12}^{(2)} & 0 \\
0 & 0 & T_{33}^{(2)} & T_{31}^{(2)} & T_{32}^{(2)} & 0 \\
0 & 0 & T_{23}^{(2)} & T_{21}^{(2)} & T_{22}^{(2)} & 0 \\
T_{12}^{(2)} & T_{11}^{(2)} & 0 & 0 & 0 & T_{13}^{(2)} \\
T_{22}^{(2)} & T_{21}^{(2)} & 0 & 0 & 0 & T_{23}^{(2)} \\
T_{32}^{(2)} & T_{31}^{(2)} & 0 & 0 & 0 & T_{33}^{(2)}
\end{array}\right]
$$

e

$$
\begin{gathered}
C_{12}=\left[\begin{array}{cccccc}
0 & 0 & T_{13}^{(1)} & T_{11}^{(1)} & T_{12}^{(1)} & 0 \\
0 & 0 & T_{33}^{(1)} & T_{31}^{(1)} & T_{32}^{(1)} & 0 \\
0 & 0 & T_{23}^{(1)} & T_{21}^{(1)} & T_{22}^{(1)} & 0 \\
T_{12}^{(1)} & T_{11}^{(1)} & 0 & 0 & 0 & T_{13}^{(1)} \\
T_{22}^{(1)} & T_{21}^{(1)} & 0 & 0 & 0 & T_{23}^{(1)} \\
T_{32}^{(1)} & T_{31}^{(1)} & 0 & 0 & 0 & T_{33}^{(1)}
\end{array}\right] \\
C_{21}=\left[\begin{array}{ccccccc}
0 & 0 & -T_{13}^{(2)} & -T_{11}^{(2)} & -T_{12}^{(2)} & 0 \\
0 & 0 & -T_{33}^{(2)} & -T_{31}^{(2)} & -T_{32}^{(2)} & 0 \\
0 & 0 & -T_{23}^{(2)} & -T_{21}^{(2)} & -T_{22}^{(2)} & 0 \\
-T_{12}^{(2)} & -T_{11}^{(2)} & 0 & 0 & 0 & -T_{13}^{(2)} \\
-T_{22}^{(2)} & -T_{21}^{(2)} & 0 & 0 & 0 & -T_{23}^{(2)} \\
-T_{32}^{(2)} & -T_{31}^{(2)} & 0 & 0 & 0 & -T_{33}^{(2)}
\end{array}\right]
\end{gathered}
$$

\subsection{Aspectos computacionais}

As rotinas usadas para gerar os resultados desta tese, partem de códigos já implementados [47] e foram alterados de maneira a acomodar esta nova versão da formulação para associação de placas deformáveis por cisalhamento e da elasticidade plana com rotação. Todos os programas foram implementadas em código do software Matlab. A maior parte dos procedimentos computacionais já foram descritos nesta tese de maneira implícita.

Os programas desenvolvidos seguem a seguinte rotina básica:

1. Entrada de dados;

2. Geração de visualização da geometria e das condições de contorno do problema (Figura 5.3);

3. Verificação de interfaces e sub regiões;

4. Geração de Malha para o problema; 


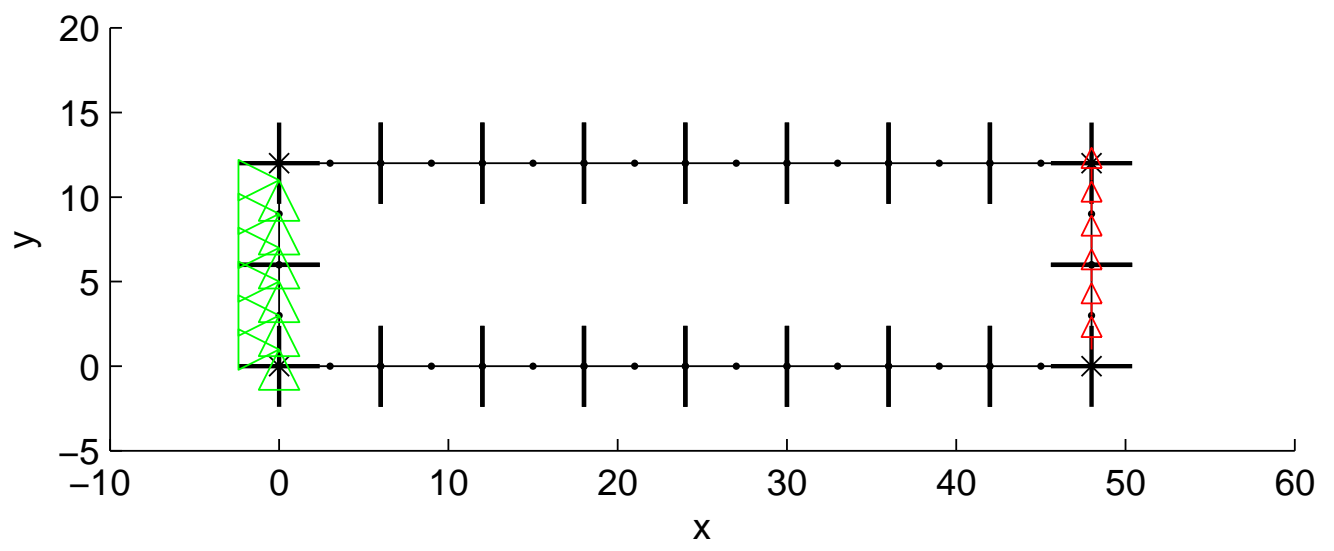

Figura 5.3: Exemplo de visualização de geometria e condições de contorno.

5. Montagem das matrizes de influência para cada uma das sub regiões com resolução das integrais;

6. Posicionamento destas matrizes no sistema global;

7. Imposição de condições de compatibilidade e equilíbrio;

8. Solução do sistema equivalente por eliminação gaussiana;

9. Transformação dos deslocamentos e forças de superfície para o sistema global;

10. Geração de tabelas e gráficos contendo as respostas para visualização e avaliação.

Todas as integrações numéricas realizadas utilizam 10 pontos de Gauss, a menos que seja informado outro valor explicitamente. 


\section{RESULTADOS NUMÉRICOS PARA ELASTICIDADE PLANA}

\subsection{Introdução}

Este capítulo apresenta os resultados obtidos para a rotação $\omega_{3}$ em problemas de elasticidade plana (tensão plana). As respostas serão comparadas com soluções analíticas, sempre que estas estiverem disponíveis, e com os resultados obtidos utilizando a formulação desenvolvida em Leung \& Baiz (2013) [25]. Os erros relativos apresentados neste capítulo e no seguinte foram calculados sempre que havia uma solução analítica disponível e foram obtidos a partir da equação (6.1):

$$
\operatorname{Erro}(\%)=\operatorname{abs}\left(\frac{R^{\text {num }}-R^{\text {an }}}{R^{\text {an }}}\right) \times 100
$$

onde $R^{\text {num }}$ é a resposta numérica e $R^{a n}$ a analítica.

\subsection{Solução analítica bidimensional para uma viga}

Um dos problemas a ser tratado nesta seção é o caso da viga bidimensional. A solução para este problema foi encontrada por Timoshenko [48]. Trata-se da solução bidimensional para uma viga em balanço, submetida a uma carga cisalhante distribuída em uma de suas extremidades. As expressões para os deslocamentos nas direções $x_{1} \mathrm{e}$ $x_{2}$ são apresentados, respectivamente, nas equações (6.2) e (6.3).

$$
\begin{aligned}
& u_{1}\left(x_{1}, x_{2}\right)=-\frac{P x_{2}}{6 E I}\left[(\nu+2)\left(x_{2}^{2}-\frac{h^{2}}{4}\right)+\left(6 L-3 x_{1}\right) x_{1}\right] \\
& u_{2}\left(x_{1}, x_{2}\right)=\frac{P}{6 E I}\left[\frac{h^{2}}{4}(4+5 \nu) x_{1}+3 \nu\left(L-x_{1}\right) x_{2}^{2}+\left(3 L-x_{1}\right) x_{1}^{2}\right]
\end{aligned}
$$

Uma vez que se dispõe de expressões para $u_{1}$ e $u_{2}$, uma expressão analítica para a drilling rotation pode ser obtida a partir de:

$$
w\left(x_{1}, x_{2}\right)=\frac{1}{2}\left(\frac{\partial u_{2}\left(x_{1}, x_{2}\right)}{\partial x_{1}}-\frac{\partial u_{1}\left(x_{1}, x_{2}\right)}{\partial x_{2}}\right)
$$

Substituindo (6.2) e (6.3) em (6.4), tem-se:

$$
w\left(x_{1}, x_{2}\right)=\frac{P}{24 E I}\left[b^{2}(2 \nu+1)+12\left(2 L x_{1}-x_{1}^{2}+x_{2}^{2}\right)\right]
$$

Augarde e Deeks, 2008 [49] analisaram o efeito devido às diferentes maneiras de se impor as condições de contorno para este problema, concluindo que esta solução 
específica não é completamente válida para as condições de contorno comumente utilizadas em simulações numéricas, principalmente próximo à região com deslocamentos restritos. Esta conclusão possui grande importância para a avaliação de esquemas adaptativos de análise, uma vez que a distribuição de tensão é diferente daquela dada pela solução analítica. Apesar disso, de acordo com o princípio de Saint-Venant, esta solução é aplicável para a região de interesse, ou seja, o lado oposto à região engastada da viga. Uma comparação mais precisa pode ser obtida utilizando exatamente as mesmas condições de contorno usadas na obtenção da solução analítica de Timoshenko.

\subsection{Viga em balanço com baixa razão de aspecto}

A análise da viga em balanço mostrada na Figura 6.1 é um dos testes mais comumente utilizados para a avaliação de novos elementos que incluem a drilling rotation. Este problema foi utilizados em vários artigos [25, 31, 34] e foi desenvolvido para avaliar o desempenho de elementos usados para problemas de tensão plana em relação à flexão no plano por pesquisadores do MEF [28]. A razão de aspecto desta viga é $4: 1$, ou seja, 4 unidades de comprimento na horizontal para 1 unidade na vertical. Por conta disso, é referida nesta tese como "viga com baixa razão de aspecto"ou "viga curta".

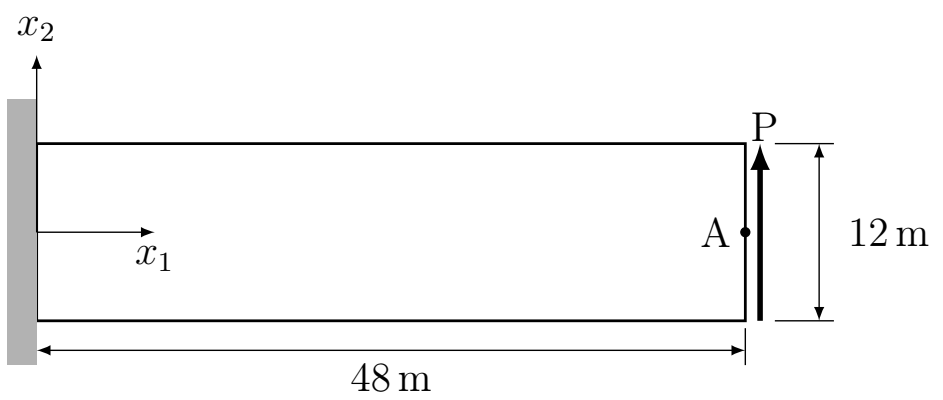

Figura 6.1: Ilustração da viga em balanço. A carga cisalhante distribuída na ponta da viga tem valor resultante $P=40 \mathrm{~N}$, a espessura é $t=1 \mathrm{~m}$.

A solução analítica para este problema é mostrada na seção 6.2 desta tese. Muitos dos trabalhos anteriores usaram a solução analítica de Timoshenko para o deslocamento vertical, direção $x_{2}$, porém nenhum utilizou a solução analítica para a drilling rotation dada pela equação (6.5). Aplicando as equações (6.3) e (6.5) e utilizando as propriedades do problema, $E=30 \mathrm{kPa}, \nu=0.25$, é possível encontrar os seguintes resultados para o ponto $A\left(x_{1}=48, x_{2}=0\right)$ :

$$
\begin{aligned}
& u_{2}=0.355333 \mathrm{~m} \\
& \omega_{3}=0.010750
\end{aligned}
$$


Os resultados analíticos e aqueles obtidos em [25] para elementos lineares totalmente descontínuos são usados para comparação e verificação de convergência. A Figura 6.2 mostra um exemplo de malha com 20 elementos lineares descontínuos.

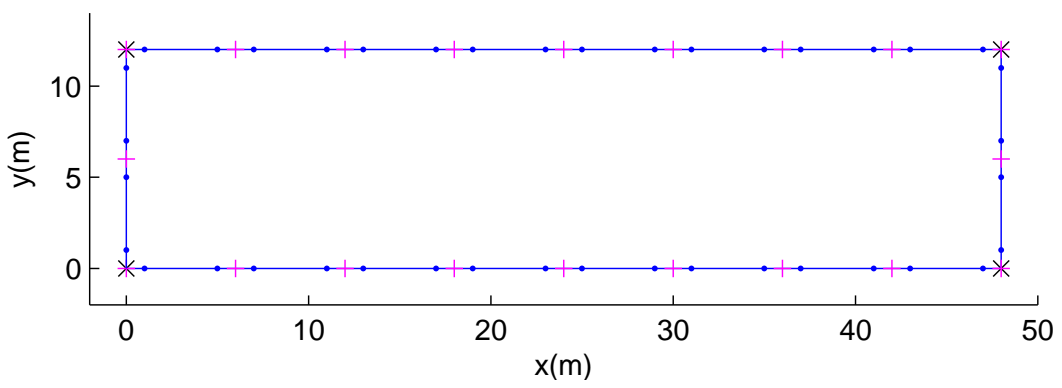

Figura 6.2: Exemplo de um modelo discretizado em elementos de contorno lineares descontínuos para a viga curta. Neste caso foram utilizados 20 elementos.

As Figuras 6.3 e 6.4 apresentam os resultados obtidos em função do número de pontos de colocação, para este caso, dois por elemento. Nesta simulação foram considerados que os nós da aresta na posição $x_{1}=0 \mathrm{~m}$ possuem os deslocamentos restritos nas direções $x_{1}$ e $x_{2}$. O carregamento distribuído é imposto na direção $x_{2}$ e localizado na posição $x_{1}=48 \mathrm{~m}$. É possível verificar que os deslocamentos verticais são quase os mesmos, contudo a rotação $\omega_{3}$ apresenta comportamentos distintos para as duas formulações. Ambos convergem com malhas mais refinadas porém para valores diferentes.

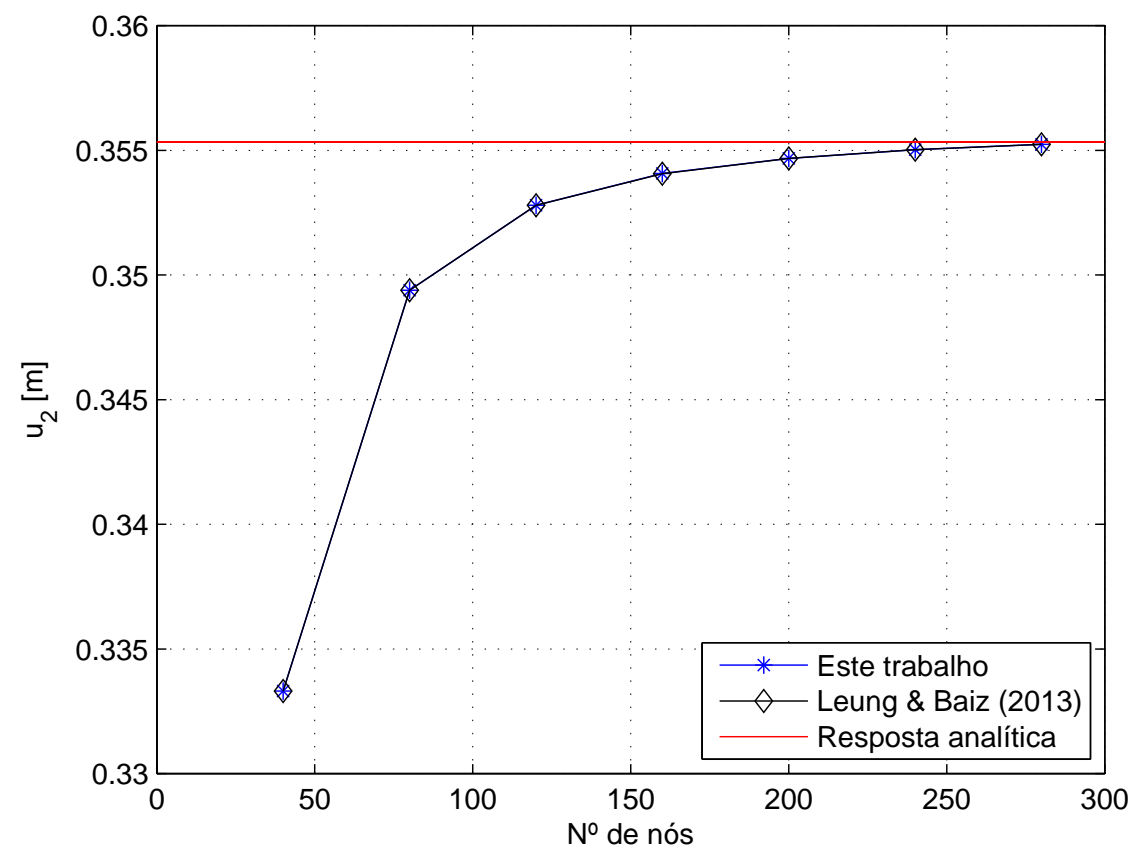

Figura 6.3: Análise de convergência do deslocamento $u_{2}$ no ponto A para a viga curta. 


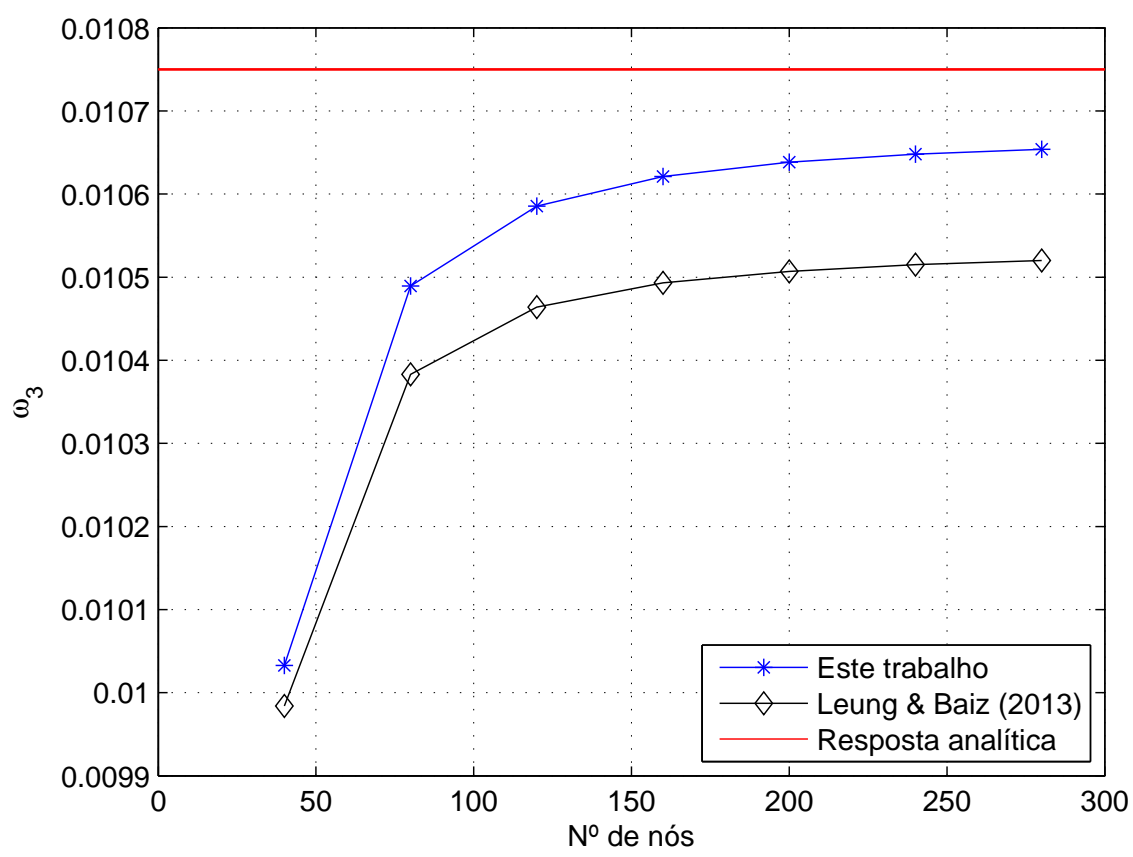

Figura 6.4: Análise de convergência da rotação $\omega_{3}$ no ponto A para a viga curta.

Os erros relativos foram calculados de acordo com a equação (6.1) utilizando como soluções analíticas os valores dados em (6.6). Os resultados são apresentados nas Figuras 6.5 e 6.6 em escala logarítmica.

A Tabela 6.1 mostra a comparação dos resultados para o caso da malha mais refinada usada nesta análise, que contém 280 nós, considerando a formulação proposta e aquela apresentada em [25]. Os resultados foram obtidos usando o mesmo tipo de elemento e as malhas são exatamente iguais. É possível notar que os resultados deste trabalho possuem melhor acordo com as soluções analíticas para a drilling rotation, com resultados cujo erro é inferior a 1\%. A Tabela 6.2 mostra os valores de deslocamento vertical e drilling rotations no ponto $A\left(x_{1}=48, x_{2}=0\right)$ para cada uma das malhas utilizadas.

Tabela 6.1: Comparação dos resultados para o ponto $A\left(x_{1}=48, x_{2}=0\right)$, usando 280 nós.

\begin{tabular}{ccc|cc|c}
\hline & $\begin{array}{c}\text { Este } \\
\text { trabalho }\end{array}$ & $\begin{array}{c}\text { Erro } \\
(\%)\end{array}$ & $\begin{array}{c}\text { Leung \& Baiz (2013) } \\
{[25]}\end{array}$ & $\begin{array}{c}\text { Erro } \\
(\%)\end{array}$ & $\begin{array}{c}\text { Analítico } \\
{[48]}\end{array}$ \\
\hline$u_{2}$ & 0.355259 & 0.0264 & 0.355241 & 0.0260 & 0.355333 \\
$\omega$ & 0.010654 & 0.8941 & 0.010520 & 2.1396 & 0.010750 \\
\hline
\end{tabular}




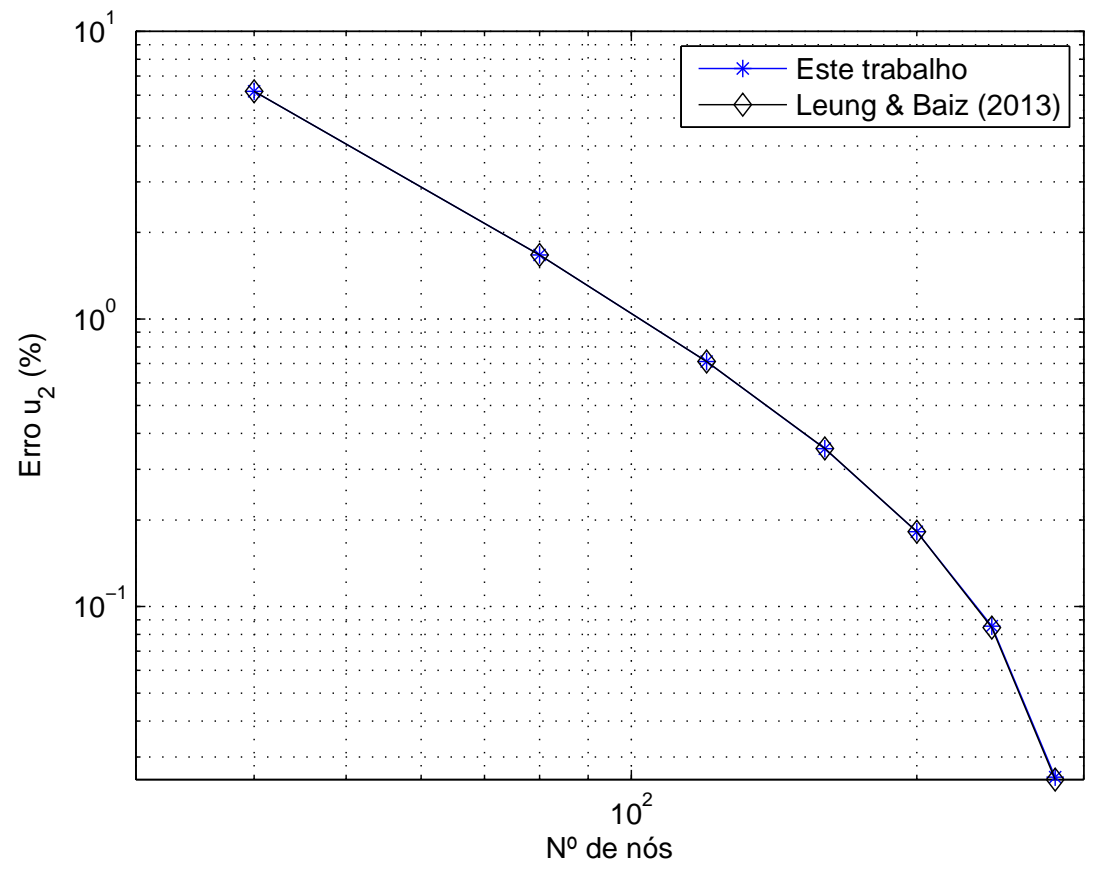

Figura 6.5: Erro calculado para $u_{2}$ em função do número de nós para viga curta.

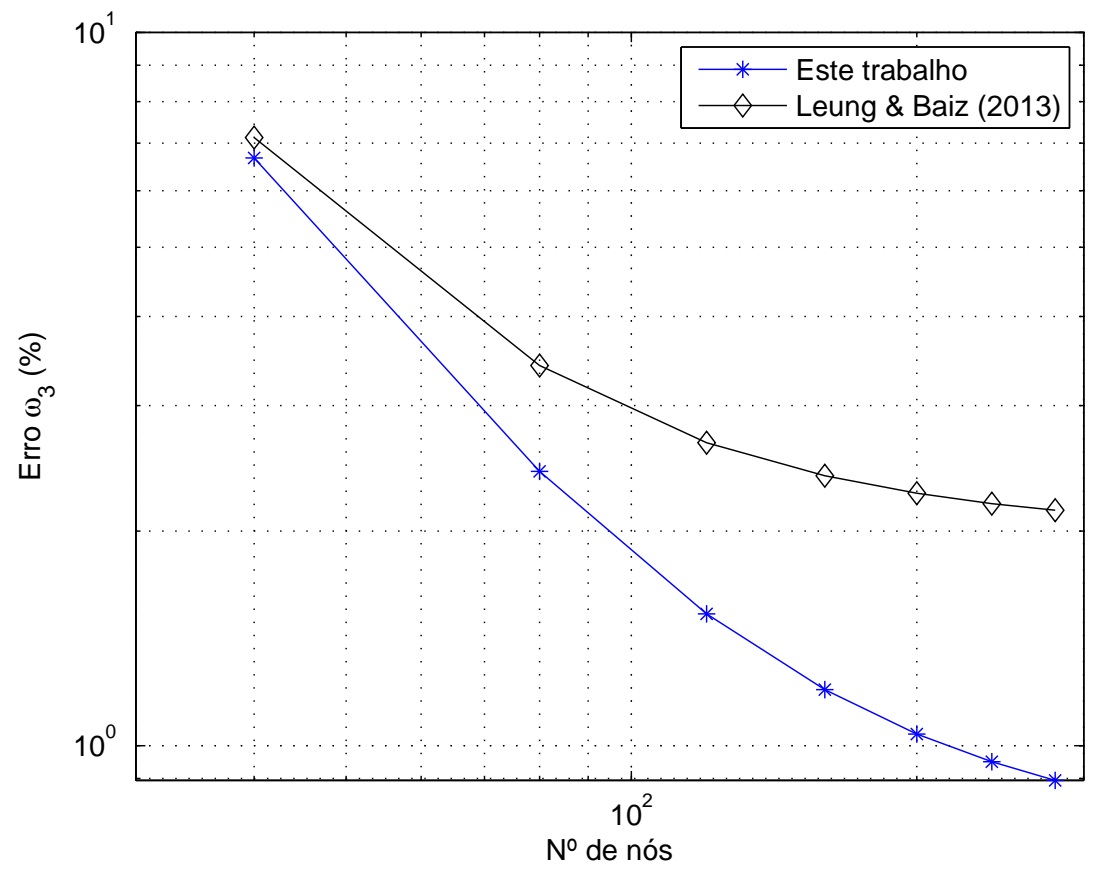

Figura 6.6: Erro calculado para $\omega_{3}$ em função do número de nós para viga curta. 
Tabela 6.2: Resultados obtidos para a viga curta.

\begin{tabular}{ccccc}
\hline $\begin{array}{c}\text { Pontos de } \\
\text { Colocação }\end{array}$ & $\begin{array}{c}u_{2} \\
{[\mathrm{~m}]}\end{array}$ & $\begin{array}{c}u_{2} \text { erro } \\
(\%)\end{array}$ & $\begin{array}{c}\omega_{3} \\
{[]}\end{array}$ & $\begin{array}{c}\omega_{3} \text { erro } \\
(\%)\end{array}$ \\
\hline 40 & 0.333320 & 6.195 & 0.010033 & 6.672 \\
80 & 0.349391 & 1.672 & 0.010489 & 2.425 \\
120 & 0.352802 & 0.712 & 0.010585 & 1.531 \\
160 & 0.354071 & 0.355 & 0.010621 & 1.199 \\
200 & 0.354683 & 0.183 & 0.010638 & 1.039 \\
240 & 0.355027 & 0.086 & 0.010648 & 0.950 \\
280 & 0.355239 & 0.026 & 0.010654 & 0.894 \\
\hline
\end{tabular}

\subsubsection{Resultados para condições de contorno corrigidas}

Como mencionado anteriormente, a solução analítica utilizada foi obtida para um conjunto específico de condições de contorno [48]. Contudo, de acordo com [49] reproduzir exatamente as restrições de deslocamento e carregamentos impostos é essencial para obter resultados comparáveis. A Figura 6.7 mostra as condições de contorno usadas por Timoshenko [48] quando da obtenção desta solução. Uma análise deste problema de acordo com essas condições também foi realizada e os resultados são apresentados a seguir e comparados com a solução analítica.

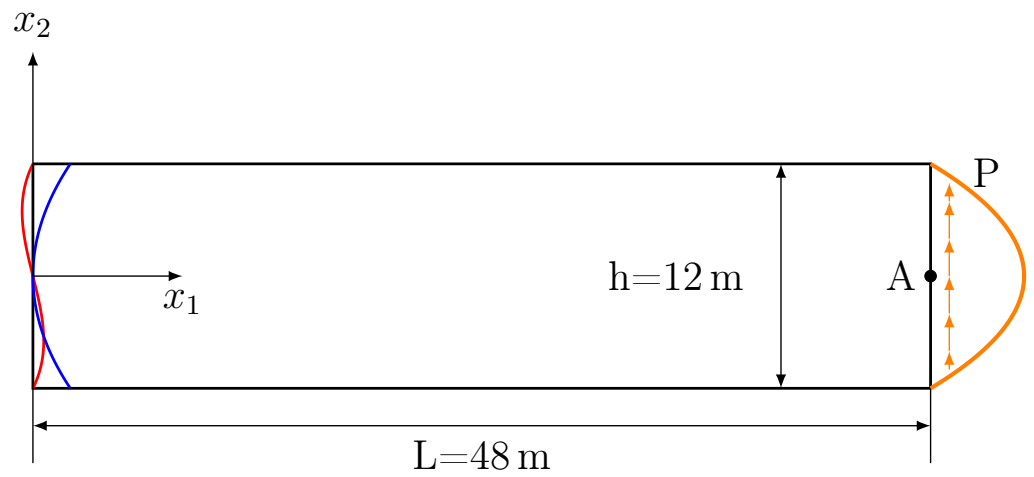

Figura 6.7: Modelo da viga com condições de contorno corrigidas. A resultante de carga na extremidade da viga é igual $P=40 N$ e possui distribuição parabólica. Os deslocamentos $u_{1}$ (vermelho) e $u_{2}$ (azul) variam de acordo com a solução analítica. 


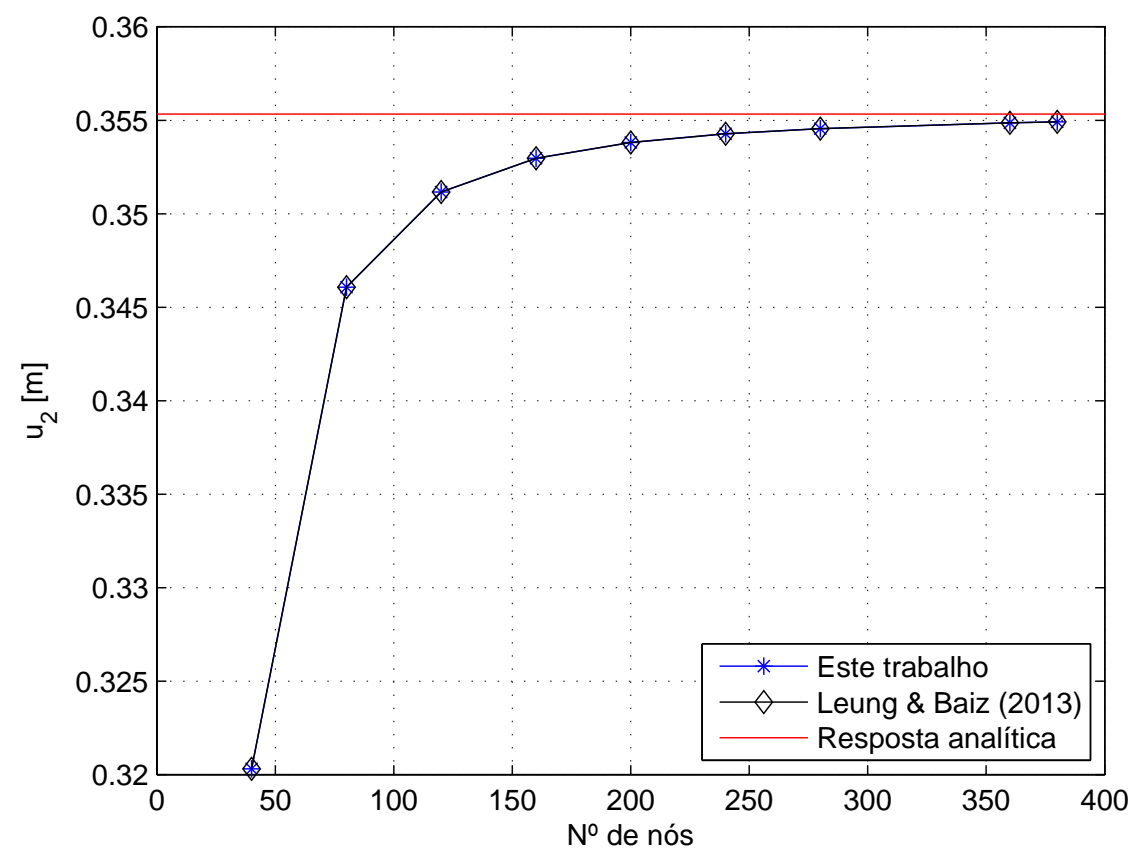

Figura 6.8: Resultados de $u_{2}$ no ponto A para a viga curta com condições de contorno corrigidas.

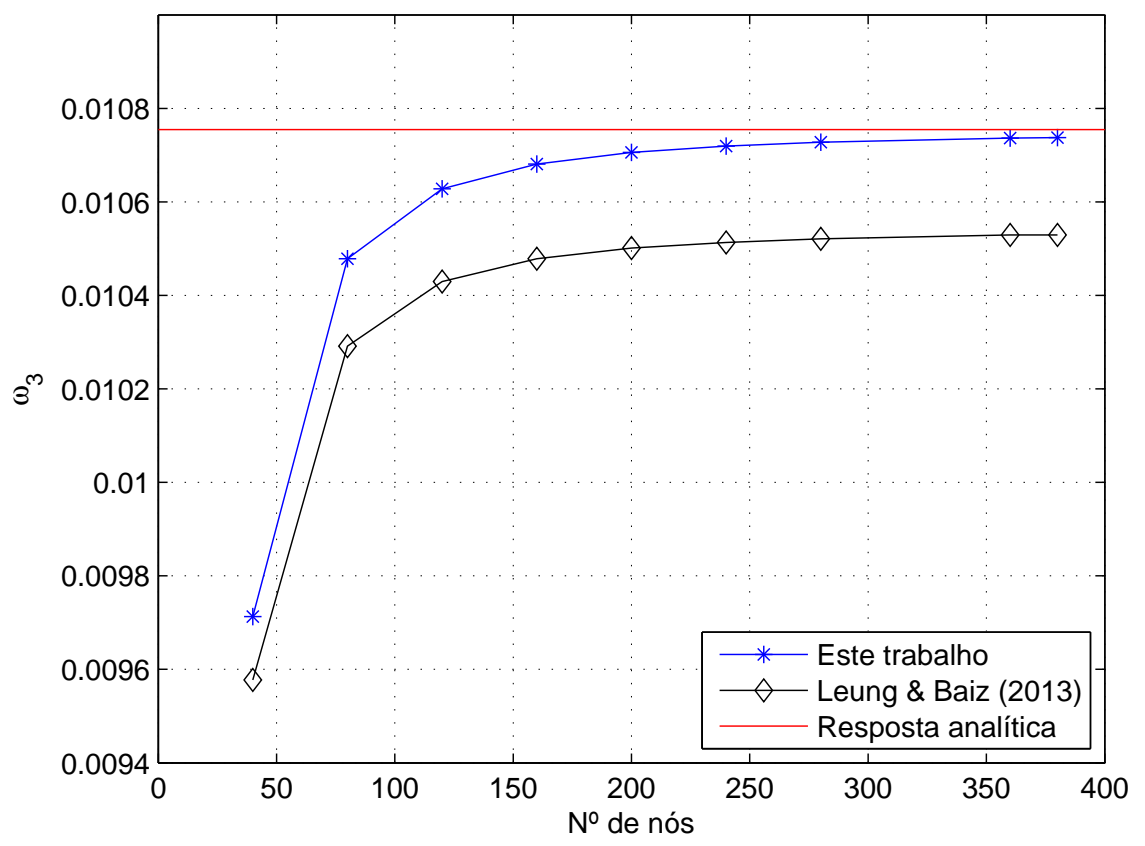

Figura 6.9: Resultados de $\omega_{3}$ no ponto A para a viga curta com condições de contorno corrigidas. 


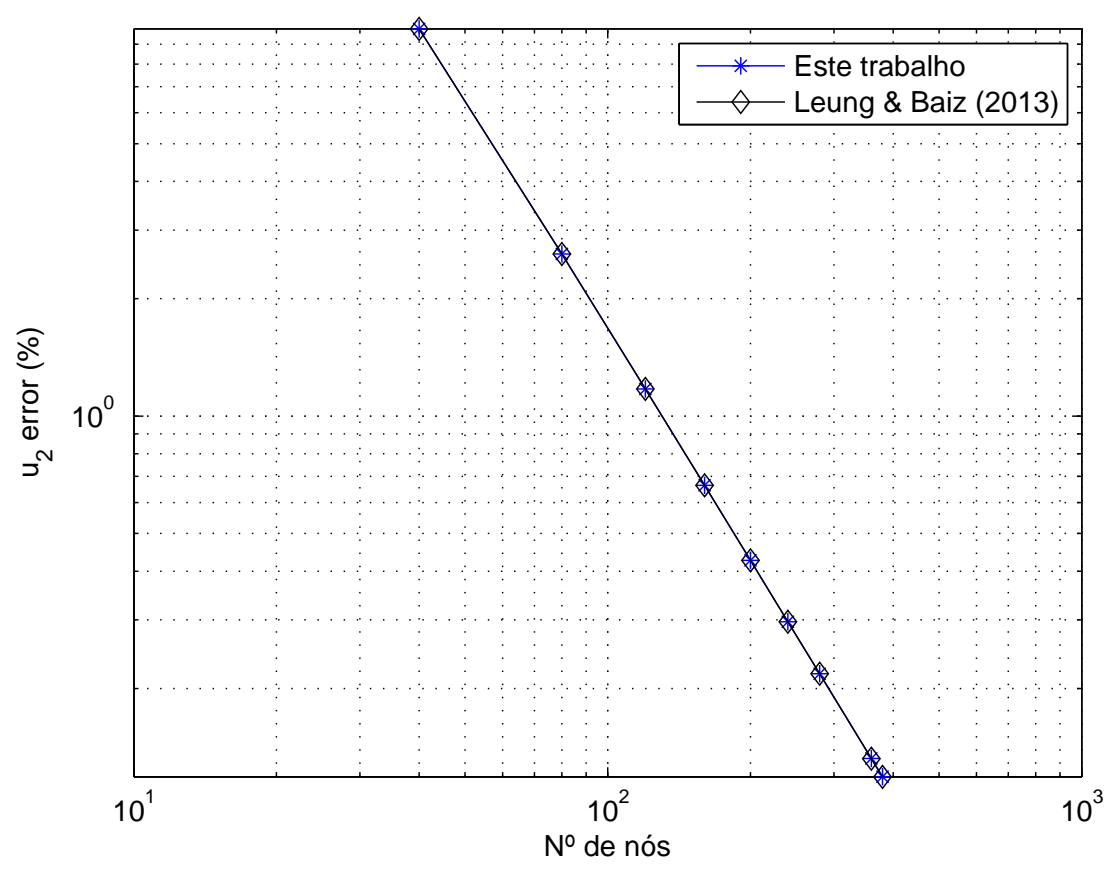

Figura 6.10: Erros relativos para $u_{2}$ em função do número de pontos de colocação em escala logarítmica com condições de contorno corrigidas.

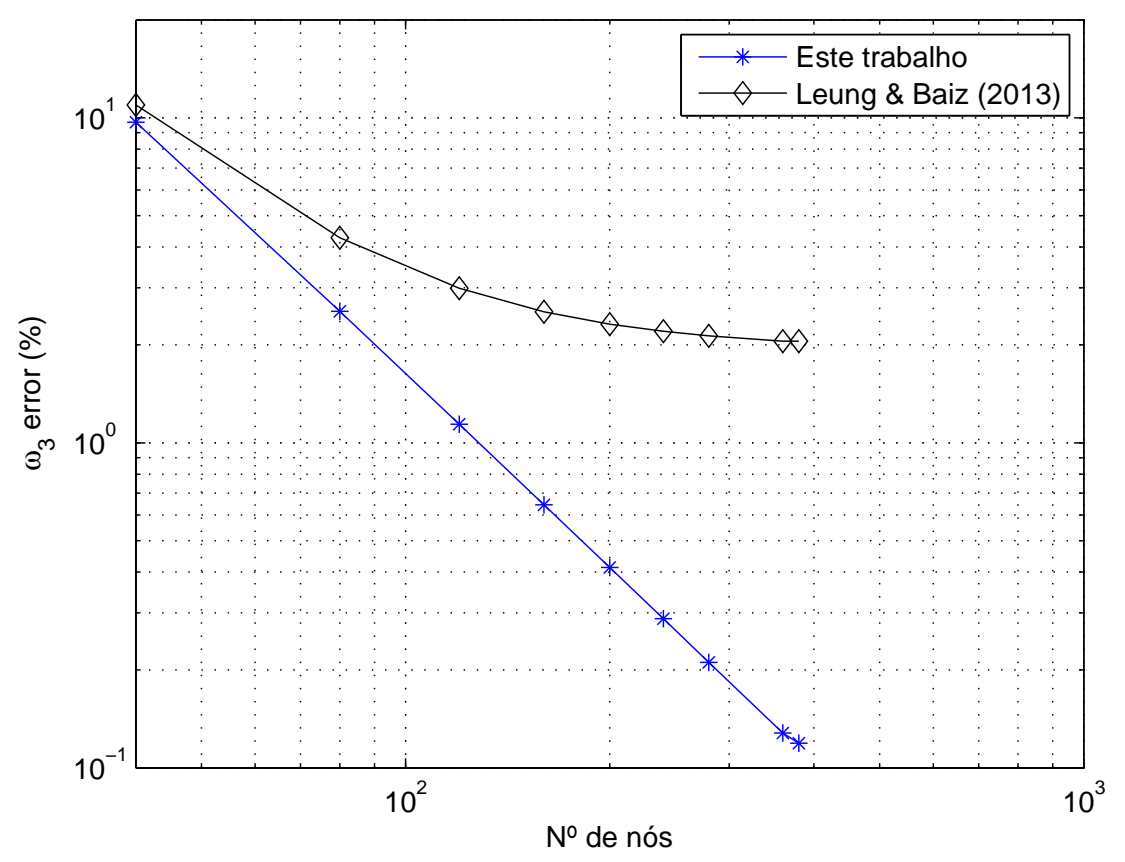

Figura 6.11: Erros relativos para $\omega_{3}$ em função do número de pontos de colocação em escala logarítmica com condições de contorno corrigidas.

Como é possível verificar, os resultados se aproximam ainda mais da solução analítica nas Figuras 6.8 e 6.9 quando comparados com aqueles das Figuras 6.3 e 6.4, especialmente para $\omega_{3}$, indicando que os valores obtidos em trabalhos anteriores se- 
riam melhores se as exatas condições de contorno tivessem sido utilizadas. A Figura 6.11 mostra claramente que a formulação apresentada em [25] não converge para a resposta analítica, enquanto a apresentada neste trabalho converge progressivamente para este valor.

\subsection{Membrana de Cook}

A membrana de Cook é um problema teste proposto para avaliar o caso geral de elementos finitos quadrilaterais em malhas distorcidas. Não possui uma solução analítica conhecida mas vários trabalhos obtiveram soluções numéricas empregando o MEF. Com o intuito de comparar não apenas os deslocamentos verticais mas também a rotação $\omega_{3}$, os resultados em [31] e [25] serão usados. A Figura 6.12 mostra a geometria do problema e suas condições de contorno. Foi utilizado o mesmo número de elementos para cada lado do problema.

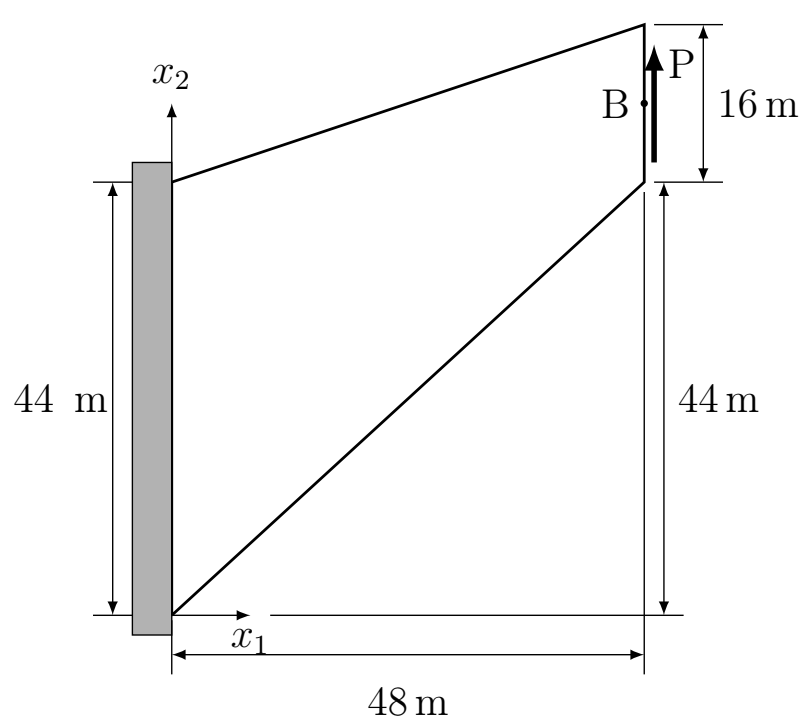

Figura 6.12: Modelo da membrana de Cook. As propriedades mecânicas são: $E=1 \mathrm{~Pa}$, $\nu=1 / 3$. O carregamento distribuído tem resultante $P=1 \mathrm{~N}$ e espessura $t=1 \mathrm{~m}$.

Um exemplo de malha para este problema é mostrado na Figura 6.13. A distribuição de deslocamentos totais para esta geometria é apresentada na Figura 6.14. Os resultados para a convergência desta simulação são apresentados nas Figuras 6.15 e 6.16 para o ponto $\mathrm{B}$, nas quais é possível comparar os desempenhos da formulação proposta e [25]. Assim como no problema anterior, os deslocamentos verticais $u_{2}$ são quase os mesmos enquanto a rotação $\omega_{3}$ converge para valores diferentes. 


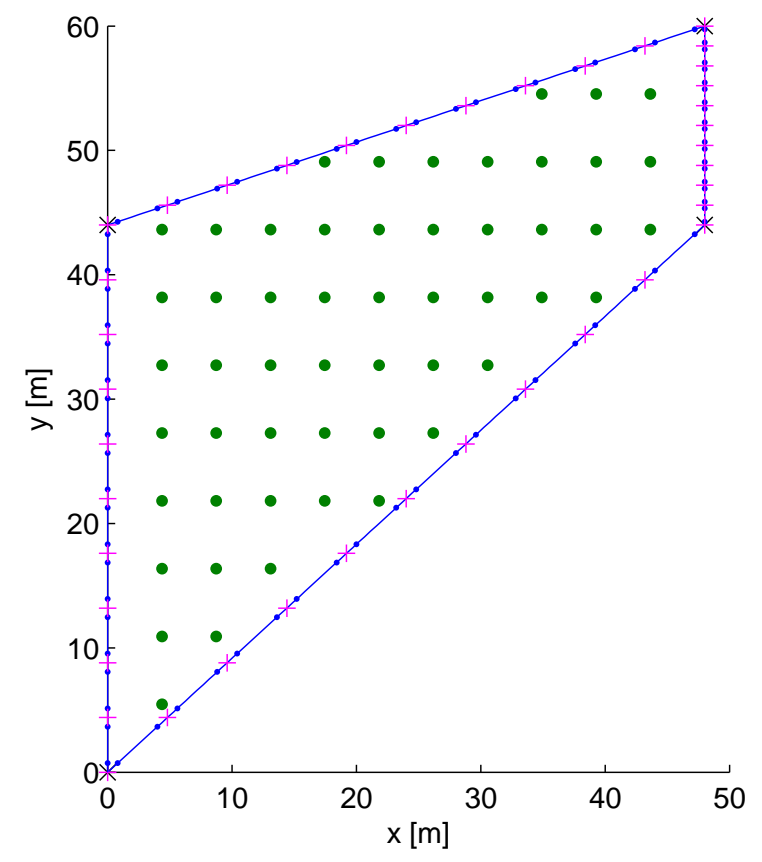

Figura 6.13: Exemplo de malha para a membrana de Cook com 40 elementos lineares descontínuos.

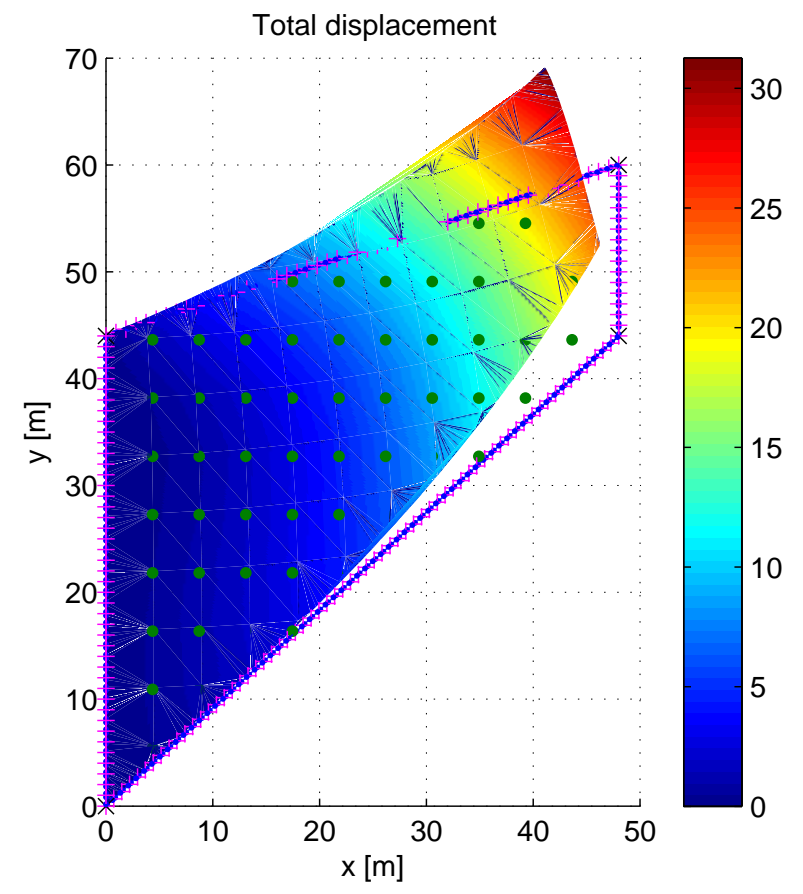

Figura 6.14: Distribuição de deslocamentos totais para a membrana de Cook. 


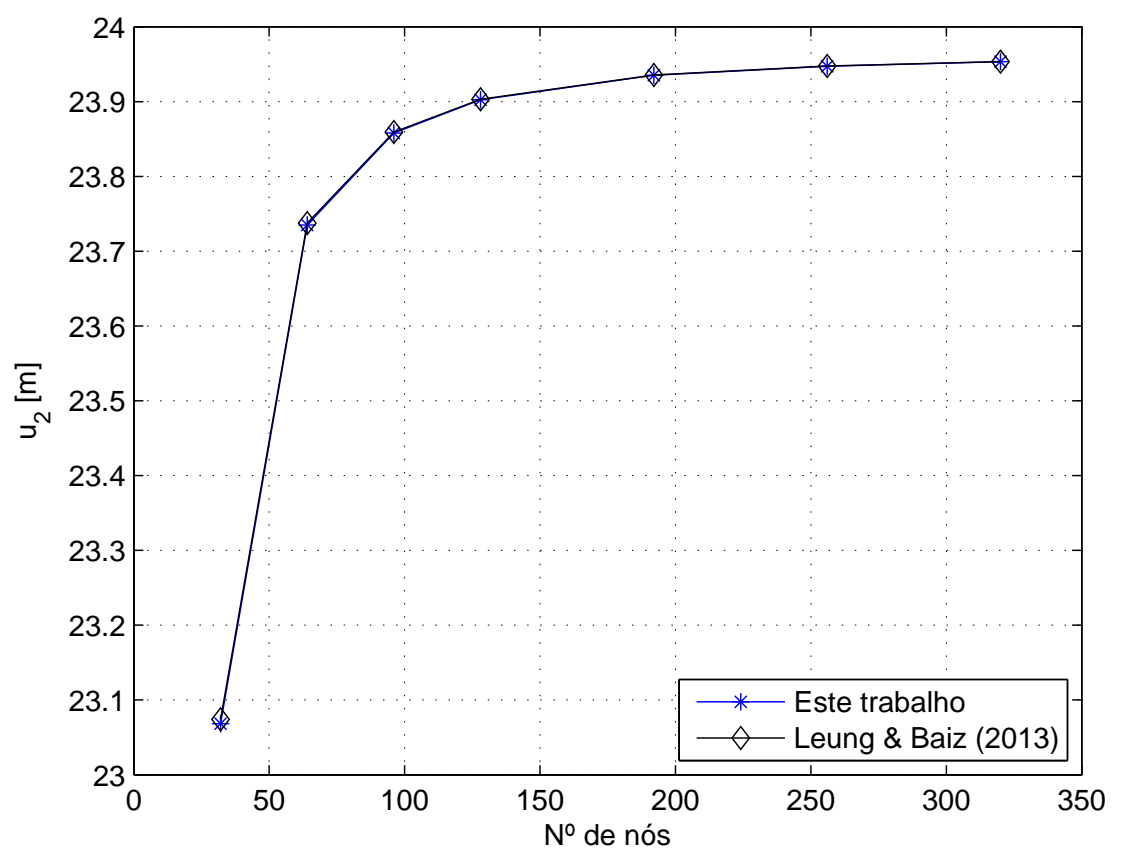

Figura 6.15: Resultados de deslocamentos para a membrana de Cook no ponto $B\left(x_{1}=\right.$ $\left.48, x_{2}=52\right)$.

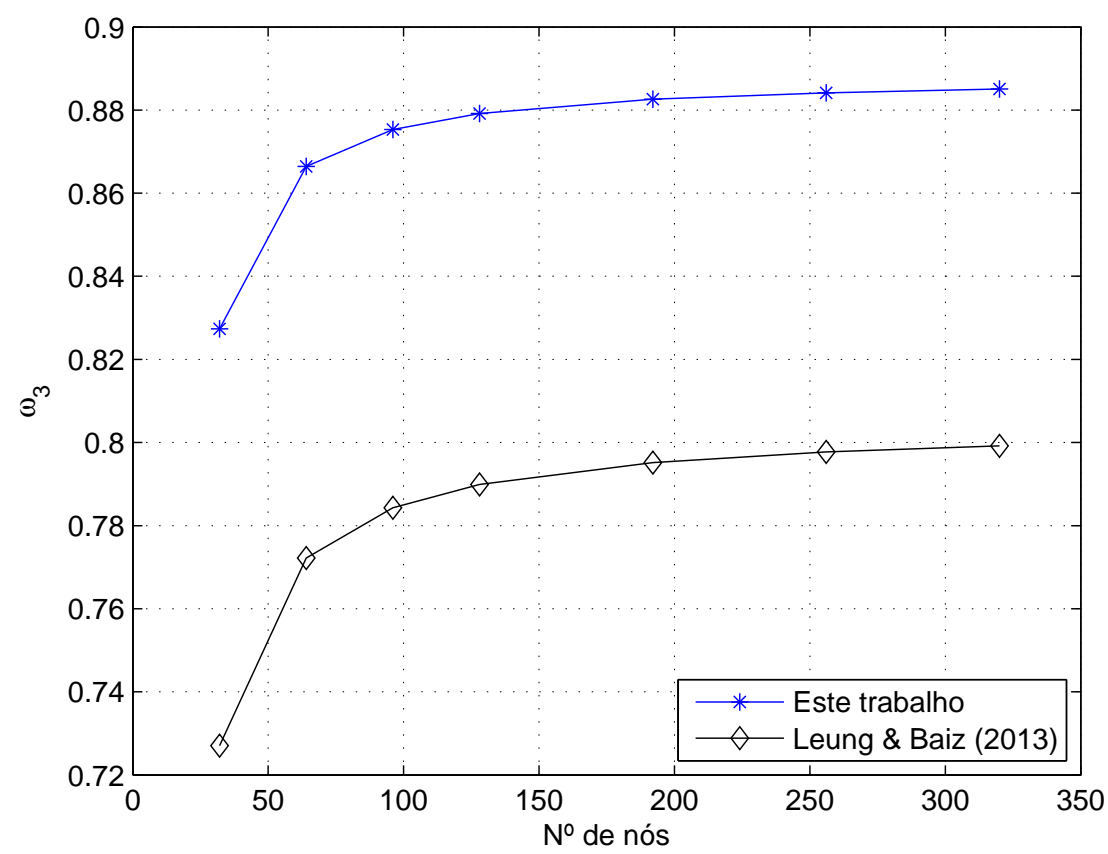

Figura 6.16: Resultados de rotações para a membrana de Cook no ponto $B\left(x_{1}=48, x_{2}=52\right)$.

Por não haver uma solução analítica para este problema, não é possível afirmar qual das soluções possui maior precisão. Entretanto, considerando a Tabela 6.3 que mostra os resultados obtidos em [31] com elementos finitos quadrilaterais (enhanced Allman 
quadrilateral elements) e malhas refinadas, é possível verificar um melhor acordo com os resultados obtidos com a formulação proposta nesta tese.

Tabela 6.3: Resultados para a membrana de Cook usando 320 pontos de colocação. Deslocamentos verticais e drilling rotations no ponto $B\left(x_{1}=48, x_{2}=52\right)$.

\begin{tabular}{cccc}
\hline & Presente trabalho & {$[25]$} & {$[31]$} \\
\hline$u_{2}$ & 23.95424 & 23.95350 & 23.889 \\
$w_{3}$ & 0.88508 & 0.79919 & 0.88733 \\
\hline
\end{tabular}

A Tabela 6.4 mostra os valores para o deslocamento vertical e a rotação no ponto $B\left(x_{1}=48, x_{2}=52\right)$ para cada malha utilizada.

Tabela 6.4: Resultados para a membrana de Cook.

\begin{tabular}{ccc}
\hline Pontos de colocação & $u_{2}[\mathrm{~m}]$ & $\omega_{3}$ \\
\hline 32 & 23.06819 & 0.82733 \\
64 & 23.73513 & 0.86641 \\
96 & 23.85813 & 0.87532 \\
128 & 23.90233 & 0.87919 \\
192 & 23.93515 & 0.88262 \\
256 & 23.94733 & 0.88416 \\
320 & 23.95329 & 0.88505 \\
\hline
\end{tabular}

\subsection{Viga em balanço com alta razão de aspecto}

Esta seção trata da análise de uma viga, assim como o primeiro exemplo deste capítulo. As considerações feitas em relação às condições de contorno impostas quando da obtenção da solução de Timoshenko, obviamente, também são válidas aqui. Contudo, as dimensões e razão de aspecto da viga, fazem com que a distinção entre as soluções obtidas seja mínima. Por conta disso, as condições de contorno mais simplificadas apresentadas na Figura 6.17 serão utilizadas. Este problema foi resolvido em [31] utilizando o MEF com um elemento que inclui a rotação $\omega_{3}$. A razão de aspecto desta viga é 100 : 1, por conta disso, é referida nesta tese como "viga com alta razão de aspecto"ou "viga fina". A solução analítica é a mesma já apresentada para o primeiro exemplo deste capítulo. Aplicando as equações (6.3) e (6.5) para extremidade carregada da viga, tem-se: 


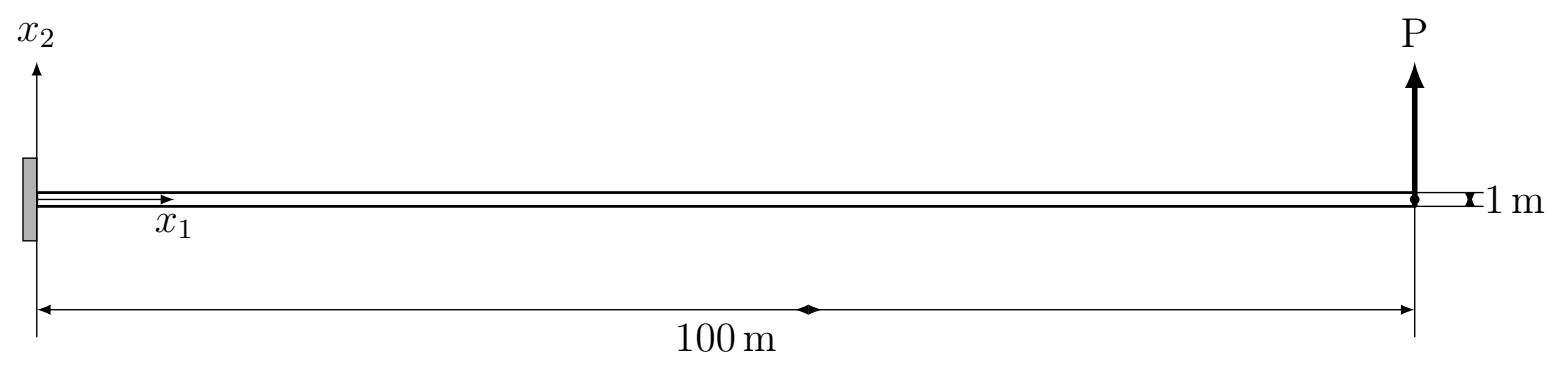

Figura 6.17: Modelo para a viga com alta razão de aspecto. As propriedades mecânicas são $E=1 \mathrm{MPa}, \nu=0.3$. A resultante de carregamento na ponta da viga tem valor $P=1 \mathrm{~N}$ e a espessura é $t=1 \mathrm{~m}$.

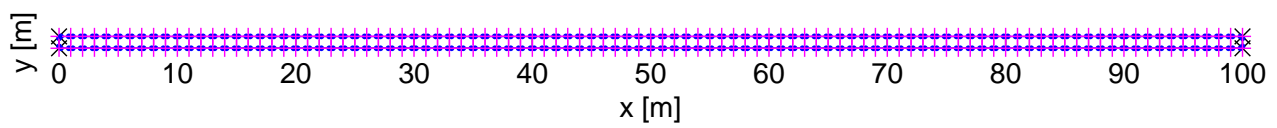

Figura 6.18: Exemplo de malha para a viga com alta razão de aspecto contendo 202 elementos de contorno.

$$
\begin{aligned}
& u_{2}=4.0002 \mathrm{~m} \\
& \omega_{3}=0.0600
\end{aligned}
$$

Um exemplo de malha para este problema é mostrado na Figura 6.18. Os resultados obtidos utilizando-se elementos lineares descontínuos são apresentados nas Figuras $6.19,6.20$. As Tabelas 6.5 e 6.6 apresentam os valores obtidos para cada uma das malhas utilizadas. Nestas tabelas o erros na comparação das soluções analíticas com as respostas numéricas são também apresentados. Para malhas pouco refinadas o erro é inaceitável, atingindo valores de até 82\%. Na malha mais refinada, com 4040 nós, o erro obtido para os deslocamentos foi da ordem de 5.6\%. É possível observar que a convergência é bastante lenta para os deslocamentos verticais e rotações. A partir de 3232 nós, a convergência da rotação para de ocorrer e oscilações do resultado em torno da resposta analítica são observados. Esse comportamento se torna evidente quando observados os erros para as últimas 3 malhas. 


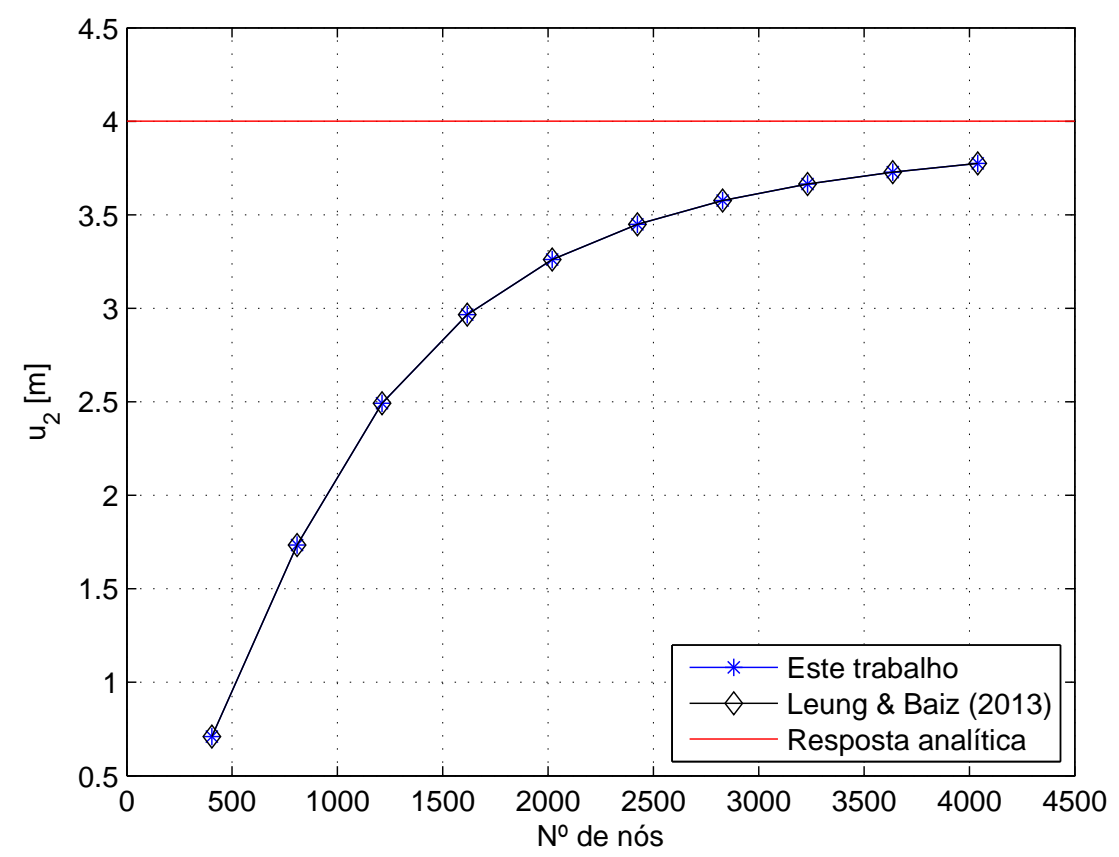

Figura 6.19: Resultados de deslocamentos $u_{2}$ para a viga com alta razão de aspecto na extremidade livre.

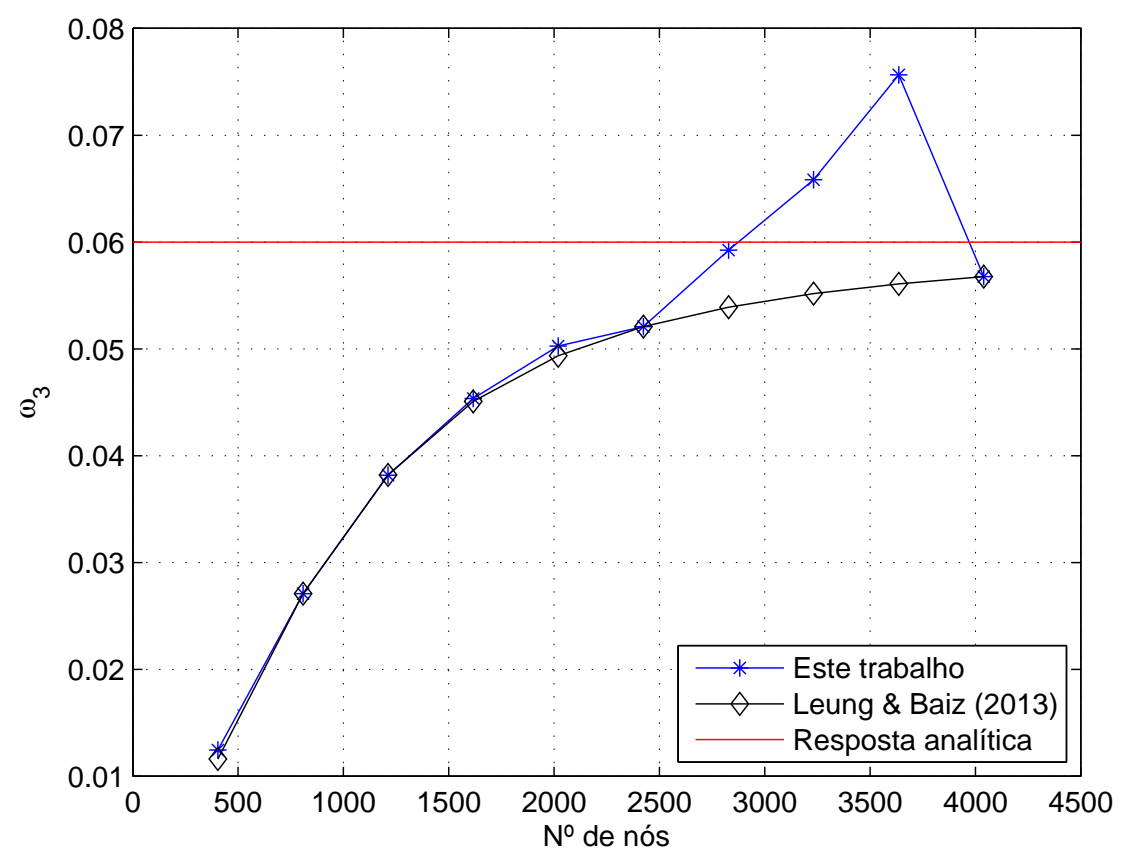

Figura 6.20: Resultados de rotações $\omega_{3}$ para a viga com alta razão de aspecto na extremidade livre.

Este problema de acordo com [38], apresenta sérios problemas para a análise com o MEC devido a relativa proximidade entre os lados de sua geometria, uma vez que sua razão de aspecto é muito alta. 
Tabela 6.5: Resultados para a viga com alta razão de aspecto em $x_{1}=100 \mathrm{~m}$.

\begin{tabular}{ccccc}
\hline Pontos de Colocação & $u_{2}^{a n}=4.000275$ & Erro $(\%)$ & $\omega_{3}^{a n}=0.060001$ & Erro $(\%)$ \\
\hline 404 & 0.709318 & 82.268 & 0.012469 & 79.219 \\
808 & 1.733632 & 56.662 & 0.027092 & 54.847 \\
1212 & 2.491654 & 37.713 & 0.038179 & 36.369 \\
1616 & 2.966058 & 25.854 & 0.045368 & 24.387 \\
2020 & 3.260558 & 18.492 & 0.050264 & 16.228 \\
2424 & 3.449674 & 13.764 & 0.052089 & 13.186 \\
2828 & 3.576264 & 10.600 & 0.059251 & 1.250 \\
3232 & 3.664382 & 8.397 & 0.065844 & -9.739 \\
3636 & 3.727867 & 6.810 & 0.075641 & -26.067 \\
4040 & 3.774979 & 5.632 & 0.056773 & 5.379 \\
\hline
\end{tabular}

Tabela 6.6: Resultados para a viga com alta razão de aspecto. Deslocamentos verticais e rotações na extremidade livre.

\begin{tabular}{cccc}
\hline & Este trabalho & Leung \& Baiz (2013) $[25]$ & Resposta analítica \\
\hline$u_{2}$ & 3.774979 & 3.774979 & 4.000275 \\
$\omega_{3}$ & 0.052089 & 0.056772 & 0.060001 \\
\hline
\end{tabular}

Em [50] foram investigadas as causas da lenta convergência para a solução analítica de um problema semelhante, com razões de aspecto $L / H=5,10,20$, para elementos de contorno constantes. Esta causa foi descartada pois a mesma geometria, sob um carregamento de tração, convergiu rapidamente. Contudo, a proximidade dos elementos pode ser importante para a rotação, que sofreu problemas de convergência assim que o número de pontos de colocação se tornou muito alto. O mesmo problema não foi verificado para a formulação apresentada em [25], o que corrobora a explicação uma vez que o enriquecimento das funções de forma não inclui hiper singularidades no problema. O baixo grau da ordem de interpolação do elemento e sua dificuldade em descrever a forma fletida da viga também é indicado como um fator importante. Neste trabalho foram adotadas duas estratégias diferentes: aplicação de sub regiões, o que torna as distâncias relativas entre os nós maiores e o aumento no número de pontos de Gauss, que melhora a precisão da integração. Os resultados obtidos utilizando a ordem quadrática de interpolação dos elementos serão apresentados paralelamente àqueles obtidos com a formulação linear na próxima seção. 


\subsubsection{Viga com alta razão de aspecto com sub regiões}

A aplicação desta técnica melhora o resultado para este problema por conta da diminuição da razão de aspecto promovida pela divisão do domínio. As comparações serão realizadas dividindo o domínio em 2 e 4 sub regiões. A convergência dos resultados para 2 sub regiões são apresentados nas Figuras 6.21 e 6.22. Para a convergência do problema resolvido com 4 sub regiões as respostas são apresentadas nas Figuras 6.23 e 6.24 .

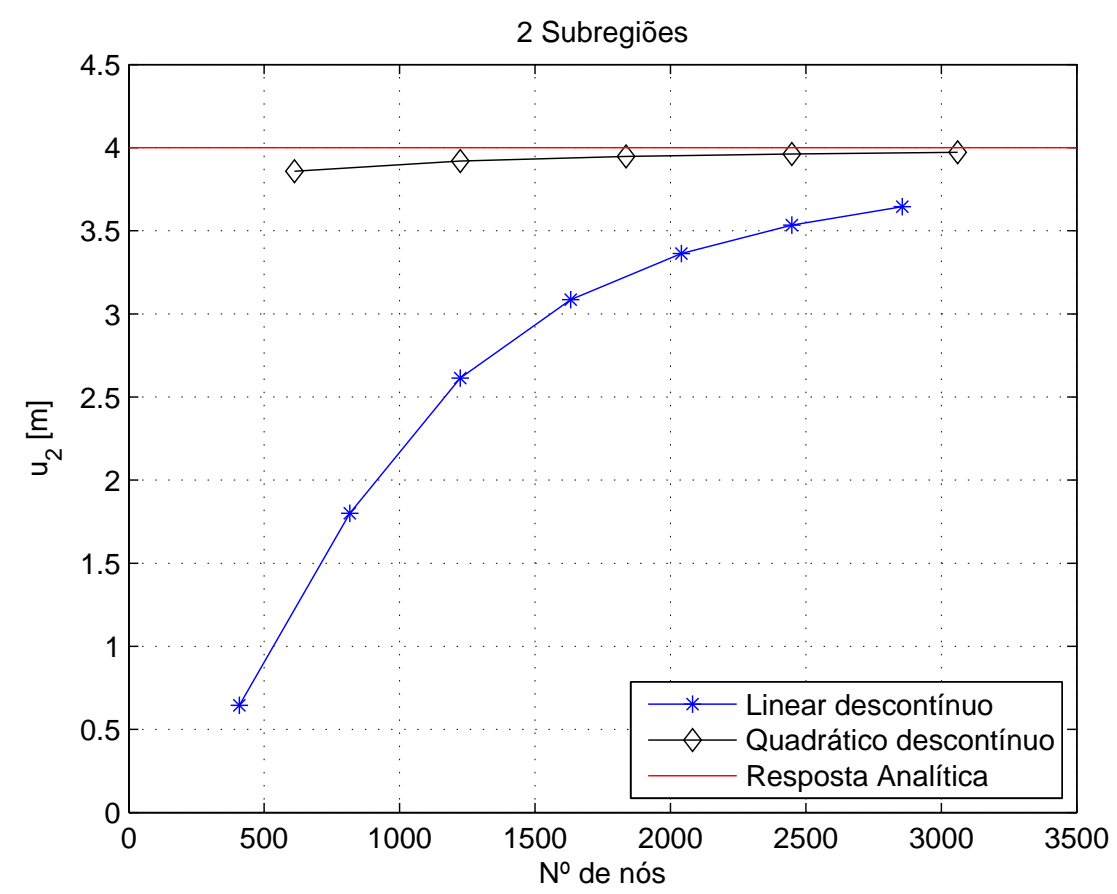

Figura 6.21: Convergência para o deslocamento $u_{2}$ para o caso da viga com alta razão de aspecto e 2 sub regiões. 


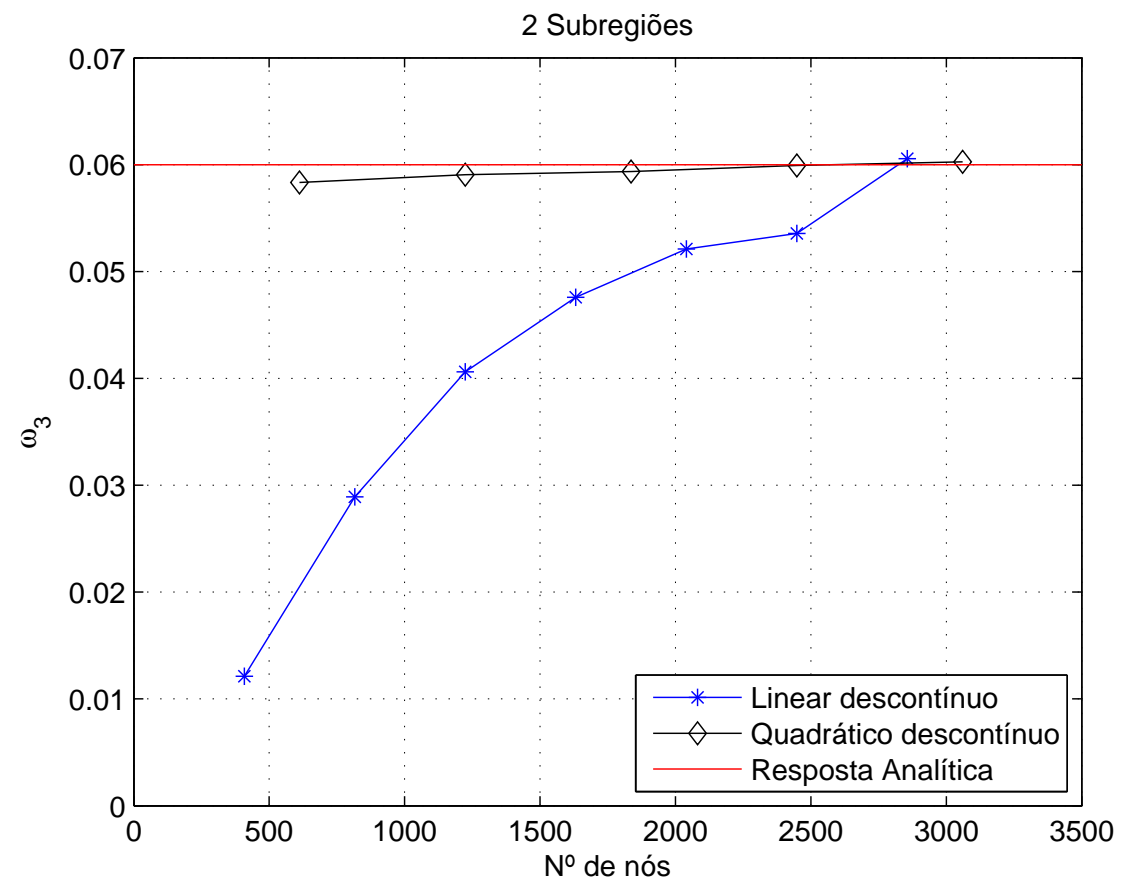

Figura 6.22: Convergência para a rotação $\omega_{3}$ para o caso da viga com alta razão de aspecto e 2 sub regiões.

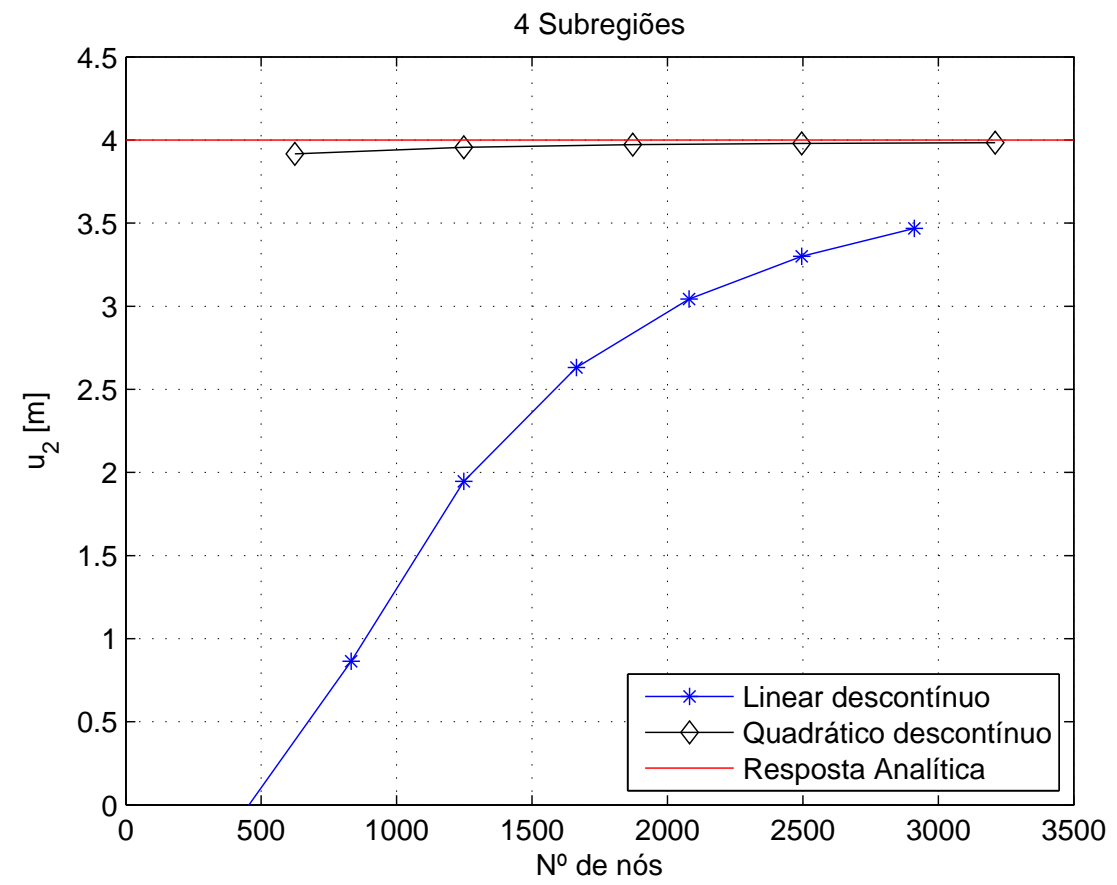

Figura 6.23: Convergência para o deslocamento $u_{2}$ para o caso da viga com alta razão de aspecto e 4 sub regiões. 


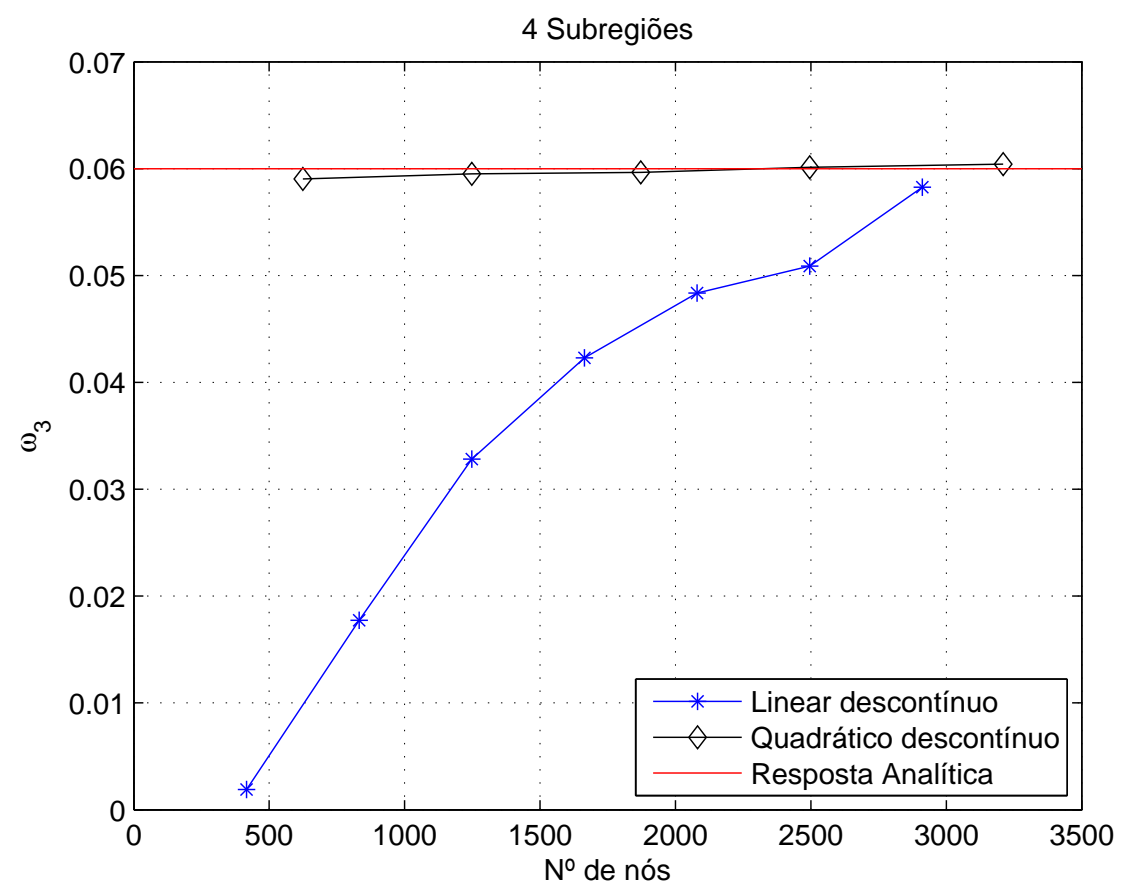

Figura 6.24: Convergência para a rotação $\omega_{3}$ para o caso da viga com alta razão de aspecto e 4 sub regiões.

É possível observar que para elementos quadráticos a convergência se dá de maneira rápida, enquanto para os elementos lineares a discretização mesmo com 4 sub regiões se mostrou pouco efetiva em acelerá-la. A oscilação da resposta para a rotação $\omega_{3}$ é bastante minimizada com elementos quadráticos mas continua ocorrendo com o uso da interpolação linear.

\subsubsection{Viga com alta razão de aspecto com aumento no número de pontos de integração}

Nesta seção os números de pontos de integração serão alterados e o resultados de convergência comparados. As Figuras 6.25 e 6.26 mostram a variação da resposta para diferentes números de pontos de integração $(\mathrm{PG})$ :

Para este caso não foi observada uma variação significativa dos resultados. Portanto a quantidade de pontos de integração tem pouca efetividade na melhoria da convergência.

O aumento no grau da função de interpolação foi a técnica mais efetiva na melhoria da convergência deste problema. Dessa maneira, pode se inferir que o problema está na dificuldade da função linear em reproduzir a forma fletida da viga. Mesma conclusão obtida em [50]. 


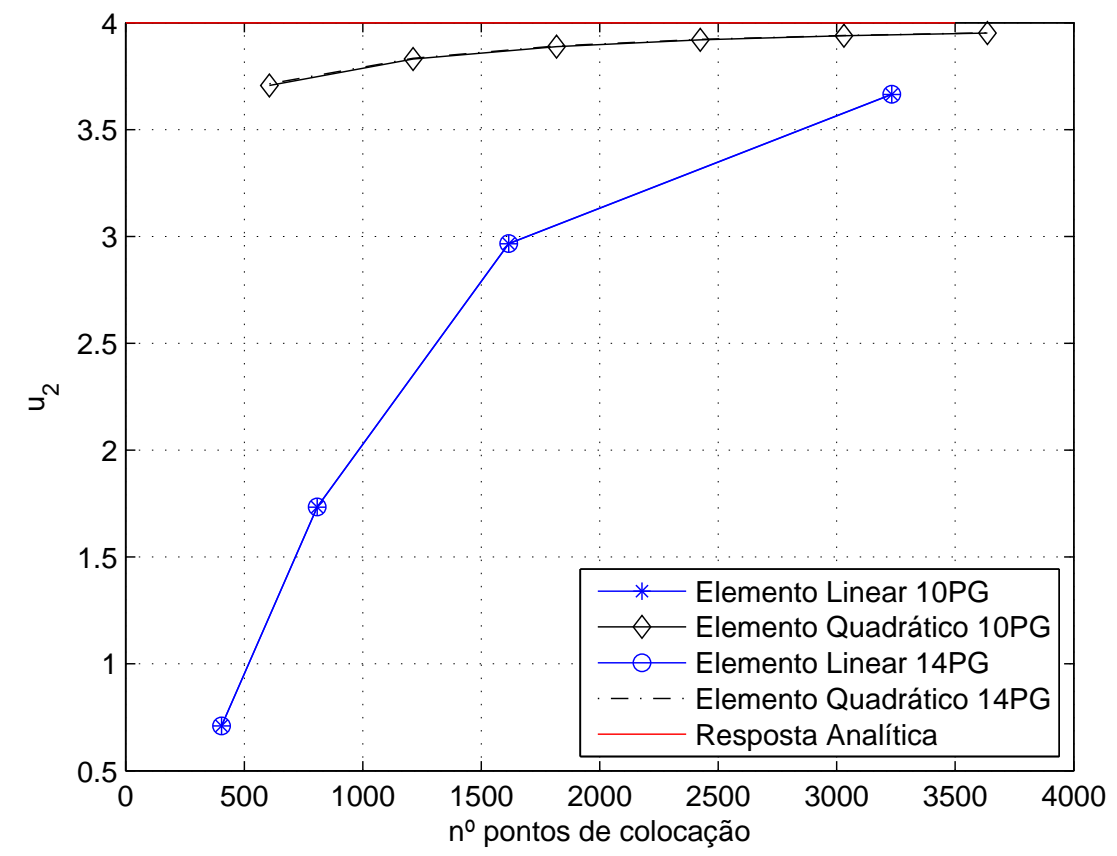

Figura 6.25: Análise de convergência dos deslocamentos $u_{2}$ para a viga com alta razão de aspecto considerando diferentes números de pontos de integração.

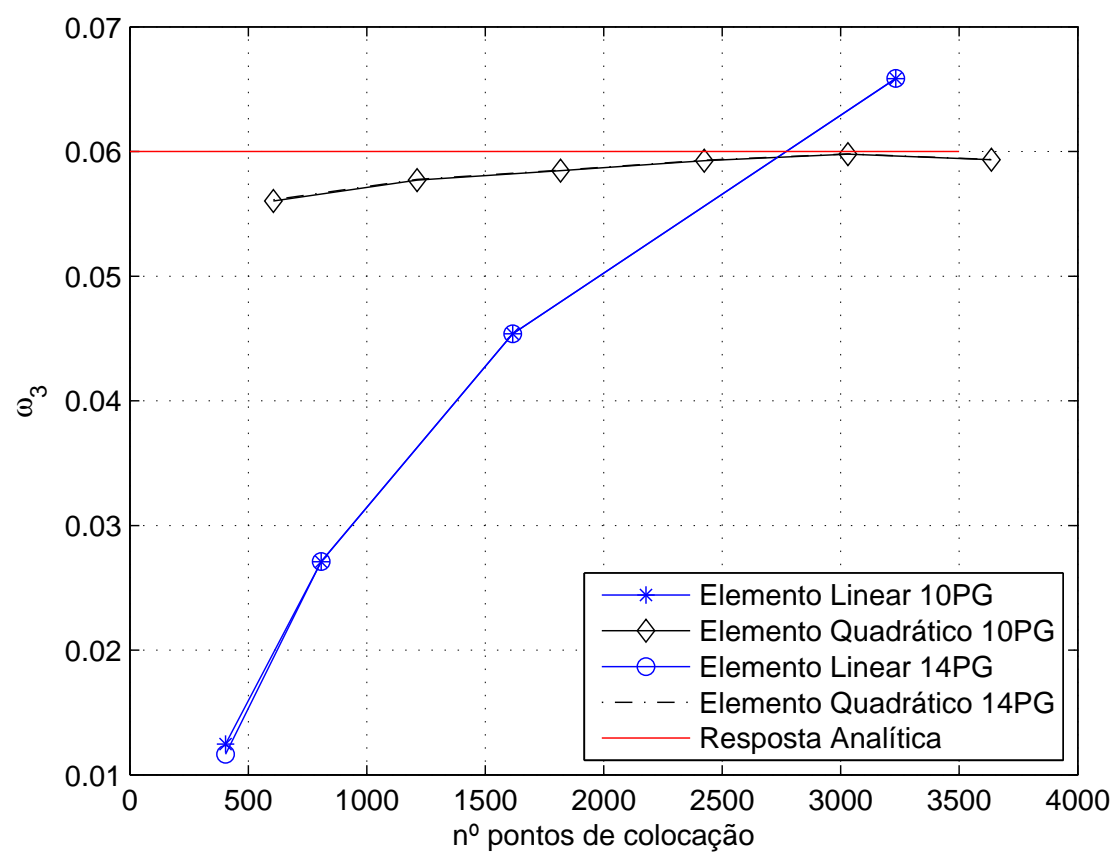

Figura 6.26: Análise de convergência das rotações $\omega_{3}$ para a viga com alta razão de aspecto considerando diferentes números de pontos de integração. 


\section{RESULTADOS NUMÉRICOS PARA ASSOCIAÇÃO DE PLACAS}

\subsection{Introdução}

Os resultados desta seção foram obtidos a partir de um código de elementos de contorno para placas associadas que combina a formulação de elasticidade plana com drilling rotation e a formulação de placas espessas. Os problemas apresentados aqui foram, em sua maioria, analisados anteriormente em [3]. Para validação dos resultados, as respostas desta referência, na qual estão contidas soluções analíticas de alguns problemas, foram utilizadas. Para aqueles problemas que não possuem solução analítica disponível, uma resposta numérica obtida com o MEF com o auxílio do Ansys foi obtida. A análise foi feita utilizando o elemento SHELL181 que é adequado para a análise de estruturas formadas por cascas ou placas de finas a moderadamente espessas. Este elemento é quadrilateral de 4 nós e possui 24 graus de liberdade, 6 para cada um dos nós. Três deslocamentos nas direções $x_{1}, x_{2}$ e $x_{3}$ e três rotações em torno dos eixos $x_{1}, x_{2}$ e $x_{3}[51,52]$.

Os erros relativos apresentados nesta seção foram calculados sempre que havia uma solução analítica disponível e foram obtidos, assim como no capítulo anterior, a partir da equação (6.1).

\subsection{Associação em L}

Este modelo é composto por duas placas associadas de maneira a formar uma estrutura em "L". Este problema foi resolvido em [3], no qual pode ser encontrada a sua solução analítica. O modelo foi discretizado usando duas sub regiões, uma para cada placa, com 12 elementos lineares descontínuos cada. As propriedades e dimensões são dadas na Figura 7.1, onde $L_{1}=1 \mathrm{~m}, L_{2}=1 \mathrm{~m}, L_{3}=2 \mathrm{~m}, t_{1}=0.1 \mathrm{~m}$ e $t_{2}=0.1 \mathrm{~m}$. A carga é distribuída ao longo da aresta superior, orientada na direção $x_{3}$ com valor $q=1 \mathrm{~N} / \mathrm{m}$. As propriedades mecânicas são $E=100 \mathrm{kPa}$ and $\nu=0.0$.

A distribuição do deslocamento total $\left(u_{\text {total }}=\sqrt{u_{1}^{2}+u_{2}^{2}+u_{3}^{2}}\right)$ pode ser visualizada nas Figuras 7.2 e 7.3 para as formulações deste trabalho e o MEC convencional. A Tabela 7.1 apresenta a comparação dos resultados com os obtidos analiticamente em [3] para um ponto na extremidade da placa superior, na mesma aresta que recebe o carregamento. Quando comparado com a solução de Euler-Bernoulli, o erro é de $0.625 \%$ e quando comparado com a solução de Timoshenko é de $0.3741 \%$. Não há diferença significativa entre os resultados das Figuras 7.2 e 7.3 o que é esperado pois 


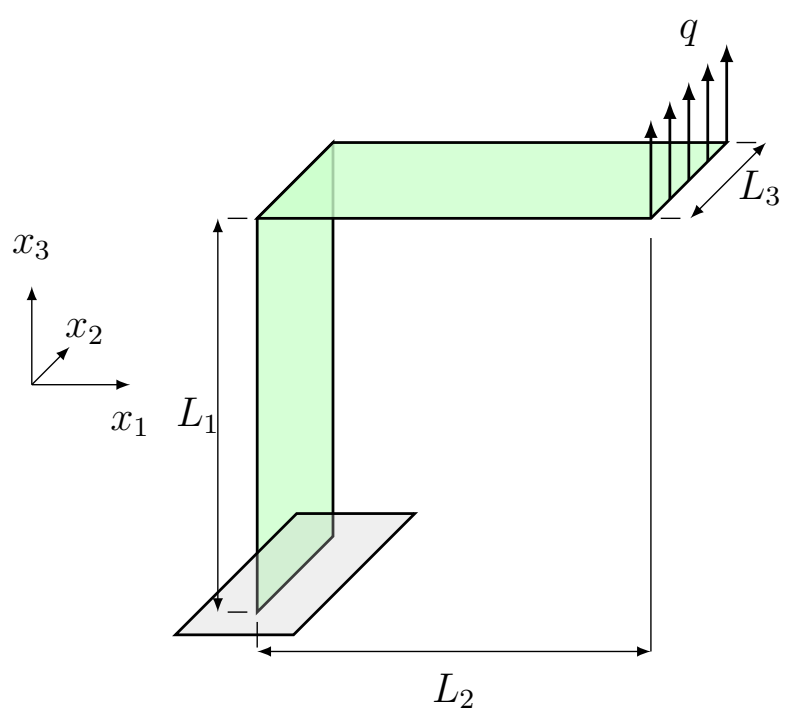

Figura 7.1: Dimensões e condições de contorno para a estrutura em "L" [3].

não são geradas as rotações $\omega_{3}$ no problema.

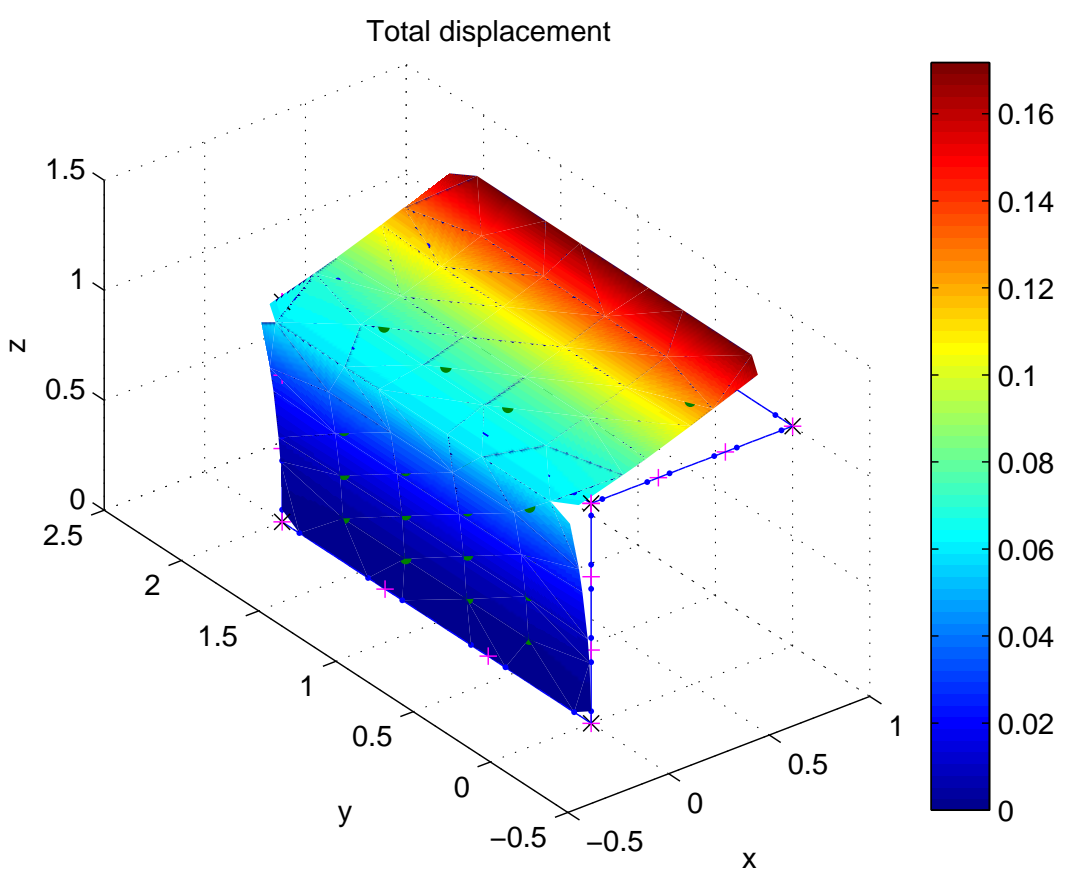

Figura 7.2: Distribuição do deslocamento total para a estrutura em "L" obtidos com a formulação deste trabalho. 


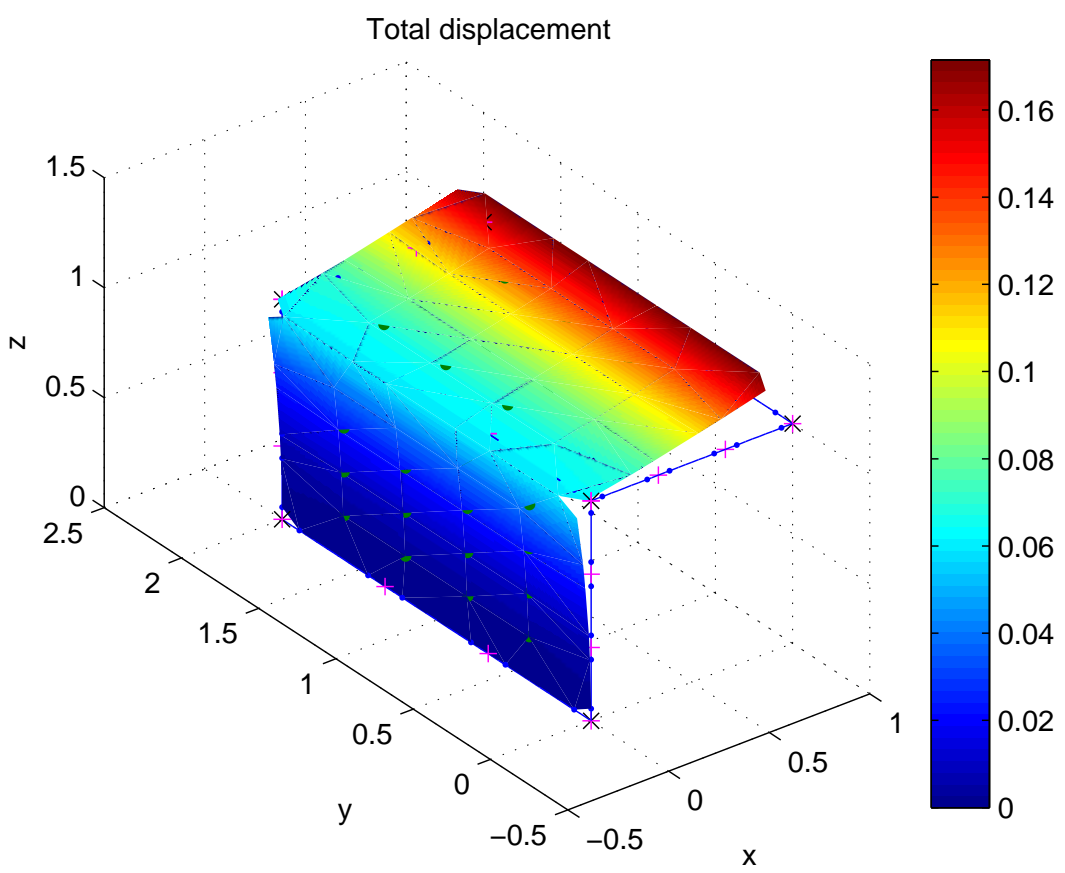

Figura 7.3: Distribuição do deslocamento total para a estrutura em "L" obtidos com a formulação convencional do MEC.

Tabela 7.1: Deslocamento na direção $x_{3}$ calculado na ponta da placa comparado às soluções analíticas.

\begin{tabular}{cc}
\hline Método de Solução & Deslocamento máximo na direção $x_{3}$ \\
\hline MEC convencional & 0.16100 \\
MEC com drilling rotation & 0.16100 \\
Solução de Bernouli Euler & 0.16000 \\
Solução de Timoshenko & 0.16040 \\
\hline
\end{tabular}

\subsection{Associação em L em ângulos não-retos}

Este exemplo é uma variação do problema anterior, com a diferença que o ângulo de junção entre as placas pode ter valores superiores a $90^{\circ}$. Estes exemplos são utilizados para demonstrar a capacidade da formulação em modelar estruturas de placas associadas por um ângulo arbitrário. O modelo foi discretizado usando duas sub regiões, com 12 elementos lineares descontínuos cada. As propriedades e dimensões são mostradas na Figura 7.4, na qual $L_{1}=1 \mathrm{~m}, L_{2}=1 \mathrm{~m}, L_{3}=2 \mathrm{~m}, t_{1}=0.1 \mathrm{~m}$ e $t_{2}=0.1 \mathrm{~m}$. A carga distribuída na borda superior tem valor $q=1 \mathrm{~N} / \mathrm{m}$. As propriedades mecânicas são $E=100 \mathrm{kPa}$ e $\nu=0.0$.

As Figuras de 7.5 a 7.10 apresentam a distribuição de deslocamento total para a estrutura com as duas placas unidas por ângulos de $91^{\circ}, 95^{\circ} \mathrm{e} 120^{\circ}$. 


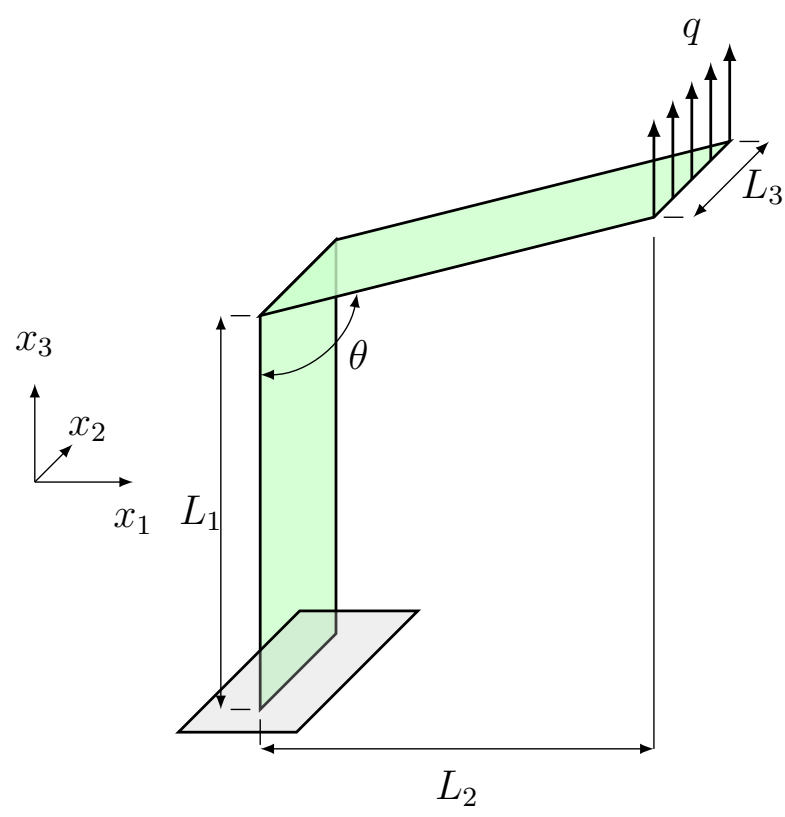

Figura 7.4: As dimensões e condições de contorno para a estrutura unida por um ângulo arbitrário $\theta[3]$.

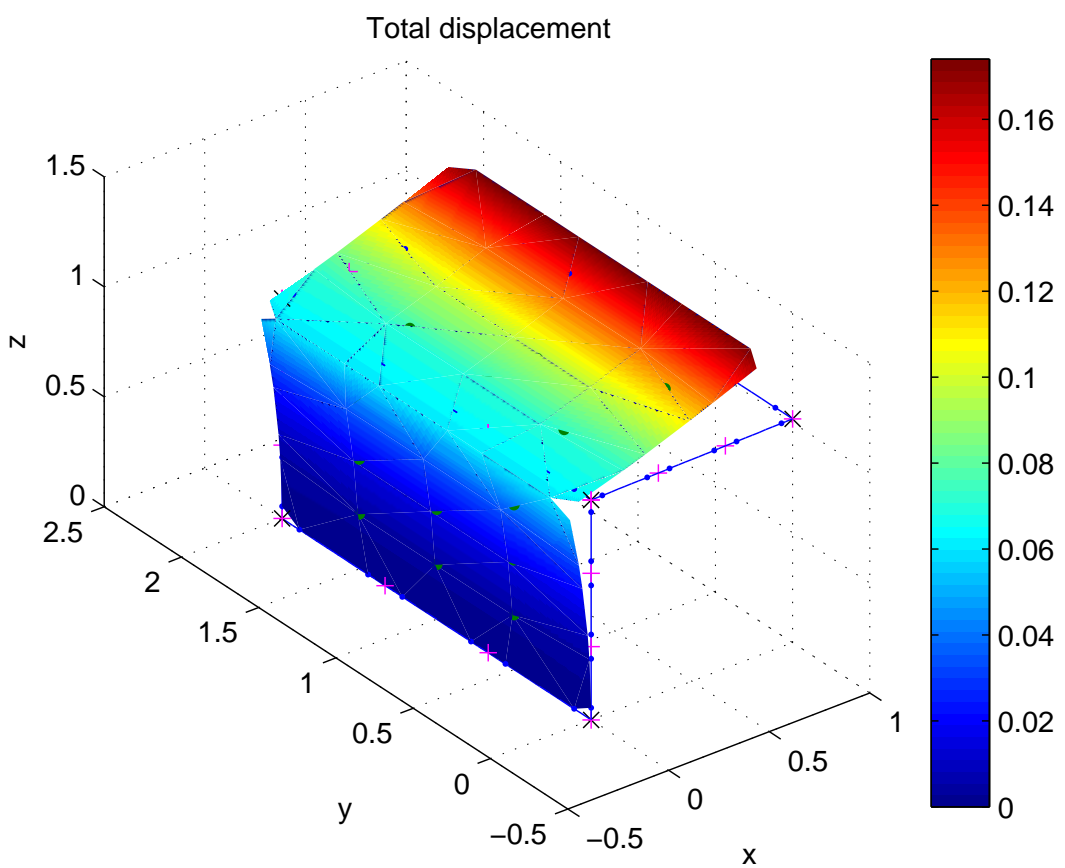

Figura 7.5: Deslocamento total para a estrutura unida por um ângulo $\theta=91^{\circ}$ obtido com o MEC convencional. 


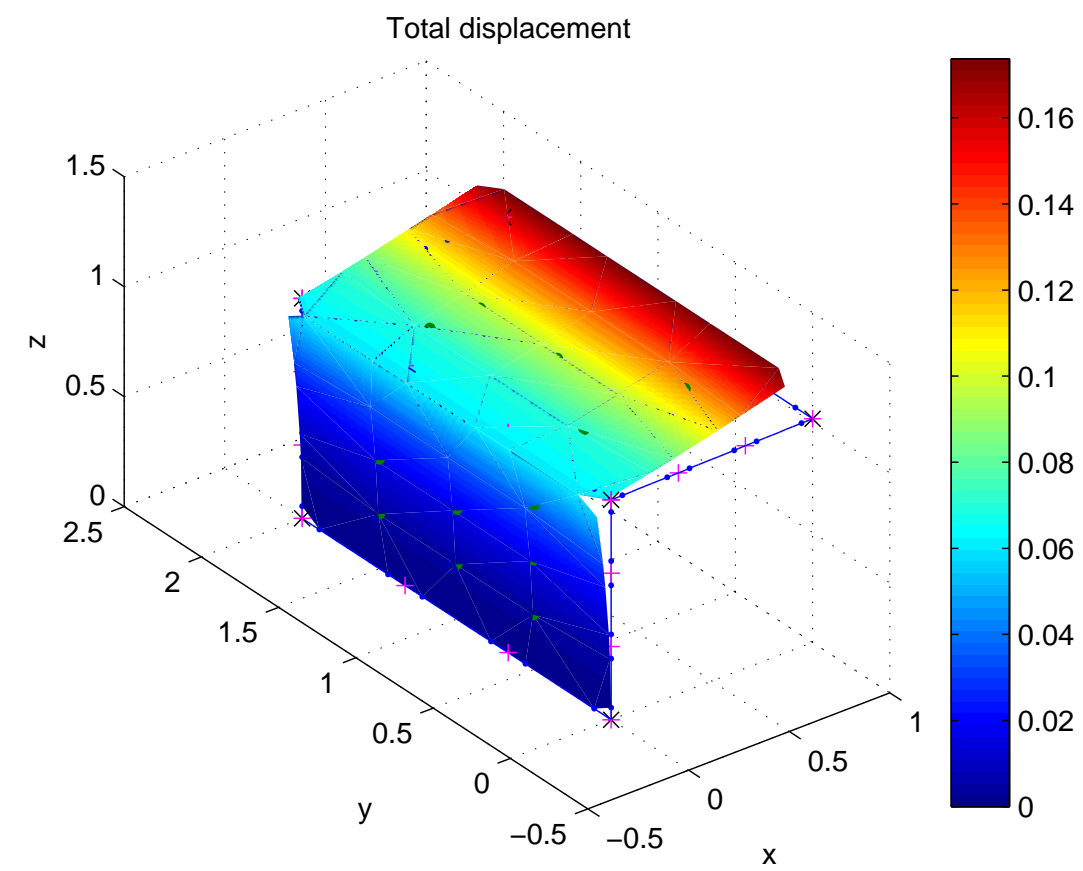

Figura 7.6: Deslocamento total para a estrutura unida por um ângulo $\theta=91^{\circ}$ obtido com a formulação deste trabalho.

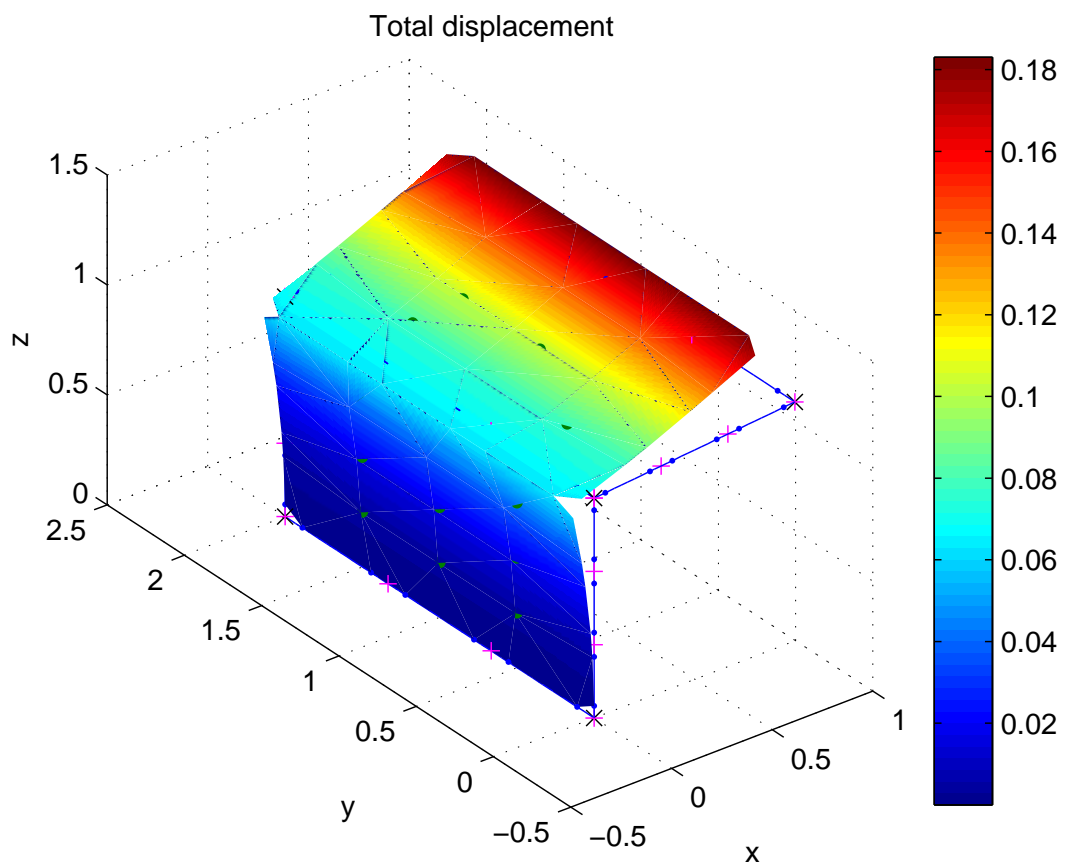

Figura 7.7: Deslocamento total para a estrutura unida por um ângulo $\theta=95^{\circ}$ obtido com o MEC convencional. 


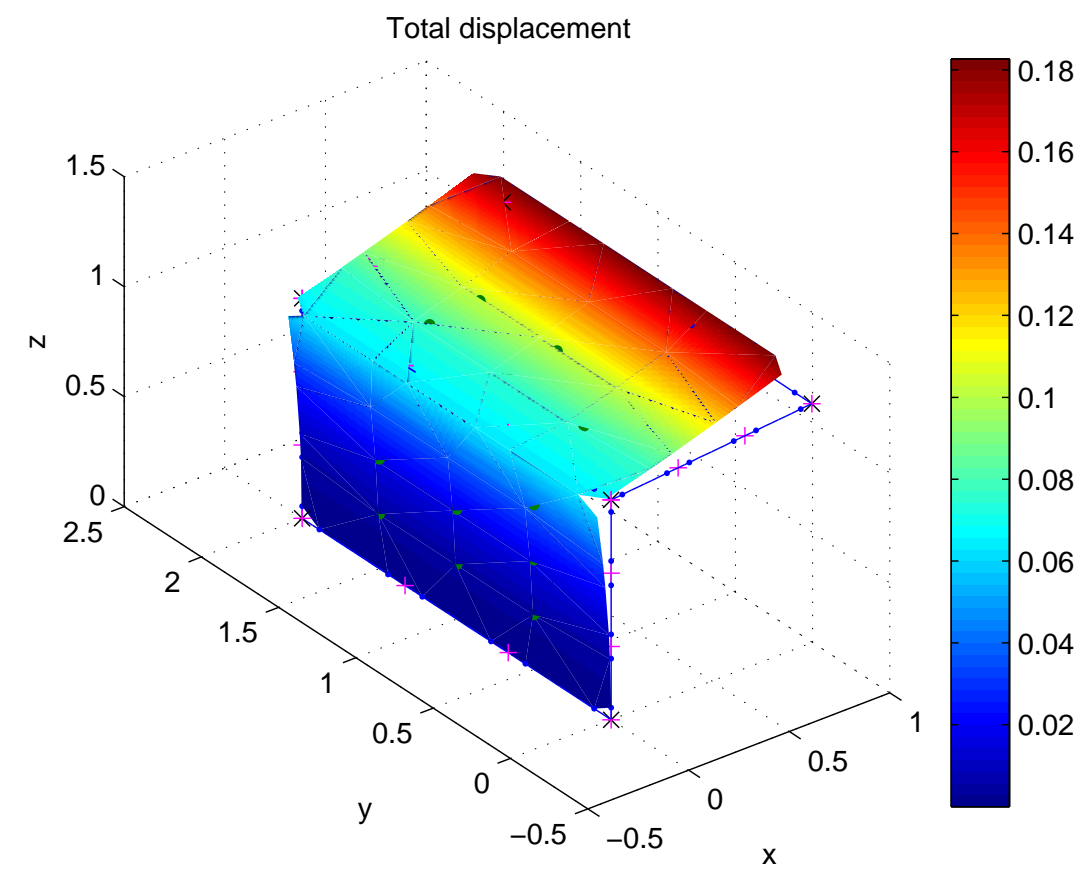

Figura 7.8: Deslocamento total para a estrutura unida por um ângulo $\theta=95^{\circ}$ obtido com a formulação deste trabalho.

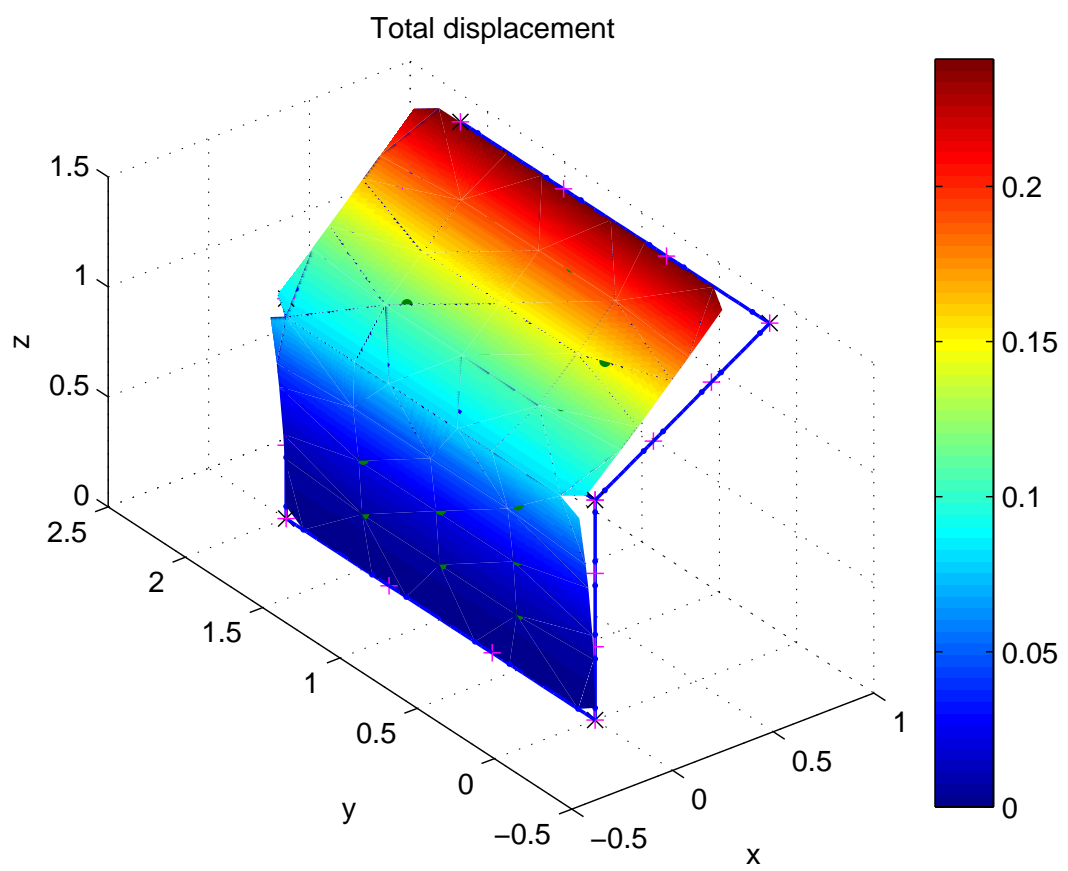

Figura 7.9: Deslocamento total para a estrutura unida por um ângulo $\theta=120^{\circ}$ obtido com o MEC convencional. 


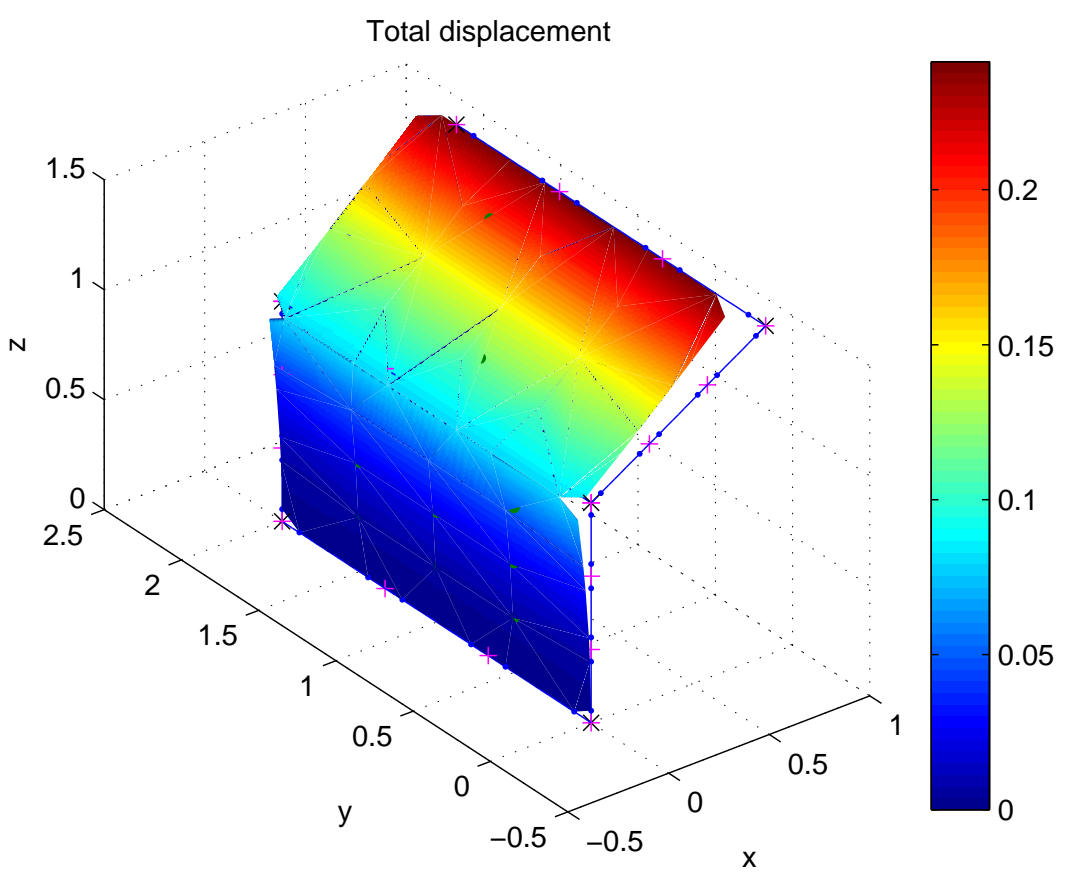

Figura 7.10: Deslocamento total para a estrutura unida por um ângulo $\theta=120^{\circ}$ obtido com a formulação deste trabalho.

Para este caso, uma pequena diferença entre os resultados da formulação deste trabalho e a convencional é observada. Como mencionado anteriormente não é esperado que haja diferença entre os resultados, uma vez que não há drilling rotation neste problema. A diferenças neste caso podem ser devidas a erros de truncamento. Os resultados para um ponto na extremidade carregada da placa superior são reunidos na Tabela 7.2. Também é mostrada uma comparação com as soluções analíticas de Bernoulli-Euler e Timoshenko. Os erros relativos são calculados e apresentados na Tabela 7.3. Todos os erros são inferiores a 1\% mostrando a precisão da simulação.

Tabela 7.2: Deslocamentos calculados para um ponto na extremidade carregada da placa superior comparados com a solução analítica para os três diferentes ângulos.

\begin{tabular}{cccc}
\hline Deslocamento, direção $x_{3}$ & $\theta=91^{\circ}$ & $\theta=95^{\circ}$ & $\theta=120^{\circ}$ \\
\hline MEC convencional & 0.1618 & 0.1654 & 0.1653 \\
MEC sem drilling rotation & 0.1620 & 0.1656 & 0.1654 \\
Solução de Bernouli-Euler & 0.1610 & 0.1646 & 0.1645 \\
Solução de Timoshenko & 0.1614 & 0.1650 & 0.1648 \\
\hline
\end{tabular}


Tabela 7.3: Erro calculado para cada uma das configurações em relação às teorias de viga de Bernouli-Euler e Timoshenko.

\begin{tabular}{cccc}
\hline & $\theta=91^{\circ}$ & $\theta=95^{\circ}$ & $\theta=120^{\circ}$ \\
\hline Solução de Bernouli Euler (\%) & 0.3712 & 0.6039 & 0.5139 \\
Solução de Timoshenko (\%) & 0.1237 & 0.3804 & 0.3869 \\
\hline
\end{tabular}

\subsection{Viga em formato de caixa}

Este modelo é formado por quatro placas dispostas de modo a se posicionarem como a parte lateral de uma caixa. As arestas localizadas no plano $\left(x_{1}-x_{3}\right)$ em $x_{2}=0$ possuem deslocamentos restritos em todas as direções. Os carregamentos são impostos como mostrado na Figura 7.11. A indicação das dimensões são dadas na mesma Figura, na qual $L_{1}=0.8 \mathrm{~m}, L_{2}=2 \mathrm{~m}, L_{3}=0.2 \mathrm{~m}, 0.1 \mathrm{~m}$ e $0.05 \mathrm{~m}$. A espessura das placas é $t_{1}=2.0 \mathrm{~mm}$. A carga distribuída ao longo das arestas indicadas na Figura 7.11 tem valor resultante $F=5000 N$. As propriedades mecânicas são $E=70 \mathrm{GPa}$ and $\nu=0.3$.

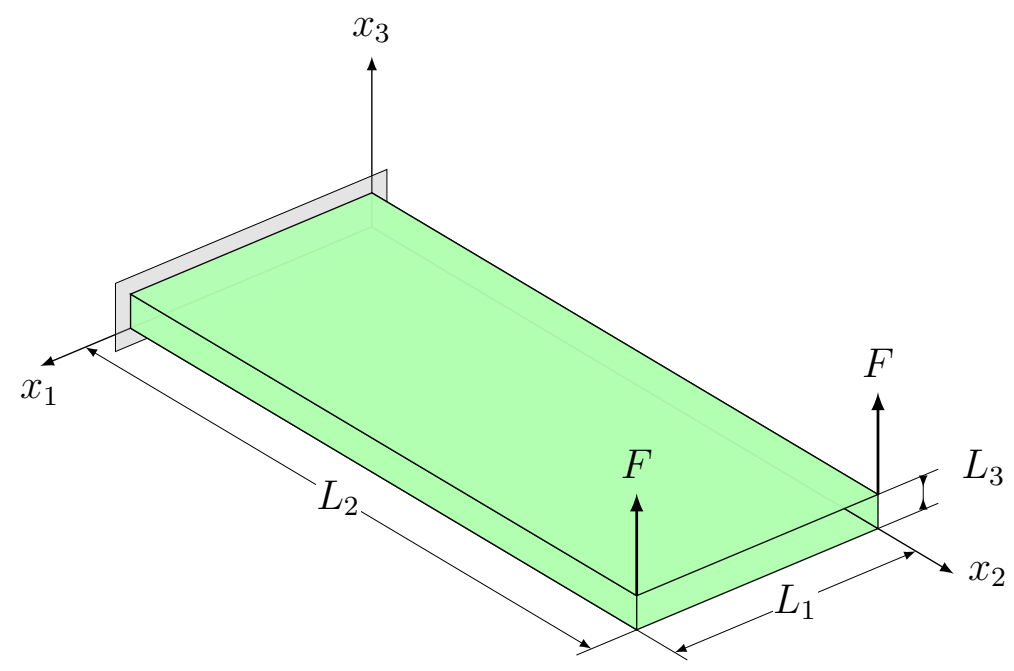

Figura 7.11: Dimensões e condições de contorno para a viga em forma de caixa em balanço.

O modelo foi discretizado usando quatro sub regiões, cada uma contendo 24 elementos de contorno lineares descontínuos. A Figura 7.12 apresenta a distribuição de deslocamento total na estrutura sujeita aos carregamentos indicados na Figura 7.11 para um valor de $L_{3}=0.1 \mathrm{~m}$. 


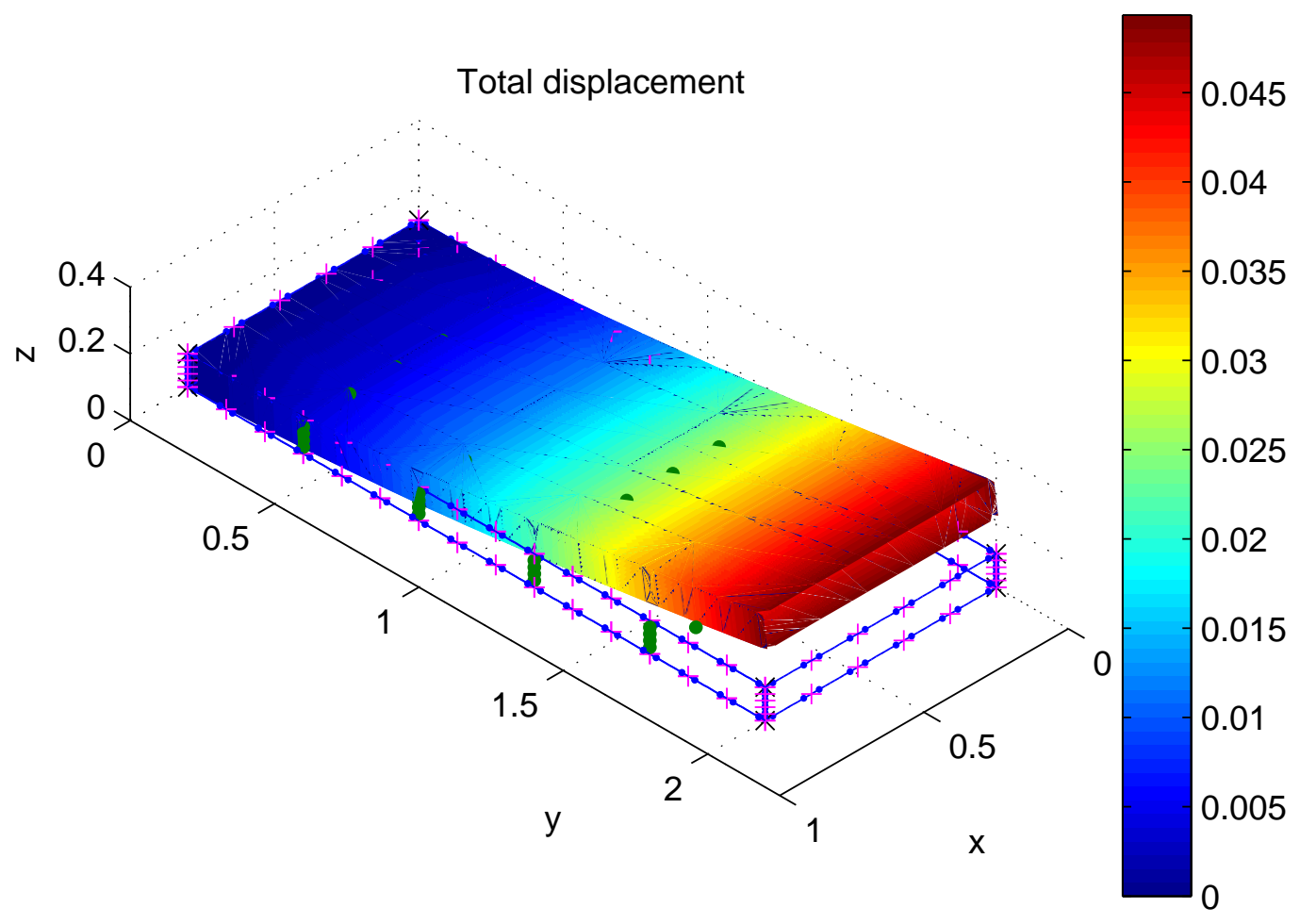

Figura 7.12: Deslocamento total para a viga em formato de caixa em balanço para $L_{3}=0.1 \mathrm{~m}$.

Os resultados nas Figuras 7.13, 7.14 e 7.15 são comparados com os resultados obtidos com o MEF (Ansys), a formulação convencional de associação de placas do MEC e a solução analítica de Bernoulli-Euler. O plano $x_{2}-x_{3}$ apresentado nas Figuras a seguir está posicionado em $x_{1}=0.8 \mathrm{~m}$. 


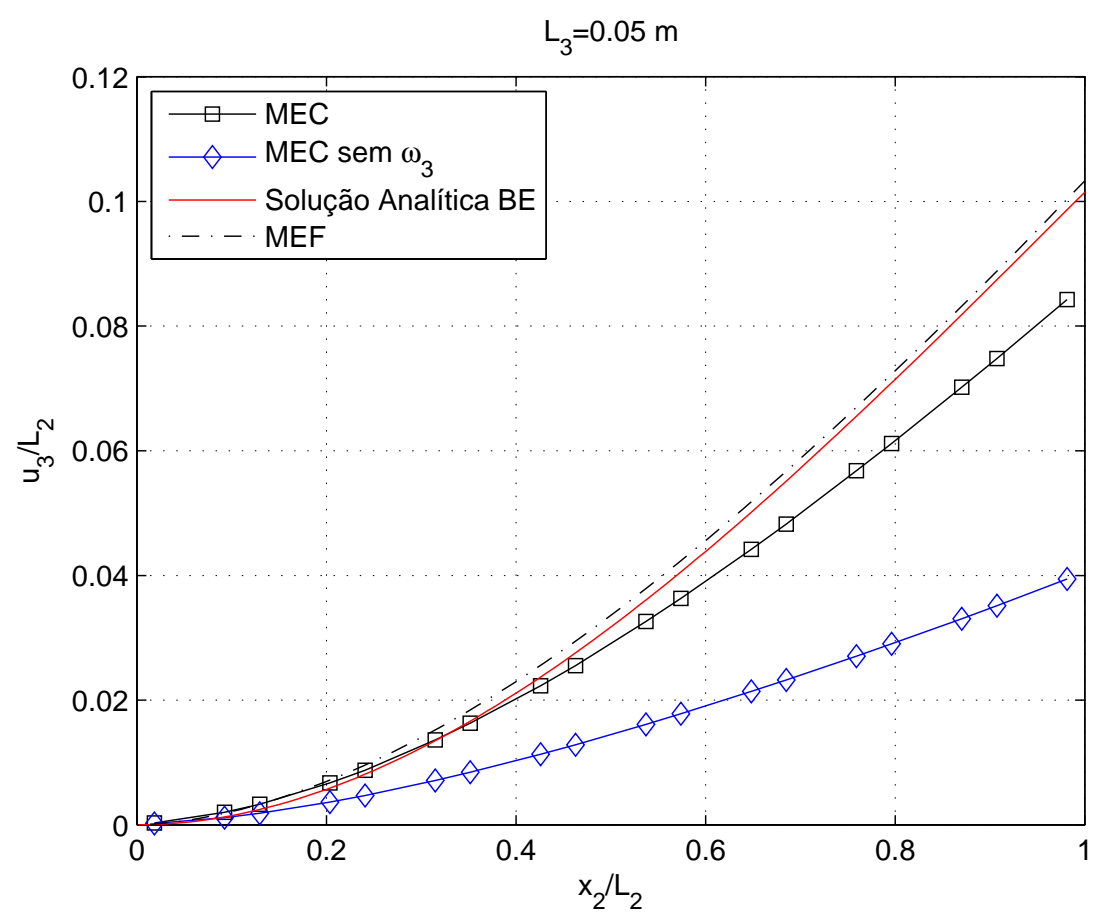

Figura 7.13: Deslocamento na direção $x_{3}$ para a viga em formato de caixa em balanço para $L_{3}=0.05 \mathrm{~m}$.

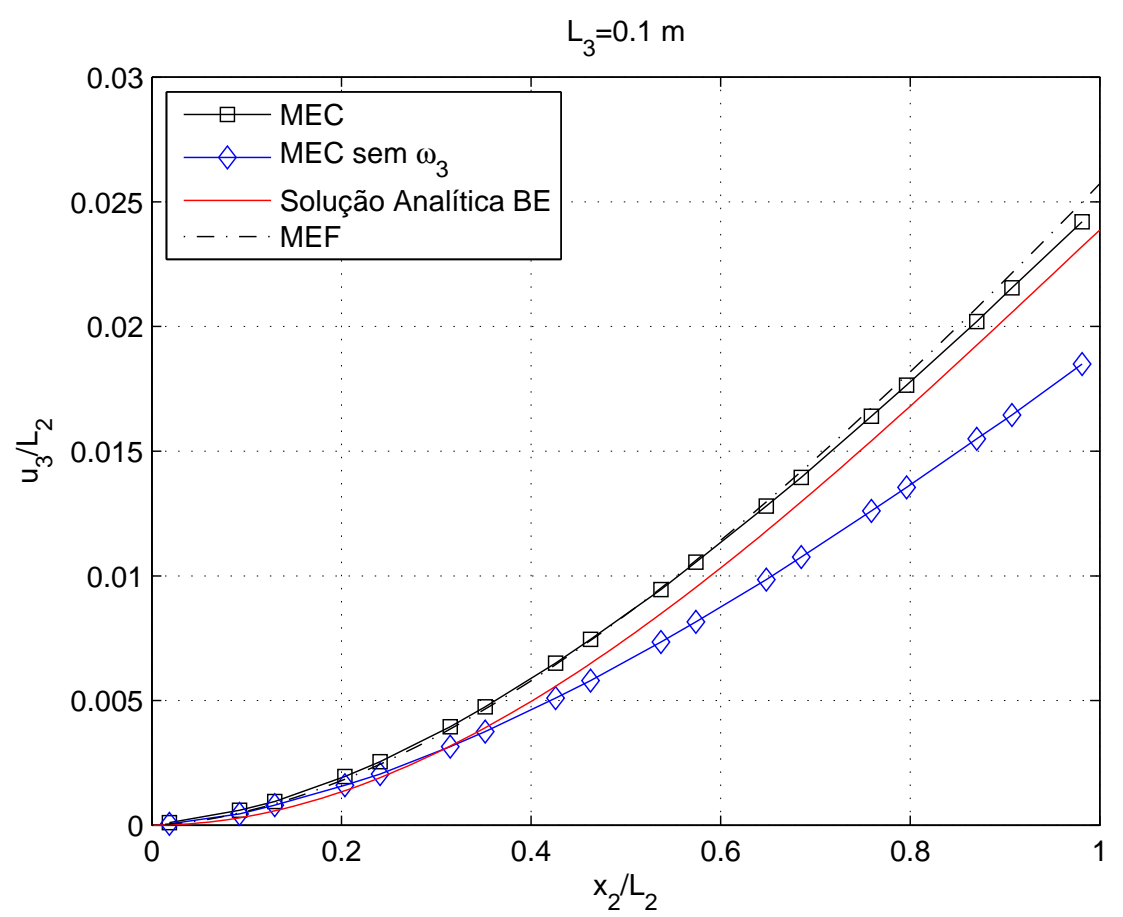

Figura 7.14: Deslocamento na direção $x_{3}$ para a viga em formato de caixa em balanço para $L_{3}=0.1 \mathrm{~m}$. 


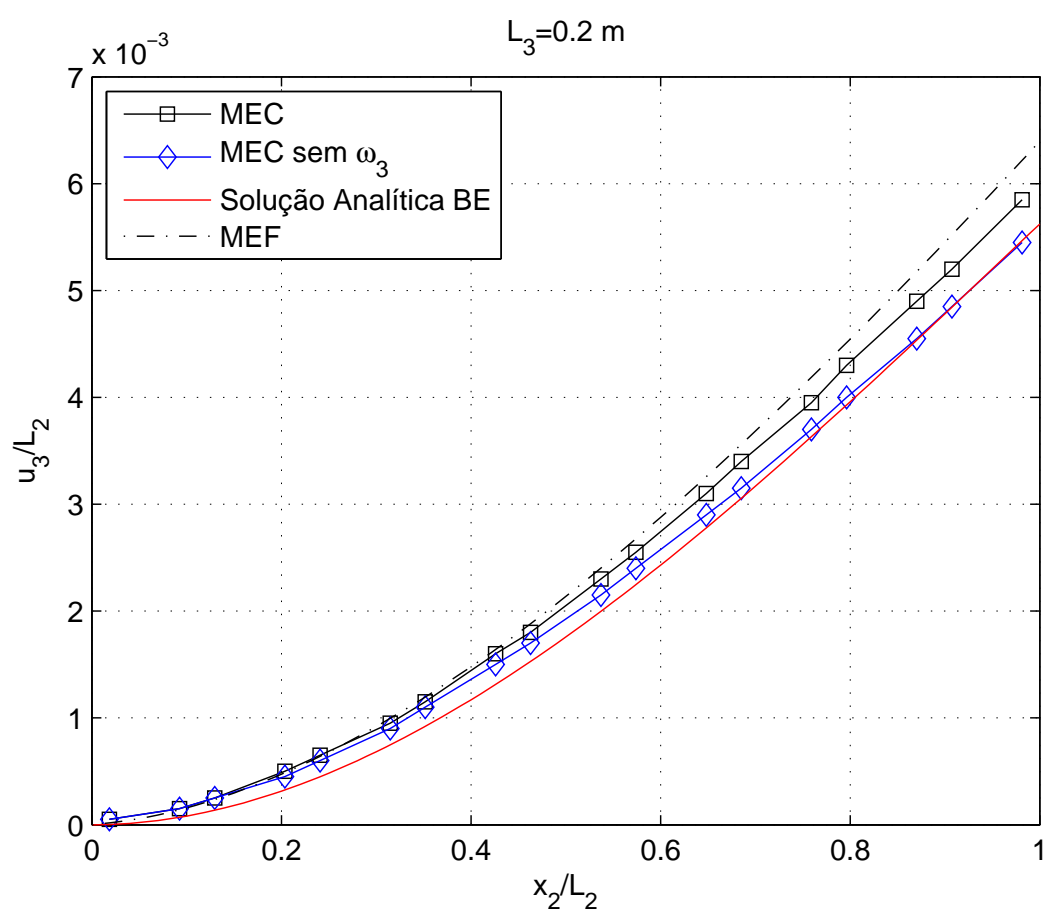

Figura 7.15: Deslocamento na direção $x_{3}$ para a viga em formato de caixa em balanço para $L_{3}=0.2 \mathrm{~m}$.

O acordo dos resultados com a solução analítica parece estar relacionado à razão de aspecto da caixa $\left(L_{2} / L_{3}\right)$. Com seu aumento, as duas soluções, a deste trabalho e a convencional, desviam dos resultados obtidos com o MEF (Ansys). Mesmo assim, quando comparados com os resultados da formulação convencional, o presente trabalho apresenta um melhor acordo.

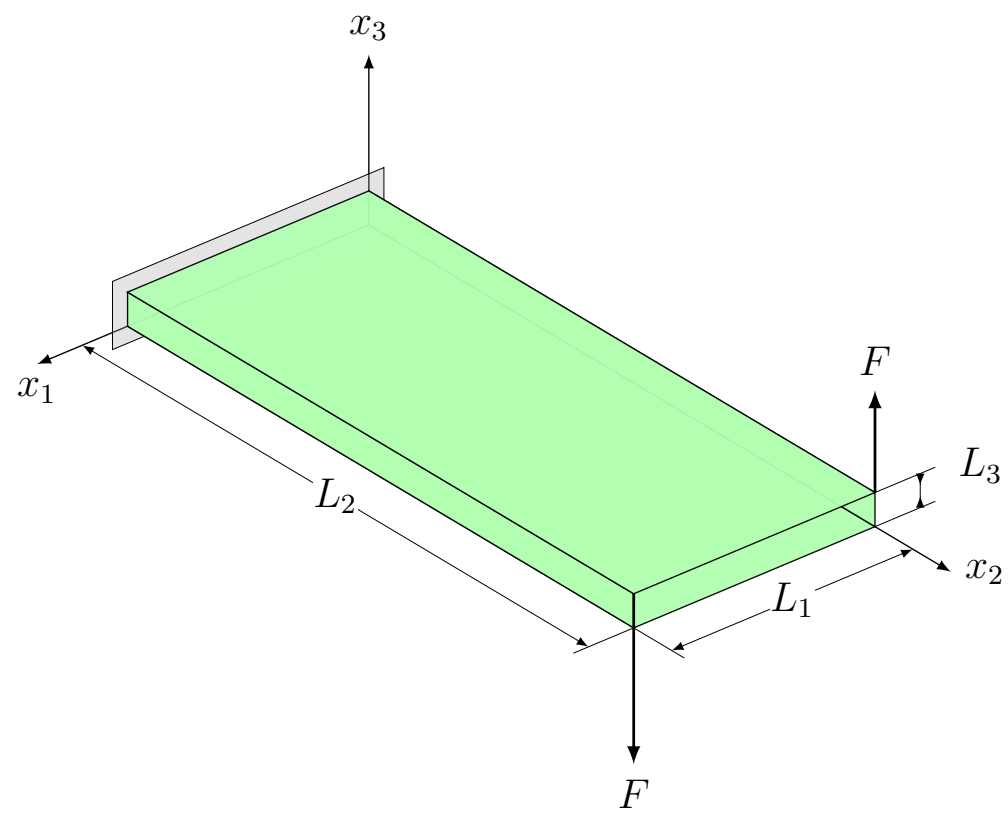

Figura 7.16: Dimensões e condições de contorno para a viga em forma de caixa em balanço sujeita a um momento em sua extremidade livre. 
A Figura 7.16 apresenta a mesma geometria porém com os carregamentos impostos de maneira a gerar um binário em sua extremidade livre. A Figura 7.17 apresenta a distribuição do deslocamento total na viga caixa sujeita aos carregamentos descritos. Os Resultados nas Figuras 7.18, 7.19 e 7.20 são comparados àqueles obtidos com o MEF (Ansys) e a formulação convencional do MEC para associação de placas.

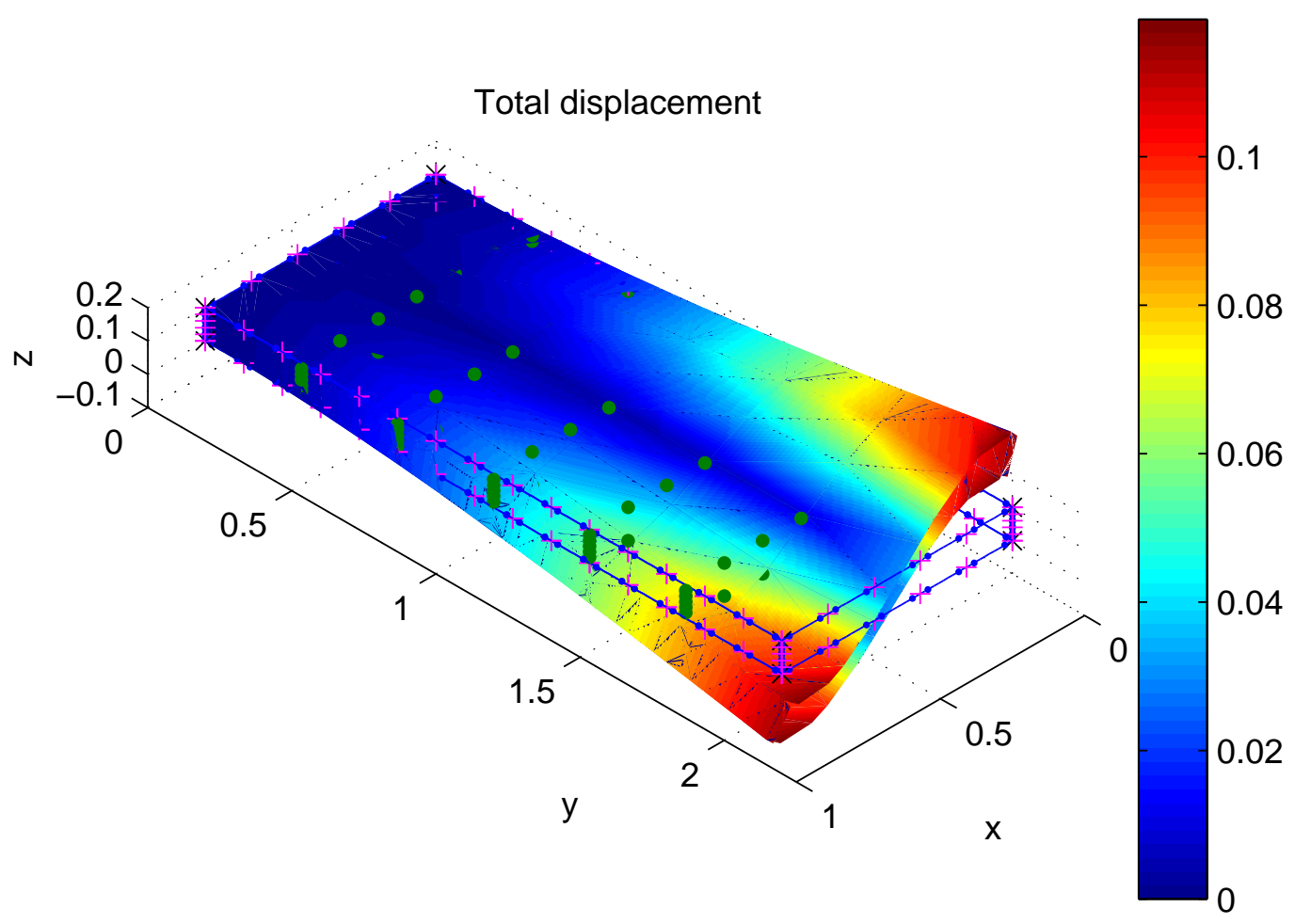

Figura 7.17: Deslocamento total para a viga em formato de caixa em balanço sujeita a um momento para $L_{3}=0.1 \mathrm{~m}$.

Os deslocamentos calculados para $L_{3}=0.05 \mathrm{~m}$ são muito elevados causando uma interferência da placa inferior com a placa superior. Estes resultados, apesar do acordo obtido, não possuem significado físico. Para $L_{3}=0.1 \mathrm{~m}$, os presentes resultados estão em bom acordo com aqueles obtidos com o MEF (Ansys) mas o MEC convencional mostra um comportamento diferente, resultando em deslocamentos menores. Finalmente, para $L_{3}=0.2 \mathrm{~m}$ a influência da inclusão da drilling rotation parece ser negligenciável. 


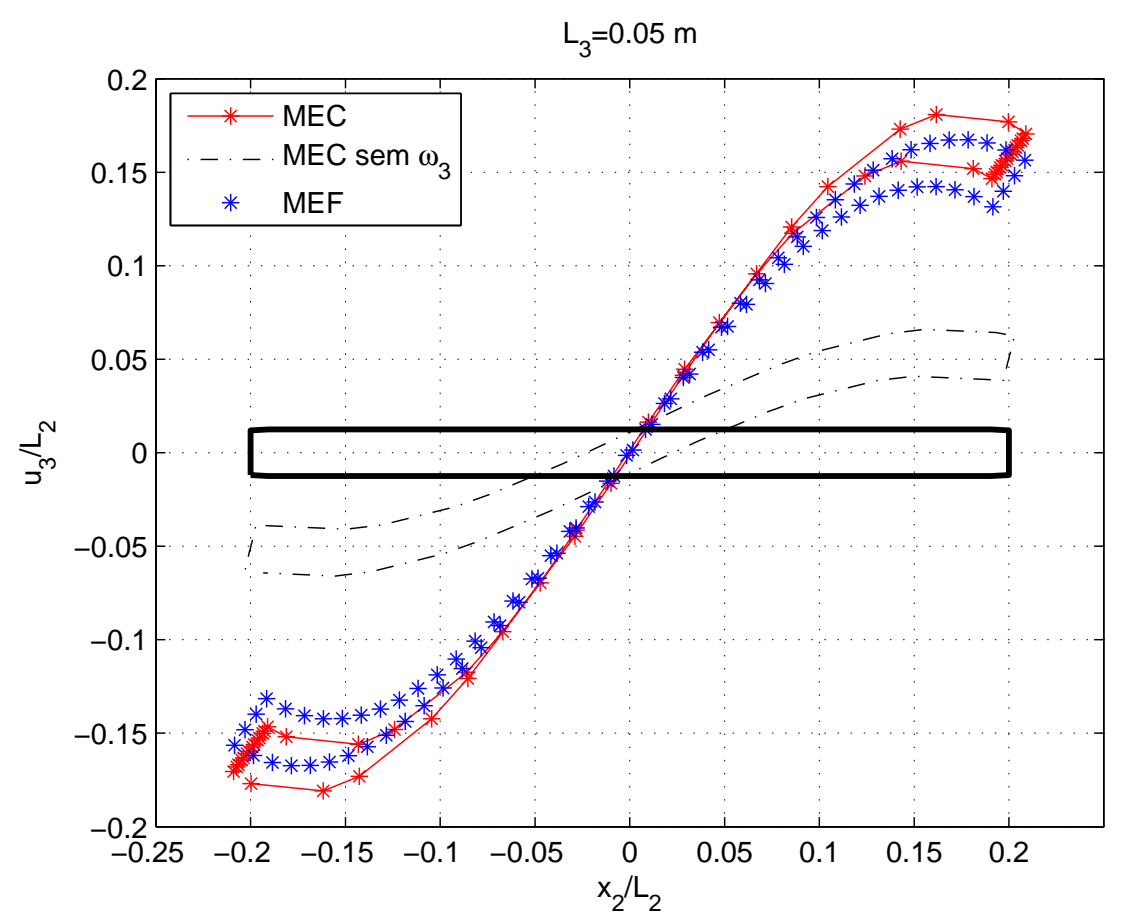

Figura 7.18: Deslocamentos em $x_{2}=2 \mathrm{~m}$ para a viga em formato de caixa em balanço sujeita a um momento para $L_{3}=0.05 \mathrm{~m}$.

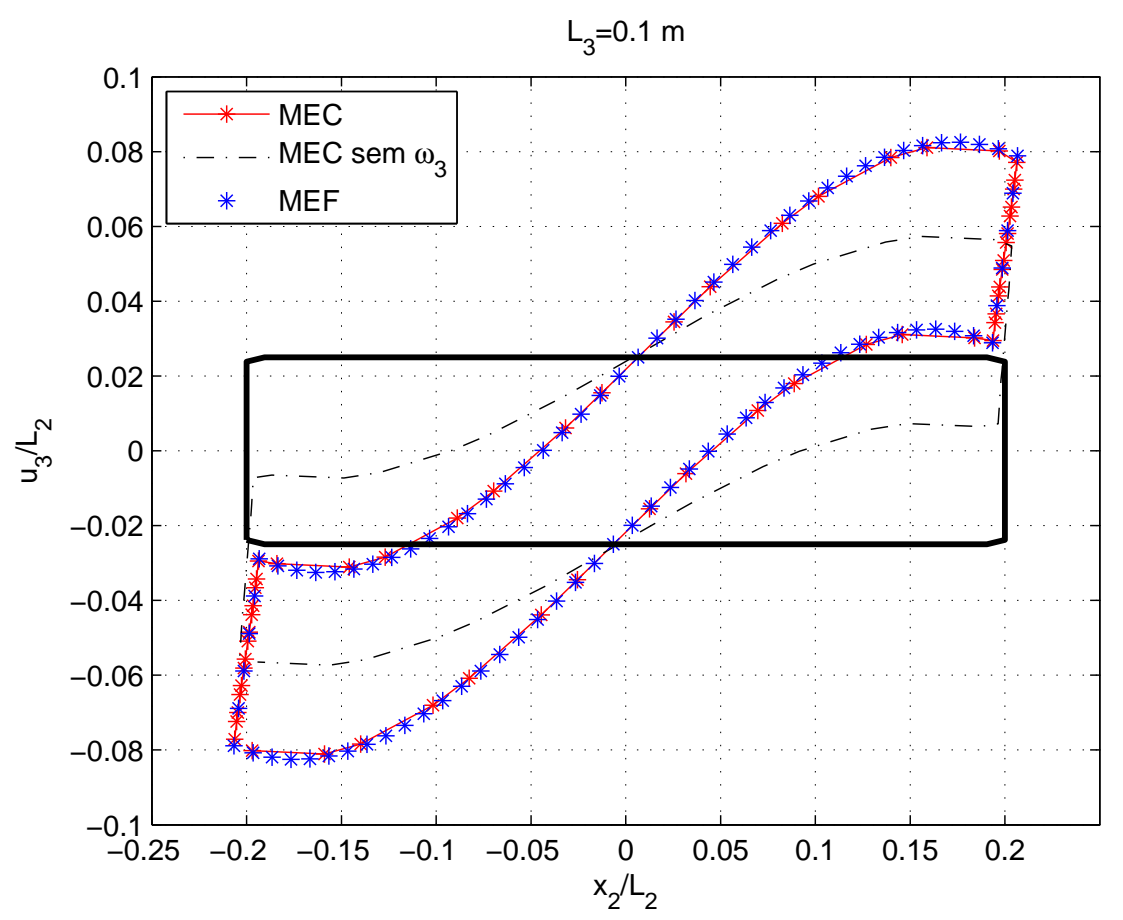

Figura 7.19: Deslocamentos em $x_{2}=2 \mathrm{~m}$ para a viga em formato de caixa em balanço sujeita a um momento para $L_{3}=0.1 \mathrm{~m}$. 


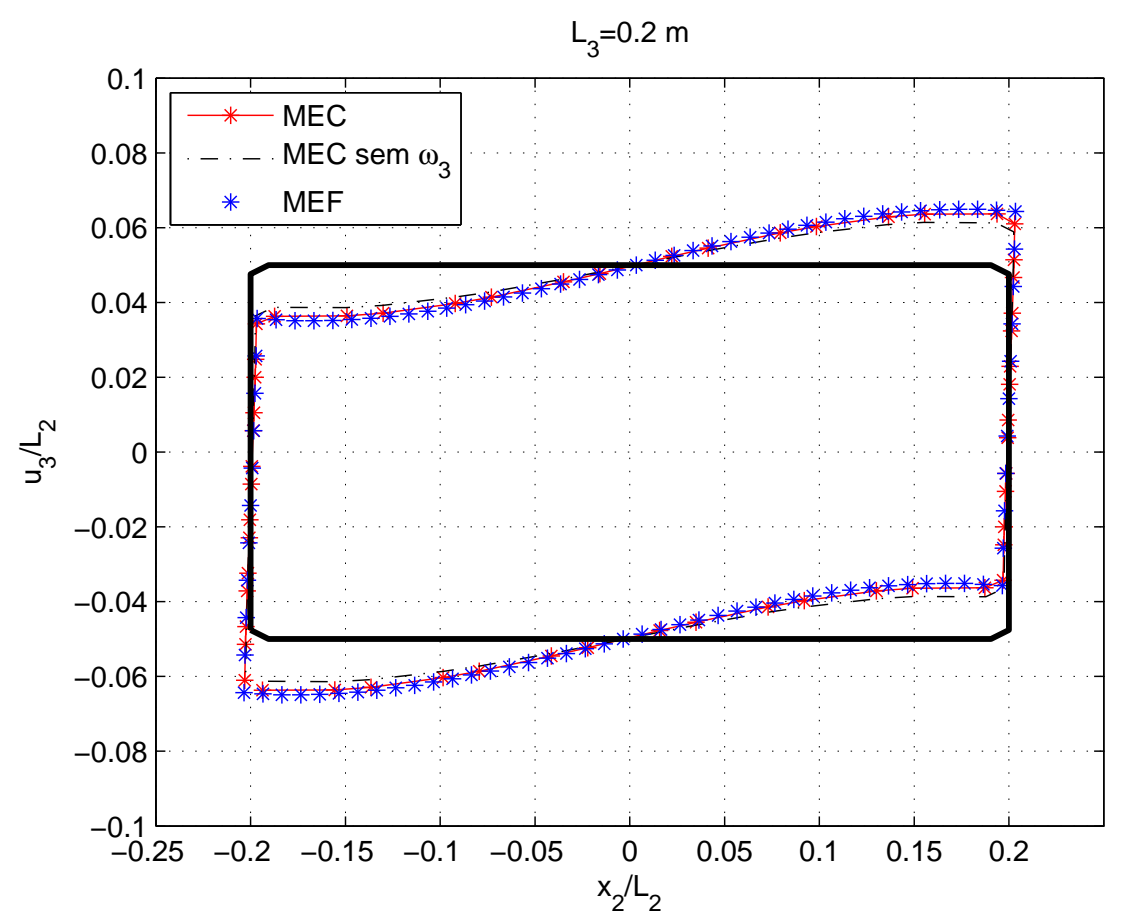

Figura 7.20: Deslocamentos em $x_{2}=2 \mathrm{~m}$ para a viga em formato de caixa em balanço sujeita a um momento para $L_{3}=0.2 \mathrm{~m}$. 


\subsubsection{Resultados usando elementos quadráticos}

O problema com os carregamentos indicados na Figura 7.11, foi resolvido utilizando também elementos quadráticos com 24 elementos por subregião. Uma malha mais refinada com a interpolação linear foi também incluída, com 40 elementos por sub região. As Figuras 7.21, 7.22 e 7.23 mostram o plano $x_{2}-x_{3}$ posicionado em $x_{1}=0.8 \mathrm{~m}$.

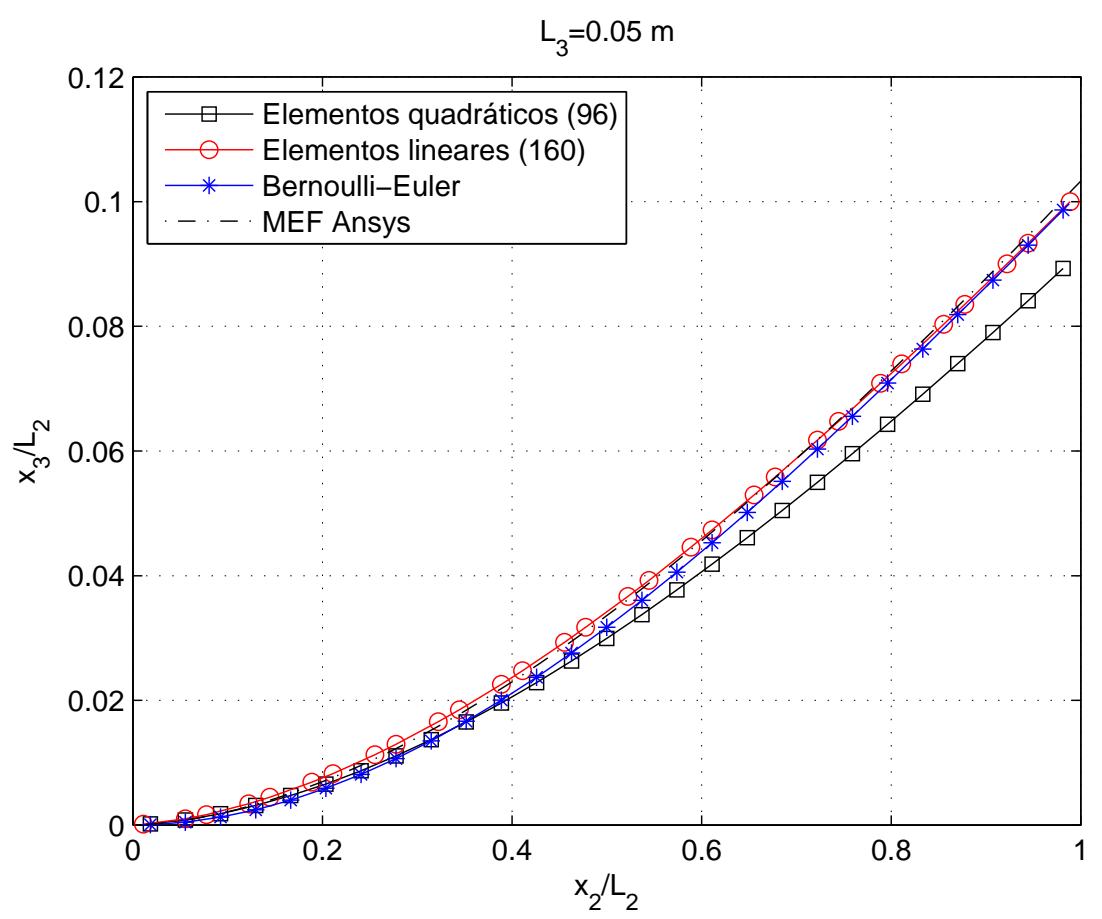

Figura 7.21: Deslocamentos $u_{2}$ para o lado alinhado com a direção $x_{2}$ localizados nas coordenadas $x_{1}=x_{3}=0 \mathrm{~m}$ para $L_{3}=0.05 \mathrm{~m}$. O número de elementos das simulações é indicado na legenda.

A Tabela 7.4 apresenta o erro relativo dos resultados quando comparados à solução analítica de Bernoulli-Euler (BE).

Tabela 7.4: Deslocamentos $u_{2}$ máximos obtidos com o MEC comparados à solução analítica de Bernoulli-Euler.

\begin{tabular}{ccccccc}
\hline$L_{3}$ & $0.2 \mathrm{~m}$ & Erro (\%) & $0.1 \mathrm{~m}$ & Erro (\%) & $0.05 \mathrm{~m}$ & Erro (\%) \\
\hline Linear & 0.011892 & 9.1 & 0.049388 & 6.44 & 0.20426 & 3.48 \\
Quadrático & 0.011716 & 7.49 & 0.046772 & 0.8 & 0.18463 & 6.47 \\
BE & 0.0109 & & 0.0464 & & 0.1974 & \\
\hline
\end{tabular}

O refinamento da malha e a alteração da função de forma melhoraram o acordo quando comparado a resposta anterior. 


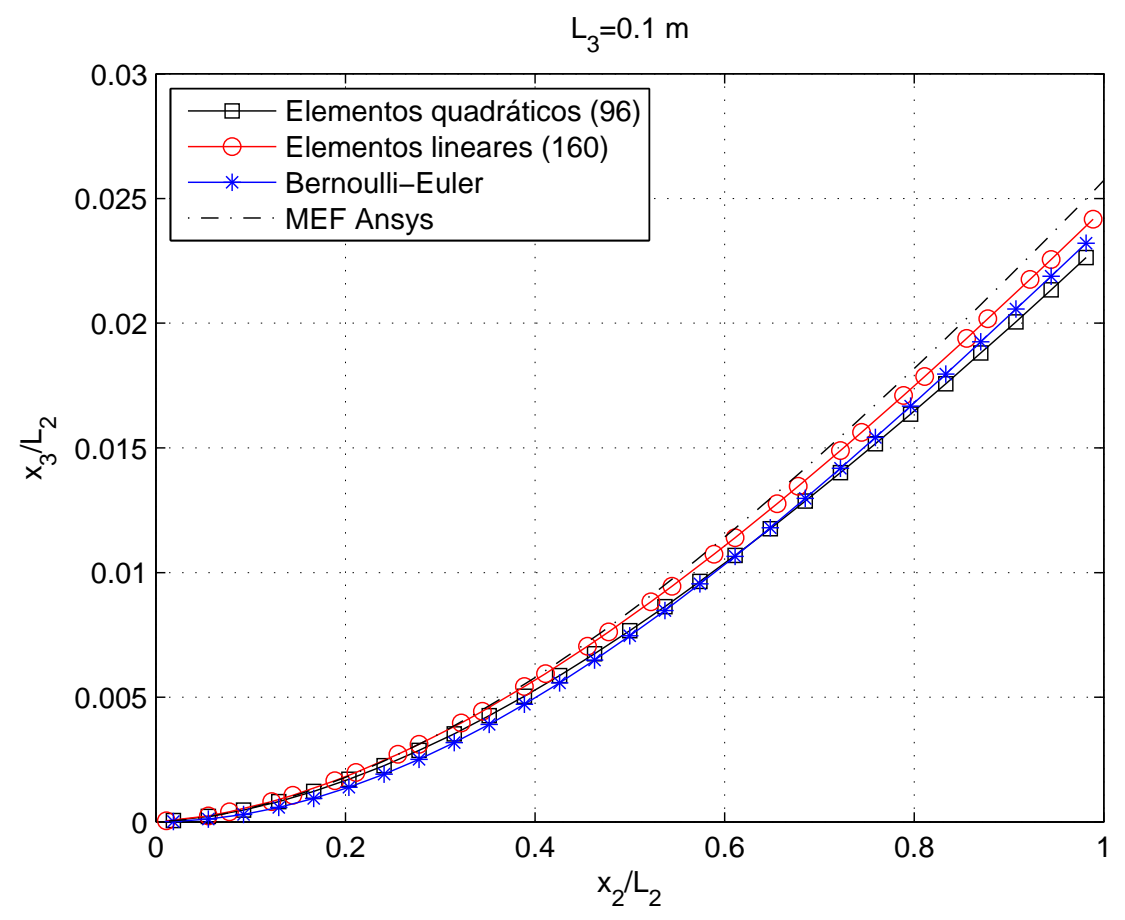

Figura 7.22: Deslocamentos $u_{2}$ para o lado alinhado com a direção $x_{2}$ localizados nas coordenadas $x_{1}=x_{3}=0 \mathrm{~m}$ para $L_{3}=0.1 \mathrm{~m}$. O número de elementos das simulações é indicado na legenda.

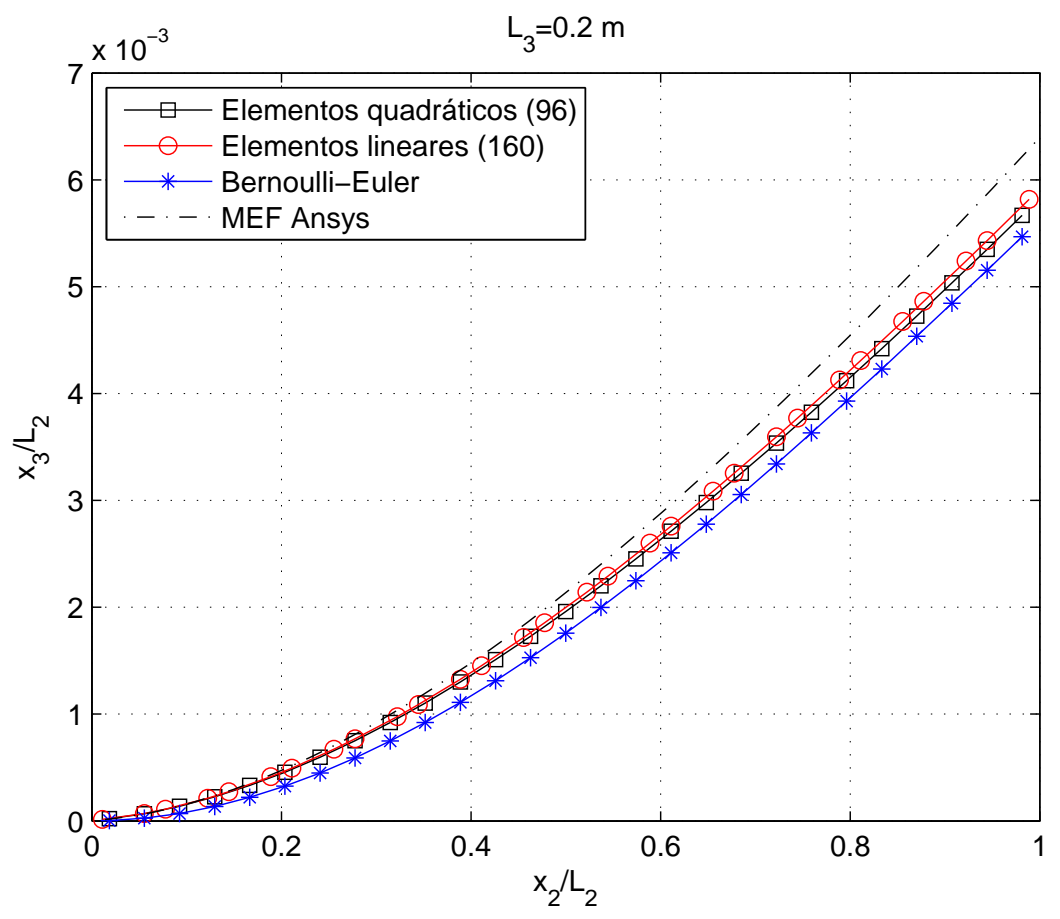

Figura 7.23: Deslocamentos $u_{2}$ para o lado alinhado com a direção $x_{2}$ localizados nas coordenadas $x_{1}=x_{3}=0 \mathrm{~m}$ para $L_{3}=0.2 \mathrm{~m}$. O número de elementos das simulações é indicado na legenda. 


\subsection{Associação em L com carregamento lateral}

Este problema foi simulado com o intuito de avaliar o caso em que a rotação $\omega_{3}$ é relevante no que diz respeito à imposição das condições de compatibilidade. Este modelo foi discretizado usando duas sub regiões, cada uma contendo 60 elementos de contorno lineares descontínuos. As propriedades mecânicas, dimensões e carregamentos são dados na Figura 7.24. Como é possível observar, o carregamento colocado na lateral impõe uma rotação ao redor do eixo $x_{3}$, a mesma direção da rotação $\omega_{3}$ da placa superior. Os resultados são apresentados nas Figuras 7.25 para o MEC com rotação incluída, 7.26 para o MEC convencional e 7.27 para o MEF.

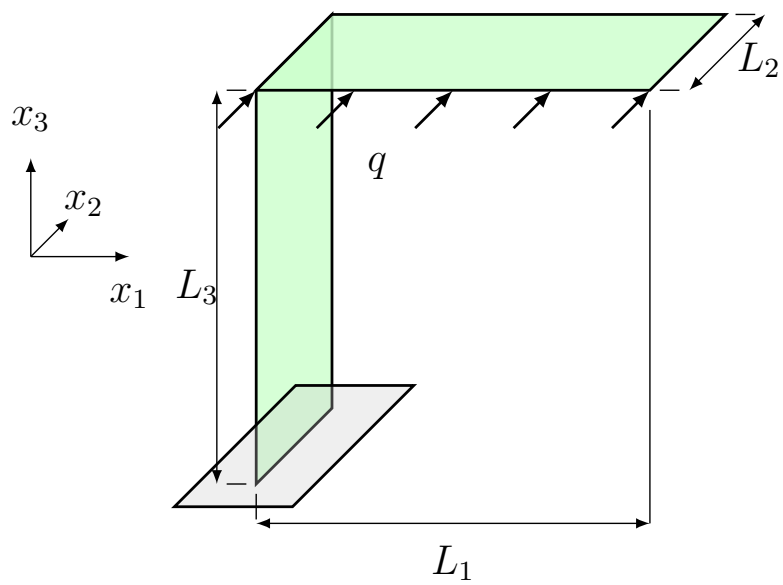

Figura 7.24: L-shaped structure, $L_{1}=1.0 \mathrm{~m}, L_{2}=2.0 \mathrm{~m}, L_{3}=1.0 \mathrm{~m}, t=0.1 \mathrm{~m}$ (espessura da placa). $E=100 \mathrm{kPa}, \nu=0$ e $q=10 \mathrm{~N}$.

Para que seja possível comparar os resultados obtidos com a formulação do MEC deste trabalho, MEC convencional e MEF (Ansys), a Figura 7.28 é apresentada. Este gráfico mostra os deslocamentos nas direções $x_{1}$ e $x_{2}$ para a placa superior, projetados no plano $x_{1}-x_{2}$. Indica claramente que os resultados obtidos com a formulação apresentada neste trabalho tem um melhor acordo com a solução obtida com MEF (Ansys). A formulação convencional produziu valores que são uma ordem de magnitude menores (Figura 7.26). 


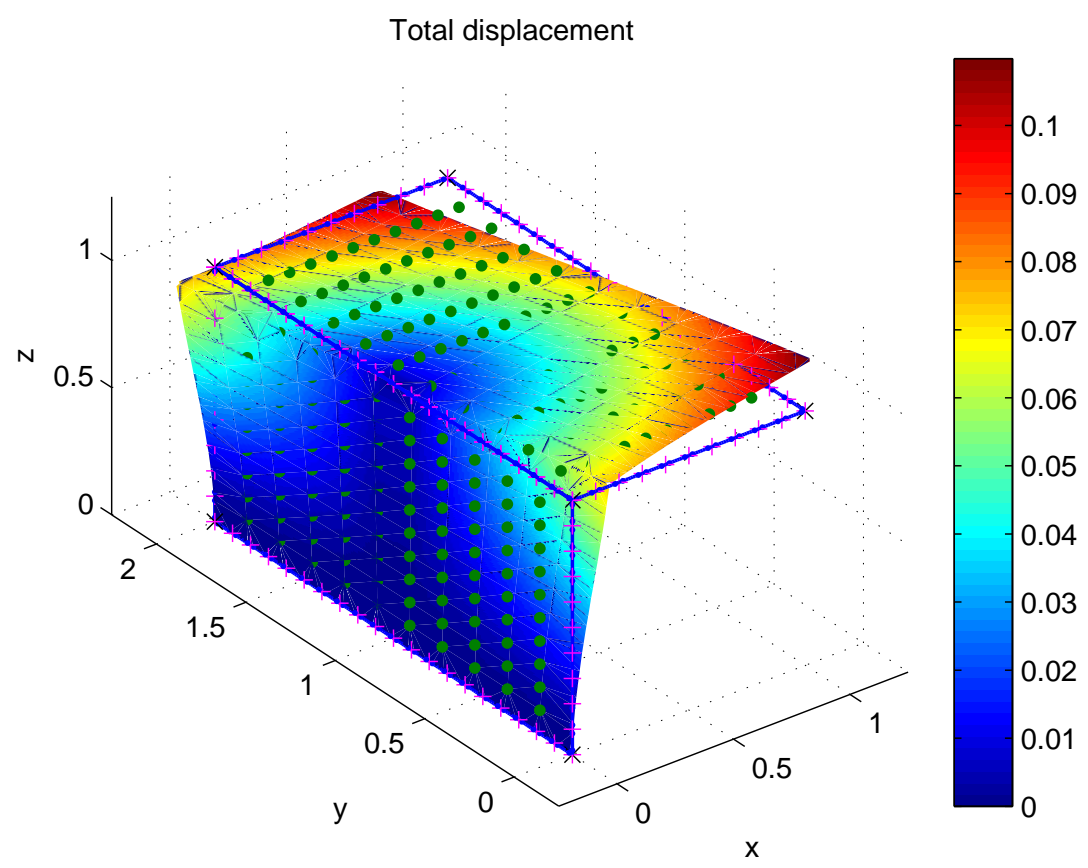

Figura 7.25: Resultados obtidos com a formulação do MEC apresentada nesta tese.

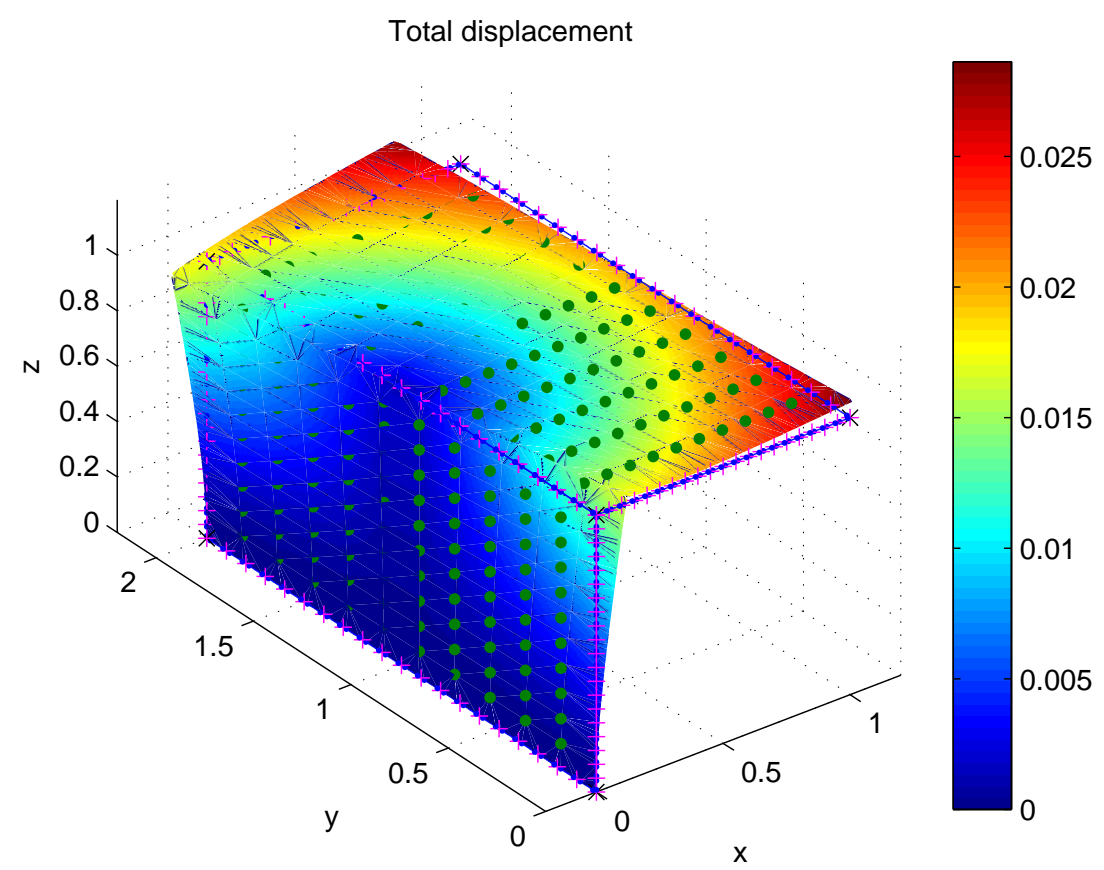

Figura 7.26: Resultados obtidos com a formulação do MEC convencional. 


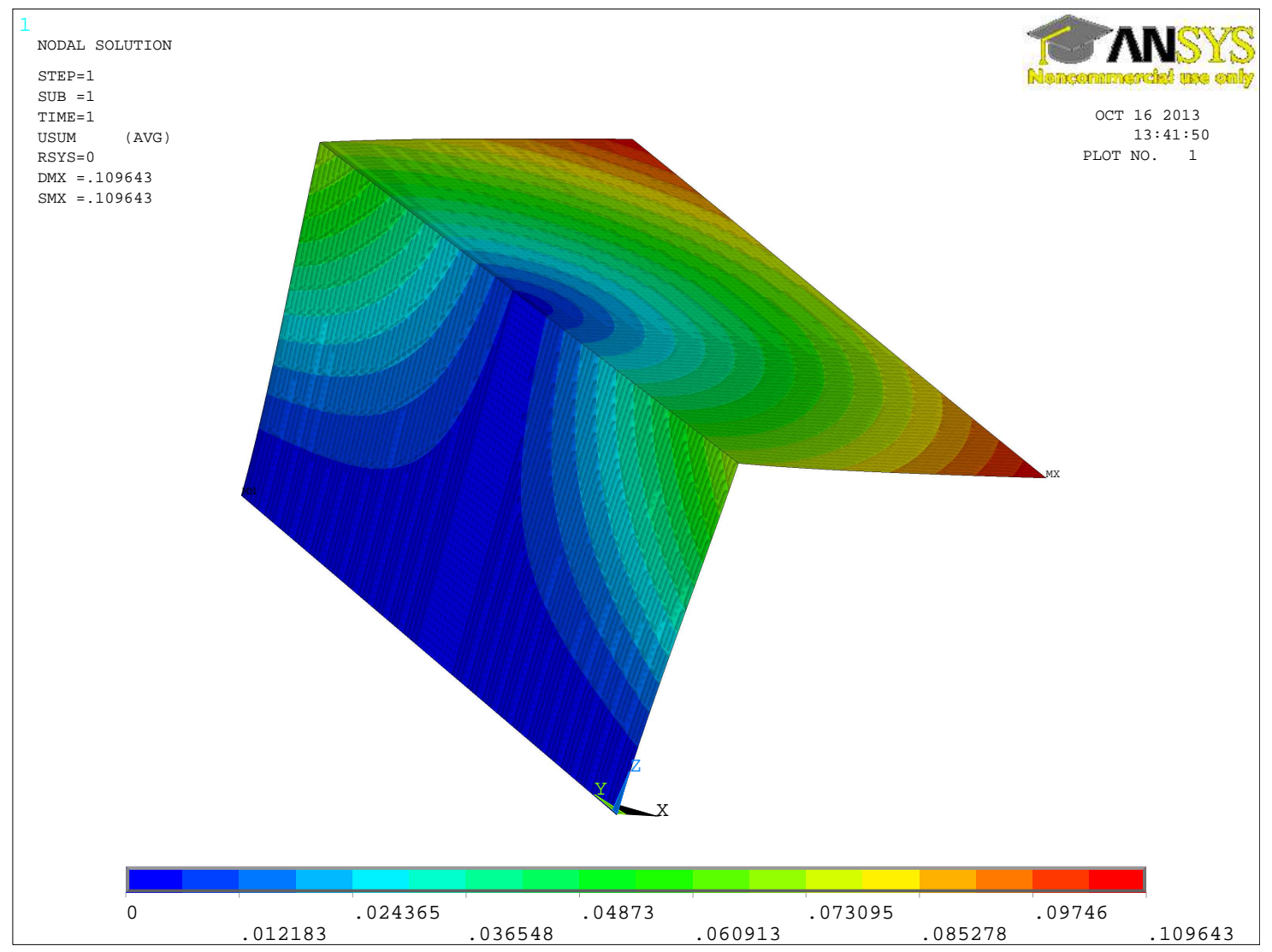

Figura 7.27: Resultados obtidos usando o MEF através do ANSYS com o elemento Shell181.

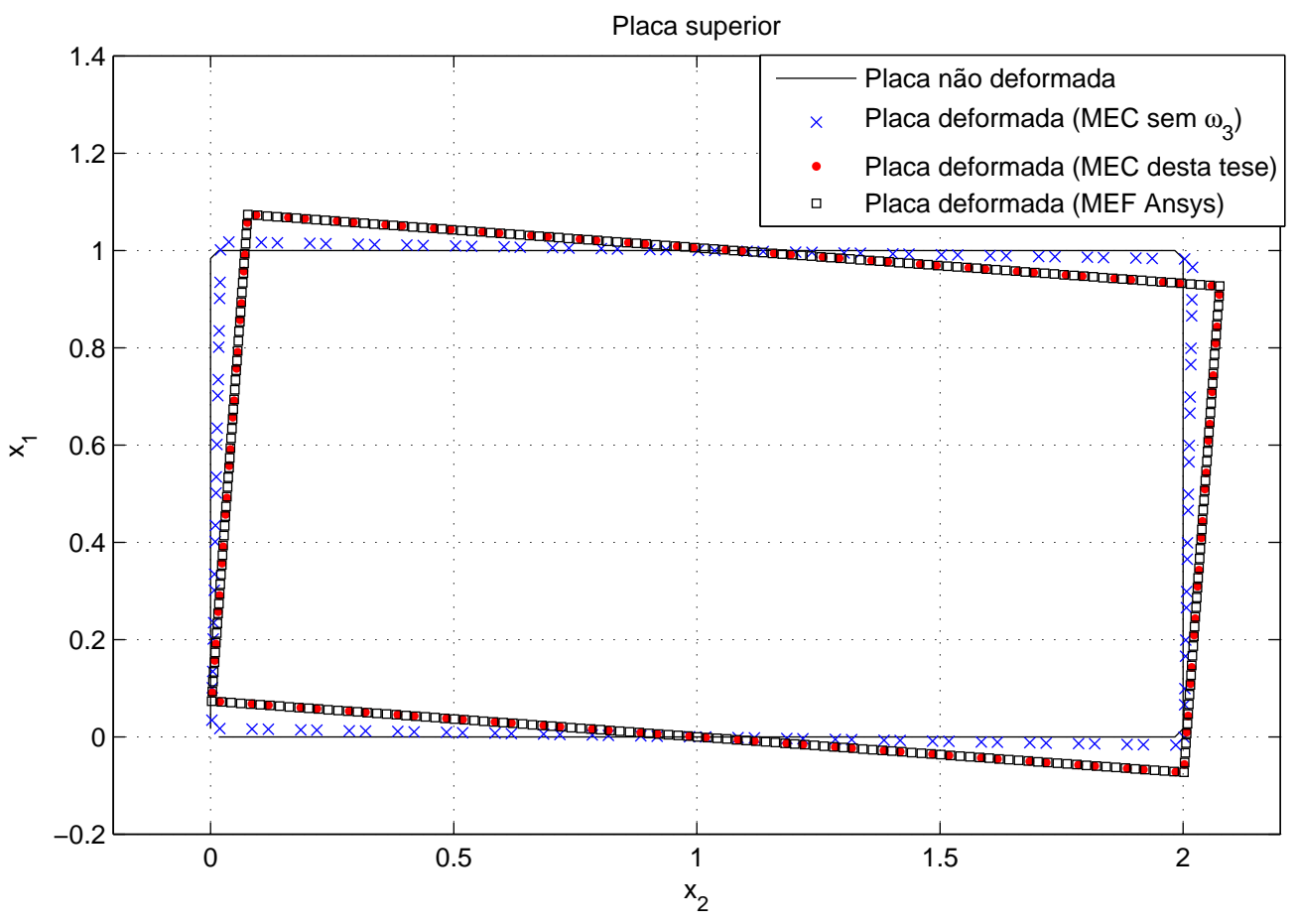

Figura 7.28: Resultados para a placa superior projetados no plano $x_{1}-x_{2}$. 


\section{CONSIDERAÇÕES FINAIS}

\subsection{Conclusões}

Nesta tese uma nova formulação do MEC foi desenvolvida para a análise de estruturas formadas pela associação tridimensional de placas espessas. A abordagem apresentada, contém seis graus de liberdade por nó, três deslocamentos e três rotações. Ela foi construída a partir da associação das formulações de elasticidade plana e placas deformáveis por cisalhamento. A primeira delas sendo modificada para incluir a rotação em torno do eixo $x_{3}$, perpendicular ao plano da placa. Esta rotação foi incluída com a aplicação da equação integral de contorno da elasticidade plana na expressão analítica da rotação, oriunda da teoria da elasticidade. No arranjo tridimensional, cada placa foi definida como uma sub região e os termos locais foram calculados de maneira independente em coordenadas locais. As condições de compatibilidade de deslocamentos e rotações, e equilíbrio de momentos e forças de superfície foram impostas desconsiderando as restrições comumente adotadas em formulações do MEC. Finalmente, o sistema global foi montado, as condições de contorno impostas, resolvendo-o por eliminação gaussiana. Vários testes numéricos foram utilizados para validação. Inicialmente para problemas de elasticidade plana, com o intuito de verificação da precisão das rotações obtidas. Depois problemas com associação de placas foram avaliados de maneira a verificar o efeito das modificações propostas. Os resultados foram comparados com soluções analíticas e aqueles obtidos com o método dos elementos finitos.

A rotação $\omega_{3}$ também conhecida como drilling rotation foi calculada a partir de uma expressão exata, dada por uma equação integral de contorno. Os resultados foram avaliados através da comparação com vários trabalhos disponíveis na literatura para o caso da elasticidade plana. Uma comparação foi também realizada com a formulação previamente desenvolvida, que utilizou conceitos de partição da unidade e um elemento enriquecido pela rotação em questão. Os resultados deste trabalho foram consistentemente melhores quando comparados às soluções analíticas e resultados obtidos com elementos finitos. Para o caso da viga fina, a convergência foi problemática quando utilizados elementos de contorno lineares e apenas uma sub região. O problema, reconhecidamente difícil para o MEC, foi satisfatoriamente solucionado quando aplicouse elementos de contorno quadráticos.

Na segunda parte deste trabalho, a formulação desenvolvida apresentou uma melhoria na análise de estruturas formadas pela associação de placas. A equação integral de contorno para a rotação (4.44), foi incluída na parte correspondente à elasticidade plana da formulação acoplada. Dessa maneira, as condições de compatibilidade de ro- 
tações puderam ser plenamente satisfeitas entre placas acopladas em qualquer direção. Para os problemas em que a rotação em torno do eixo $x_{3}$ não ocorria, os resultados obtidos na formulação apresentada e na convencional (sem a rotação $\omega_{3}$ ) não mostraram diferenças significativas. Mostrando que a inclusão do grau de liberdade não afeta a formulação original. Para o caso em que a rotação é imposta através de carregamentos laterais, os resultados mostraram um melhor acordo com aqueles obtidos usando o MEF.

A formulação desenvolvida se mostrou bastante robusta, sem qualquer limitação quanto à geometria ou tipo de condições de contorno. Desta forma, o MEC pode ser considerado uma alternativa interessante para a análise da associação de placas no espaço, competitivo com o MEF que é o principal método numérico usado para análise estrutural.

\subsection{Trabalhos Futuros}

Neste trabalho a formulação base do MEC para a associação de placas foi estendida com a inclusão da rotação $\omega_{3}$. O cálculo de deslocamentos e forças de superfície generalizadas foi realizado e desta maneira restam como possibilidade de trabalhos futuros:

- Eliminação das restrições de elementos retilíneos, com um tratamento mais geral para as hiper singularidades presentes na formulação;

- A inclusão do cálculo de tensões para formulação recém desenvolvida;

- A avaliação da inclusão de um momento correspondente à rotação $\omega_{3}$;

- Aplicação de métodos rápidos com o intuito de tornar o programa apto a tratar problemas de larga escala;

- Inclusão da rotação $\omega_{3}$ na formulação de cascas. 


\section{Referências Bibliográficas}

[1] M.H. Sadd. Elasticity: Theory, Applications, and Numerics. Elsevier Science, 2009.

[2] C.M. Wang, J.N. Reddy, and K.H. Lee. Shear Deformable Beams and Plates. Elsevier, 2000.

[3] T. Dirgantara and M. H. Aliabadi. Boundary element analysis of assembled plate structures. Communications in Numerical Methods in Engineering, 17(10):749$760,2001$.

[4] Y. Liu. Fast Multipole Boundary Element Method: Theory and Applications in Engineering. Cambridge University Press, 2009.

[5] A. Milazzo, I. Benedetti, and M.H. Aliabadi. Hierarchical fast bem for anisotropic time-harmonic 3-d elastodynamics. Computers and Structures, 96-97:9 - 24, 2012.

[6] G. Kirchhoff. Uber das gleichgewicht und die bewegung einer elastischen scheibe. J. Reine Angew. Math., 40:51-88, 1850.

[7] E. Reissner. On bending of elastic plates. Quart. Applied Mathematics, 5:55-68, 1947.

[8] R. D. Mindlin. Influence of rotatory inertia and shear on flexural motions of isotropic elastic plates. J. Applied Mechanics, 18:31-38, 1951.

[9] J.N. Reddy and N.D. Phan. Stability and vibration of isotropic, orthotropic and laminated plates according to a higher-order shear deformation theory. Journal of Sound and Vibration, 98:157-170, 1985.

[10] T. Westphal Jr. and H. Andränd E. Schnack. Some fundamental solutions for the kirchhoff, reissner and mindlin plates and a unified BEM formulation. Engineering Analysis with Boundary Elements, 25(2):129 - 139, 2001.

[11] F. Vander Weën. Application of the boundary integral equation method to reissner's plate model. International Journal for Numerical Methods in Engineering, 18(1):1-10, 1982.

[12] M.H. Aliabadi. The Boundary Element Method, Applications in Solids and Structures. The Boundary Element Method. Wiley, 2002. 
[13] Y.F. Rashed. Boundary element formulations for thick plates. Topics in engineering. WIT Press, 2000.

[14] J. Useche and E.L. Albuquerque. Dynamic analysis of shear deformable plates using the dual reciprocity method. Engineering Analysis with Boundary Elements, 36(5):627-632, 2012.

[15] J. Useche, E.L. Albuquerque, and P. Sollero. Harmonic analysis of shear deformable orthotropic cracked plates using the boundary element method. Engineering Analysis with Boundary Elements, 36(11):1528-1535, 2012.

[16] Adriana dos Reis, Éder Lima Albuquerque, and Leandro Palermo Júnior. The boundary element method applied to orthotropic shear deformable plates. Engineering Analysis with Boundary Elements, 37(4):738-746, 2013.

[17] Adriana dos Reis, Éder Lima Albuquerque, Fernando Luiz Torsani, Leandro Palermo Jr., and Paulo Sollero. Computation of moments and stresses in laminated composite plates by the boundary element method. Engineering Analysis with Boundary Elements, 35(1):105-113, 2011.

[18] E.L. Albuquerque and M.H. Aliabadi. A boundary element analysis of symmetric laminated composite shallow shells. Computer Methods in Applied Mechanics and Engineering, 199:2663-2668, 2010.

[19] G. Beer, I. Smith, and C. Duenser. The Boundary Element Method with Programming For engineers and scientists. Springer-Verlag/Wien, 2008.

[20] C.A. Brebbia and J. Dominguez. Boundary Elements An Introductory Course. WIT Press, 1992.

[21] J. C. Lachat and J. O. Watson. Effective numerical treatment of boundary integral equations: A formulation for elastostatics. International Journal for Numerical Methods in Engineering, 10:991-1005, 1976.

[22] Masataka Tanaka, Kouji Yamagiwa, Kenichi Miyazaki, and Takahiro Ueda. Free vibration analysis of elastic plate structures by boundary element method. Engineering Analysis, 5(4):182 - 188, 1988.

[23] M. Tanaka, T. Matsumoto, and A. Shiozaki. Application of boundary-domain element method to the free vibration problem of plate structures. Computers and Structures, 66(6):725 - 735, 1998. 
[24] P.M. Baiz and M.H. Aliabadi. Local buckling of thin-walled structures by the boundary element method. Engineering Analysis with Boundary Elements, 33(3):302 - 313, 2009.

[25] H. Leung and P.M. Baiz. Partition of unity and drilling rotations in the boundary element method (BEM). International Journal of Solids and Structures, 50:379$395,2013$.

[26] C. Chinosi. Shell elements as a coupling of plate and drill elements. Computers and Structures, 57(5):893-902, 1995.

[27] D.J. Allman. A compatible triangular element including vertex rotations for plane elasticity analysis. Computers $\&$ Structures, 19:1-8, 1984.

[28] P. G. Bergan and C. A. Felippa. A triangular membrane element with rotational degrees of freedom. Computer methods in applied mechanics and engineering, 50:25-69, 1985.

[29] P. G. Bergan and M. K. Nygård. Finite elements with increased freedom in choosing shape functions. International Journal for Numerical Methods in Engineering, 20:643-664, 1984.

[30] R. D. Cook. On the allman triangle and a related quadrilateral element. Computer and Structures, 22:1065-1067, 1986.

[31] K. Wisniewski and H. Turska. Enhanced allman quadrilateral for finite drilling rotations. Comput. Methods Appl. Mech. Engrg., 195:6086-6109, 2006.

[32] H. Nguyen-Van, N. Mai-Duy, and T. Tran-Cong. An improved quadrilateral flat element with drilling degrees of freedom for shell structural analysis. Computer Modelling in Engineering and Sciences, 49:81-110, 2009.

[33] M. Huang, Z. Zhao, and C. Shen. An effective planar triangular element with drilling rotation. Finite Elements in Analysis and Design, 46:1031-1036, 2010.

[34] R. Tian and G. Yagawa. Allman's triangle, rotational DOF and partition of unity. International Journal for Numerical Methods in Engineering, 69:837-858, 2007.

[35] D. I. G. Costa, E. L. Albuquerque, and P. M. Baiz. Boundary element method applied for folded thick plates. In V. Mallardo and M. H. Aliabadi, editors, Advances in Boundary Element and Meshless Techniques XV, 2014. 
[36] C.M. Wang, G.T. Lim, J.N. Reddy, and K.H. Lee. Relationships between bending solutions of reissner and mindlin plate theories. Engineering Structures, 23(7):838 $-849,2001$.

[37] J. N. Reddy. Energy Principles and Variational Methods in Applied Mechanics. John Wiley \& Sons, INC., 2002.

[38] J.H. Kane. Boundary Elements Analysis in Engineering Continuum Mechanics. Prentice Hall, New Jersey, second edition edition, 1994.

[39] Jurgen Friedrich. A linear analytical boundary element method (BEM) for 2D homogeneous potential problems. Computers \& Geosciences, 28(5):679 - 692, 2002.

[40] PaweŁ JabŁoński. Integral and geometrical means in the analytical evaluation of the $\{B E M\}$ integrals for a 3d laplace equation. Engineering Analysis with Boundary Elements, 34(3):264 - 273, 2010.

[41] Jaan Kiusalaas. Numerical Methods in Engineering with MATLAB. Cambridge University Press.

[42] J. C. F. Telles. A self-adaptive co-ordinate transformation for efficient numerical evaluation of general boundary element integrals. International Journal for Numerical Methods in Engineering, 24(5):959-973, 1987.

[43] L.C. Wrobel. The Boundary Element Method, Applications in Thermo Fluids and Acoustics. The Boundary Element Method. Wiley, 2002.

[44] A. Portela, M. H. Aliabadi, and D. P. Rooke. The dual boundary element method: Effective implementation for crack problems. International Journal for Numerical Methods in Engineering, 33(6):1269-1287, 1992.

[45] R.J. Marczak and G.J. Creus. Direct evaluation of singular integrals in boundary element analysis of thick plates. Engineering Analysis with Boundary Elements, $26: 653-665,2002$.

[46] C. Di Pisa. Boundary Element Analysis of Multi-layered Panels and Structures. Phd, 2005.

[47] S. S. Hoefel, E. L. Albuquerque, and P. Sollero. Modeling thin walled 3D composite structures using the boundary element method. In A. P. Selvadurai, C. L. Tan, and M. H. Aliabadi, editors, Advances in Boundary Element and Meshless Techniques VI, 2005. 
[48] S. Timoshenko. Theory Of Elasticity 3E. Engineering societies monographs. McGraw-Hill, 1936.

[49] C. E. Augarde and A. J. Deeks. The use of timoshenko's exact solution for a cantilever beam in adaptive analysis. Finite Elements in Analysis and Design, 44:595-601, 2008.

[50] Y.J. Liu and Y.X. Li. Slow convergence of the BEM with constant elements in solving beam bending problems. Engineering Analysis with Boundary Elements, 39(0):1-4, 2014.

[51] ANSYS Mechanical APDL Element Reference.

[52] ANSYS Mechanical APDL Theory Reference. 